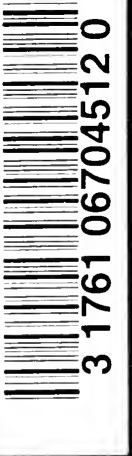

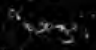







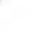




\section{Digitized by the Internet Archive in 2007 with funding from Microsoft Corporation}




$$
\text { is }
$$




\section{'THIS TRANSLATION IS DEDICATED}

- to the staff of the

BIRMINGHAM HOSPITAI FOR SICK CHILDREN

$$
\text { IN TOKEN OF THE }
$$

SENTIMENTS OF FRIENDSHIP

ENTERTAINED FOR THEM AND THEIR INSTITUTION

THE TRANSLATOR 



\section{AUTHOR'S PREFACE}

Fifteen years of uninterrupted activity in the Francis Joseph Hospital for Children in Prague, pursued partly under the guidance of my much esteemed teacher and patron, the Ministerialrath Freiherr Joseph von Löschner, and partly in the independent position of Teacher and Physician in Ordinary to the Hospital, have encouraged me and given me some claim to write this treatise. It has been my object to make it a trustworthy guide to the student as well as to the practitioner; but whether I have succeeded or not can only be determined by the public opinion to which I now submit the book, with the consciousness that $I$ have done my best.

Pragde; August, 1871. 



\section{TRANSLATOR'S PREFACE}

Dr. STEINeR's book has met with such marked success in Germany that a Second Edition has already appeared, a circumstance which has delayed the appearance of its English form, in order that I might be able to give his additions and corrections.

In Germany the use of the metric system has not yet entirely superseded the local measures; but it is rapidly doing so, as in England. I have, therefore, rendered all thermometric obserrations in the Centigrade scale, and all measurements in centiand millimètres.

I have added as an Appendix the "Rules for Management of Infants" which have been issued by the staff of the Birmingham Sick Children's Hospital, because I think that they have set an example by freely distributing these rules amongst the poor for which they cannot be sufficiently commended, and which it would be wise for other sick children's hospitals to follow.

I have also added a few notes, chiefly, of course, relating to the surgical ailments of children.

LAWSON TAIT.

Birminghasr, October, 1874. 



\section{CONTENTS}

\section{FIRST DIVISION}

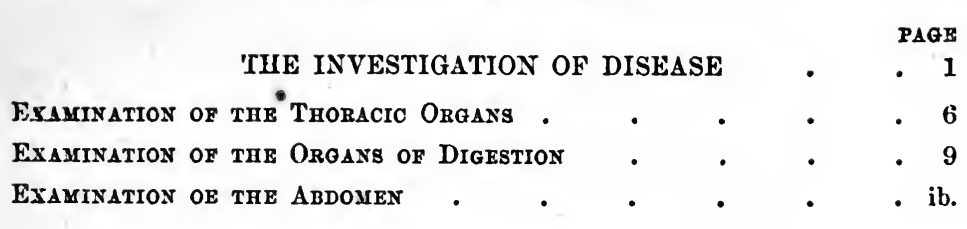

\section{SECOND DIVISION}

\section{DISEASES OF THE NERVOUS SYSTEM • • 14}

a. Diseages of tie Brain and its Membranes . . . . ib.

1. Anæmia of the brain and its membranes-hydrocephaloid . . ib.

2. Hyperæmia of the brain and meninges . . . 18

3. Hæmorrbages into the cranial cavity-meningeal and cerebral . 21

4. Thrombosis of the cerebral sinuses . $\quad$. $\quad . \quad 25$

5. Pachymeningitis-inflammation and hæmatoma of the dura mater . 28

6. Inflammation of the pia mater-meningitis simplex; lepto-meningitis 30

7. Meningitis tuberculosa; basal meningitis; tubercular inflammation

- of the membranes of the brain . . . . . 34

8. Encephalitis; inflammation of the brain $\quad . \quad$. $\quad . \quad$. 39

9. Hypertrophy and sclerosis of the brain $\quad$. $\quad$. $\quad$. $\quad .42$

10. Hydrocephalus, serous exudation in the brain and its membranes . 44

a. Congenital bydrocephalus . . . . . . ib.

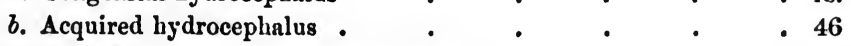

11. Tumours of the brain and its membranes $\quad$. $\quad . \quad$. 51

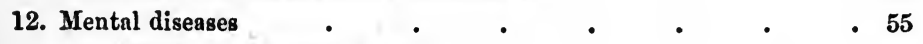

1. Mental excitement (mania) . $\quad$ - $\quad$. $\quad 56$

2. Mental depression (melancholia) . . . . . ib.

3. Mental weakness (imbecility) . $\quad$. $\quad$ • 57

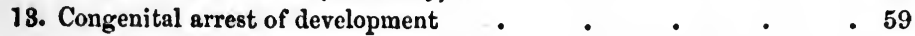

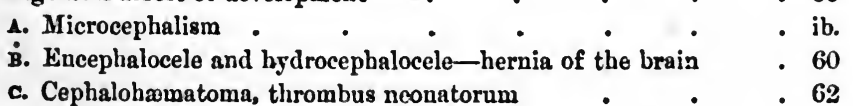


Diseases of the Spinat Cord and its Membranes •

1. Hyperæmia of the spinal marrow and its membranes. . . ib.

2. Meningitis spinalis-inflammation of the membranes of the spinal cord 65

3. Meningitis cerebrospinalis epidemica-cerebro-spinal meningitis . 67

4. New formations in the spinal cord and its membranes . 69

5. Spina bifida, hydro-rhachitis, hydromeningocele, hydromyelocele $\quad 70$

6. Sensoro-neurosis and hyperæsthesia . . . . 72

7. Neuroses of motor power $\quad$ - $\quad$ • $\quad$. 75

a. Eclampsia, convulsions, fits $\quad$ - $\quad . \quad$. $\quad . \quad$. ib.

Indirect, symptomatic, or reflex convulsions . . . 76

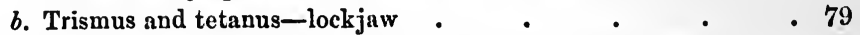

c. Spasmus nutans; nodding palsy; salaam convulsion of infancy . 82

d. Chorea minor; chorea Sti. Viti; ballismus; St. Vitus' dance;

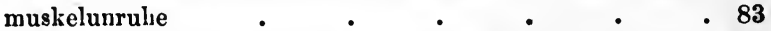

e. Chorea magua-German chorea (grosser Veitstanz) . 88

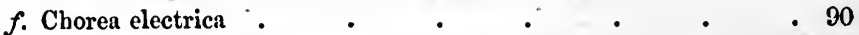

g. Epilepsy-falling sickness _ . . . . . 91

h. Akinesia-motor-paralysis-palsy $\quad$. $\quad$. $\quad$. 94

1. Essential paralysis in children-spinal palsy . . ib.

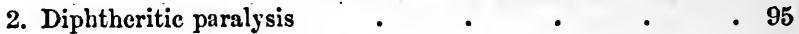

3. Paralysis of traumatic origin $\quad$ - $\quad$. $\quad$ • $\quad 96$

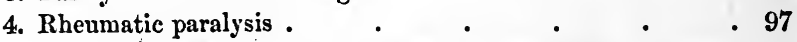

5. Paralysis from material changes in the central nervous system and from disease of bone . . . . ib.

6. Paralysis myo-sclerosica, the pseudo-hypertrophic muscular paralysis of Duchenne . $\quad$. $\quad$ • $\quad$. $\quad .98$

i. Arthrogryposis ; contractura artium; essential contractions .101

Neurotic atrophy of the face $\quad$. $\quad . \quad$. 102

\section{THIRD DIVISION}

\section{DISEASES OF THE ORGANS OF RESPIRATION 104}

\section{AsphyXia NeONATORUM}

a. Diseases of the Nasal Cavities

1. Catarrh; coryza ; cold in the head . • . . . ib.

2. Epistaxis; blenorrhagia ; bleeding at the nose . $\quad . \quad 107$

3. New formations in the nose and abscess of the septum . 108

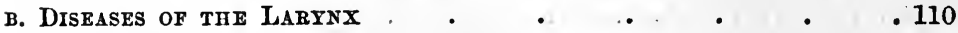

1. Laryngeal catarrh and pseudo croup . . . . . . . . ib.

2. Croup, laryngitis crouposa vel inaligna, exudative croup $\quad . \quad .113$

3. Tumours of the larynx $\quad . \quad \ldots \quad$. $\quad . \quad .125$

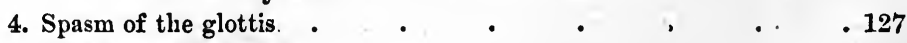

5. Paralysis of the glottis _ $\quad . \quad \ldots \quad \ldots \quad . \quad .130$

6. Foreign bodies in the air-passages $\quad . \quad+\quad . \quad . \quad .132$

c. Diseases of the Trachea $\quad$. $\quad$. . . . . . 133 


\section{Diseases of the Thyroid Body}

Goitre .

E. Diseases of the Lungs and Bronchi .

1. Bronchial catarrh, acute and chronic; dry bronchial catarrh .

2. Hooping-cough ; tussis convulsiva; pertussis .

3. Pneumonia-inflammation of the lungs

a. Catarrhal pneumonia-broncho-pneumonia-lobular pneumonia - ib.

b. Croupous pneumonia

4. Pulmonary empliysema

5. Atelectasis pulmonum .

6. Phthisis pulmonalis-consumption

Tubercular phthisis

Phthisis from chronic pneumonia and bronchitis

7. Gangrene of the lungs :

1. Pleuritis .

\section{FOURTH ḊIVISION}

\section{DISEASES OF THE ORGANS OF THE CIRCUIATION AND} OF THE LYMPHATIC SYSTEM

1. Patency of the foramen ovale

2. Putency of the ductus Botalli

3. Communication between the ventricles

4. Congenital stenosis of the pulmonary artery

5. Congenital tricuspid stenosis

6. Congenital aortic stenosis .

7. Pericarditis

8. Dropsy of the pericardium-hydropericardium

9. Endocarditis and valvular disease .

10. Vascular tumours, angioma, teleangicctasis

11. Inflammation of lymphatic glands, lymphadenitis

12. Anæmia lymphatica, pseudo-lencocythemia, adenia

\section{FIF'TH DIVISION}

\section{DISEASES OF THE ORGANS OF DIGESTION . 193}

Preliminary Observations on the Nodrishuent of Childrex - ib.

a. Modth AND Throat . $\quad$. $\quad$. $\quad$. $\quad . \quad . \quad .200$

1. Narrowing of the orifice of the mouth, microstomia . . ib.

2. Harelip and cleft palate . $\quad . \quad$. $\quad .201$

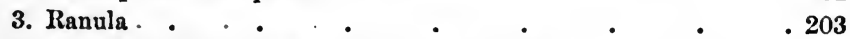

4. Anchylo-glottis; tongue-tie or tongue-tack . . . ib.

5. Catarrh of the mouth; stomatitis catarrhalis, S. simplex, S. ery thematosa.

6. Aphtha; stomatitis aphthosa 
PAGE

7. Croupous inflammation of the mucous membrane of the mouth; stomatitis et angina crouposa

8. Diphtheria ; angina diphtheritica $. \quad . \quad . \quad$ ib.

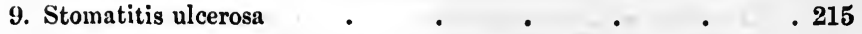

10. Gangrene of the mouth; noma . $\quad . \quad . \quad . \quad 216$

11. Parasitic aphtha; stomatomykosis (Mehlmund) . . . $\quad 219$

12. Dentition and its dangers $\quad . \quad \ldots \quad$. $\quad . \quad 221$

13. Tonsillitis, angina tonsillarum . $\quad . \quad \ldots \quad . \quad 224$

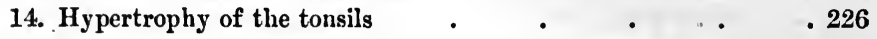

15. Retro-pharyngeal abscess. $. \quad . \quad . \quad . \quad .227$

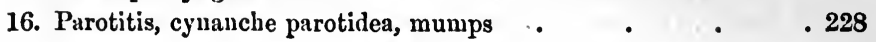

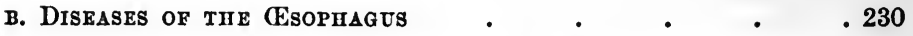

c. Diseases of the Stomach and Intestines - . $\quad . \quad .233$

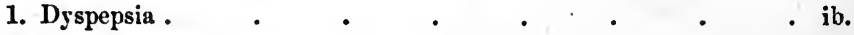

2. Gastric catarrh . $\quad . \quad$. $\quad .234$

3. Croupo-diphtheritic inflammation of the stomach; gastritis croupo-diphtheritica . $\quad . \quad \ldots \quad . \quad . \quad 236$

4. Round perforating ulcer of the stomach . $\quad .{ }^{\circ} \quad .237$

5. Hæmorrhagic erosions of the gastric mucous membrane . . ib.

6. Follicular and tubercular ulceration of the stomach $\quad . \quad .238$

7. Gastromalacia; softening of the stomach . . . ib.

8. Intestinal catarrh . . . . . . . ib.

a. Acute gastric catarrh; gastro-enteritis cholcriformis ; cholera nostras; cholera infantum $\quad . \quad \ldots \quad . \quad .239$

b. Acute intestinal catarrh • . . . . . 241

c. Chronic intestinal catarrh and follicular ulceration; catarrhus - intestinalis chronicus; enteritis follicularis, tabes meseraica $\quad \therefore \quad . \quad . \quad . \quad . \quad . \quad .243$

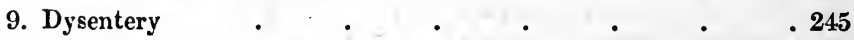

10. Constipation, intestinal obstruction, koprostasis . $\quad . \quad .247$

11. Stricture and occlusion of the intestinal canal . $\quad . \quad 249$

12. Invagination, or intussusception . . . . . . ib.

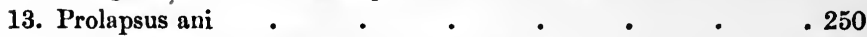

14. Polypus of the rectum $\quad . \quad \ldots \quad \ldots \quad$. $\quad . \quad .251$

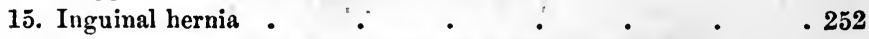

16. Animal parasites-worms, helminthiasis . $\quad . \quad \therefore \quad .253$

$a$. The round worm, ascaris lumbricoides . $\quad . \quad$ ib.

b. Threadworms; oxyuris vermicularis $\quad . \quad .255$

c. Tapeworm; tænia . . . . . . 256

d. Trichocephalus dispar $\quad . \quad$. $\quad . \quad .257$

D. Diseases of the Peritonedar • . . . . . . . ib.

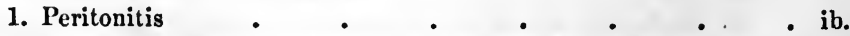

2. Tubercular peritonitis and tubercle of the peritoneum . $\quad .261$

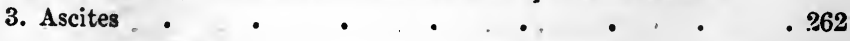




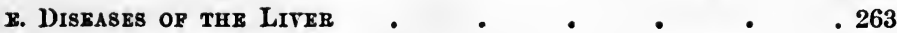

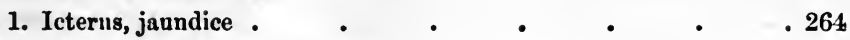

2. Fatty infiltration of the liver $\quad$ - $\quad$ - $\quad . \quad 266$

3. Futty degeneration and atrophy $\quad . \quad$ - $\quad . \quad 4 \quad .267$

4. Lardaceous, waxy, or amyloid degeneration of the liver . . ib.

5. Syphilitic inflammation of the liver $\quad . \quad$. $\quad . \quad 268$

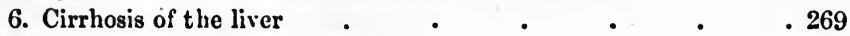

F. Diskases of the Spleex $\quad . \quad$. $\quad . \quad$. $\quad . \quad 270$

1. Acute enlargement $\quad$. $\quad$ - . . . . ib.

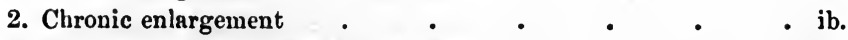

3. Splenitis, inflammation of the spleen $\quad$. $\quad . \quad$. $\quad .271$

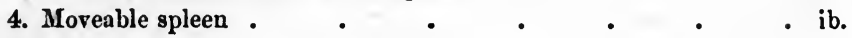

\section{- SIXTH DIVISION}

DISEASES OF THE URINARY AND SEXUAL ORGANS $\mathbf{2 7 2}$

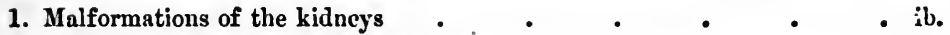

2. Hyperæmia and anæmia of the kidneys . $\quad . \quad+\quad . \quad . \quad$ ib.

3. Hæmorrhage from the kidneys and hæmaturia . $\quad . \quad . \quad 273$

4. Inflammation of the parenchyma of the kidneys . . . . ib.

Nephritis albuminosa $\quad . \quad$ - $\quad . \quad$ e $\quad . \quad$. ib.

Anatomy of Bright's disease (in its more restricted sense) . . ib.

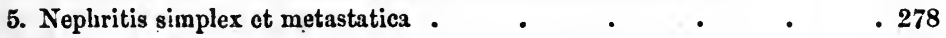

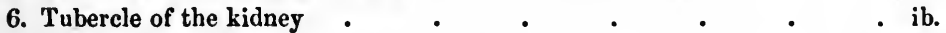

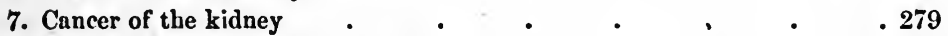

8. Cystic kidney; hydronephrosis . $\quad . \quad$. $\quad . \quad 280$

9. Concretions in the kidney. $\quad . \quad$. $\quad$. $\quad$. $\quad . \quad 281$

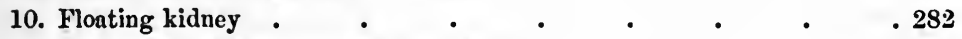

11. Congenital malformations of the bladder . $\quad$ - . $\quad$. 283

12. Catarrh of the bladder; cystitis . . . . . . . ib.

13. Tubercle of the bladder . $\quad$. $\quad . \quad$. 285

14. Stone in the bladder $\quad . \quad$. $\quad . \quad$. $\quad . \quad \ldots \quad$. ib.

15. Enuresis, incontinence of urine, bed-wetting $\quad$ - $\quad$ • $\quad$. $\quad 288$

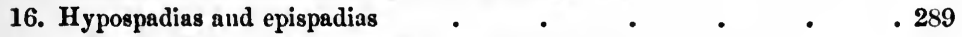

17. Cellular adhesion of the prepuce to the foreskin . . . . ib.

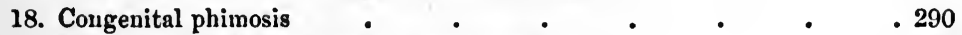

19. Inflammation of the foreskin; balanoposthitis . $\quad . \quad . \quad 291$

20. Paraphimosis . . . . . . . . ib.

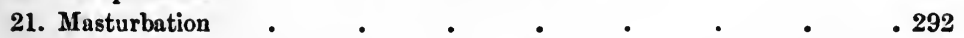

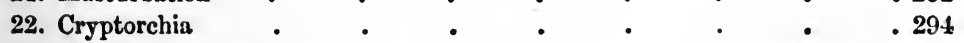

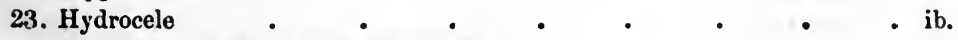

a. Hydrocele of the tunica raginalis . . . . . ib.

b. Hydrocele of the cord . $\quad . \quad \ldots \quad$. $\quad . \quad \ldots \quad .295$

24. Cellular atresia of the vulia $\quad$. $\quad . \quad$. 296 
25. Vulvo-vaginitis

a. Catarrh of the genital inucous membrane; vulvo-vaginitis catarrhalis;

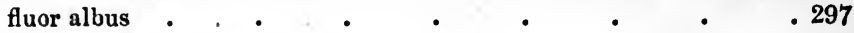

b. Vulvo-vaginitis phlegmonosa . . . . . . ib.

c. Vulvo-vaginitis diphtheritica $\quad . \quad \ldots \quad$. $\quad . \quad .298$

d. Vulvo-vaginitis gangrenosa $\quad$. $\quad . \quad 0 \quad$. $\quad . \quad .299$

26. Swelling and inflammation of the mamma ; mastitis . . . . ib.

APPENDIX $. \quad . \quad .300$

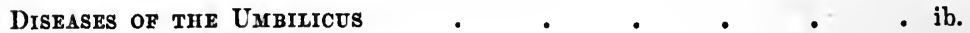

1. Fissura abdominalis . $\quad . \quad$. $\quad . \quad$. $\quad .301$

2. Hernia funiculi umbilicalis . $\quad$. $\quad$ - . . . . ih.

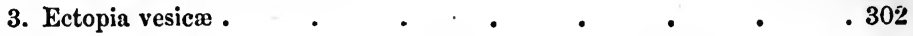

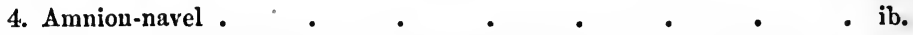

5. Sarcomphalus, fungus umbilici, raspberry-navel . . . ib.

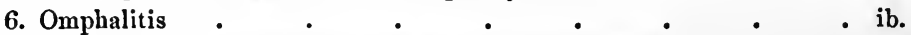

7. Thrombosis and inflammation of the umbilical vessels . . 303

8. Gangrene of the umbilicus $\quad . \quad 5 \quad \ldots \quad . \quad . \quad 304$

9. Omphalorrhagia, hæmorrhage from the umbilicus . . . ib.

10. Umbilical hernia $\quad$. $\quad . \quad$. $\quad . \quad$. 305

\section{SEVENTH DIVISION}

GENERAL DISEASES OF NUTRITION • • 306

1. Rachitis; Rickets; Englische Krankheit; Osteomalacia . . ib.

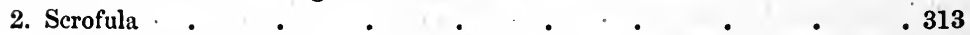

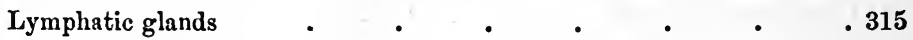

Skil and subcutaneous cellular tissue . $\quad$. $\quad . \quad$. $\quad . \quad 316$

Mucous membranes and organs of sense $\quad . \quad \ldots \quad$. $\quad .317$

Bones . . . . . . . . . . . . . 318

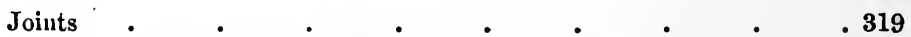

General course and result of scrofulosis $\quad$. $\quad . \quad .320$

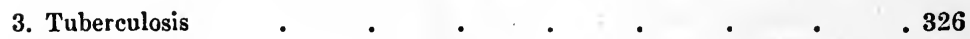

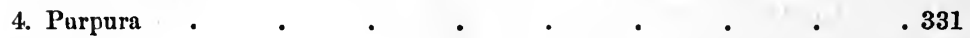

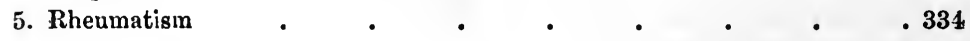

\section{EIGHTH DIVISION}

1. Scarlet fever

2. Measles, morbilli

3. Rötheln, rubeola

4. Variola, smallpox .

5. Varicella, chicken-pox

6. Vaccina and vaccination 
PAGE

7. Typhoid fever, typhus abdominalis, ileotyphus $\quad$. $\quad$ • 360

8. Relapsing fever, febris recurrens . $\quad$ - $\quad$ • $\quad$ - 366

9. Intermittent fever; ague . $\quad . \quad \ldots \quad$. $\quad . \quad$. 368

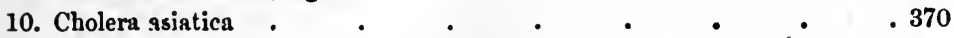

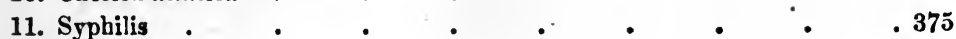

\section{NIN'TH DIVISION}

\section{DISEASES OF THE SKIN . . . 380}

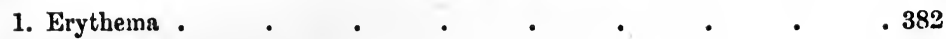

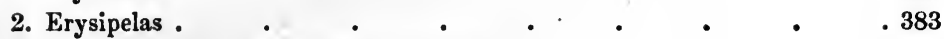

3. Dermatitis folliculosa (Acne cachecticorum) . $\quad$. $\quad$. $\quad .385$

4. Urticaria, nettle-rash $\quad . \quad+\quad . \quad+\quad . \quad$. $\quad . \quad$. ib.

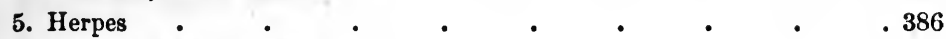

6. Pemphigus and pompholyx $\quad . \quad$. $\quad . \quad$. $\quad . \quad .388$

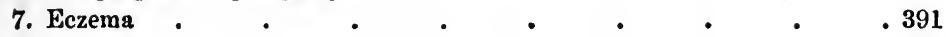

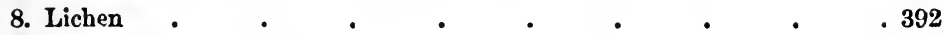

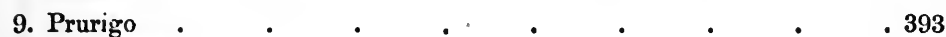

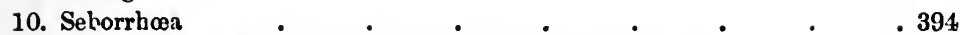

11. Alopecia areata, Area Celsi, Porrigo decalvans (Willan) . . . . ib.

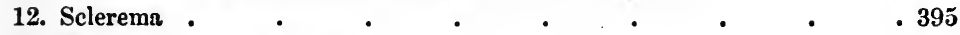

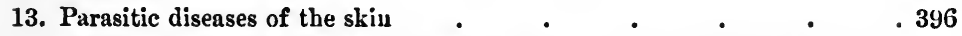

Herpes tonsurans, Herpes circinatus (Bateman), Trichomykosis, ringworm, Trichophyton tonsurans . . . . . ib.

Favas, tinea vera, porrigo favosa . $\quad . \quad$. $\quad . \quad$. 397

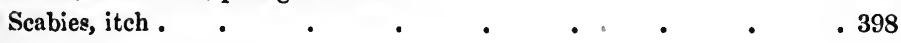

\section{APPENDIX}

RULES FOR THF MANAGEMENT OF INFANTS . . 401

(Issued by the Staff of the Birmingham Hospital for Sick Children.)

1. Warmth, cleanliness, fresh air $\quad$. . . . . . ib.

2. Nourishment while the child is under seven months old . . . ib.

3. How to bring up "by hand" . . . . . . ib.

4. Importance of regular feeding $\quad . \cdot \quad . \quad . \quad . \quad . \quad . \quad 402$

5. Nourishment when the child is over seven months old . . . . 403

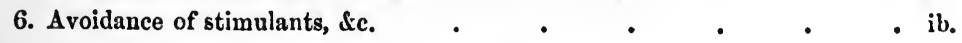





\section{THE}

\section{DISEASES OF CHILDREN}

\section{FIRST DIVISION}

\section{THE INVESTIGATION OF DISEASE}

The examination of sick children is associated with manifold difficulties and impediments, which the practitioner must learn to overcome if he wishes, as surely he must, to arrive at a safe diagnosis. The absence of speech, the uncertainty of the communications concerning the subjective disturbances of the condition of health, the wilfulness, dislikes, fear and agitation of the child, make an inquiry difficult or even impossible, and this so much the more if the practitioner does not understand how to win for himself the confidence of the patient. Let the child's rnctor be first the child's friend, and in his early visits especially let him not put his medical function in too great prominence, but, first of all, distract attention from himself to some other object, such as a toy, for thus he will the better gain his purpose.

Such precautions are especially advisable in children between six months and three years old, for in sucklings and in children over three years the difficulties are not so great, the former having no will and the latter being old enough to be accessible to rational impressions.

In making an examination $I$ am in the habit of so proceeding that I first of all obtain a gencral impression of the child examined, and then consider, seriatim, the various organs, unless the urgency of the case should be such as to demand immediate inquiry into 'the seat of the disease. 
For this purpose the child should be undressed, and the crib so placed that light should fall conveniently upon it, or let him be brought in arms near to a window or light. The general appearance is the sum total of the physical and mental conditions and actions, and a knowledge of this the experienced practitioner may gain without touching or disturbing the child; and from this alone he may, in many cases, be able to establish a probable or even an accurate diagnosis.

First of all, in this general inspection is to be noticed the degree of development and condition of the child as regards nourishment; and for this purpose it should be borne in mind the newly born male child measures, on the average, forty-nine centimètres and the female about forty-eight.

The child grows quickest in the first week of its life; in the first year it increases by nearly $16-20$ centimetres; in the second, about $8-10$; in the third, about $7-8$; in the fourth, about 6 ; from the fifth until the fifteenth or sixteenth year the yearly growth amounts to about $5-6$ centimètres. The weight of the body of a new-born child averages from three to four thousand grammes.

The daily increase of weight of a normally developing infant amounts to from a quarter of an ounce to three quarters of an ounce.

A comparison of the actual state of the child with its age wiil tell at a glance whether its development and nourishment be normal or not, by revealing deficiencies in the length of the body and in the fat-cushions, in the firmness of the muscles, and fulness and smoothness of the skin. Such deficiencies have their origin either in deficient nutrition or in actual disease. The relations of the parts and organs to one another must also be noticed on inspection, and beginners must remember that in the new-born and in sucklings the head and abdomen are larger, in proportion to the rest of the body, than they are in after life. Striking alterations of these relations may indicate hydrocephalus, microcephalism, rickets, chronic intestinal catarrh, or caries of the vertebral column, \&c.

The attitudes and movements of the child must also be carefully noticed during the general inspection. Healthy children lie quiet, and move themselves in the waking condition with crident enjoyment and in vigorous and lively ways; apathetic 
indifferent decubitus indicates loss of power and weakness, or the child may be unconscious or semiconscious, the eyes staring and not moving, the eyelids half open, indicating thereby signs of a severe affection of the brain. Unquiet twitchings and turnings, together with frequent changes of position, arise from febrile illness and often precede brain affections. Frequent extension and retraction of the lower extremities upon the abdomen, associated with painful cries, indicate, in sucklings, dyspepsia and colic, and continued retraction of both the lower extremities accompanies peritonitis; but if limited to one limb, then gonitis, coxitis, or psoitis may be suspected.

When there is evident anxiety on the part of the child to retain one position, as on the back, or on one or other side, together with shart, quick respirations, some inflammatory change may be suspected in the respiratory or abdominal organs. If the head be strongly bent backwards there may be exudative mischief in the brain, or croup. In affections of the brain the child indicates its suffering by frequent restless movements of its head on the pillow, by purposeless movements of one or both upper extremities. Photophobia is evidenced by the child persistently lying on its face, and is attendant on scrofulous inflammations of the eyes. When the child is seen in a half sitting position, with more or less marked difficulty of breathing, pleuritic and pericardial effusions must be looked for. Central or peripheral lesions of the nervous system are indicated by clonic and tonic contractions, or paralysis of isolated muscles or of muscular groups, and chorea is shown by its peculiar irregular movements, with persistence of consciousness.

The expression of the face is an important feature of the inspection of the child. In healthy infants, during the first few weeks of life, there is no special physiognomy, but under the influence of certain diseases the expression of the face becomes altered in very important and characteristic ways. A pinched and wasted face accompanies chronic diseases, especially of the intestinal canal and lungs. When a collapsed appearance of the face, with sinking of the eyes, has appeared in a few hours or days, some very serious affection has certainly supervened, as acute abdominal disease, cholera infantum, suppurative peritonitis, or diphtheritic gastritis. A look of dis- 
tress in the eyes of the child, wrinkling of the skin of the forehead, are seen in brain affections; exaggerated movements of the nasal cartilages almost always may be noticed in acute affections of the chest; and œdema of the eyelids is usual in dropsy from nephritis, and also from the straining of hoopingcough.

After having completed the general inspection, and during the time thereby occupied having ascertained the facts of the duration and previous details of the illness, the special inrestigations of the temperature, the pulse, and respiration frequency, may then be made, but it must be remembered that then these conditions may vary very greatly in the child from very slight causes. If the child be asleep the observations may be made without difficulty, but it may be very different if the child be restless and crying, and nothing can be done until it is quietened.

The average number of the pulse frequency in the different periods of infantile age may be gathered from the following figures (Rilliet and Barthez, Valleix, \&c.), which make the variations more intelligible:

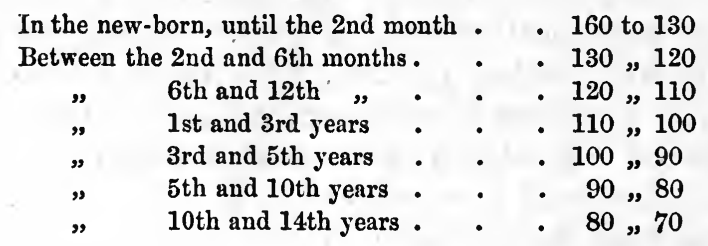

In the review of a feverish condition in the child the indication of the increased temperature is of more importance than increased pulse frequency. In general, the pulse curve in the child follows the course of the temperature. According to most observers the pulse curve in children under four years old, in non-febrile conditions, coincides with the temperature curve; in older children it falls under the latter; in feverish diseases in children under four years above the temperature curve; and similarly in older children. Almost more important than the frequency is the quality and rhythm of the pulse. A somewhat irregular and depressed pulse is met with in brain disease, heart disease, and sometimes, also, in nervous, anæmic children, without 
any important significance ; simple retardation, with irregularity of pulse, accompanies the sclerema of the new-born, often, also, parenchymatous nephritis and jaundice. A pulse frequency of $140-160$, with an abnormal elevation of temperature in the morning hours, may caution us to anticipate a pneumonia or the outbreak of an acute exanthem. The relative temperature of the body must be noticed, for it is important and necessary for a correct estimation of the course of the fever.

A rough estimate of the degree of fever may be made by the application of the hand of the observer, and any difference in temperature of the various regions of the body ought to be noted in this way. Thus, one finds in tubercular meningitis the temperature of the head really increased, and that of the feet, on the other hand, depressed ; in typhus the forehead and abdomen exhibit usually the highest temperature; while in acute exanthemata the abnormal height of temperature is uniform over the whole surface of the skin. Accurate measurements of the blood temperature can be obtained by placing an indicating thermometer in the gut or in the axilla. In newborn children the temperature is from a quarter to half a degree Centigrade higher than in the adult, and during infancy it is even more than that. A rapid elevation of temperature in children of a very transient character is often met with, and may be due to a slight catarrh or an attack of dyspepsia. A rigor at the beginning of a feverish disease is met with rarely in children, the younger they are the more seldom, and when it is present, it is usually imperfectly marked. Instead of this, we often see an attack of convulsions; in older children delirium occurs, which is due to the ready excitability of their brains. Abnormal depression of temperature may be induced and continued by loss of blood and fluids in cholera morbus; in wasting, anæmic children; and further, from obstruction of the circulation, especially in heart disease, sclerema of the new-born, emphysema, asphyxia, collapse, and in the agony.

While examining the skin for its temperature, notice should also be made of its other conditions, whether dry or moist, or whether an eruption is present, and, if so, of what character.

In the examination of the head there is, first of all, to be determined its size and shape. In hydrocephalus, rickets, and in hypertrophy of the brain, it is abnormally large, and 
abnormally small in congenital or premature synostosis, producing microcephalism. In hydrocephalus its shape is roundish or oval, while in rickets it is wedge-shaped and marked ly uneven prominences on the forehead and on the parietal bones. The condition of the sutures and fontanelles is also of importance. The former gradually lose their motility during the second month of life. The posterior fontanelle is closed first, then the small lateral, and finally the great anterior fontanelle, between the fifteenth and twentieth months. Under abnormal circumstances the sutures and fontanelles may remain an unusually long time open and movable. Intercranial hyperæmia, exudation, effusion of blood or serum, may arch the sutures and fontanelles tightly outwards, while in anæmia and atrophy they have a tendency to sink inwards, and the edges of the bones glide over one another, forming terrace-like projections. Before the closure of the great fontanelle two motions are perceptible in it, one due to the cerebral pulsations, and another influenced by expiration and inspiration. On auscultation of the fontanelle the respiratory murmur and râles of deglutition may be heard, and also generally a more or less loud systolic bruit, the presence or absence of which seems to be of little diagnostic importance. A new aid in diagnosis of cerebral affections has been supplied in the ophthalmoscope, which frequently discloses appearances of great importance, as in cases of acute tuberculosis or in capillary embolism of the ophthalmic artery.

\section{Examination of the Thoracic Organs}

In children this is generally more difficult, and its results less positive and trustworthy than in the adult. The smallness of the organs, the delicacy of the tissues, the restlessness of the child, the absence of speech, together with the superficial and sparse breathing, contribute to this. The number and character of the respirations is first to be noticed, and these vary with the age, \&c., of the child. According to most observers, suckling infants in health make from 24 to 30 respirations in the minute, but they are not always rhythmic, either during sleep or when the child is awake. Later in life the respirations are generally a fourth or a fifth of the pulse beats. The more the 
breathing area of the lungs is lessened by disease the higher the number of respirations, which may reach to as many as 60 to 80 in the minute. Abnormally accelerated respiration occurs in children during the course of diseases characterised by severe feverish symptoms, such as the acute exanthemata, acute inflammatory affections of the thoracic viscera, and in diseases of the bones, such as rickets. The respirations may be unusually retarded in certain affections of the brain, and may fall as low as 16,12 , or even 8 in the minute. In such cases the breathing is generally irregular, sometimes being scarcely audible, and at other times being deep and loud. In newborn children and sucklings the respiration is chiefly abdominal, but later it is conducted both by the abdominal and thoracic muscles, and of the latter chiefly those of the anterior wall. The presence of peripneumonial furrows (Furche), due to the violent indrawing of the diaphragmatic insertions during inspiration, is always indicative of some hindrance to respiration, which may exist in the lungs (pneumonia), or in the air-passages (croup or bronchitis), or in the bones or muscles of the chest (rickets).

Physical examination of the chest in children is always best conducted without the aid of stethoscopes and pleximeters, and in a number of cases it is not enough to examine only the posterior surface when we have to examine an affection of the lungs. Children when swaddled are best examined when laid on their backs or sides, or if they can be carried erect, let them rest on the arm of the mother or nurse. For percussion, the examiner ought to wait till the child is quiet, for the note may be so altered by the screaming and struggling of the child as to be mistaken for a pathological indication, especially on the right side, and similar alteration may be produced by the position of the child. The percussion must be light and gentle, as otherwise it will produce pain to the child, and will probably rouse it, thus making any further examination difficult or even impossible. Auscultation is best immediate, save in disease of the heart, when the stethoscope becomes necessary. Regarding the pulmonary sounds, it is important to remember that the vesicular nuurmur is so much more intense than it is in later life that, by the inexperienced practitioner, it may be misinterpreted as a morbid sign; further, that the bronchial breath- 
ing of consolidation in feeble young children is often communicated over the healthy side.

Palpation is never to be omitted, as by it the condition of the vocal fremitus is to be determined, and the presence or absence of loud bronchial râles.

There still remain two signs - the cough and cry of the child-to which the practitioner must pay attentive heed. The former, when of simple catarrhal origin, is loose, but when the bronchial secretion is scanty it is shrill and piping. In inflammatory affections it is short, dry, interrupted, and evidently painful. A peculiar muffled, hoarse, and barking cough is met with in laryngeal catarrh and in croup. Hooping-cough has its peculiar cough, characterised by fitful and long-drawn inspirations, followed by violent spasmodic coughing, during which the child would seem in danger of choking. Chronic broncho-adenitis has a peculiar dry, provoking cough, making its appearance generally at night, and in tubercular children a dry, hacking cough continues day and night. In delicate and anæmic nervous girls, near the accession of puberty, a dry, painful, most obstinate and tormenting cough is sometimes met with, having the peculiarity of disappearing entirely at night.

Expectoration is suppressed in children until about six or seven years of age, with the single exception of hooping-cough, when it is brought up by the vomiting.

The cry of children is often a useful aid to diagnosis, indicating by its tone, frequency, and duration many various conditions of the patient. The frightened, wilful, or pampered child gives vent to a loud, long-drawn-out cry, declining as the expiration comes near an end, and momentarily interrupted during inspiration. Such cries completely exclude acute thoracic affections, but are frequently caused by abdominal pain, as the dyspeptic colic of sucklings. A smothered cry, without resonance, is met with in exudative laryngitis, and in laryngeal catarrh one somewhat similar, though less marked, is found. The child suffering from hydrocephalus gives vent to a series of disconnected, short, sharp cries. Painful cries, whimpering, and moaning characterise nearly all inflammatory and exhausting diseases. 


\section{Examination of the Organs of Digestion}

This forms an important feature in the diagnosis of diseases of children, and includes inspection of the cavity of the mouth and throat, the palpation and percussion of the abdomen, the inspection of the vomit and alvine evacuations. The examination of the mouth and throat ought never to be omitted, though there may seem no great occasion for it, and though the goodwill of the patient is often sacrificed for a long time by its performance. In children at the breast it is generally sufficient to depress the chin and lower lip, and passing the finger backwards to depress the tongue, in order to obtain a glimpse of the throat, and if the child cries during the process it will be much faciiitated. With older children it is much more difficult, as they are often very unwilling to open the mouth, and frequently force is necessary to make a satisfactory examination. The mouth may be opened by holding the nose, and then, by an expert movement with the handle of a spoon, the tongue may be depressed and a good view obtained, but if this manœuvre has to be employed the hands and feet of the child must be well secured.

In the examination of the mouth and throat the appearance of the tongue must be noted, and any indication of the beginning of scarlet fever, diphtheria, smallpox, \&c., must be looked for, together with any malformation or irregularity of the structures. When scarlet fever or diphtheria are known to be prevalent, the examination of the throat ought never to he omitted, no matter what symptoms are present.

\section{Examination of the Abdomen}

This is often difficult, or even impossible, from the straining and struggling of the child, but by placing it in the lap of the nurse, and engaging its attention by some toy, it may be sufficiently quietened to allow the hand of the observer to pass over the abdomen. Inspection of the abdomen will reveal any disproportion of its size or form, the condition of the abdominal integuments, and, what is of great consequence, of the umbilicus. Palpation will give us a rough estimate of the temperit- 
ture, while the tension or pliability of the integuments, the presence or absence of pain on pressure, or of glandular or other enlargements, may be ascertained by this means of examination. Percussion is best made by the middle finger of the right hand, tapping gently on the corresponding finger of the left will indicate whether any distension is due to flatulence or to fluid or solid accumulation. By it, also, organs may be mapped out and their relative sizes determined.

Distension of the abdomen is generally uniform, but it may be partial and circumscribed. It is most frequently due to distension of the intestines by gas, and this may accumulate to a serious extent in dyspepsia, intestinal catarrh, especially when chronic, in typhoid fever, and in tuberculosis of the mesenteric glands. Irum-like distension of the abdomen, with great tenderness, and muffled tympanitic note, is indicative of general peritonitis. In ascites the distension is more barrelshaped, and the abdomen is not painful to the touch. Percus. sion also indicates the presence of fluid by the tympanitic note, moving always to the upper part of the abdomen, in reference to the position of the patient, and the wave of fluctuation will be felt across all the diameters of the abdomen.

Distension of the epigastritum indicates gaseous distension of the stomach or transverse colon. When situated in the midabdominal regions, especially round the umbilicus, there may be tubercular disease of the mesenteric glands or an abscess. Fulness in the left hypogastric regions is met with in tumours of the spleen and left kidney, and these are usually painless. Swelling, accompanied by great tenderness, is found in the right iliac region when perityphlitis is present, and almost always, to a greater or less extent, in typhoid fever. Psoas abscess also may point in this region, and is found more frequently on the right side than on the left, always with marked disinclination to move the limb of the affected side.

In fulness of the hypogastric region care must be taken to ascertain that it is not distension of the bladder. Circumscribed peritonitis causes this appearance in exceptional cases.

A shrunken and collapsed condition of the abdomen is often met with in serious cases of disease of the brain, especially of tubercular meningitis, also in some intestinal affections, as cholera infantum, follicular enteritis, and dysentery. In the 
cerebral cases the abdominal integuments are often drawn in to such an extent as to present a scaphoid hollow (Kahnförmige Einziehung), and the peristaltic movements of the intestines may be evident through them.

There are two varieties of abdominal pain which deserve especial notice as being useful in diagnosis - the colicy, paroxysmal pain seen in cases of dyspepsia, diarrhœa, and flatulent distension, and the persistent ceaseless pain of inflammatory origin, which is increased on pressure. The former lasts only a few minutes, has longer or shorter remissions, and is not accompanied by symptoms of fever; while the latter has unmistakable concomitant pyrexial symptoms, and is well indicated by the evident distaste on the part of the child to all movement, the persistent lying on the back, and the increase of pain on the passage of flatulence or fæces.

Vomiting in infants is a very common phenomenon, and while in many cases it may be of little significance, in others it may be of the gravest import. For its proper interpretation its probable cause must be sought, the nature and method of its onset, the quantity and quality of the vomited matter, and the other symptoms accompanying the vomiting, must all be considered. It is probable that vomiting is easier in the child than in the adult, from the more vertical position of the stomach; and it may be due to improper nourishment, foreign substances in the stomach-as bile, lumbrici, or coins, \&c., or to textural diseases of the stomach itself. Further, it may arise sympathetically from diseases of such viscera as are supplied by the vagus, in some central irritation of the nervous system, from disease of the brain, in specially prolonged diseases of the fluids, and in alteration of the constitution of the blood, especially at the onset of the exanthemata.

What is often known as habitual romiting, also as the curdling of sucklings (Käsen der Säuglinge), strictly speáking, is not vomiting, but merely the emission of already used milk, and has no pathological signification. It occurs in many infants so readily that a short time or almost immediately after taking the breast, they will bring back some of the milk almost unaltered and without any discomfort or great effort. This kind of vomiting almost exclusively occurs in those children fed at the breast, and seldom in those artificially fed. A sudden onset 
of romiting, when the child has not been in the habit of it, with evacuation of a slimy white or yellowish-green fluid, is often a symptom of cerebral irritation, and occurs in tubercular meningitis, hydrocephalus, and in Bright's disease, \&c., being always a serious symptom.

The character of the matter vomited is always of importance, as to whether the food is undigested or mixed with foreign matter, as bile, blood, and exudation, \&c. Microscopic examination of the vomited matter will often be of service.

In examining the state of the evacuations, their quantity, frequency, and appearance are to be considered, whether also they are passed with or without pain, and at what time they are passed. No word of mouth description by the nurse should be trusted if the evacuation itself can be seen, and then there are to be noticed the consistence, colour, and the presence or absence of albuminous flakes, undigested food, blood, \&c. Flaky yellowish or yellowish-green evacuations, with whitish cheesy lumps intermixed, are characteristic of dyspepsia ; when there is deficiency of bile the stools are doughy, greyish or clay coloured; and when there is any breach of the mucous membrane, as in follicular enteritis, typhoid fever, dysentery, and tubercular disease of the intestines, they are mixed with blood or with altered clot and shreds of mucous membrane. In enteritis choleraformis the evacuations are almost free from offensive smell; in dyspepsia and the intestinal catarrh of sucklings they have a sourish smell; but in chronic ulcerative enteritis, and in intestinal tuberculosis, they have a penetrating carrion-like odour. The ova of intestinal worms may be detected by microscopic examination of the evacuations.

The examination will be incomplete without a special inspection of the umbilicus, anus, and the genitals, the two latter especially, for it is there that hereditary syphilis gives its first indications.

Further, in addition to the objective examination, there must be a careful inquiry into the history, which can be made only by questions directed to the parents and attendants. The family history must be inquired into, and the condition of the mother during her pregnancy. Indications of scrofula and tuberculosis must be looked for, though it will be found more expedient to do this indirectly than by directly naming those dreaded 
cachexias. If the patient is an infant, the practitioner must ask how it is fed, whether at the breast, by wet nurse, or by artificial food, and if by the latter, of what it consists. If the child is more advanced, the history of its dentition must be inquired into, and many other points.

Above all, listen carefully to the narration of the mother or nurse, and regard no fact as of slight importance. 


\section{CHAPTER II}

\section{DISEASES OF THE NERVOUS SYSTEM}

\section{A.-Diseases of the Brain and its Membranes}

\section{Ancmia of the Brain and its Membranes-Hydrocephaloid}

ANemia of the brain in infancy is relatively a frequent symptom, appearing under the influx of numerous acute and chronic diseases. Spanæmia in children produces comparatively more severe symptoms than in adults.

The intimate relation between anæmia and the interruption of the activity of the brain is still obscure, though there can be no doubt that the alteration of the fluid-contents of the vessels of the brain has very definite lesions for its results. From the resemblance of the symptoms which accompany it to those of acute hydrocephalus, Marshall Hall has given to it the name of hydrocephaloid, and this name has retained its semeiological significance.

Anatomy.-Anæmia of the brain is either general or circumscribed, the former being much the more common condition. 'The blood-vessels of the meninges are thin and collapsed, are occupied by a small quantity of pale blood, and in the subarachnoid cellular tissue is often found a quantity of clear serous fluid. The brain is generally small and soft, and slenderly marked, with the grey substance pale, and passing into the white without any sharp demarcation. On microscopic examination there is often found fatty degeneration of the neuroglia and of the capillary blood-vessels. The. ventricles are sometimes of the normal size, but are often more or less increased in size, and occupied by a correspondingly increased quantity of fluid. The white substance shows on its cut surface no blood-points, or only a few small ones. and is of a milk-white 
colour. The choroid plexuses are strikingly pale, and the brainmass generally deficient in moisture, even the sinuses of the dura mater containing but little blood-generally a pale fibrinous clot.

Partial anæmia of the brain is observed only as the result of tumours and of thrombosis or embolism of the blood-vessels.

Symptoms.-The group of symptoms belonging to cerebral anæmia varies as it occurs in the acute or chronic form, and differs also somewhat according to the age of the patient. In the course of exhausting diseases general anæmia is of frequent occurrence, and under such circumstances the brain of course partakes of the general impoverishment. Children thus affected present a marked paleness, pinched features, wrinkled forehead, with a prominence of the frontal and temporal veins, and the whole skin of the head is loosely voluminous. The anterior fontanelle is either flat or concave, the parietal bone overlapping the frontal, producing a terrace-like projection, perceptible when the hand is passed over it, and the hair of the scalp is scant and dry. There is generally great restlessness of the head, a constant moving it about as if in search of a comfortable resting place for it, accompanied by distortion of the features and frequent rubbing of the head and face with the hands, and plucking at the hair, the eyelashes, ears, and nose. The eyelids are generally only half open, and the pupils are at first contracted, but afterwards dilated. Great restlessness alternates with apathy, sleep is short, and the child wakes up from it with wide staring eyes, and probably soon again relapses into broken slumber. Contractions of the upper, and spasmodic extension and flexion of the lower limbs, together with occasional stiffening of the whole body, form the most important and most frequent brain symptoms. Sudden and frequently repeated vomiting, constipation, and sometimes diarrhœa with watery mucus stools, sometimes streaked with blood, continue up to the time of death. The abdomen is generally distended, doughy, and tender, with the skin wrinkled and in folds.

In some cases the disease runs a rapid though well-marked course in a few days, but in the majority of instances its course is more protracted, and sometimes yields to treatment, though as a rule the result is fatal.

Cerebral anæmia, or hydrocephaloid, appears, as abnve 
described, in infants of one and two years of age, but in more advanced childhood there are essential differences. When it occurs as a sequela of typhoid fever it often induces remarkable mental changes which are generally transient. In such cases children who previous to the attack were of normal mental acuteness, and had good memories become forgetful and feebleminded, the cxpression of the face loses its intelligence and looks imbecile; their replies to questions consist of a meaningless smile, and they often take delight in amusements unusual to their age. In a word, their mental activity is lessened. Cerebral anæmia occurring in rapidly growing children from disproportionate growth, especially between the seventh and tenth years of life, lessens their mental powers, makes their dispositions peevish, produces frequent headache, giddiness, and mental hallucination, unquiet sleep, with grinding of the teeth; but after awhile these symptoms in most cases will entirely disappear.

The symptoms of partial cerebral anæmia are but little known.

Causes.-The causes of cerebral anæmia may be any of the exhaustive diseases of infancy; but it occurs most frequently in profuse diarrhœa in the course of gastro-enteritis (cholera nostras), follicular enteritis, dysentery from unsuitable and insufficient nourishment, more frequently in artificially reared children than in those fed at the breast, from premature weaning, as a result of severe typhoid, from abnormal growth and development, and from loss of blood. Causes of partial anæmia may be suspected in narrowing of the afferent vessels, likewise in any other condition which diminishes the capacity of the skull (tumours, serous effusion, hypertrophy of the retaining membranes, and sclerosis of the brain).

Diagnosis.-This must in great measure depend on a determination of the cause, especially the precedence of such exhausting influences as have been mentioned. Sometimes hyperæmia of the brain may be mistaken for this condition when there is persistent general anæmia, and it may also be confused with meningitis when the characteristic symptoms of that affection are absent. In young infants purulent meningitis may be confounded with cerebral anæmia from similarity of the appearances by which alone we can form an opirion. 
Prognosis. - We can only predict in this disease with an accuracy in proportion with the possibility of the removal of its causes. Generally it is the more grave the younger the child ; but even in the worst cases it is not hopeless, for the infantile system, when judiciously treated, shows a reactional activity sometimes bordering on the marvellous.

Treatment.-The indications for this are twofold ; first, to counteract the tendency towards mental weakness, and, secondly, to treat the fundamental cause of anæmia. Attention should be paid to the ventilation and temperature of the sick chamber, the latter being kept somewhat above the ordinary $\left(73^{\circ}-75^{\circ}\right.$ Fahr.), as the temperature of children exhausted by disease usually stands low. To aid this warm baths, for a few minutes, and warm friction are useful. Internal remedies should consist of stimulants, as wine in doses of fifteen drops to half a teaspoonful frequently repeated, or weak spirits and water, aromatic spirits of ammonia, or the liquor ammoniæ anisata, the ætherial tincture of acetate of iron, and musk. If the child has been recently weaned, then a good nurse is the most efficient remedy; but if the child has been reared by artificial food, or has been weaned for some time, then the diet must be stimulant, a little beef tea being added to the food, wine administered liberally, and a trial made of pounded raw meat, which will often be found of great use in cases where all other food is returned. When the cerebral anæmia results from hæmorrhage, typhoid fever, or disproportionate growth, the preparations of cinchona or quinine, iron, beer, wine, strong and easily digested food, together with residence in fresh air, form the staple remedies. Any condition which tends to weaken or lower the organic activity is to be strenuously avoided.

In chronic diseases which have an exhaustive tendency, the practitioner must employ such means as tend to ward off anæmia, or to limit it if it has already appeared. To what extent transfusion might be useful in severe cases I can form no opinion, having had no experience of it. 


\section{Hyperamia of the Brain and Meninges}

Hyperæmia of the brain and its membranes is of frequent occurrence, and may be of either primary or secondary origin, or acute or chronic in its course. Its frequency is probably due to the unclosed condition of the skull in early life, and the disproportionately rapid growth of the brain.

Anatomy.-Hyperæmia of the brain or of its membranes is almost always general, seldom circumscribed or local. It is indicated externally by a congestion of the skin and superficial coverings, and by an outlining in dark blue colour of the bones of the skull. In the distended sinuses of the dura mater is found thick dark blood, partly coagulated, and the membrane itself is bluish-red, deeply injected, and tightly stretched. The vessels of the pia mater are injected up to their minutest ramifications, and are convoluted or even varicose. The hyperæmia may be due to mechanical causes, such as enlarged glands in the neck, when it may be confined to the half of the brain corresponding to the side on which the enlargement exists. The brain itself is markedly congested, the sulci appear narrower, the cortical substance of a deeper red, and the white substance scattered with numerous blood-points, which are confluent in some places.

If the hyperæmia have been of long continuance there may be odema of the cerebral substance, with effusion of serum into the ventricles, rarely effusion of blood, and the choroid plexuses are found much congested and sometimes occupied by small cysts. In more advanced children in whom the affection has been chronic, there is frequently found a milky obscuration of the arachnoid and increased size of the pacchionian bodies.

Symptoms and course.-It is not possible to arrange the symptoms of cerebral hyperæmia into a definite group, since they vary with the nature of the exciting cause, with the degree of hyperamia, and the age of the child; and since they may indicate irritation of the brain or compression.

Symptoms of cerebral irritation generally precede those of compression, though the contrary may be the case especially when the hyperæmia is passive, the result of obstruction to the venous return. These are shown in such states by more or less reddening of the face, transient flushing of one or both cheeks, 
injected conjunctivæ, epistaxis, contracted pupils, a distended and strongly pulsating fontanelle, increased temperature of the head, especially of the forehead and occiput, very often a whining, peevish, and restless disposition, aversion to light, together with nausea and vomiting. There are generally headache and delirium in more advanced children, together with great irritability when any noise is made, unquiet sleep, with frequent outcry, grinding of the teeth, slight muscular contractions or even general convulsions, a somnolent or comatose condition, disinclination for mental occupation, feelings of oppres. sion and pain in the head, dilated pupils, rapidly progressing paresis, difficult breathing, and small pulse.

Cerebral hyperæmia often appears suddenly and disappears with equal rapidity, or it may be persistent and lead to the results above described. This is frequently seen in hooping-cough, which seems to act frequently as an exciting cause of cerebral hyperæmia, for in its course we often find marked hyperæmia of the head from obstructed venous return.

Recovery is the more common result of cerebral hyperæmia, though in its course the possibility of such complications as inflammation, hæmorrhagic effusion, and damage to the mental powers, must not be forgotten.

Causes.-The disease may arise from relaxation of the walls of the blood-vessels (vaso-motor paralysis), and of the parenchyma of the brain; from alteration of the composition of the blood (toxæmia), from obstruction to the venous return, but rarely from any local affection of the blood-vessels, though fatty degeneration of them is not unfrequently seen in children. As one or other of these known causes is in action we may discriminate the hyperæmia as active (increased flow of blood to the brain), or passive (obstructive) congestion. Active hyperæmia occurs in all feverish diseases as a result of increased temperature, also during dentition, after subjection to high temperature, as insolation or excessive mental effort; finally, in the course of some of the acute exanthemata, as scarlet fever, measles, smallpox, typhoid, and diphtheria. In these diseases the toxæmia may be taken into the reckoning as well as the increased temperature. Mental irregularities, especially during the period of sexual development, may also be traced as the causes of cerebal hyperæmia; and so may in some instances the 
abuse of stimulants and narcotics. Passive hyperæmia occurs in new-born children as a result of severe labour, also from obstruction to the circulation either in the heart or lungs, from laryngeal croup, hooping-cough, croupous or catarrhal pneumonia, severe exudative pleurisy, congenital deficiency in the heart, or acquired valvular disease and rachitic alteration of the thoracic walls. Mechanical hindrances to the return of the blood from the brain, such as tumours in the neck, enlarged lymphatic glands in the neighbourhood of the great vessels, extreme hypertrophy of the tonsils, may likewise induce passive hyperæmia ; and so also, finally, may koprostasis, swelling of the liver, frequent and long-continued clonic and chronic muscular contractions.

Diagnosis.-In addition to the symptoms which have been detailed attention must be paid to the exciting cause, in order that a simple hyperæmia may not be mistaken for a serious cerebral lesion. An accurate estimate of the cause, together with the transient and changing character of the symptoms, the absence of marked and continuing paralysis, especially paralysis limited to certain nerve trunks, is usually sufficient to establish the diagnosis. In addition there is often the simultaneous appearance of indications of some other affection; though it must be remembered that it is no easy matter to explain cerebral symptoms at their onset in any case, still more in children during the first three years of life.

Prognosis.-This will always be favorable in cases where the cause of the hyperæmia has been removed, or is capable of removal, and the balance of the circulation can be restored. It will be bad, on the other hand, when these conditions cannot be secured, or if it has been so long in existence that serious organic alterations of the brain may be suspected.

'Toxæmic hyperæmia, and that resulting from insolation, may involve immediate danger to life, though, as a rule, the prognosis is more favorable here than in passive hyperæmia.

Treatment.-'This may be directed, in cerebral and meningeal hyperæmia, either to the causes or it may be symptomatic, the litter being of course the most important in ajoubtful or urgent cases. All articles of clothing that may be so tight or so arranged as possibly to interfere with the circulation must be removed, and during sleep the head is to be slightly raised 
and kept cool by the application of cold wet cloths or an ice bag. Sometimes benefit is obtained from the method of derivation, by rubbing some part of the trunk or the extremities with some stimulant liquid, as diluted spirit of mustard or infusion of horse-radish.

Evacuation of the bowels by irritant clysters or by the administration of half a grain to a grain of calomel, is often of material assistance. For this purpose the Potio Viennensis may be used.

Bo Fol. Sennæ, క̌ss;

Aq., ว̌iv ;

Sodæ et Pot. Tartratis, そ̧ss ;

Mannæ, jvj.

Doses $\mathfrak{j} \mathbf{j}$ ad $\mathbf{z} j$ ter die.

When convulsions occur oxide of zinc may be prescribed, either alone, in quarter or half-grain doses, or in combination with half-grain or grain doses of calomel. Depletion in children had always better be avoided. When indications of depression occur, as after insolation, in severe paroxysms of hooping-cough, or in the course of pneumonia, stimulants must be administered, as the liquor ammonice anisata, wine, camphor, musk, warm baths, and rubbing with hot vinegar. Treatment directed against the cause of the hyperæmia may consist of the administration of various drugs, or even of operative measures.

\section{Hamorrhages into the cranial cavity-meningeal and cerebral}

Hæmorrhage into the cranial cavity does not often come under our notice, at least if we put out of consideration the occurrence of meningeal apoplexy in new-born children, because the two predisposing conditions of fragility of the vessels and disproportionate force of the arterial blood-current are not so well marked as in later life. Atheromatous degeneration, severe forms of heart disease, cerebral atrophy, and encephalitis are the most frequent causes of apoplexy, and are rare in infancy.

All intra-cranial hæmorrhages may be arranged under two divisions, based on anatomical grounds, and we discriminate intermeningeal and cerebral hæmorrhages according as the ex- 
travasation occurs (1) from the vascular membrane and into the cavity of the arachnoid, or (2) into the cerebral substance. Both forms may, however, occur simultaneously. Cerebral apoplexy may be capillary, or may occur as a large hæmorrhagic centre.

Anatomy.-Intermeningeal hæmorrhage may occur in varying extent from the size of a pea to a florin or larger, and is found most frequently towards the base of the brain, especially towards the posterior lobes (Bednar), affecting the convexity of the brain more rarely. In proportion to the period of its existence the effused blood may be fluid, coagulated, or it may be partly, or for the greater part absorbed, and its remnant be recognised only as a rusty-brown or dirty-looking pigment.

Extravasations are sometimes of great extent, amounting to between one and five ounces, and may extend into the arachnoid cavity of the spine. Occasionally the hæmorrhage is found enclosed in a cyst, and $I$ have seen an instance of this in a case of pachymeningitis.

Capillary apoplexy is seen as small points, varying from the size of a pin's head to that of a millet-seed, easily dispersed, or as thin strata in the spinal marrow or brain substance, and the latter is not unfrequently found in a softened condition.

The clot of an apoplexy arising from rupture of a large vessel may be round in shape or somewhat elongated, and may vary in size from that of a pea to that of a walnut. It may be single or there may be several isolated extravasations, and in position they may be central or peripheral.

Hæmorrhage into the ventricles of the brain occurs exceptionally.

The limitary membranes, cysts, or cicatrices of apoplexies have not hitherto been the objects of anatomical investigation. In many cases, especially in new.born children, apoplexy is only the indication of some alteration in the brain, while in others there occur coincidently embolism, thrombosis, encephalitis, pachymeningitis, hydrocephalus, tumours or atrophy of the brain.

Symptoms and course.-Meningeal extravasations of small extent, as a rule, are of little or no clinical importance; when of large extent, especially when they occur into the sac of the arachnoid, they generally produce symptoms of compression. 
The child lies in a condition of light slumber, does not cry, or emits at most a slight whimper; the face is bluish, and, like the rest of the body, feels cool. The anterior fontanelle is tense, more or less arched, and pulsates at first strongly, but the force of its pulsations gradually diminishes, the pupils are strongly contracted, the cornea somewhat cloudy or even softened, the eyes move tremulously, the extremities are either perfectly paralysed or are found in a state of slight contraction to which convulsive movements are occasionally added. The pulse is small and slow, the respirations are carried on slowly, and are superficial in character, with occasional deep efforts. The alvine evacuations are either normal, or there may be diarrhœa, accompanied by vomiting. The child does not readily take the breast, and soon lets loose its hold, or it may be unable to seize the nipple from the presence of trismus. The duration of these cases is from four to eighteen days, though it may be influenced by the coincidence of other diseases.

The occurrence of an apoplexy of large size is not so often seen in new-born and suckling children as in the later years of infancy, is generally sudden, and may take place in a child previously enjoying perfect health, or after only slight premonition, such as headache, great irritability, or unquiet sleep. As a rule, it does not uccur with the same severity and with the intensive lesions met with in adult and senile life. Transient unconsciousness, diminished or entirely arrested perception, paralysis of isolated muscle-groups, especially unilateral, slight or more severe convulsive attacks, loss of speech, strained movements, contractions, retarded action of the heart, irregular noisy breathing, form, in variously modified ways, the symptom-complex of cerebral hæmorrhage. Since idiopathic apoplexy is rare in infancy, the range of symptoms will be multiplied and altered by other cerebral complications. Capillary apoplexy, if small in extent, may escape observation from want of any pronounced symptoms; when more serious, I have often seen it ushered in by convulsions and great restlessness, followed subsequently by stupor lasting up to the time of death. In some cases the first indication is found in paralysis being noticed by the parents. The subsequent results of cerebral hæmorrhage are encephalitis and softening of the substance surrounding the clot, and a more or less obstinate paralysis. 
Causes.-We find cerebral hæmorrhage occurring most frequently in the new-born and during the first four weeks of life; between that time and puberty it occurs only in exceptional cases. It is also met with somewhat more frequently in male than in female children. Laceration of minute vessels, producing hæmorrhage, is due sometimes to local, sometimes to remote causes; of the former, fatty degeneration of the coats being an uncommon one, while in some cases of apparently healthy children the hæmorrhage seems to be due to extremely slight causes, or even to be causeless. Local causes are more commonly instanced in hyperæmia of the brain and its membranes, thrombosis of the sinuses and meningeal veins, embo. lism by the impaction of dislodged vegetations or clots in heart disease or of pigment (Pigmentauschwemmung), tumours of the brain, encephalitis, pachymeningitis, hydrocephalus, injuries of the bones during birth; whilst asphyxia, congenital abnormalities of the heart and great vessels, acquired heart disease, hypertrophy of the thyroid and thymus glands, enlarged and - tubercular bronchial glands, especially in the neighbourhood of the heart, hooping-cough (Löschner), trismus and tetanus neonatorum, umbilical gangrene with subsequent peritonitis, nephritis albuminosa, and such hæmic alterations as pyæmia, scarlatina, measles, smallpox, typhoid fever, and purpura, may be mentioned as remote causes to which cerebral hæmorrhage in childhood may be due. The influence of the acute zymotic diseases in producing cerebral hæmorrhage may probably be referred to the accompanying hyperæmia of the brain; also to the disturbance to the nutrition of the vessels-at least some of my own cases would point to that conclusion; and acute fatty degeneration is found in other organs, as the heart and liver, during the course of these zymotic affections.

Prognosis.-If the hæmorrhage can be diagnosed with certainty, or at least with probability-neither of which conditions are easy, however-and the circumstances of the constitution of the affected child and severity of the symptoms be favorable, an opinion may be given that, in the case of extravasation of small extent, recovery may take place without permanent detriment. Extensive hæmorrhages, on the other hand, are almost invariably fatal, especially in the intermeningeal apoplexies of newly born children. Paralysis resulting from apoplectic clots, I have 
known, in several cases to disappear only after an interval of two or three years; while in other cases all treatment has been futile, and atrophy of the paralysed limbs has resulted.

Treatment.-A knowledge of the causes alone may convince us that no treatment will have any very brilliant result, and since the influence we can bring to bear on the exciting causes is usually limited, we have almost to confine our efforts to symptomatic treatment, consisting of such remedies as are used in hyperæmia of the brain and its membranes. Care should be taken to ward off all influences likely to induce hæmorrhage. The treatment of the resulting paralysis will be found in a special chapter.

\section{Thronbosis of the Cerebral Sinuses}

Coagula form in the sinuses of the dura mater under many various conditions, either as primary or secondary lesions, and sometimes may be recognised clinically as independent affec-. tions, sometimes as concomitant with or subsequent to other cerebral diseases, with the symptoms of which they are more or less blended.

Anatomy.-The coagula which occupy and distend the affected blood-channels are generally found to be firm or even hardish, of a brownish-red or yellow colour, their surface sometimes smooth, sometimes uneven or slightly tuberculated, and with their free ends conical or rounded. They may be attached throughout their whole length, or only at one point, to the internal wall of the vessel, or they may lie quite free in the cavity. Decoloration, softening, or ichorisation of the clot, and even obliteration of the vein, are not unfrequently found as after results of the coagulation. The most frequent seats of the coagula are the transverse and straight sinuses, less frequently the longitudinal, and most rarely the cavernous, petrous, and circular sinus (Gerhardt). In fourteen cases I found the thrombosis seven times in the longitudinal sinus, twice in the longitudinal and transverse sinuses, once in the longitudinal, transverse, and sigmoid sinuses, thrice in the transverse sinus, and twice in the sigmoid sinus. In five of these cases the meningeal veins were more or less occupied by clot, in three there was chronic hydrocephalus, in three pachymeningitis, in 
two there was internal hæmatoma, in another two intermeningeal apoplexy, in one thrombosis of the jugular vein, and in the remaining instance there was metastatic embolism in the lungs and heart.

In these children other diseases were present, as acute and chronic gastro-intestinal catarrh, general tuberculosis, caries of the petrous bone, pyo-pneumothorax and gangrene of the lungs, rickets, pneumonia, and repaired fracture of the skull.

Symptoms.-In cases of uncomplicated cerebral thrombosis, that is, in cases when no other cerebral lesion is present, symptoms of obstruction to the cerebral circulation may be observed occasionally by which a diagnosis can be made; but, on the other hand, when other lesions are present, it may be difficult or even impossible to determine its presence. The best instances of uncomplicated cerebral thrombosis are met with in cases of exhausting diarrhœa, in cholera infantum following follicular enteritis. In such cases the symptoms consist of, besides general wasting, intercalation of the bones of the skull, sinking in of the anterior fontanelle, clouded consciousness, lethargy, deep stupor and signs of oppression, paralysis in the region of the facial and oculomotor nerves, stiffening of the muscles of the neck and back, \&c. ; and indications of coagulation may be found in other regions, which may lead us to suspect the possibility of its occurrence in the cerebral sinuses.

The following results have been frequently confirmed by experience :

I. If the canal of the transverse sinus, or perhaps with the inferior petrosal sinus, or the point of origin of the internal jugular vein, be occluded, and the veins of the neck be found generally distended, the external jugular of the affected side will be found emptier than that of the sound one (Gerhard and Huguenin).

II. If the coagulation passes through the aperture of the papillary appendage out of the transverse sinus to the posterior auricular veins, there is formed behind the ear a circumscribed, hard, œdematous swelling (Griesinger and Mohs).

III. The plugging of the cavernous sinus affects first of all the ophthalmic vein, which empties most of its blood into that sinus, and there results hyperæmia of the veins of the orbit, as shown on ophthalmoscopic examination, and subsequently 
slight exophthalmos (Huguenin), œdema of the conjunctiva," or of the eyelids and whole side of the face (Genouille).

IV. Coagulation in the cavernous sinus may produce symptoms of irritation or paralysis from direct pressure, of the first division of the fifth, or of the nerves supplying the orbital muscles (Heubney).

V. Thrombosis in the superior longitudinal sinus produces cyanosis of the face, enlargement of the venous radicles or reticulæ which extend from the great fontanelle to the temporal regions, circumscribed perspiration of the forehead and nose, and epistaxis, as has been noticed frequently by Dusch and myself.

The case may run its course in a time between a few days and three weeks, though it is not always easy, especially in chronic exhausting diseases, to recognise the beginning of thrombosis. The other forms of the disease met with resemble either fever of pyæmic character, the cerebral anæmia (hydrocephaloid) already spoken of, or encephalitis interstitialis. The result is almost always fatal, though Griesinger and other observers have recorded cases of recovery, but from my own experience this can have happened only when the coagulation has been very limited.

Causes.-Age seems to be a predisposing cause, for the large majority of cases occur under two years of age, and it has been met with in the new-born. Of fourteen cases observed by myself five children were in the first year of life, four in the second, two in the third, one in the fourth, one in the fifth, and one in the ninth.

The immediate cause of cerebral thrombosis is generally some form of retardation of the blood-current, affecting either the circulation in general or having only a local influence by pressure on the cerebral sinuses; or from inspissation of the blood, as in cholera infantum; or from inflammation of the dura mater, wounds of the skull, or caries of the bones, especially of the petrosal bones. It may be divided into exhaustive and inflammatory, the first occurring in acute and chronic gastro-intestinal catarrh, in tuberculosis, scrofula, rickets, pyopneumothorax, pyæmia, and probably in all exhausting diseases; 
whilst the second form occurs after wounds, in pachymeningitis, and with caries of the petrous bones.

Treatment.-Marantic thrombus, or that threatening from exhaustion, may be obviated by well-timed prophylactic treatment, when possible, by combating the disease which is present before the coagulation has taken place. To prevent the formation of thrombi stimulants and strong food are useful, as wine, strong soup, raw meat, meat juice, a wet-nurse for children who have been weaned, together with such drugs as the etherial tincture of the acetate of iron, musk, \&c. In the inflammatory form an antiphlogistic form of treatment is to be recommended. With all this recoveries seem so rare that their occurrence may almost be doubted.

\section{Pachymeningitis-Inflammation and Hamatoma of the Dura Mater}

Inflammation of the dura mater is seldom a primary lesion, being usually the result of some other process, and it may be external or, more frequently, internal.

Anatomy.-In pachymeningitis externa we find on the outer surface of the dura mater a more or less marked network of vessels, simple punctation, and larger spots of ecchymosis; now and then, also, collections of matter of various sizes. Occasionally, especially in lesions of the bones of the skull, there is developed a vascular netlike osteophyte, which remains adherent firmly to the dura mater, when the latter is stripped off from the bones, or it may be ossified.

Pachymeningitis interna is characterised chiefly by pseudomembranosis, delicate vascular effusions, amongst which hæmorrhages of various extent are found. Some of the branches of the meningeal veins and the sinuses contain thrombi, partly dark red or rusty brown, the latter being the prevalent colour if the inflammatory action be due to caries of the petrous bone. Pachymeningitis may also excite sympathetic inflammation of the pia mater.

Large hæmorrhages between the dura and pia mater produce hæmatomata, which, in the majority of cases, are found in the convexity of the brain, seldomer at the base. "In one case, a child of six years old, I found a roundish-flat cyst, three inches 
broad, with tolerably thick walls and sero-sanguinolent contents, situated in the anterior fossa of the skull, and which had exerted considerable pressure, with resulting atrophy of the cerebral substance.

Symptoms and course.-Pachymeningitis has no certain set of symptoms by which its presence can be recognised with safety. Just as the inflammation is acute or chronic so are the accompanying hæmorrhages sparse or abundant, and the course of the disease varies. At first the range of symptoms resembles that of cerebral hyperæmia, but it may assume the form of meningitis, or even hydrocephalus during the further course or shortly before death. Children of more advanced age complain of pain in the head, affecting, in cases of wounds, the part of the head in the neighbourhood of the wound, and in internal otitis confined to the affected side of the head. Children between one and three years of age betray their sufferings by great restlessness, frequent clutching at the head, crying, and whimpering; nausea, vomiting, slight convulsions, apathy, somnolence, and, at last, complete coma, are met with in the further course of the disease. Sudden hæmorrhages may produce immediate death; and sometimes, even when death is not sudden, no other symptom than somnolence may be observed. The duration of the disease is sometimes short-from one to two weeks-though it may go on for three or four months, or even longer.

Hæmatoma of the dura mater displays itself generally by symptoms of a localised affection of the brain and produces periodically occurring epileptiform convulsions. These convulsions occurred in a child six months old, under my care, at first every two or three weeks, afterwards every week, and finally almost daily, without any sign of paralysis. A case of hæmatoma at the base presented during the last fortnight of life symptoms of tubercular meningitis.

Causes.-Amongst the causes of pachymeningitis and hæmatoma of the dura mater traumatic injury takes the first rank, and after it comes caries of the bones, especially of the petrous bone, inflammation and suppuration of the vessels in erysipelas, pyæmic affections, and general hypertrophy and sclerosis of the brain. It may originate spontaneously in wasted children affected by pulmonary or intestinal diseases or by rickets, being probably excited by marantic thrombi; also in the course of 
acute zymotic diseases. Children between the first and fourth years of life are most frequently attacked, and sex does not seem to exert much influence.

Treatment.-The uncertainty of the diagnosis and the comparative certainty of a fatal result confine all treatment to the indications afforded by symptoms and to the use of such remedies as are employed in cases of meningitis and hydrocephalus. Cold wet applications to the head, derivation from the skin and intestinal canal by counter-irritants and purgatives, and the administration of oxide of zinc or opiates when convulsions occur, form the usual though generally useless treatment. In weak and wasted children, in whom the affection is chronic, good nourishment, with such tonics as quinine and iron, are of course to be tried.

\section{Inflammation of the Pia Mater-Meningitis simplex; Lepto-meningitis}

Meningitis simplex is not a common affection, for out of a thousand cases of brain disease it has occurred only eight or ten times, and is very rarely idiopathic, being generally secondary to some other lesion.

Anatomy.-It - is developed by preference and most frequently on the convex surface of the brain, though it may also be found at the base. The vessels of the pia mater are found strongly injected, with small ecchymoses scattered about, and accompanying the larger veins in their course between the convolutions is sometimes found a greenish-yellow exudation, more or less firm and often purulent. In the rarer cases where it has run to an extreme extent the basal surface of the brain is found bathed in a layer of pus about a line in thickness, and there is often serous, very rarely purulent, effusion into the lateral rentricles. The effusion generally extends from the base down the spinal meninges, and on the convex surface of the brain the inflammation not unfrequently extends into the cortical substance which becomes strongly injected or softened. When the course of the disease becomes chronic the effusion udergoes alteration into a firm milky mass, sometimes cheesy. The sinuses are occasionally occupied by thrombi, grenerally with more or less coagulated blood. 
Symptoms. - The train of symptoms in simple meningitis is usually developed rapidly, and advances with fatal impetuosity. The child, which may have been in a good state of health previously or may have been reduced in health by some exhausting disease, may become suddenly unconscious or be attacked by more or less severe convulsions, following each other rapidly up to the time of death, which may be from thirty-six to forty-eight hours from the beginning of the attack. In sucklings coma usually takes the place of convulsions. Subsequent to these primary indications of the disease there occur symptoms of fever with increased temperature of the head, frequent alternations of colour from pallor to redness, grinding of the teeth, great restlessness, and very often vomiting. Should the convulsions disappear the child falls into a condition of great exhaustion, apathy, and stupor, from which it will be roused by the recurrence of the convuisions. In more advanced children the disease is often ushered in by a well-marked rigor, followed by severe pain in the head, romiting, giddiness, photophobia, with contracted pupils at first and dilatation of them in a later stage. Sucklings and young children frequently carry the hands to the head and give vent to painful cries or to a low whimpering. In the early stages of the disease there are intermittent pauses during which consciousness is present, but these quickly disappear and consciousness is lost completely and for ever. With the convulsions partial or very extensive paralysis is often associated.

If the fontanelles have not closed previous to the attack they will be found more or less distended and pulsating, with an alternating depression and elevation.

The pulse is at first accelerated, but is retarded as exhaus. tion occurs, and so also are the respirations, though not in the same degree nor so constantly as in tubercular meningitis. The abdomen is generally unaltered in form, though it may be distended, tympanitic, and tender, or somewhat retracted. The bowels are usually costive, though they may be normal or even relaxed.

In very young children meningitis simplex may run its course without the occurrence of any of these severe symptoms, its only indication being great depression, taking even the form of coma, the child at first evincing only an apathetic condition, which 
passes into coma, and is interrupted by a single convulsion shortly before death, or no convulsion may occur. The bulky exudation found in such children explains the severity of the symptoms of depression. In purulent, especially in traumatic meningitis, there frequently occurs more or less abundant purulent discharge from one or both ears, with subsequent indications of severe irritation.

The duration of meningitis simplex varies from two to fourteen days, and recoveries are rarely met with. Interference with the mental activity, deafness, blindness, muscular contractions, or paralysis, form, in the rare recoveries, frequently melancholy and life-long sequelæ of the disease.

Causes.-Meningitis simplex occurs in children at all periods of life up to the time of puberty, and may also occur during intra-uterine life, sex seeming to exercise no influence on its frequency. The primary form is met with in well-nourished children as well as in the badly nourished, as a result of insolation, cold, injury, excessive mental effort, though very frequently no cause can be assigned for the attack. Secondary meningitis occurs in erysipelas of the face and scalp, in the acute exanthemata, typhoid pneumonia. occasionally in Bright's disease, in pyæmic affections, especially in new-born children and sucklings, as a consequence of pachymeningitis, internal otitis, ozæna, periorbitis, coxitis, chiefly of scrofulous origin.

As causes of intra-uterine meningitis, I have found such diseases of the mother, during pregnancy, as variola and puerperal fever.

Diagnosis.-An accurate estimate of the previous history and ætiology in connection with the rapid onset and course of the disease are the leading features to be considered in the diagnosis. Indications of scrofula or tubercular disease in children in whom the symptoms are developed more slowly ought to give rise to suspicions of tubercular meningitis. A doubt between typhoid fever and meningitis during the first few days of the attack is not unfrequent, but the differential diagnosis can be made subsequently by the appearance of the characteristic roseoloid rash, the enlargement of the spleen, and the occurrence of bronchitis and diarrhœa; also by the exacerbation of the fever at evening, with a remission towards morning. It is not impossible to mistake meningitis for the so-called cerebral pneumonia 
at its commencement, but this will easily be obviated by a physical examination of the chest. Cerebral hyperæmia does not excite such intense symptoms as meningitis, and is generally evanescent. In brain affections depending on uræmia, which sometimes in infancy closely resemble meningitis, the characteristic suppression of the urine and the occurrence of dropsy, are sufficient to prevent mistake. It is not always possible to differentiate the comatose form of simple meningitis from cerebral anæmia, though a careful investigation of the exciting cause and the general habit of the affected child, together with the fact that in meningitis the symptoms of fever are more marked than in cerebral anæmia, may be sufficient to clear up the difficulty.

Prognosis.-Recovèry is an exceptional result in this disease, death being the rule; the prognosis is, therefore, almost always unfavorable, though the possibility of recovery must be conceded. Meningitis occurring in the course of a pyæmic affection may be looked upon as certainly fatal. If recovery from simple meningitis should occur it is unlikely to be complete, for there will probably remain behind some lesion of the motor, or mental functions.

Treatment.-At the commencement of the disease, antiphlogistic remedies are to be employed. Abstraction of blood, whether local or general, will not produce the beneficial results claimed for it by certain authors, and is strongly contra-indicated in weakly, reduced, and anæmic children. Local applications in the form of wet packing or ice-caps act more in a calmative than in a curative manner. The application of mercurial, iodine, or irritant ointments to the shaved head seems to have little influence over the course of the disease, and they are always very problematical remedial attempts. Just as little is to be hoped from the internal administration of iodide of potassium. Constipation is to be relieved by such laxatives as the Vienna mixture, calomel, or by clysters. In delirium or frequently recurrent convulsions, swathing the whole body in cold wet packing is frequently useful, and the administration of opium or morphia, either by the mouth or by subcutancous injection, may be tried.

When symptoms of severe depression appear, stimulant remedies, as camphor and musk, are to be given, though from them 
there is little hope of any good result. In the pyæmic form the custom of giving quinine in doses of half a grain to a grain is in fashion.

Absolute rest, with a perfect absence of all disturbing influences, and free ventilation of the sick room are matters of course. Should the disease tend to take a chronic form, with remission of the graver symptoms, then stimulating diet and tonic remedies are to be adopted. For the sequent lesions of the mind or nervous system therapeutics can do little or nothing.

\section{7.-Meningitis Tuberculosa, Basal Meningitis ; Tubercular Inflammation of the Membranes of the Brain}

The essentials of this disease consist of tubercular granulations with simultaneous exudation into the pia mater, especially at the base of the brain, together with acute hydrocephalus. The inflammatory condition stands to the granulations in the relation of cause, and it may exist for some time before the meningitis and hydrocephalus occur. In cases where the latter is not produced the lesion is found simply as a miliary tuberculosis. Tubercular meningitis I have found 224 times out of 4292 cases of brain diseases.

Anatomy.-The characteristic changes are met with at the base of the brain in the form of masses of exudation on the meninges. The most frequent seat is the chiasma of the optic nerve, extending backwards to the medulla and forwards on the convolutions, diminishing as it recedes from the above-mentioned spot. These granulations are about the size of a pin's head, and are derived, according to Lebert, by cell-growth from the lymph-sacs of the arterioles, being found chiefly in the course of the vessels accompanying them, especially in the Sylvian fissure, sometimes to such an extent as completely to bridge them over. These tubercles are rarely found on the meninges of the cerebellum or upon the convexity of the cerebrum. The ventricles are usually increased from three to six times in size, and are occupied by an increased quantity of serum, either clear or somewhat cloudy or flocculent, the commissures are much softened and easily torn, and the ependyma generally loosened, softened, and easily stripped off. On this membrane 
the tubercular granulations are sometimes found, or even there may be little hæmorrhagic clots. The choroid plexuses are generally of a pinkish colour, and scattered over with granulations. The substance of the brain is white and deficient in blood, that of the cerebellum betng more anæmic than that of the cerebrum. In the sinuses of the dura mater are found partly free clots, partly thick blood; the dura mater is tightly stretched, the outer surface of the brain much levelled, the sulci being closed up, and the fornix arched forwards, and large inflammatory centres, greenish-yellow in colour, may be met with in the cortical substance or in the central ganglia. Tubercular granulations have been noticed by Cohnheim on the vascular tunic of the retina, and miliary tubercles, as also tubercular masses of considerable size, have been found coincidently in other organs, as the glands, the lungs, the liver, spleen, \&c. In some rare cases there is no other trace of tubercle found in the body than in the brain, the tubercular meningitis being the first and only sign of tuberculosis. Great dryness of the dark red substance of muscle and of the subcutaneous cellular tissue, also softening of the stomach, are frequent, though not constant, post-mortem appearances.

Symptoms and course.-Neither the symptoms nor the anatomical facts allow of the division of the course of the disease into stages, for it is developed insidiously, and is often ushered in by symptoms apparently of little importance, but which, to the experienced eye, have grave indications.

Among the prodromata are found a general wasting of the body, withering of the skin, and a striking alteration in the demeanour of the child. It becomes peevish, morose, and capricious in disposition, inclined to solitude, and evinces a distaste for its accustomed amusements, a dislike for loud noises, a timid and spiritless humour, and weariness is easily induced on the slightest bodily exertion. There are also indications of absence of mind and loss of memory, together with restlessness and unquiet sleep broken by startings and cries. The child also gives indications, of pain in the head, pain and stiffness of the neck, unsteady and tottering gait, decrease of appetiteall these forming with manifold variations the initial symptoms of the disease.

The course of the disease extends from two to four weeks, or 
even longer, and when the symptonis steadily increase in severity it can scarcely be mistaken, though repeated vomiting is often the first indication which gives rise to alarm, occurring without nausea or over-eating, and the vomiting does not usually occur in the later progress of the disease. From this period the child is generally confined to bed, or may leave it only for a short time; the appetite disappears, and thirst is much increased. The pain in the head is usually severe, evinced by cries, and there is usually a sluggish action of the bowels or constipation, save in those cases where diarrhœa exists, due to. tubercular disease of the intestines.

The tongue is dry, with a yellowish or dirty white fur; the urine is scanty, and contains an excess of phosphates. The skin is often markedly hyperæsthetic, the slightest touch being painful.

An increase of temperature is always present, though some: times it affects the head exclusively, while the temperature of the body may be normal, and that of the lower extremities even diminished. Variations of the temperature, especially of the head are frequent, a high temperature and clammy perspiration occurring usually just before death. At the onset of the disease the pulse beats are from 120 to 140 in the minute, but afterwards they fall to between 60 and 80 , or even lower, rising again towards the close of the case sometimes to as many as 160 beats in the minute. A similar variation is ob: served in the respirations, these being sometimes accelerated and sometimes retarded, sometimes superficial and sometimes deep, accompanied by a sighing noise. The child is generally apathetic, inclined to sleep, though towards night it becomes restless and emits peculiar sharp cries, and clutches at its head frequently with its hands, turning the head hither and thither and digging it into the pillow. There. is often grinding of the teeth and retching.

$\Lambda$ t a later stage the child lies with half-opened eyes, with a more or less marked squint, and there appear also various lesions of motor power, as partial or general clonic contractions, tetanic convulsions, bending of the head backwards, and trismus.

Paralysis occurring in this disease is the result, probably, of pressure on the brain, and occurs in various forms, being fre- 
quently first indicated by photophobia and contracted pupils, and subsequently by dilatation of the pupils and an absence of reaction on the stimulus of light, together with an increased flow of tears with strong injection of the conjunctiva, and sometimes keratomalacia in one or both eyes.

The presence of tubercle at the fundus of the eye (Gräfe, Bouchut) is a frequently, though not constantly, observed condition, the ophthalmoscopic examination of children being not always an easy matter. Hearing becomes also affected later on in the course of the disease.

When tubercular meningitis occurs in a child in whom the fontanelles are still unclosed, these are seen to be distended throughout the course of the disease. The navicular retraction of the abdominal wall obscrved in all cases with few exceptions, is neither to be regarded as contraction nor paralysis of the abdominal muscles, but has not yet had a satisfactory explanation. Traube has suggested that it may be due to an increase of the tone of the circular fibres of the intestine, by which flatulence is expelled or prevented, and retraction of the abdominal walls results. There is a nerve centre in the neighbourhood of the medulla oblongata which appears to control the whole. of the circular fibres of the intestine.

The so-called meningitic spot (tache cérébrale), which is seen as large evanescent wheals following scraping with the nail on the skin, is often met with. Frequent seizing and pulling at the genitals is sometimes observed, due to the irritation of the pudendal plexuses.

The duration of tubercular meningitis may be, in the more rapid cases, ten to fourteen days; when more prolonged, from four to six weeks, though a fortnight or three weeks may be taken as the average. The actual duration can seldom be accurately determined on account of the insidious appearance of the initial symptoms.

Causes.-The disease occurs most frequently between the first and seventh years of life, seldom in the first year. Boys are more frequently attacked than girls. In relation to the season of the year, cases occur most frequently in April and May, somewhat seldomer in January, February, and March, and with an average frequency during the other months of the year. 
Tuberculosis and scrofula form the most frequent and important causes. Poverty and residence in populous towns favour, without doubt, the outbreak of the disease.

The opinion that meningitis is always a resorptive process, and arises from cheesy, tubercular, or purulent centres (Waldenburg and others) has not met with general acceptance, and is rendered improbable by the occurrence of occasional cases where the meningitis is met with alone.

As occasional causes of the outbreak of the inflammation may be mentioned measles, hooping-cough, typhoid fever, and internal otitis. An erroneous opinion is very popular that the disease often occurs from the too rapid healing of chronic, eczematous, and other eruptions of the scalp. If such should occur the explanation is to be sought in the absorption of purulent detritus.

Diagnosis. - When the disease is fairly established there is little difficulty in the diagnosis, though at its commencement its discrimination is by no means easy. From simple meningitis it is to be distinguished by its prolonged course, the prodromata, the ratio of the pulse, the condition of the abdomen, the previous or simultaneous presence of indications of scrofula and tuberculosis in the patient, or hereditarily in the family. The presence of granulations at the fundus of the eye is specially important and decisive, so much the more if there existed symptoms of general tuberculosis before the onset of the cerebral symptoms (Fränkel).

The possibility of mistaking tubercular meningitis for typhoid fever, and vice versấ is great; but it may be avoided by looking for some of the peculiarities of the latter disease. It sometimes may require the greatest care and skill to determine between a case of tubercular meningitis and one of chronic hydrocephalus complicated with rickets in a child between two and four years of age. From cerebral pneumonia the disease may be distinguished by the high temperature $\left(40^{\circ}\right.$ or even more), by its remission at the critical periods, and by the evidence of consolidation in one or both lungs. By the absence of serious brain symptoms it is easy to mark the distinction between it and an acute intestinal catarrh. Helminthiasis seldom imitates tubercular meningitis closely, though the diagnosis may be 
very difficult when it supervenes in children already suffering from the presence of worms.

Prognosis.-This is always unfavorable, for this disease nearly always ends fatally. Isolated instances of recovery may be quoted, but they are not fully established.

Treatment.-Though the treatment can afford no gratifying result, we are bound to do what is possible; and though we can do little to cure, yet we may do much to mitigate the pain of the disease and the distress of the friends. The chief remedies are cold applications and the administration of opiates, which may be employed in much the same ways as were recommended for simple meningitis. The use of quinine, the iodide of potassium, and iron, \&c., has not yet proved of much service. In advancing collapse the use of the routine remedics, such as musk, ether, camphor, \&c., finds its apology only in the helplessness of the practitioner and the distress of the parents. Though so little can be done when the disease has become thoroughly pronounced, yet a well-timed and efficient prophylaxis may be of great service. All remedies which are calculated to do battle with the scrofulous and tubercular conditions are indicated as those likely to be useful in warding off tubercular meningitis, and of those the best are cod-liver oil, iodine, iodide of iron, and a rational, physical, and mental education. All procatarctic causes, as insolation, hooping-cough, measles, in tubercular children, are to be carefully watched and treated.

\section{Encephalitis; Inflammation of the Brain}

Inflammation of the cerebral substance, either simple or purulent, in infancy does not come often under our observation; though, if we take into consideration the affection recently made known by Virchow under the term of Encephalitis interstitialis, the frequency of the disease is considerably increased.

Anatomy.-Simple encephalitis occurs in the cortical substance as well as in the mass of cerebrum or cerebellum, and is met with sometimes in scattered or isolated spots, sometimes diffusely spread, this being especially the case when it attacks the cortical substance. During the progress of the disease the inflamed brain substance may become either softened, which is 
the more frequent process, going on to absorbtion and atrophy, or sclerosis of the tissues may occur, Remains of intra-uterine encephalitis are sometimes found in the form of isolated or closely grouped nodules in the substance of the great hemispheres, varying in size from a millet seed to a grain of corn, with cavities in their centres. I have also found such associated with recent encephalitis and meningitis. Inflammation resulting in abscess is found in all parts of the brain, though it is most common in the peripheral parts of the cerebral hemispheres and in the cerebellum, the latter especially as a result of internal otitis. Ecchymosis of the affected centres, inflammation of the surface of the brain corresponding to the position of the purulent deposit, and thrombosis of the sinuses, are often found in conjunction with abscess.

The interstitial encephalitis of Virchow is met with as a parenchymatous inflammation which subsequently passes on to fatty degeneration of the neuroglia, and it has as its chief seats the cerebral hemispheres and spinal cord. Under the microscope the neuroglia-cells of the affected spots appear as darkish points, and these centres may increase to the size of small grains of corn. If these points of fatty degeneration accumulate in number, there appears a white, opaque, or yellowish-white, faintly marked spot, perceptible to the naked eye, which may be of as large an extent as half an inch in measurement. I have seen coincidently with this interstitial encephalitis, softening of the stomach in a child six weeks old.

Symptoms and course.-Inflammation of the brain substance may remain latent a long time, though, as a rule, indications soon present themselves of serious brain lesion. The train of symptoms chiefly resembles that of ordinary purulent meningitis, with this difference, that the course of the encephalitis is more prolonged, especially in its earlier stages, and is often marked by longer or shorter remissions. The mental powers are often remarkably little interfered with until close to the end of the case. This I have noticed especially in abscess of the cerebellum, in which the children complained of extreme pain in the occipital region, rising occasionally to extreme agony, the paroxysms being now and then followed by convulsions. If the pus can make its way to and have an exit at the surface, as may happen after injuries, the cerebral symıtoms may be a long time 
in abeyance, even when there has heen a large destruction of brain substance.

A remarkable case came under my notice, in a child five years old, with a gaping fracture of the skull, in whom the greater part of the left cerebral hemisphere, partly by suppuration; partly by necrosis, was destroyed, the brain substance protruding through the gap and sloughing off, yet for three weeks not a single brain symptom appeared. The boy when awake sat up in bed and ate his meals with good appetite, and could sleep well throughout the whole illness. Only at the end of the third week came a suppurative meningitis and carried him off in two days.

Interstitial encephalitis, an affection only recently recognised, is accompanied, so far as my own experience goes, by very much the same train of symptoms as is met with in anæmia and atrophy of the brain (bydrocephaloid). This form of brain inflammation is an intra-uterine one, and is met with in deadborn children or in those who die during the first week of life, though it may also be developed in later life. I' have found the following among the symptoms:-Deficient development or growth, dry skin, its temperature being low especially in the extremities; the pulse small, 120-140, and in the later stages no longer to be felt; the frontal and occipital bones wedged in between the parietal, the fontanelles sunk in, the cornea on both sides is at first somewhat dimmed, faintly glittering, and afterwards becomes softened. The eyelids remain half opened, the breathing is superficial, there is frequent vomiting, together with refusal of the breast and other nourishment, convulsive drawing up of the extremities, especially of the lower on to the abdomen. Great disquiet, with outcrying, succeeded by painful whimpering, apathy and, finally, complete coma, characterise the last stage of the disease.

Causes.-Inflammation of the brain occurs frequently as an intra-uterine affection, and this refers especially to the interstitial encephalitis. Other causes, especially in older children, are furnished by traumatic lesions, internal otitis with caries of the petrous bone, suppurative meningitis, tumours of the brain, hæmorrhage into the cranial cavity, pyæmia, scrofula, and syphilis. As causes of the acquired interstitial encephalitis are mentioned by Virchow, the acute exanthemata, especially smallpox and syphilis, and I have conjectured that we may add to these 
intestinal catarrh, especially of a very acute form; and further examination will, no doubt, reveal other ætiological factors.

Prognosis.-This is generally unfavorable, for even if recovery takes place it is usually marked by some loss of mental or motor power.

Treatment.-In traumatic encephalitis an antiphlogistic treatment is to be employed chiefly, as cold applications to the head, deriratives from the skin and the intestinal canal, together with absolute mental and bodily rest. To mitigate the severe pain and nocturnal restlessness opiates by internal or by subcutaneous administration may be used. In frequently occurring convulsions oxide of zinc, bromide of potash, and cold packing are customary. The interstitial encephalitis requires, if it is recognised, a tonic treatment, though it is almost always futile.

\section{Hypertrophy and Sclerosis of the Brain}

True cerebral hypertrophy, which does not consist of an increase of the cerebral substance, but of a hyperplasia of the neuroglia, does not often occur in infancy, and the method of its production is still obscure. It is often associated with sclerosis, and clinically they cannot be separated, and we may therefore consider them together, though, as I have already observed, partial sclerosis occurs with atrophy of the brain.

Anatomy. - The skull is generally enlarged, as is found in hydrocephalus. I have found its circumference as much as twenty-nine inches in a child ten months old. The dura mater is tightly stretched and arched outwards at its apertures; the membranes are dry and closely adherent to the brain, and the cerebral hemispheres have a disproportionate relation to the volume of the cerebellum. The sulci are filled up and the convolutions flattened, the cortical substance of a pale red colour, the white substance dull white and scattered with sparse blood-points, the brain substance also unusually dense, especially in the semi-oval centre of Vieussens, firm, and sometimes of an almost cartilaginous hardness from the sclerosis, and glistening like tendon. The ventricular walls are in close apposition. In one case I found pachymeningitis associated with marked hypertrophy and sclerosis of the brain, and, in 
some rare cases, sclerosis of the cerebellum, the pons and cord have also been found.

Partial sclerosis may occur in one or more greater or less centres, and at these spots the brain is found to have a perceptible increase in thickness, or to have a cartilaginous hardness, and the convolutions are often reduced to a half, or even less, of their normal thickness.

Symptoms and course.-Hypertrophy and sclerosis of the brain have no symptom-complex belonging exclusively to them, for the indications of the cerebral disorder vary in proportion with the pressure to which the brain is subjected. These symptoms gain the ascendancy towards the close of the case; and before they appear the child is generally much reduced in health. When the skull is still unclosed, on the access of the disease the diameters are much increased, and the head assumes the form seen in hydrocephalus. Occasionally the mental activity is not at all affected, but often there is complete imbecility, somnolence, or great restlessness, with frequent crying out during the night, pain in the head, hallucinations, blindness, widely dilated and sluggish pupils, nystagmus, strabismus, dulness or total absence of consciousness, vomiting, irregular respiration, retardation of the pulse, trembling of the extremities, especially when they are touched, convulsions, trismus, tetanic stiffening of the whole body, contractions of the extremities, and, finally, deep coma.

Partial sclerosis gives usually no evidence during life of its presence, unless it occupies a large centre, when it may give indications of pressure on the brain, especially somnolence, and its form may be more or less influenced by other diseases producing it, as suppurative meningitis, atrophy of the brain, extravasation, \&c.

Hypertrophy of the brain with extensive sclerosis has always a fatal ending, whilst partial sclerosis may not interfere with life. The duration of the disease is various, and cannot be determined with certainty. In one case $I$ was enabled to fix it at seventeen months, in a case where it was congenital.

Causes.-All that we yet know of these is conjectural. In one case, as I have mentioned, the disease was congenital, and the child lived seventeen months. The majority of my cases were about three years old. The disease often attacks a chill 
in the midst of perfect health, and neither in the history nor in any point of the child's condition can an explanation of its origin be found. Frequently the children attacked exhibit ndications of rickets or scrofula, generally in the form of enlarged and suppurating glands. Partial sclerosis occurs with atrophy of the brain, meningeal and cerebral hæmorrhage, and also with meningitis and encephalitis.

Diagnosis.-This can never be made with certainty, and, perhaps, only seldom with probability. In the form and increase of size in the head it does not differ in any way from chronic hydrocephalus, and in both cases the fontanelle is distended. The auscultation of the head gives no assistance in the diagnosis.

Treatment.-After what has been said it must be evident that the treatment can only be symptomatic, and it is, unfortunately, in most instances unavailing.

\section{Hydrocephalus, serous exudation in the Brain and its} Membranes

In the widest meaning of the term hydrocephalus all collections of serous fluid within the cavity of the skull may be included in it. It may be congenital or acquired, acute or chronic, and due to general or local causes. Serum may collect between the arachnoid and dura mater as external hydrocephalus, in the meshes of the pia mater as cedema of the pia mater, in the ventricles as internal hydrocephalus. Of 200 cases of hydrocephalus, I have found 100 to be of the internal variety, 80 to be cedema of the pia mater, 10 to be cases of external hydrocephalus, and 10 cases of simple cerebral œdema.

In the following remarks I shall treat separately of the con: genital and acquired varieties.

\section{(a) Congenital Hydrocephalus}

'This disease is developed during intra-uterine life, and results either in premature death of the fotus, or in protracted labour when the children are born at the full time. Sometimes the collection of fluid previous to birth is not great, but increases rapidly after birth.

Anatomy.-Congenital hydrocephalus is seldom of the external variety ; but, as a rule, the effusion is found in the ventricles, 
and very exceptionally both forms are met with in the same case. A very marked disproportion is at once visible between the enormously increased cranium and the suall face, in some extreme cases the former measuring from twenty-four to twentyeight inches, and in one child, only nine months old, I found it to measure as much as thirty-seven inches, the length of the body being only twenty-six and a half inches; the height of the head was seven and a half. The head is generally symmetrical in form, though it may exhibit asymmetry in various degrees, induced by unequal ossification of the skull, or by synostosis of some single suture, by continuous lying on one side, and, per'haps rarely; by unilateral hydrocephalus. Congenital hydrocephalus may also exist with very slight increase in size of the skull or even with an abnormal smallness of it (microcephalism). As an instance, I have seen a congenital internal hydrocephalus of considerable size with only ten and a quarter inches of skull circumference. The integuments are found very tensely stretched over the enlarged skull and beset with sparse hair, and in extreme cases distinct fluctuation may be made out. The plates of bone are either uniformly thinned and easily indented, or at some places, especially towards the occiput, interrupted by spaces, which are bridged over by membranous expansions scattered with islets of bone. The fontanelles, especially the anterior, may be several inches wide, contracting into the sutures, which may be still open to the extent of several lines. The plates of the occiput, parietal and frontal bones, are all arched strongly outwards, and when rickets exists simultaneously roundish, dark red periosteal swellings may be found in the latter two bones. In retarded ossification of the skull there are sometimes found encephalic protuberances in the regions of the foritanelles and sutures.

When the skull has been opened the brain protrudes en masse, like a large bag, the convolutions much flattened, the gyri and sulci being levelled, the ventricles distended to sac-like cavities, with the walls thinned so as to be only one or two lines thick, and very easily torn; the rupture is generally perforated or exists only as a bulkhead framework. I have once seen the foramen of Monro so wide that a hen's egg might have been passed through it. The optic thalamus and corpus striatum are flattened, the crura cerebri separated, and the cerebellum 
flattened. The cut surfaces of the central ganglia and the cerebellum, when the disease has been of long duration, are lardaceous, dense, and homogeneous, with indistinct separation of the grey and white substances; and there are also found in the white matter spots of firm, hard tissue, exhibiting on microscopic examination, fat-granules (Lambl).

The quantity of fluid contained in the ventricles varies from four or six ounces to six or seven pounds, and may be clear and transparent or slightly turbid, of alkaline reaction, and albuminous. According to Schmidt, potassium salts and phosphates are those chiefly present.

In external hydrocephalus the pressure exerts its influence from above downwards, and the brain thereby undergoes various alterations; the fossæ of the skull are more or less excavated, and, in extreme cases, form one common deep cavity, from which the roofs of the orbits are more or less indented. In one case I saw spina bifida, clubfoot, and cleft palate coincident with congenital hydrocephalus.

Causes.-Arrest of the development of the brain and intrauterine ependymitis are causes of congenital hydrocephalus which are partly ascertained and partly conjectural. There can be no doubt that the disease is frequently hereditary, though what influence marriage of relations, syphilis, advanced age of the parents, drunkenness, \&c., can have on its production can only be asserted.

\section{(b.) Acquired Hydrocephalus}

This may occur either in the external or internal form, or as œdema of the pia mater or of the brain. It may be acute, subacute, or chronic, though in the result we distinguish only acute and chronic hydrocephalus, the former occurring most commonly as an adjunct of tubercular meningitis, though it may have an independent existence.

Anatomy.-Acquired internal hydrocephalus shews all those changes already referred to as being produced in the congenital affection, with this difference, that it does not reach the same extreme extent, especially when it is developed in a skull already closed, or nearly so. The ventricles may become dilated to an extent varying from three to six times their normal size, the 
cornua being generally rounded, and the quantity of fluid varying from four to eight ounces. In the acute form the ependyma is hyperæmic, frequently loosened, faint-looking, or pultaceous and softened; the fornix, septum, and surface of the corpora quadrigemina are also frequently similarly affected. In chronic hydrocephalus the ependyma is thickened, firm, and occasionally scattered with connective tissue granulations; the choroid plexuses are empty, pale and often occupied by small cysts; the brain substance is anæmic, tough, soft and œdema. tous. In one case of chronic hydrocephalus I have seen the sutures, which had previously been at least in contact, opened to the, extent of four lines.

Edema of the brain is characterised either as a mere moistening through of the brain-mass, or as an abundant collection of serum in it. In the first case the cut surfaces are strongly glistening, in the latter the fluid exudes in the form of drops. The brain is either very markedly anæmic and more or less softened, or bears signs of hyperæmia. Cerebral œdema is frequently combined with œdema of the pia mater and dropsy of the brain cavities.

Edema of the pia mater concerns, for the most part, the convexity of the brain, and produces greater or less collection of serum, sometimes clear, sometimes reddish coloured, on the surface of the brain and between its separate convolutions. This is very often associated with an increase of the contents of the ventricles.

Causes.-Of eighty cases of acquired hydrocephalus

\begin{tabular}{|c|c|c|c|}
\hline \multicolumn{4}{|c|}{22 children were under 2 years old ; } \\
\hline 17 & $"$ & 3 & " \\
\hline 7 & ,• & 4 & " \\
\hline 4 & , & 5 & , \\
\hline 2 & " & 6 & , \\
\hline 2 & , & 7 & ” \\
\hline 1 & " & 8 & " \\
\hline 1 & ", & 9 & " \\
\hline
\end{tabular}

Collectively, the causes of hydrocephalus may be, according to Bamberger, comprehended in two groups:-I. Brain disease, and abnormal conditions of the circulation in the brain. II. 
Altered (serous) condition of the blood, either independent, or produced by disease in another part. All diseases which conduce to one or both of these states may consequently produce the condition of dropsy of the ventricles, œdema of the pia mater or of the brain. As such xtiologic factors are to be taken tumours in the brain, swellings in the neck, exhausting diseases, especially chronic intestinal affections, rickets, kidney diseases, hooping-cough, heart disease, laryngeal croup, tuberculosis, scrofula, broncho-pneumonia, and more rarely the acute exanthemata. Acute hydrocephalus not unfrequently precedes the chronic.

Symptoms and course.-The various lesions which oocur in the course of hydrocephalus are partly of a mechanical nature, due to the pressure of the increased serum and partly to the altered nutrition and activity of the cerebral elements. When the symptoms of acute hydrocephalus have passed off in any case, it may be fully expected that chronic hydrocephalus will ensue. The variation in size, in form and shape, is very considerable in degree, accordingly as the hydrocephalus is congenital or acquired, and they appear with more or less rapidity as the skull is still enclosed or the sutures ossified. The enlargement occurs most readily in congenital hydrocephalus. the disproportionately large head, which the child seldom can carry upright; the little pinched, withered, and aged face; the markedly dilated veins in the forehead and temples; the protruding, half-closed cyes, and the arrested development of the rest of the organism, all contribute to give an appearance to such children which is quite peculiar to them. These conditions are found less prominent in acquired hydrocephalus, especially in the instance of the nutrition and development of the body, which is often well advanced, and quite in accordance with the age of the child.

Mental activity is the cerebral function which is the first to suffer, and it may be interfered with to a degree that will amount to absolute idiocy, so that the child may he entirely incapable of education, or may be capable only to a very limited extent. Occasionally, even when there has been a marked degree of hydrocephalus, I have found the mental powers unaffected. The organs of will are often uninjured, but generally are more or less weakened, especially in children who have 
been rendered partially or entirely blind. Strabismus and nystagmus are frequently met with.

The senses of hearing and taste suffer sooner or later in the course of the disease, from the pressure of the accumulated serum. The sensibility of the skin is generally diminished, though occasionally hyperæsthesia or even a sense of pain on touch may be observed. Motor lesions are generally indicated by the inability of the child to sit or stand, or from its ability to walk being developed very late or never at all. Partial or general convulsions, muscular contractions, paralyses, automatic movements of one or both upper extremities of great variety, appear at intervals. Pain in the head, especially when the child is raised upright, vomiting, great restlessness, continuous crying, biting and gnashing of the teeth, and profuse flow of saliva are all very constant symptoms. The digestion is usually pretty good, many children exhibiting extraordinary gluttony without gaining any increase of weight. Constipation is a pretty general condition in hydrocephalus. In the course of the chronic hydrocephalus there often occur remarkable exacerbations of an acute kind without apparent cause, and which, in all probability, make each time an addition to the exudation. Symptoms of severe cerebral irritation, as vomiting, great restlessness, epileptiform seizures, mania, \&c., often make a transient appearance, but under such attacks death may occur. There is considerable difference in the duration of the disease in different cases, though it may be taken as the rule that the affected infant will not reach childhood, but that it will succumb during early life either to the cerebral affection, to the troubles of teething, or to one or other of the many complicating discases, as pneumonia, bronchitis, intestinal catarrh, dysentery, \&c. In some rare cases adult life is reached, such an instance having come under my own observation in a person thirty-two years of age, the diameter of whose skull is still so great that he has much difficulty in providing himself with suitable hats, but whose mind is so well developed that he is employed as a copyist. Gall mentions a case of hydrocephalus which had reached the age of fifty-four years. If arrest of the disease, or recovery, should take place, it is accomplished by an increase in bulk of the bones, these sometimes reaching a thickness of an inch, and by an accommodation on the part of the brain. 
Serous apoplexy, or acute cerebral œdema, always occurs suddenly and is rapidly fatal, sometimes in a few minutes. It is met with in children who appear to be or really are wellnourished, though on more minute examination, indications of rickets may be found, or even a certain kind of latent ventricular dropsy. In all the cases that have come under my notice I have found some such starting-point, and I cannot agree with the authors who assert that it occurs in children who have been previously in perfect health. The majority of the cases occur during the first and second years of life, and death usually takes place with such suddenness, that the amazed parents can liardly regard it as real; for the child, previously looking in the best of health, whilst even smiling, may be seized by a slight convulsion and be in a few minutes a corpse.

Diagnosis.-For this we may refer to what has been said of tubercular meningitis. Idiopathic acute hydrocephalus, an affection of great rarity, has a train of symptoms very similar to that of acute cerebral œdema, and it is not easily possible to diagnose between them.

Edema of the pia mater, often complicated by a slight degree of ventricular dropsy, gives rise during life to a train of symptoms closely resembling those of cerebral anæmia, and a differential diagnosis can only be made by an accurate estimation of the causal factors. Extreme cases of chronic hydrocephalus, especially when congenital, offer no diagnostic difficulties, though less well-marked cases may be confused with cases of rickety affection of the skull. In such cases, however, the presence or absence of the periosteal swellings on the frontal and parietal prominences, and indications elsewhere in the skeleton of a rickety condition, also the presence or absence of the severe symptorns of cerebral depression belonging to hydrocephalus, will assist materially in the determination of the nature of the case. Cranial auscultation, which has been recommended by many authors to assist in the diagnosis of such cases, has proved of no service in my experience.

It must be held in mind that rickets and hydrocephalus frequently coexist. True hypertrophy of the brain occurs so rarely in infants that there can hardly be any diagnostic doubt on its account. On the other hand, tumours of the brain occur with sufficient frequency to lead to difficulty. 
Prognosis.-For the majority of the cases this is unfavorable, or at least doubtful, whether the affection be congenital or acquired, acute or chronic. Acute hydrocephalus, as has already been noticed, is almost always fatal. Partial absorption of the secreted fluid seems to be possible, for it has been observed during life, and it seems to be more likely the further advanced is the ossification of the skull. Frequently recurring signs of brain irritation may always be looked upon as unfavorable symptoms.

Treatment.-Such treatment as was discussed for meningitis may be recommended for hydrocephalus and œdema of the pia mater, though there seems to be none that has any certain result. By means of judicious prophylactic treatment the occurrence of secondary hydrocephalus may be warded off in cases where its possibility may be suspected, as in long-continuing intestinal catarrh, chronic broncho-pneumonia, rickets, cerebral anæmia and hyperæmia. Chronic hydrocephalus gives, unfortunately, no satisfactory results from treatment, for neither the external application of deobstruent ointments, nor the internal administration of diuretics, nor diaphoretics, nor compression by plaster, nor puncture and evacuation of the collected fluid, have resulted in a cure, though all have had frequent, trials. They all really amount to experimental torture, and are being more and more completely abandoned.

\section{Tumours of the Brain and its Membranes}

Among the tumours which appear in the infantile brain we may take tubercle as the most frequently observed; only exceptionally are carcinomata, sarcomata, glyomata, syphilomata, and parasitic tumours (cysticerci) met with.

Anatomy.-Tubercle of the brain shows relatively, in its number, size, its seat and behaviour, manifold diversities. In ninety-four cases observed by myself, and confirmed by postmortem, I found the following:

Brain tubercle occurs sometimes as a single tumour, sometimes simultaneously in many centres; the smaller the tumours, the greater, as a rule, are their numbers; their size varies from that of a pea to a hen's egg, or even a man's fist; the seat of the tubercle is by preference the grey substance, seldomer the white; 
more frequently the cerebrum than the cerebellum, sometimes in both simultaneously, and exceptionally in the medulla oblongata. The surrounding brain substance may be wholly unaffected, or it may be anæmic, hyperæmic, sometimes softened, or small extravasations may be found in the immediate neighbourhood. Internal hydrocephalus and œdema of the pia mater form frequent complications, though they may also be absent. In rare cases cerebral tuberculosis occurs as an independent lesion, there being no indication of cheesy or tubercular deposit anywhere else in the body, though, as a rule, there is associated with it tubercular disease of the glands or of the lungs, \&c.

Tubercular meningitis is oftener than not a complication of cerebral tubercular tumours. The tubercular centres are met with as yellow, cheesy, more or less dry masses; more rarely they occur as grey, hardish knots, surrounded by a reddish layer. Papescent softening in the centre of the tumour is occasionally found, and Rilliet, Berthez and West have met with calcareous degeneration.

Carcinomatous tumours are rarely found. They may have an origin in the brain or meninges, or they may appear as secondary growths advancing inwards from the skull. In the former case the tumour may break through the bones of the cranium, as I have seen in one case. A very frequent origin of secondary cancer of the brain is its recurrence after extirpation of the eyeball for cancerous degeneration, and in such cases I have seen the secondary cancer originate simultaneously in several centres.

Sarcoma is also rarely met with, but when it does occur it forms smooth, rounded, firm tumours, sometimes of large size. One such I have met with, the size of a hen's egg, situated in the optic thalamus of a girl four years old.

Of parasitic tumours $I$ have seen the cysticercus cellulosa three times in the brains of children, and once the echinococcus.

Symptoms and Course.-We cannot describe any symptomcomplex which would include all tumours, for, on account of their slow and gradual development, their difference in size, number and seat, and the varieties of their relations to the surrounding structures, it is quite evident that each case will have its own peculiarities. 
Numerous cases have shown that tumours of large size may grow and be in existence for years, even till near their fatal issue, without giving rise to any suffering that might indicate their presence. 'This I have seen in three cases of large tubercular tumours, situated very superficially in the lobes of the cerebrum. Still, out of the great number of varying and often contradictory symptoms the following may be selected as likely to be of use in the diagnosis of cerebral tumours :

Unilatcral convulsions, paralysis, and muscular contractions which occur periodically or are persistent in the same nervetrunks, indicate with great probability, in children, the existence of some centre of disease in the hemisphere opposite to that of the affected side, and when there are other indications of tubercle or scrofula there is a great likelihood of a tubercular tumour.

Epileptiform convulsions occurring at first with long intervals, but afterwards more frequently, and always in the same group of muscles on the same side or, if bilateral, always with the same characters, may also give rise to the suspicion of a cerebral tumour.

Tumours of the hemispheres may remain completely latent, or may produce only occasional obstinate headache, giddiness, vomiting, and afterwards hemiplegia, convulsions, or coma.

Tumours at the base of the brain generally give early indications of pressure, as interference with and weakening of sight, amaurosis, strabismus, ptosis of one or both upper eyelids, vomiting, pain in the head, epileptiform convulsions, and paralysis of single or several muscle groups. In tumours of the pons, medulla oblongata, and cerebellum, I have seen intermitting or continuous pain referred to the occiput and passing down the neck, straining of the head backwards, and, further, which are of especial importance, unsteady gait, disturbance of the power of co-ordinating movements, stumbling, trembling, or chronicquivering of the muscles, disappearing when the patient is lying quiet, but becoming very pronounced when he attempts to walk. In tumours of the pons I have seen cpileptiform convulsions, which increased in severity, and in one case there were severe and frequent convulsions of the upper half of the body.

Tubercle of the corpora quadrigemina and peduncles, after 
a train of symptoms not very constant, generally produces paralysis of the oculo-motor nerve, indicated by ptosis, \&c.

In a case of sarcomatous tumour, the size of a hen's egg, in the right optic thalamus, observed by myself in a girl four years old, there was no other lesion of the eyesight than dilated pupils; on the other hand, there was great disquiet, frequent outcrying, general muscular weakness, back-bent head, contractions of the upper extremities, irregular respiration, and irregular but retarded pulse; later on stupor and slight convulsions occurred. The ventricle was found much distended, and was almost completely occupied by the tumour. The left optic thalamus was slightly developed and somewhat flattened, and the meninges at the base corresponding to the middle bone were opaque.

The general functions of the body may remain a long time uninjured by the presence of a cerebral tumour, the digestion and rest being uninterfered with, and the principal functions remaining healthy until the resulting lesions make their appearance. The pulse then may be retarded and the breathing become irregular, and micturition may either be impeded or the urine may be passed involuntarily. The duration of cerebral tumours can seldom be ascertained with certainty, on account of their prolonged latency, and, therefore, it may be guessed at either by weeks or years. Death results, as a rule, from some secondary process in the brain and its membranes, as acute or chronic hydrocephalus, hæmorrhage, encephalitis, meningitis, or acute miliary tuberculosis.

Causes. - The most frequent causes of brain tumours in infancy are scrofula and tuberculosis, and we find in children so affected, together with the tumour in the brain, indications of these conditions in the neck, lungs, \&c., and in these we have valuable aids to the diagnosis of the case. We see gummatous tumours in children due to syphilis very rarely; and, if we add tumours of a parasitic origin, we close the list of those whose origin is known to us; all the others are ætiologically obscure, though sometimes an exciting cause, as a blow, may be traced. Tubercular tumours occur more frequently in boys than in girls, and are met with from the first year of life up to puberty, and, perhaps, may sometimes be congenital, though there is as yet no good evidence on this point. 
Progniosis.-This is unfavorable, as may appear from the fact that there have been 94 cases of tubercular tumour out of 100 cerebral tumours which have come under my notice. The fatal result is often sudden, as has already been noticed.

Treatment.-This can be directed against the general scrofulous or tubercular condition of the patient, or may be symptomatic and palliative, with a view to mitigate some of the secondary symptoms induced by the tumour. In severe headache, cold applications, ice on the head, or derivation by means of sinapisms on the neck, back, and lower extremities, opium or subcutaneous injection of morphia may be employed; in epileptiform convulsions oxide of zinc, bromide of potassium, cold packing and rubbing of the whole body may be tried. We also always ensure daily alvine eyacuations, and supply the patient with easily digested and strong nourishment. Mental occupation or exertion-where these may still be possible-are to be carefully avoided. If there is a probability of a syphilitic origin for the tumour, an antisyphilitic course of treatment ought to be tried.

\section{Mental Diseases}

Idiocy, in its various degrees, is frequently seen in infancy, but other mental affections are rare exceptions, the reason for this being evident in the fact that the existing and predisposing causes which conduce to mental diseases in adult life are still in abeyance during infancy and childhood. The stormy passions of grief and anger, the disappointments and failure of hopes cherished, or remorse for past conduct, have but little hold on the elastic spirits and lightly beating heart of the child; whilst the bodily causes of mental disease, as disease of the brain and other organs, are not found in their severe forms in children, or they result in death before the mental affections become developed.

In spite of this, however, there come, every now and then, before the busy practitioner, well-marked instances of mental disease in children. 


\section{Mental Excitement (Mania)}

This may be due to some acute or chronic irritation of the brain, and may be transient, leaving behind it no permanent lesion, or it may be progressive, and finally remain as idiocy throughout adult life. I have seen acute mania in children between the sixth and thirteenth year of life as the prodromal symptom of variola, also after insolation, and as a sequela of typhoid fever. In these cases the cause was the hyperæmia, simple or toxic, and in the case of typhoid hydrocephalus internus became developed.

I have seen a case of periodic mania, in a boy six years old. which finally degenerated into idiocy. Previous to the commencement of the attack he was quite healthy, and I hazarded a conjecture that the disease was due to a cerebral tumour with consecutive hydrocephalus. The mother of the child was an extremely delicate and nervous woman. The mania occurred in such severe paroxysms that we had to employ restraint and large doses of opium, and a number of adults were always necessary to subdue him.

The disease sometimes takes the form of a frenzy, as in one case where a boy, twelve years ago, was possessed with a fixed and frantic idea that his father wished to murder him. The boy was well grown and nourished, but his face bore a constant anxious and unquiet look, his head felt very hot, his sleep was very slight, the pulse slow and with marked intermittence. As soon as his father appeared the restlessness was markedly increased, and he made eager efforts to escape either by the door, window, or fireplace. He was removed to the house of a relative, but he did not improve there, and begged to be taken home again.

\section{Mental Depression (Melancholia)}

This occurs in children as slight melancholy or hypochondriasis, and may remain in existence till adult life, if not looked after in time and suitably treated. Cases have also been observed where children otherwise healthy, though often weakly and anæmic, have imagined themselves suffering from certain diseases of which repeated examinations have never 
revealed the real existence. This has frequently its origin in the bad example of the parents, especially of an hysterical mother; in perverted education, as is now-a-days encouraged by the spread of immoral works of over-strained fiction; in premature love dalliances, and from a dread of early death. I have had under my care a boy, six years old, whose only playmate was his sister. She died of tubercular meningitis, and he soon fell into a melancholy from which neither persuasion nor amusement could rouse him. The thought that he too must soon die of the same disease tormented him continually, and he lost both appetite and sleep. This condition lasted about two years, and then he gradually lost all trace of it.

Slighter cases of melancholy are often noticed in girls at puberty, due to the physiological change and its concurrences.

\section{Mental Weakness (Imbecility)}

Under this head is to be classed the large majority of cases of disease of the mind in children, forming the various kinds and degrees of idiocy. Endemic idiocy, or cretinism, occurs chiefly in mountainous districts, and can here be only incidentally alluded to, for we have to deal more with the sporadic form. During twelve years I have met with 14.0 cases of idiocy, many of which have been of such slight degree that they were more or less capable of instruction, whilst others have been so bad as to be incapable of anything. The condition is generally congenital, and is accompanied usually by abnormalities of cranial development, as microcephalism, rudimentary development, or hydrocephalus; more rarely it is acquired during the early years of life. In some cases of idiocy absence of part of the brain is found; also, more or less extensive sclerosis. The various types of idiocy are grouped, according to Griesinger, as follows:

I. Children with no malformation, generally of kindly disposition, and, as a rule, tending to microcephalism. The mental development may be of very various degrees. The children are generally lively, though often in an apathetic condition, moving in a lifeless, automatic manner, and they frequently have a marked want of power in the lower limbs.

II. Children who are simply instances of arrest of bodily or 
mental growth, the arrest seeming to have taken place at various ages, and the characteristics of the age at which it has occurred being permanently retained.

III. Cases of cretinism, due to synostosis of the base, with extremely deficient intellects.

IV. The aztec type, marked by microcephalism, but also wellproportioned, and correspondingly slender development of the body. They are very lively, all their motions being well coordinated, sprightly, easily excited, inquisitive, capricious, and very weak-minded. Gratiolet found the skull very small, the bones thick, and the ossification of the sutures well advanced, but the bones at the base only slightly ossified, almost wholly cartilaginous, the petrous and ethmoid bones being somewhat larger than normal, and the fossa for the cerebellum of very large size. The convolutions of the brain have been found smaller than those of the ourang or chimpanzee; the cord, medulla, and cerebellum disproportionately large, and so, also, the organs of sense and their nerves.

V. A few idiots who approach, in their appearance, habits, and behaviour, certain of the lower animals, as apes and swine.

It would be impossible to point out all the various lesions that are met with in idiotic children. As a rule, the wellknown idiotic expression of the face is marked, and there is a delay in, or complete absence of, the acquisition of the powers of speaking and walking. There is an obtuseness of some of the senses, as deafness, and very generally a deficiency of cleanly habits. Epileptic seizures are common, and sometimes there is some form of paralysis. Parents are often completely blind to the mental defects of their idiotic children, seizing with the fondest hope on the faintest gleam of intelligence, and seeking the assistance of the specialist for the deafness or the delayed acquisition of speech, in the hope that such aid will afford relief for the unfortunate condition.

The duration of life in idiots and cretins depends essentially on the severity of the nerve lesion, which forms the basis of the condition, and it is noteworthy that idiots seem to have much less power of withstanding the influences of disease than other children. 
Treatment.-The removal of idiotic children from their homes, . and their being placed in a special institution, is an essential element of success; for in this way, and by the encouragement of the special capabilities developed in each case really wonderful results may be obtained.

For this purpose the number of idiot asylums ought to be increased, to which at least the worst cases ought to be removed away from the care of their relations.

\section{Congenital Arrest of Development}

These cases have already been alluded to when speaking of hydrocephalus, and to this class belong the various defects of cerebral development seen in acephalism, when it is complete, in cyclopia and other monstrosities where it is partial. All these have more of an anatomical interest than of clinical importance.

\section{A.-Microcephalism}

This results from two processes, either there being an arrest of nutrition and growth of the brain and skull, both remaining small without synostosis of the latter; or there may be alterations in the bones producing premature synostosis, resulting in diminution of the brain as an effect. Vogt indicates microcephalism as the condition where the child is born with an absolutely small head, and where synostosis has nothing to do with the arrest, the explanation being solely an arrest of development dating from about the tenth week of life, and affecting chiefly the part of the brain which has to do with the mental powers. From his observations, the brain of the microcephal exhibits the great typical convolutions, but in many the convolutions are so indistinct that the anterior lobes seem entirely destitute of them, or to exhibit them only as indistinct furrows. It seems to me, however, that Vogt's view is too limited and partial, and it undoubtedly includes cases where the microcephalism has arisen in synostosis of the skull (Virchow).

The symptoms observed are a marked and disproportionate size of the skull, which may also be variously malformed, the forehead is very shallow, the expression of the face more or less idiotic, vacant, or even animal; the mental powers are of a low 
order, and typical microcephalæ are seldom capable of any education. According to Vogt, microcephalæ have never been found capable of speech who had a brain volume of only about twenty-eight cubic inches. Besides these mental lesions there occur disturbances of the motor powers, especially of the lower extremities, contractions, and convulsions. Thus I have seen severe convulsions alternating in a microcephalic child, eight months old, occur each time before the passage of a new tnoth, and they were always followed for two or three days by other symptoms of cerebral irritation. Microcephalism to a small extent does not preclude the possibility of a certain amount of instruction.

We are yet ignorant of the origin of this arrest of development, and, according to Vogt, hereditary transmission would seem to have little to do with it, for the majority of cases are the children of healthy parents, and they are often born between children that are normally developed. In some families there seems a certain tendency to the production of microcephalism, but there seems no better reason to refer its origin to the parents than exists in the case of other deformities. ${ }^{1}$

\section{3.-Encephalocele and Hydrocephalocele-Hernia of the Brain}

Simple encephalocele or cerebral hernia occurs when a part of the cerebrum or cerebellum protrudes through a cleft of the bones of the skull, and when together with this protrusion there exists a greater or less collection of serum. it is termed a hydroencephalocele.

These conditions are usually congenital, though an encephalocele may be the result of wounds or necrosis of the bones of the skull, an instance of the latter cause having come under my own notice in the case of a child in whom the hernia was as large as an apple. The congenital hernia most usually occurs in the hollow of the occipital bone, in the nasal part of the frontal bone, though it may also be met with in the smaller sutures. The size varies from that of a pea to that of a child's head, according to the position of the prolapse and the

\footnotetext{
' ' Jahresbericht des Vereins Pract. Aertze in Prag,' 1869-70.
} 
quantity of serum present. In shape the hernia may be pointed or flattened. The tumour is covered by healthy skin, though it is often thinned and devoid of hair, usually pulsates, and is sensibly influenced by strong respiration, especially by cries and coughs, and it may be more or less reduced by pressure, though this must be attempted with the greatest care, as manipulation may readily induce serious symptoms, as vomiting, convulsions, unconsciousness, tetanic contractions, or paralysis.

Large extrusions of the brain may be mistaken for cephalohæmatoma; but the seat, besides the evident pulsation and the possibility of lessening the tumour by compression, together with the resulting brain accidents, will soon enable the lesion to be recognised. Smaller brain extrusions may be mistaken for erectile tumours, and $\bullet$ in such cases repeated careful examinations ought to be made before any operative proceedings are undertaken.

The prognosis must always be unfavorable, even though some few cases of spontaneous cure or successful operative interference would seem to show that sometimes a fortunate result may be obtained, yet the general result is scarcely affected by these rare cases.

'These cerebral herniæ seldom remain of their early size, but usually advance with the growth of the child, and induce serious disturbance of its mental powers when of large size, being usually accompanied by a mental condition more or less approaching idiocy.

Treatment.-When the hernia is small it may, perhaps, be proper to attempt its reposition, but any effort of this kind must be desisted from should there occur any cerebral symptoms. In general, all that can be done is to protect the hernia from injury by a concave metal plate. Should there be a collection of serum, repeated acupuncture, or the evacuation of the fluid by an exploring needle, may sometimes be of service, though recovery has occasionally taken place without any such interference.

Vogel mentions a preparation in the anatomical collection at Munich where, in the occiput of an adult, an aperture the size of a groschen, with the edges rounded, is to be seen, from which, during life, a cerebral hernia extended. Operations on such cases are seldom undertaken, on account of their great peril. I have seen a hernia of the nasal part of the frontal bone where 
an operation was fatal in thirty-six hours from suppurative meningitis.

\section{c.-Cephalohæmatoma, Thrombus Neonatorum}

Under the term cephalohæmatoma are grouped three varietics of external hæmorrhage, which vary as to their seat. The cephalohæmatoma proper (C. externum) consists of an extravasation between the pericranium and the bones of the skull; in C. spurium, or subaponeuroticum, the blood is found above and below the galea aponeurotica; and, lastly, in C. internum it is situated on the inner surface of the roof of the skull between the bones and the dura mater. C. externum and internum are sometimes found coexistent.

Cephalohæmatoma externum is covered by normal skin, is a flattish round tumour, of a size from a pigeon's egg to an apple, more or less elastic, fluctuating, and generally painless, and it is always noticed at birth or soon after. It is surrounded and limited by a perceptible bony ring. Its seat is usually on one or both parietal bones, and certainly more frequently on the right than on the left side, now and then occurring on both. Ritter has observed, in seventy children, that it occurred on the right side forty-one times, on the left twenty-two times, and seven times on both parietal bones. If the swelling be left alone it disappears sooner or later, and the elevated skin becomes flattened, though in some rare instances the contents undergo suppurative alteration, and the tumour evacuates itself as an abscess, as I have seen in two cases, or it may lead to necrosis of the bone immediately underlying it. As it disappears the limitary ring feels more distinct and rougher.

The causes of this blood tumour have not yet been satisfactorily explained, for it is becoming more and more apparent that the view of its traumatic origin during birth is not always sufficient; for Nägele, Meissner and others, have found it in children who have been born in the position of breech presentation. According to Langenbeck and Ritter the condition is due in the first instance to an incomplete development and formation of the external table, and this opinion is supported by the examination of sections; and in this is found also an explanation of the curious rings of bone previously mentioned. 
The opinion which has been expressed that there is some special thinness and fragility of the vessels of the bone has not yet met with any anatomical proof.

C. externum, when it is present alone, as a rule, results in recovery by absorption; if it is complicated by $\mathbf{C}$. internum it is almost always fatal. The hæmatoma subaponeuroticum, with the effusion of blood between the galea aponeurotica and periosteum occurs doubtless as a result of the birth-act, and is met with as a diffuse tumour, not fluctuating, and surrounded by bony ring, with greenish or brownish-yellow colouring of the skin of the head, and it disappears as a rule by absorption. C. interum has been already discussed under Pachymeningitis, and I may refer to that chapter concerning it.

Diagnosis.-Cephalqhæmatoma differs from cerebral hernia in its seat, in the absence of pulsation, and the fact that it is not increased when the child cries, and that it cannot be replaced. The caput succedaneum, the ordinary scalp tumour, with which the cephalohæmatoma may possibly be confused, is not so sharply defined, may be developed in any part of the head, but shows a soft doughy consistence, produced by a simple œdema of the skin of the head. It is still less possible to mistake it for an erectile tumour, for the situation and character of that is sufficient to preclude such a mistake.

Treatment.-The treatment of cephalohæmatoma, fortunately for the affected children, has become in course of time a very simple one. Patient waiting brings sooner or later, according as the tumour is greater or smaller, perfect dispersion and absorption. Tincture of iodine, iodine ointments, aromatic spirits and red wineare still valued as local remedies, whilst other authors recommend a continuous cautious compression by means of metal plates, or the painting on of collodion, as favouring absorption. Opening the tumour by means of puncture or a cross cut, as $I$ have seen several times practised in former years, and which still is warmly advocated by many, does not result in recovery so quickly as one would expect. If suppuration occurs, poulticing and the early opening of the abscess is required. 


\section{Diseases of the Spinal Cord and its Membranes}

Unfortunately post-mortem examination of the spinal cord is seldom practised in children, and therefore we know very little of those of its diseases which have any pathological appearances. They are said to be much less common than they are in later life.

\section{I-Hyperemia of the Spinat. Marrow and its Membranes}

This may be either a primary or secondary condition, probably being only rarely primary. The anatomical appearances consist essentially in a greater vascularity of the organ; the meninges are permeated by distended or even varicosely enlarged vessels; the spinal marrow is rose coloured on fresh section, and scattered by numerous points from which blood flows when the cut is made. These alterations may occur throughout the entire length of the cord, or be confined to certain sections of it.

A slight degree of hyperæmia may have no perceptible clinical symptoms as its results, while if more severe, and especially if affecting an extensive tract of the cord, symptoms of pressure may occur, which may be transient, or amount to weakness and difficulty in motion, or even to paralysis. The train of symptoms may be complicated by simultaneous disease of the brain.

The causes are as yet but little known. I have found it after tetanus, chorea, rheumatism, acute exanthemata, caries and wounds of the vertebræ. In new-born children a wellmarked hyperæmia is often seen, but no explanation has yet been offered of it.

The treatment can at best be only symptomatic, even when the diagnosis can be made with probability or certainty, and must chiefly refer to the exciting causes. Sometimes the hyperæmia results in hæmorrhage, though this is not commonly noticed, and then the train of symptoms becomes still more obscure.

This hæmorrhage occurs most commonly in the upper half of 
the spinal canal, though if it occur from the meninges the extravasation may occupy the whole length of the cavity. This latter condition may often be seen simultaneously with hæmorrhage into the cranial cavity, and is then generally the result of injury during birth. Hæmorrhage in the substance of the cord almost always is met with in the capillary form. I have seen capillary extravasation to a more or less limited extent in fatal chorea and tetanus.

\section{Meningitis spinalis-Inflammation of the Membranes of the Spinal Cord}

Not often occurring as a sporadic disease in infancy, it appears as meningitis cerebro-spinalis epidemica sometimes in great abundance. I shall mention only the first variety here.

Anatomy.-The inflammation attacks either the dura mater, and is characterised by strong injection of the latter, also of the surrounding cellular tissue, and, further, by an accumulating exudation on the free surface; or it attacks the pia mater, which becomes more or less injected, and is scattered with serous fibrinous material or purulent exudation. In many cases a large quantity of pus and serum is found in the arachnoid sac. The substance of the cord is often softened, especially on the surface; in other cases I have found it abundantly covered by exudation, strikingly prominent, uniformly hard, firm and anæmic. Only seldom will this exudation be found altered to a dry cheesy consistence, leading to compression and atrophy of the spinal marrow.

Symptoms and course.-In young children the diagnosis is difficult, for all that may be noticed will be a slight tetanic stiffening of the limbs, succeeded by irregular jerkings, a small thread-like pulse, scanty and superficial respirations, and a painful whimpering, especially when they are moved.

Older children complain according as the inflammation has its seat in the upper or lower segments of the spinal marrow, or in its whole extent, of stinging pain in the attacked region, which will be increased by movements. Stiffness of the limbs, of the nuuscles in the neck, trismus, great sensitiveness, tonic muscle-cramp of the trunk and of the 
extremitics, amounting even to perfect tetanus, involuntary passage of urine and fæces, suspended respiration, small quick pulse, and a somewhat increased temperature, with subsequent cyanosis, paresis, and actual paralysis of the upper and lower extremities, form the symptom-complex. This may more or less be influenced according to the seat and extent of the inflammation, and by the simultaneous presence of meningitis. Spinal meningitis takes in children generally a rapid course, and its whole extent may occupy not more than two or three days, especially in the newborn, resulting in abundant purulent exudation; in more advanced children it may last for two or three weeks. When the disease can be recognised, an unfavorable prognosis may certainly be given.

Etiology.-Spinal meningitis frequently arises from caries of the vertebræ or from injury. In newborn children it is generally the expression of pyæmia. I have found meningitis with purulent exudation, and also with an effusion of fibrinous serum following acute articular rheumatism, and after severe irritation of the cord.

Treatment.-Local bleeding over the region of the spinal cord, and even general bloodletting, are frequently practised, though I do not think that it has been yet made certain that much benefit has been derived from such treatment. Absorbent ointments of iodine and mercury have been recommended; and for the relief of pain and tetanic spasm of the trunk and extremities, opium may be given by subcutaneous injection, and relief is frequently obtained by the use of hot baths. In the pyæmic form quinine is very commonly used, but whatever the treatment be the chance of recovery is very small. 


\section{Meningitis Cerebrospinalis Epidemica-Cerebro-spinal Meningitis}

I class this affection with diseases of the nervous system, for its nature is not yet known, though it is more than probable that the local affection is only an indication of some acute zymotic disease. It is stated on good authority that this disease was noticed during the sixteenth century; and in our own times it has occurred in more or less extensive epidemics in France, Holland, Germany, Italy, England, and America. I have had no personal experience of the disease, and must, therefore, borrow from the observations of Reinecker, Niemeyer, Lebert, Ziemssen, and Hess.

Anatomy.-The characteristic changes are found in the meninges of the brain and spinal cord, and consist in an exudation, often purulent, sometimes only serous, situated on the thickened and reddened meninges at the base of the skull, extending sometimes from the convexity of the brain into the spinal canal, and over the cortical substance of the cord. Hypostatic pneumonia, pleuritis, suppurative endocarditis, intlammation of joints, together with enlargement of the spleen, acute fatty degeneration of the liver, kidneys, and muscular tissue of the heart, and finally swelling of Peyer's glandular patches often occur, though they are not constant phenomena.

Symptoms and course. - Exceptionally the disease shows isolated premonitory symptoms, as pain in the head and down the course of the spine, stiffness of the muscles of the neck, nausea, and actual vomiting. Generally the disease is ushered in by severe rigors and intense pain in the head, which soon extend over the occiput and down the neck. The child gives indications of these through loud crying and moaning, sometimes it happens further, especially in children of the first year, that consciousness is instantaneously lost, and does not return, and these are the worst cases. The head is usually bent backwards, which together with stiffness of the lower jaw or actual trismus, painful hyperæsthesia, pain in the limbs, transient paresis, and actual paralysis, epileptiform convulsions and a tetanic stiffness of the back and the limbs are associated in the further course of the disease. The temperature of the skin is generally 
increased, especially at the beginning, being often as high as $41^{\circ}$ Cent., but may sink again further on. Roseola, petechiæ, or sudaminal eruptions are often seen on the skin, and facial herpes is also a frequent symptom. The pulse is much quickened, small, often irregular, and towards the end of the disease can hardly be felt. Enlargement of the spleen may be observed, especially in severe cases, though it may also be absent; equally uncertain is the occurrence of albumen in the urine. The action of the bowels is usually regular, the thirst is increased, and the quantity of urine diminished. If recovery occurs, first of all the stiffness of the neck relaxes, the convulsions become less frequent, the painfulness of the limbs disappears, a gentle sleep comes on, the pulse gets stronger, and the child has an inclination to eat. A few cases, especially those first attacked, may have a rapid fatal ending in thirty-six or forty-eight hours, but the average duration of the disease is from eight to twelve days, though its course may extend over several weeks.

Diagnosis.-This may be certainly decided by the meningeal symptoms, the defects of function of the spinal cord, and, further, from the typhoid character of the fever and the epidemic occurrence of the disease.

The prognosis, according to the experience of recent observers, is unfavorable. The mortality averages about 50 or 60 per cent., but may vary with the severity of each epidemic.

The treatment has as yet been little agreed upon, each author differing in his recommendations from others. Antiphlogistic methods of treatment have been advocated by some, such as leeching behind the ears, cupping along the spine, cold applications to the head, repeated cold baths, and derivation from the skin and bowels by vesicants and clysters. Others, as Lebert, assert that such active treatment is not well borne, but that much good may be got by treating symptoms, especially by relieving pain by the use of opiates, also by atropine which has been highly spoken of. When there is fever of a typhoid character present quinine may be used, and Wunderlich has recommended iodide of potassium. When collapse is threatened stimulants of rapid action must be freely administered. 


\section{New Formations in the Spinal Cord and its Membranes}

There are very few cases on record of new formations in the spinal cord, and they may be classed as follows :

a. Hyperplasia of the connective tissue with sclerosis of the nervous substance, which may be either partial and limited to small centres, or extend over large tracts of the cord. I have seen this in a boy aged eight and a half years who died of acute chorea, and in whoin the spinal chord, especially in its upper half, was rounded, plump, very anæmic and of an almost cartilaginous consistence. It was a case of well-marked minor chorea, to which were added, in the last few days of the illness, tetanic symptoms.

$b$. Tubercle is found infiltrated in the dura mater in tubercular caries of the vertebræ, sometimes as large masses, and very exceptionally as miliary tubercles.

In the spinal substance itself tubercle is extremely rare. Eisenschütz ('Jahrbuch für Kinderheilkunde,' 2 Hft., 1870) has a case, in many respects very interesting, of a boy three and a half years old in whom, together with general tuberculosis and small tubercular masses in the brain, there was found, at the lower part of the dorsal section of the spinal cord, a small tubercular tumour the size of a pea. The condition was diagnosed from the insensibility which existed during life as far as the eighth dorsal vertebra, together with tremblings of the muscles, especially when the patient attempted any movements, similar to what occur in paresis agitans.

Tubercle in the region of the spinal cord is, in almost all cases, only an accompaniment of tubercle in other organs, and if there is evidence of the latter in association with spinal symptoms, in many cases the diagnosis may be made with probability.

c. Sarcoma and carcinoma are still more rarely found than either of the above-mentioned conditions. Löschner met with one case of sarcoma in a boy aged four years and five months, where a tumour twelve centimètres broad and sixteen centimètres long extended from the left side of the chest as far as the spinal canal, and almost entirely occupied the latter from the fourth to the tenth dorsal vertebra. The spinal membrancs 
were tightly stretched over it, the marrow itself being pressed to the front and flattened. Paralysis of the lower extremities was here the only symptom which pointed to a spinal lesion. The origin of these new formations is quite unknown.

\section{Spina bifida, Hydro-rhachitis, Hydromeningocele, Hydromyelocele}

Congenital abnormal collections of serum, both in the fotal canal of the spinal marrow and also in the subarachnoidal sac, form the essential conditions of hydrorachitis. In the most marked cases the formation of the spinal marrow is completely arrested (amyelia); in slighter degrees the vertebral canal presents an open channel, the spinal marrow being rudimental; whilst in the least well-marked cases it may be present completely developed. Very seldom it occurs that there is no alteration of the bones of the vertebræ; more frequently several or many of the vertebral arches are incompletely developed, whereby, according to the degree of the defects of the bones, a longer or shorter, narrow or broad cleft remains (spina bifida). This occurs most frequently in the lumbar and sacral regions, more rarely in the dorsal and cervical sections of the vertebral column, is generally simple, seldom double or present at several points. 'Ihrough these chasms sacs are prolapsed, distended with serum, and varying in size from that of a hazel nut to a child's head. They are formed from the dura mater and arachnoid membranes, and always fluctuate more or less distinctly. If the spinal marrow also passes through the gap a hydromeningocele is formed-an occurrence which is analogous to the cerebral hernia. If the termination of the spinal cord is inserted into the wall of the sac a peculiar umbilicoid retraction is perceptible, to which attention was drawn by Virchow. Occasionally the sac is divided into two cavities by the chord stretched in to the form of a thin membrane (Bednar).

Bursting of the sacs may occur in the uterus before or during labour, or later by maceration or inflammation of the external covering. Other malformations, as hydrocephalus, hemicephalism, and club-foot, are often seen along with spina bifida.

Symptoms. - The most important of these consists of the 
presence of the above-described tumour, which has usually a broad base, or is somewhat pear-shaped and continued into the vertebral canal by a narrow neck. It often happens that on manipulation of the tumour the pointed extremity of the rudimentary cord may be felt, and by pressure the tumour may be diminished to a marked extent, though by so doing cerebral and spinal symptoms, such as vomiting, convulsions, contractions of the lower extremities, \&c., may be excited. If the sac is not flattened at birth it soon becomes so, and the skin covering it after a short time excoriates, or may take on erysipelatous inflammation, and death may occur from exhaustion or from the extension of the inflammation to the membranes of the cord or to the spinal substance itself. In exceptional cases the normal skin remains, intact or gets gradually thickened, and affords a permanent protection for the tumour.

Under these conditions it may be possible that the patient should survive and become fairly well developed, or may even reach adult life. I know of two cases of spina bifida in the lumbar region, of which one is already four years old and is in good health, despite that the tumour is as large as a pigeon's egg. In the other case there is paralysis of the lower extremities and of the bladder and rectum.

Treatment.-Though we have made great efforts to fulfil the two chief indications for the treatment of this deformity, the lessening of the tumour and the closing of the vertcbral canal, especially by surgical methods, we have not yet succeeded. The few cases of cure by art which are on record, form very rare exceptions. Puncture by exploring needles and subsequent injection of diluted tincture of iodine (Chassaignac and Braigmand), acupuncture, excision, écrasement (Gigon); finally, the systematic compression by means of collodion and other means, may now and then lead to a fortunate result, though oftener they hasten death. The most serviceable is the protection of the tumour from injury by a well-fitting concave plate of tin, strong leather, or gutta percha, for by this we assist the efforts of nature in the likeliest way. 


\section{Sensoro-neuroses and Hyperasthesia}

The following curtailed collection of the sensoro-neuroses of children is taken from the contributions of Valleix, Rilliet, Barthez, Henoch, Romberg, and Bohn, together with my own experience.

Cerebral neuralgia, megrims, hemicrania.-This occurs in children between the ninth and fifteenth years, more frequently in girls than boys, and is accompanied by pain which usually has its seat in the forehead and side of the head and continues for 'several hours, frequently associated with nausea, vomiting, photophobia, and giddiness. In a boy, ten years old and wellnourished, I saw the attacks return every four or six weeks, and always last for two days. Very frequently hereditary transmission is indicated as the cause, as in the instance referred to where the father was tormented with frequent hemicrania. In girls with indications of anæmia and chlorosis at the period - of puberty, the attacks of megrim or hemicrania sometimes persist until the appearance of menstruation. I have seen frontal neuralgia in children with ozæna, with cold in the head, and in some addicted to habits of masturbation. Bohn observed congenital left-sided hemicrania in a girl.

Occipital neuralgia.-I met with a distinct case of this kind in an extremely nervous girl, nine years of age. The neuralgia occurred for fourteen days, each day commencing an hour later, at first lasting from four to six hours, being especially severe during the night, and the beginning of the attack was accompanied by vomiting. The pain gradually diminished both in severity and frequency, large doses of quinine proving completely successful within the fourteen days. Enlargement of the spleen, as well as the other indications of intermittent disease, were absent.

Ciliar neuralgia is a rather common condition in scrofulous affections of the eye. Stinging pains in the eyelids, with more or less marked photophobia, are its symptoms ; and they may exist for only a few hours of the day, or have greater persistence. They yield to treatment directed against the scrofulous condition, the local application of blue ointment and extract of belladonna, and cold bathing or irrigation of the head. Counter- 
irritation by setons or blisters behind the ears or on the temples is also useful.

Neuralgia of the trigeminal nerve or supra-orbital neuralgia, with clear typical course, may be due to the influence of the malarial poison. In such cases there may be a preceding hot or cold stage, and the pain is increased by pressure on the orbital margin. Quinine and arsenic in the form of Fowler's solution, almost always afford relief.

Cervical neuralgia occurs in many cases of typhoid in infancy. I had under treatment a girl, ten years of age, in whom, from the second week till the convalescence from the disease, there existed a severe cervical neuralgia, the slightest movement of the neck producing great agony. This may often be observed in the beginning of tubercular meningitis.

Brachial neuralgia has been noticed by Valleix in a boy thirteen years old, as a result of burning of the last phalanx of the thumb. The pain, occurring spasmodically, originated in the thumb, and traversed the course of the median nerve, and yielded completely in six weeks to the use of carbonate of iron. A brachial neuralgia was completely cured by Henoch by iodide of potassium.

Neuralgia of the intercostal nerves occurs as a result of different pathological processes. Most frequently it accompanies dorso-pectoral herpes zoster, though it may precede the appearance of the eruption by two or three days. In the case of a delicate girl, aged eleven years, I found the neuralgia remain a fortnight after the disappearance of the vesicles, and its occurrence was most severe during the night. Intercostal neuralgia is not unfrequently due to direct irritation of the nerve-tissue by caries of the vertebræ. Such an affection I have known to last for eight years in the case of a boy aged fourteen years, who suffered from frequently recurring pain in the course of the ninth and tenth pairs of intercostal nerves. Chlorosis, also the disturbance of puberty and typhoid fever, may produce this affection.

Neuralgia in the lower extremities is met with at the onset throughout the course of coxitis, and in caries of the lumbar vertebra, when the pain is generally referred to the knee, the heel, or the ankle-joint.

Ischiatic neuralgia has been met with, by Bohn, in a boy of 
fifteen, and in that case it occurred every evening between five and seven o'clock and lasted throughout the greater part of the night. During the day all the movements of the limb could be made completely and free from pain, and the tracks of the affected nerves were not sensitive. There were some doubtful indications of feverishness.

Under the varieties of visceral neuralgia, on account of its frequency and severity, these rank first:-Enteralgia, neuralgia mesenterica or colic Sucklings, especially in the first months of life, are very frequently attacked by it. The paroxysms of pain are evidenced by violent piercing cries, retraction of the lower limbs on the abdomen, bending backwards of the head, convulsive closing of the hands, tense abdominal walls, congestion of the face, beads of sweat on the forehead, and frequently by passage of gas from stomach and rectum. Catarrh, dyspepsia, flatulence, gastro-intestinal catarrh and constipation, are the lesions to which it may be due. Worms, especially the Ascaris lumbricoides, may excite more or less severe attacks of it in older children. The seat of the pain is usually the umbilical region.

Cardialgia is not often seen in children : it occurs independently of intestinal lesion in girls between nine and fourteen years of age as a concomitant symptom of the disturbance of the system during the access of puberty, and then it is very obstinate. I have seen it continue for two years with only slight intermissions, and it is often accompanied by or may alternate with urticaria. It may be produced by the presence of worms, especially the tape-worm. In the case of an anæmic boy, nine years old, I saw recurrent cardialgia with symptoms of cerebral disturbance, that gave rise to anxiety; these lasted eighteen months, but yielded completely to a course of chalybeates.

Vesical neuralgia is for the most part due to the presence of stone in the bladder, and varies much in degrees of severity. I have seen it in the course of vesical catarrh, with croupous inflammation of the bladder during typhoid fever in the case of a six-year old boy, in tubercular disease of the bladder, and in some children affected with caries of the vertebral column. The irritation of worms and masturbation are said by Pitha to produce this neuralgia. 


\section{Neuroses of Motor Power}

\section{(a) Eclampsia, Convulsions, Fits}

Under the term Eclampsia we include those clonic and tonic spasms which affect sometimes only a few, sometimes a large number of the voluntary muscles, and in which consciousness and mental activity are more or less interfered with. The eclamptic attack, considered by itself, can hardly be distinguished from epilepsy; and in this sense we may perhaps regard eclampsia as acute epilepsy; or if epilepsy become chronic it may be regarded as recurrent eclampsia, without any logical contradjction. In the one case we have a condition either passing quickly off in recovery, or having a fatal termination; in the other a chronic condition, when from time to time some irritating cause produces the group of symptoms; and it may happen in children that really only the after-course, the return or the absence of convulsions, can afford an indication of the true nature of the case.

Causes, symptoms, and course.-All convulsions are symptomatic, and may be classed as direct or idiopathic, and indirect or symptomatic (the so-called reflex convulsion), according to the seat of the irritation; in the former case it will be in the nervous centres, in the latter in the peripheral expansions of the nerves.

The causes which produce direct convulsions are manifold, and I can only briefly notice them under the following anatomical groups :

1. Convulsions from arterial hyperæmia of the brain and its membrancs are found to occur in various acute diseases where, by increased temperature, such hyperæmia is produced. To these belong the convulsions resulting from insolation, those occurring at the beginning of meningitis, and sometimes the convulsions of teething children, though the latter may have their origin in a reflex process.

2. Convulsions from passive hyperæmia are such as may be observed in the course of hooping-cough, more rarely in lobular pneumonia, in exudative pleurisy, croupous laryngitis, organic disease of the heart, and severe constipation of the bowels. 
3. Convulsions from anæmia are quite as often met with as those due to hyperæmia, and are especially frequent in acute general anæmia of the brain after great loss of blood or of the humours of the body. They may be also induced by partial anæmia of the brain, the result of tumours, or the expression of a probable vaso-motor paralysis.

4. Convulsions from qualitative alteration of the blood form a rather numerous group, and include most prominently those occurring in such zymotic affections as scarlet fever, mcasles, smallpox, typhoid and intermittent fevers, which not unfrequently set in with severe convulsions or are accompanied by them in their course. The younger the child and the more intense the attack, the more readily are convulsions induced. In these cases it must not be forgotten that cerebral hyperæmia has some part in the production of the convulsions, as well as the alteration of the blood which we call specific for want of knowledge enough to enable us more satisfactorily to define it. By such combination are produced the convulsions of uræmia, cholæmia, diphtheria, and intoxication with certain drugs and poisons (as carbonic acid poisoning).

5. Convulsions from disease of the brain and its membranes are such as occur in meningitis, hydrocephalus, œdema of the brain, encephalitis, tumours of the brain, intra-cranial hæmorrhage, and they only too frequently form the closing scene of such diseases.

6. Convulsions from diseased alteration of the brain-case are seen chiefly in rickets with and without craniotabes, chronic hydrocephalus, and microcephalism.

\section{Indirect, symptomatic, or reflex convulsions}

There are many sources of irritation, inconsiderable in themselves, which lead to convulsions in children by reflex action, as digestive disturbances, especially in sucklings, overloading of the stomach, dyspepsia, intestinal catarrh, obstinate constipation, and probably the presence of worms. Convulsions in sucklings consequent on mental suffering on the part of the mother belong also to this category. Other reflex irritations may be instanced in scalding, as in over-heated baths, burning, 
stings, abscesses, \&c. I have seen convulsions occur in the course of an abscess in the neck, in the case of a strong, wellfed child; and these completely ceased after the abscess had been opened. Otitis, foreign bodies, as insects, in the external auditory canal, inflammatory diseases of certain organs, as pleurisy, peritonitis and pneumonia, may be the source of reflex irritation. The reflex convulsions which attend the teething process are well known; but it behoves us to be cautious, and not to set down any eclamptic attack which may occur in a teething child as taking its origin through the trigeminal nerve.

Finally, we must lay stress upon hereditairy transmission as one of the causes of convulsions, for it has been well pronounced in many instances. - This consists in a diminished capacity for resistance in the whole nervous system, and is displayed very frequently, though not always, as a tangible element in the constitutional character of such individuals and families.

The convulsions occur, as one or other of the aforementioned causes may be the excitant, either suddenly without the existence of any previous disorder, or they may be preceded by certain premonitory symptoms, such as indications of despondency or impatience on the part of the child, whimpering, and its sleep being broken by frequent startings and jumpings. Rapid changes in the child's complexion are often noticed as early indications of an outbreak, the face being sometimes pale and sometimes red; occasionally there is a well-marked blue line round the lips and slight muscular tremors in the face.

The number of the eclamptic attacks varies very much, though they are rarely confined to a single paroxysm, more frequently following one another in rapid succession, alternately with short intervals free from the spasms. A single attack may last from a few minutes to several hours, and the longer its duration the more suspicious will be its significance.

Accordingly as the centre of irritation is central or peripheral, small and cireumscribed or diffuse, and giving rise to various lesions, so will the convulsions be partial or general, in one case attacking different groups of muscles, and in another always returning to the same.

Treatment.-An accurate investigation of the exciting causes of the convulsions with especial reference to the condition of 
health of the child previous to the attack gives the most important indications for treatment, though this is not always easy, and may often require longer observation than may be possible, that we may estimate rightly the importance and true indications of the convulsions.

Considering this, it is evident that causal treatment must often give place to symptomatic. An accurate examination of the child, either during the attack or at its conclusion, will, under any circumstances, be urgently demanded, and may be of great value, especially in reflex convulsions. Removal of all constricting articles of clothing or bedclothes and the freest possible admission of fresh air, are the first duties of the attendant. When the administration of remedies by the mouth becomes from any reason impracticable, derivation from the skin and intestinal canal must be employed, for which purpose clysters of cold water, or containing vinegar or assafœetida in cases of flatulence, together with rubbing of the skin with vinegar and water, the application of mustard poultices to the trunk and extremities and the use of cold wet packing; a warm bath also frequently allays general convulsions, especially in teething children. For the rest, the generally employed remedies are the preparations of zinc, especially the oxide, either alone or in combination with calomel (four grains of the white oxide, two grains of calomel, with a drachm of white sugar, to be divided into eight powders, one of which is to be given every hour). Arsenic, also the nitrate of silver, or Dover's powder, may be tried. Bromide of potash, given in doses of from one to two grains every three or four hours, has not proved of much use in my experience, and I have gone back to the use of the zinc. Digital compression of the carotids, highly spoken of by Bland and Trousseau, can only at most find a recommendation in convulsions from active hyperæmia, and its use is of doubtful propriety. From my own experience I cannot speak favorably of this method of treatment.

In the convulsions of toxæmia, especially in the course of intermittent fevers, quinine in large doses is to be employed, and it has sometimes seemed to be of great service in uræmic convulsions. If there are any reasons for suspecting the presence of worms, or if they have previously been passed, anthelmintics ought to be administered. For convulsions which have their 
origin in deeply-seated brain lesions, as a rule, we can do little or nothing.

\section{(b) Trismus and Tetanus-Lockjaw}

Tetanus is a motor-neurosis which shows itself in tonic con.. actions, sometimes of isolated muscles, sometimes of the whole voluntary muscular system. When it occurs only in the masseter muscle it is known as trismus. Trismus and tetanus usually occur together in children, the former being always accompanied by trismus, though the latter may be observed without tetanus. The disease often does not arrive at full development, and this is most commonly the case in new-born children. I have seen it in fifty-two cases in twelve years, and of the number forty were newly born and twelve were older children with pure tetanus.

Anatomy. $-\mathrm{U}_{\mathrm{p}}$ to the present time post-mortem examinations have not yielded any results by which has been established any lesion that can be regarded as the essential of the disease. Congestion of the brain and spinal marrow have often been observed, also serous and sanguincous effusions in the spinal arachnoid sac, the latter by myself, and are more probably results than causes of the disease, though in the case of the hæmorrhage I might have regarded it as the final cause of the tetanus. According to Hirschberg interstitial encephalitis forms an anatomical basis for trismus neonatorum. The development of connective tissue in the spinal marrow observed by Rokitansky and Demme is not common to all cases. More frequently are found signs of violence or an incompletely cicatrised umbilicus.

Symptoms and course.-In new-born children (trismus neonatorum) the disease indicates itself as follows:-Between the third and eighth day after birth, after premonitory symptoms such as restlessness, whimpering, broken sleep, yawning, and hasty snatchings at the mother's breast, which is, nevertheless, soon loosened, there sometimes appears an inability to open the mouth. The features are peculiarly altered, the lips thereby stretched apart, the eyes shut close, and the alar cartilages move tumultuously. Gradually the spasms extend with intermissions down the neck and spinal muscles, finally even to the extremities, so 
that the child lies still like a statue, and may be lifted like one. From the inability to suck or take nourishment the patients visibly emaciate, the intervals between the paroxysms become more seldom and shorter, the pulse rises to 140 or 160 , a cold clammy sweat breaks out over the skin, and death results in a few days, at the outside eight, after the onset of the disease.

Tetanus in more advanced children occurs either originally along with lockjaw, or it may be ushered in by certain premonitory symptoms, as irritability, dragging pains in the neck, transient shiverings, pains in the throat, and impeded movements and speed. Trismus and general tetanus rapidly follow upon these indications. The muscles of the neck, trunk, and extremities are stiffly contracted, any action or passive movement of them being impossible, while sensibility is increased or unaltered. The temperature is generally increased during the further course of the disease, though at approaching death it sinks below the normal line; the pulse is frequent, small, and later on it is irregular, as is also the breathing. Of the various forms of tetanus, I have most frequently seen orthotonos, in which the body is stiffly stretched out ; more rarely opisthotonos, when the body is arched upwards from behind; and most rarely emprosthotonos, when it is arched backwards. At first paroxysms alternate with remissions, but gradually the former become permanent, or at most become only temporarily relaxed into slight convulsive tremors. The mental activity generally remains undisturbed until shortly before death, though I have seen clouding of the intellect, or even complete loss of consciousness, for fifteen or twenty hours before death.

The result is usually fatal in a few days, or in from two to three weeks, though exceptionally the disease ends in recovery, and such an ending may be hoped for when the remissions become longer, and are free from muscular tremor, and when sleep and the possibility of movement are again established. Of the 52 cases which have come under my own observation 45 have been fatal.

Causes.-The immediate cause is still doubtful. According to the exciting causes we may distinguish as varieties of tetanus in children the traumatic, rheumatic, and toxic. Irritation of the sensory fibres, of the peripheral nerves by various noxious external influences, producing reflex muscular spasms, is, 
perhaps, the most frequent process in the development of tetanus. Tetanus neonatorum is sometimes traumatic, sometimes probably rheumatic. Vogel's assertion that it always depends upon the process of closure of the umbilicus has been refuted long ago by observation. In some cases the stump of the umbilical cord may doubtless be the point of origin of the disease, and then it is of course traumatic; and to this class also belong such cases as have their origin in ritual circumcision and burns (Bohn); to the other belong those due to the influence of great cold on the tender skin of the new-born child, as by the splashing of a still-born child with cold water, a bath at too low a temperature, and, perhaps, also the influence of cold draughts. I have recently met with a case in a child fourteen days old, when trismus and tetanus set in after the use of a bath ton hot, and had a fatal issue in three days. The observations of Keber, of Elbing ('Monatschrift für Geburtskunde,' 1868), also show this, for he narrates that a midwife, from a want of proper appreciation of the temperature of the water in which she washed the children, used it too hot, and in the course of two years among 380 births, lost 99 children of tetanus. Advancing experience also shows that bad, poisonous, and smoky air in the room occupied by the children favours the development of tetanus.

Trismus and tetanus occur during childhood from wounds of different kinds, sometimes of the most trifling importance. Stings, penetrating wounds of the hands and feet by pieces of glass or splinters of wood, or by treading on a sharp stone, fractures of bones with fissuring, violent distortions of the vertebral column, may be met with as exciting causes of traumatic tetanus. Toxic tetanus in children comes more rarely under our notice. I have met with one such case in a ten-yearold boy subject to epilepsy, after the administration of a grain of atropine, and in whom, after lasting three days, the tetanus again disappeared. Rheumatic tetanus may be the result of sleeping on the cold, damp earth, bathing while the body is heated, \&c. In tropical countries tetanus occurs endemically and epidemically, while with us it is seen only in its sporadic occurrence.

Trealment.-The majority of observers agree that we have no specific against this disease, fearful in all its forms, and that 
our power is limited to a treatment which can be only prophylactic or palliative. Caution is necessary in the first dietetic treatment and nursing of new-born children, especially with reference to the atmosphere in which they are reared, and the baths in which they are cleansed, and in the treatment of the umbilical cord, and in the ritual act of circumcision; suitable surgical treatment of wounds will doubtless diminish the number of cases of tetanus. When the disease has become established, such remedies as opium, morphia, curara and Calabar bean, administered either internally or by subcutaneous injection, are most to be advised, though even they will generally lead to disappointment. Cold wet packing seemed to me in several instances to diminish the intensity and frequency of the attacks, though in other cases it seemed to have just the contrary effect, and warm baths seemed of greater service. 'The worst results are furnished by the tetanus neonatorum.

\section{(c) Spasmus nutans ; Nodding Palsy; Salaam convulsion of infancy}

This affection shows itself either as only unilateral clonic spasm, in exceptionally rare cases affecting only the region supplied by the spinal accessory nerve, or bilaterally with symmetrical implication of other nerves. Spasmus nutans deserves the name of a spinal neurosis only in occasional cases, as Ebert has insisted in his observations relating to this condition When unilateral the disease is characterised by contractions of the sterno-cleido-mastoid and trapezius muscles of one side, occurring in fits and starts, sometimes with considerable frequency, by which the head is drawn strongly backwards and forwards, and the shoulder is raised. I have seen two cases of this kind, one occurring in a boy nine years old, previously healthy and well-nourished, in whom it was alleged the lesion appeared after exposure of his neck to a strong draught. The spasm on the left side was very severe, had generally only short intermissions, and after lasting three weeks, yielded to cold water applications. The second case was that of a girl at the period of sexual development, who was of a particularly excitable disposition. In her the right side was affected, the 
contraction disappeared completely during sleep, was rendered more severe by any mental excitement, and disappeared entirely in six weeks after the exhibition of iron and zinc.

Bilateral, or the true salaam convulsion, occurs in the more severe form as regular and frequently repeated nodding, and then may have a very grave significance as the accompaniment of serious lesion of the central nervous system; though its importance may, on the other hand, sometimes be very slight. It is often seen in children between seven and twenty months old, and is not unfrequently associated with spasmodic affections of the muscles of the eyes (nystagmus, nictitatio), and may, in a certain class of cases, be associated closely with difficult teething and rickets. The presence of worms, and indigestion have also been pointed out as causes. Consciousness is never disturbed in the slight and transient cases, but it is otherwise when the convulsion is merely the symptom of nervous lesion. In the latter case it seldom occurs alone, but is accompanied by other symptoms indicative of nerve lesion, as clouding or loss of consciousness, convulsions affecting other muscle groups, widely dilated pupils, \&c. I saw a case of this kind in a ten-year old girl, where a tumour on the under surface of the cerebellum and pons produced the salaam convulsion to a very severe extent.

Prognosis.-This varies as the cause is transient or deepseated and irremovable. If it depends on rickets, the process of teething, or the presence of worms, the prognosis will be favorable.

In slight cases independent of brain lesion the treatment may consist of oxide of zinc, iron, cod oil, and bromide of potash; but if thcre be central mischief all treatment will be unavailing.

(d) Chorea minor; Chorea Sti. Viti; Ballismus; St. Vitus' Dance; Muskelunruhe

It is not yet possible to give a definition of this disease that will include the essentials of all cases. We include under chorea minor any pathological condition of the central nervous system, especially of the spinal cord, which has the result of 
disturbing more or less the influence of the will over voluntary muscles, and where involuntary movements occur which usually subside during sleep, and by which consciousness is undisturbed. Chorea minor is frequent during childhood, and I have seen 275 cases in ten years.

Anatomy.-As this disease almost invariably ends in recovery, the results of pathological investigations concerning its nature, and the alterations to which it is due, are still sparse and somewhat contradictory.

Of four fatal cases which came under my own observation, in one an increase of the connective tissue of the spinal cord was found; in a second an extravasation of blood in the spinal canal, and in the third a serous effusion; while in the fourth the result of the section was entirely negative. Serous and sanguineous effusion in the vertebral canal have been found by West and Prichard ; softening of the spinal substance, by Gendron ; membranous formation in the cerebellum, by Soemmering, cerebral tubercle, by Georget; and elongation of the odontoid process and consequent pressure on the spinal cord, by Froriep. Cerebral embolism has been very recently observed by several authors, especially in cases where the chorea has been complicated with disease of the heart.

Symptoms and course.-The disease is often ushered in by premonitory symptoms, as lassitude, great irritability, complaining disposition, palpitation of the heart, awkward behaviour, uncertainty of movement and grimaces, \&c. More rarely it occurs suddenly, as muscular restlessness. At the onset there are noticed slight tremulous movements of the muscles of the face, especially of the orbicular muscle of the mouth, of the shoulders and hands, and very often rapid protrusion and withdrawal of the tongue. As the disease advances, these involuntary muscular movements increase in extent and frequency; the hands are no longer able to seize and retain hold of objects-in piano playing the fingers spring over the keys and are unable to strike a chord; the patient can no longer eat unassisted, but carries the spoon or fork to the nose, to the eye, or altogether below the face. The arms are twisted about hither and thither, and this extravagance is accompanied by absurd grimaces. The gait is unsteady and tottering, one foot striking against the other. or the feet are cast about 
irregularly; and if the patient is asked to extend his hand, he makes a movement with his feet and draws himself round in a half circle. Later on in the course of the disease the patient is no longer able to stand or walk, but must lie, and even in that position will be tormented with the muscular restlessness; he strikes out with his hands and feet, and so rubs and scratches the skin of his face and other parts of his body as to produce sores and decp excoriations; stuffs his fingers into his nose so that it bleeds, and undergoes various other contortions. Sometimes the back is arched, simulating opisthotonos.

In the majority of the cases the affection extends to the whole of the voluntary muscles, though it is sometimes unilateral, and then the left half of the body is more frequently attacked. Occasionally it is limited to the muscles of one arm, and exceptionally the muscles of respiration are the seat of the contractions. The implication of internal muscles is shown by difficulty in extending the tongue, which, indeed, can be done only momentarily and by quick, interrupted movements, also by difficult and stuttering articulation, and irregular contractions of the diaphragm. Irregular action of the heart has also been noticed. The involuntary motions cease during sleep in mild cases, though in the more severe the patient gets no sleep, or his sleep is very unquiet. The sensibility of the skin is seldom altered though it is sometimes diminished in unilateral chorea, consciousness is never clouded though the intellect is often disturbed, the child becomes irritable, complains groundlessly, is easily frightened, and tears and laughter causelessly alternate.

In fatal cases the muscular excitement abates markedly or ceases entirely during the last few days of life, and tetanic contractions of the muscles of the trunk and extremities are substituted, together with subsultus tendinum, slight convulsions, and finally coma.

If the chorea coexist with disease of the heart death may occur from œdema of the brain, of the lungs, from hydrothorax, effusion into the pericardium, \&c. Cardiac murmurs in the course of chorea are often only the result of anæmia. The disease often runs a protracted course, its shortest duration according to my experience being fourteen days and its longest two years and fourteen weeks; and the average time from four to nine weeks, though exceptional cases have been met with where 
it has lasted throughout life. I know two men between fifty and sixty years old who have suffered from chorea since they were about eight years old.

Relapses are frequent, and the disease returns in many cases at intervals of months or years. Some authors have noticed a mental weakness remain after chorea, and in three cases I have seen it followed by epilepsy.

As a rule, the course of chorea is free from fever, though in rapidly fatal cases a high temperature curve may be met with. Painful sensations along the course of the spine are frequently, though not constantly observed. Intercurrent acute diseases, as scarlet fever, measles, smallpox, typhus, and diphtheria, may alter the course of the chorea or cause its disappearance.

Causes.-The immediate cause of chorea is a spinal irritation, excited and kept up by various anatomical conditions, as anæmia, hyperæmia, serous exudation, sanguineous extravasation, new formations, and organic alterations in the course of the spinal marrow and its membranes, such as osseous growths. This spinal irritation may be of traumatic origin, be induced by rheumatism, or be the result of altered growth or development. Whatever the connection between chorea and acute rheumatism may be, it cannot be denied that the two diseases often occur shortly after one another, though this is not always the case, as Roger believes, and therefore the chorea cannot always be considered as only a partial phenomenon of rheumatism. The connection seems to be in the preference on the part of rheumatism for attacking serous membranes, while in chorea the affected part is the spinal meninges. We are not yet justified in adopting embolism as an explanation of all cases of chorea. For the most part chorea is the expression of certain abnormal conditions of growth and development to which belong the changes of dentition, the development of sex, rapid growth, delicate constitution, and general anæmia.

Intestinal worms have been instanced as the exciting cause of reflex chorea, but I have known of no such case. Amongst the essential causes must be mentioned certain predisposing and exciting factors, the so-called procatarctic causes. To the first class belongs the period of life, between the sixth and fourteenth years (the youngest of my cases was three, the oldest fourteen years old); the influence of sex, for of 275 cases I found 
214 were girls and 61 were boys; and also hereditary disposi. tion and certain climatic relations, for the largest number of cases occurred in the months of January and February.

Mental influences act as exciting causes, for if after sudden fright, fear, sorrow, intense joy, \&c., in a child, chorea should appear, we may consider that a certain condition of irritability of the spinal cord has existed for a longer or a shorter time, and that the mental influence has only added the last impulse necessary for the outbreak. To these may be added also certain mechanical influences, as a fall, injury, or blow, which, partly alone, partly in combination with the mental excitement, may favour the outbreak of the chorea.

Epidemic outbreaks of chorea have been observed, and their explanation sought in imitation (Bricheteau).

I noticed an epidemic of chorea in the winter of 1870 (nineteen cases in the course of five weeks), and regarded the unusual condition of the weather as the origin of the exceptional frequency of the disease, as it certainly may be regarded as an exciting cause. The majority of the children had previously suffered from chorea and imitation I excluded as not possible.

Prognosis.-This is generally favorable, though we must remember the possibility of the recurrence of the disease, and if this should be frequent the presence of some incurable lesion of the nervous centre may be suspected. It has been generally admitted that recovery is more difficult and tedious in boys than in girls.

Treatment.-It is admitted that the disease will get well of itself without treatment after a certain duration, but experience has also shown that by certain remedies it can be made less severe and more bearable and may be much shortened.

Sometimes it is possible to conduct the treatment on an xtiological basis; and as chorea may be considered in the majority of cases as a disturbance of nutrition, growth, or development, a view that is supported by the general presence of anæmia, the fundamental treatment must, be chalybeate, either simple or in combination with quinine or oxide of zinc. Along with these arsenic, especially in the form of Fowler's solution, may be mentioned as being of service, given in doses of two or three drops twice or thrice daily and cautiously increased. When there is great restlessness the combination of opium and 
Fowler's solution is often surprisingly beneficial. Bromide of potash though commended by many writers, has not in my experience been so useful as the above remedies. If the chorea has a direct relation with a rheumatic attack, either present or past, then the remedies indicated are those adapted for the latter disease, as quinine, digitalis, and opium. Cold water applications, in the form of packing, rubbing, or douche, have proved of service in some cases of a nature not easily defined, while to others it seems to have done harm ; and in such; on the contrary, warm baths, either with or without sulphur ingredients, have done good. If it can be ascertained that intestinal worms exist, anthelmintics should be used.

The employment of electricity, especially the constant current, has been recently said to be valuable as a remedy, but I have had no personal experience of it. The curative gymnastics employed by English and French practitioners are to be recommended.

Children affected with chorea, especially when there is great muscular restlessness, sleep most safely on a mattress laid on the floor of the room. If they remain in bed they must be protected on all sides by pillows. Mental excitement of any kind is strenuously to be avoided, and the patients are not to be excited by mixing in a numerous company, but must be kept quiet, and to a great extent isolated.

\section{(e) Chorea Magna-German Chorea (Grosser Veitstanz)}

Chorea magna is a rare affection, and essentially different from the chorea minor. It occurs in paroxysmal disturbances of the musculo-motor power, and also of the mental functions, so that consciousness seldom remains intact, but is to some extent or wholly destroyed. The motor lesions occur as numerous movements, apparently voluntarily performed, and which, though rash and hazardous, may be accomplished with astonishing accuracy. The children climb, spring, creep, sometimes dance rapidly round in circles, and in other cases are seized with epileptiform convulsions or cataleptic rigidity. The mental signs consist of indications of exultation, the face lights up, the eyes brighten, the patient begins to sing briskly, to declaim or preach, or to hold extraordinary conversations with some one 
not present; others, again, show indications of terror, imitate the cries of animals, \&c. Outbursts of religious frenzy and terror of the unseen exist as not infrequent characteristics of this mania.

The paroxysms generally occur suddenly, rarely are preceded by indications of mental irregularity, last only from a few minutes to a quarter or half an hour, or even a few hours, and then disappear, and the patient wakes as if from a dream, with astonishment, or he falls into a deep sleep after a longer or shorter interval, and afterwards has no recollection, or only a very dim one, of the attack. The temperature and pulse are usually increased during the paroxysm and are lowered again towards its conclusion, and the patient shows signs of nervous exhaustion in the intervals between the attacks.

The essential condition of this affection, as also those of the related diseases, somnambulism, animal magnetism, \&c., is still unknown. If we consider that chorea magna is always a transient curable affection, and is certainly only seldom, as several observers remark, developed into distinct epilepsy, that these exceptional cases occur between the tenth and sixteenth years of life, also during sexual development, that the majority of the children attacked belong to the female sex, and that the disease generally disappears after the occurrence of the catamenia; we are induced to regard it as a motor-psychic neurosis, arising from the sympathetic nervous system, and that it shows itself in one or other of the fashions described above, according as the motor or psychic brain-centres form the points of origin of the attack.

The changes of puberty, the female sex, perverted education, too exciting reading, the bad example of a nervous mother, precocious love dalliances, and confinement in monastic seclusion, are to be regarded as predisposing and procatarctic causes.

One case, of my own observation (communicated in the 'Jahrbuch für Kinderheilkunde,' $2 \mathrm{Heft}, 1864$ ), occurred in a delicate orphan girl thirteen years old, who had been brought up by her aunt, a nun, in the cloister. The paroxysms lasted from a quarter to half an hour, occurred indifferently by day or night, and consisted, during their first stage, of the exhibition of religious ecstasy, with risions, singing sacred songs, declamations, conversation with God, the Virgin, and the angels, 
and they were followed by cataleptic torpidity. After six weeks of treatment by iron and zinc and permanent removal from the cloister the girl recovered, though the disease had existed six months. Menstruation occurred soon after.

In the investigation of this disease great discrimination is required to avoid deception by simulation.

Treatment. - If the affection is associated with amenorrhœa or dysmenorrhœa, then iron, either alone or in combination with zinc, is the appropriate and most useful remedy; but if no cause can be detected, then zinc, or, perhaps, the bromide of potash, will be of most service. It is very necessary that the child should be removed from her previous associations, and that all other procatarctic causes be avoided, and that her physical and mental regimen should be carefully regulated.

\section{(f) Chorea Electrica}

Chorea electrica is any motor neurosis by which, in single muscles or in groups of muscles, violent and fitful contractions occur, instantaneous in their duration and rapid in their sequence, resembling those produced by the galvanic current. Consciousness is in no way affected, and during sleep the contractions cease. These movements, which by preference seem to attack the upper and lower extremities, may also be exerted by certain voluntary movements. Thus, I have observed as a rare phenomenon this peculiarity in a pair of twins, girls of seven years old, who both had it. Thus, as often as the children sat down on a stool there occurred, instantaneously, most violent electric movements of the upper limbs, and ceased only when the girls stood up.

The disease affects chiefly delicate anæmic girls with excitable nervous systems, in the period between the second dentition and the appearance of menstruation.

The exciting cause is, in all probability, a condition of irritation of the nervous centres, especially the spinal cord, and the movements are produced sometimes directly, sometimes by a reflex process. The disease generally lasts some weeks or months, and results in recovery.

Diagnosis can generally be made by the peculiar movements 
which occur always with a certain regularity and symmetry on both sides of the borly. It is scarcely possible to mistake it for chorea minor or magna, on the most superficial examination of the symptoms.

The treatment consists in the administration of the liquor arsenicalis, oxide of zinc, or the bromide of potash, in increasing doses, and when anæmia exists iron is to be given simultancously. A suitable hygienic and dietetic treatment of the child is, of course, necessary.

\section{(g) Epilepsy-Falling Sickness}

The falling sickness is a common disease of childhood, and very often the epilepsy of adult life may be referred as far back as the later years of childhood. In the children's hospital at Prague, according to Löschner, 242 cases occurred in ten years out of 7000 sick children.

Anatomy.-Post-mortem examinations have revealed so many various essential alterations that the conclusion has been arrived at that there is no specific lesion by which the disease can be accounted for; thus there have been found microcephalism, hydrocephalus, and arrest of development of the brain in the new-born; tumours, abscesses, hypertrophy and atrophy, softening and sclerosis, embolism of the cerebral vesscls, \&c.; and similar lesions of the spinal cord, as causes of epilepsy in adults, while sometimes the results of the section have been wholly negative. Among the rare causes I have found a hr.. matoma internum in a child two years old, of which the origin probably could be traced back to an intra-meningeal apoplexy, produced during birth, as the epilepsy had occurred since that time at longer or shorter intervals.

Symptoms and course.-The epilepsy of children often differs from that of adults in that the paroxysms take only a slight form, or are even barely indicated, and only in the course of years increase to any degree of severity. At the outset of epilepsy we often see a mere slight vertigo, or a distortion and sudden blanching of the face lasting hardly a second, or that in walking across the room the child gets into a swoonlike condition, and quickly sceks for some support. As a premonitory symptom of subsequent epilepsy, I have seen purposeless con- 
vulsive contraction of a finger, which again disappeared after lasting for a few minutes.

Whilst then these slighter foreshadowings of the disease almost always appear without premonitory symptoms, we find the more severe and perfectly formed symptoms frequently preceded in children by prodromata, such as despondency, sad: ness, monosyllabic talk (Einselbigkeit), disposition to drowsiness or increased irritability, pain in the head and indications of the aura epileptica, especially in older children near puberty. The completely formed paroxysm shows itself by sudden loss of consciousness, happening often with a barking, inarticulate cry, and by the falling down of the child in alternating tonic and clonic muscular convulsions of very various kinds and characters. Froth and bloody mucous are often seen at the mouth, and both fæces and urine may be voided involuntarily; and after the convulsion has lasted a longer or shorter time, the child awakes with a few deep inspirations as if from a dream, looks vacantly aruund, and has no recollection of what has happened; or it may fall directly into a sleep.

The severity and frequency of the attacks not only differ in different cases, but also vary in one and the same patient in a remarkable manner without evident reason. The fits occur by preference during the night time, and therefore often escape observation. I have known in several cases as many as forty fits in the twenty-four hours ; in others they occur only at intervals of three or six months, or they may return with intermediate pauses of years.

Wounds, especially of the head, biting of the tongue, and contusions elsewhere on the body, are often produced during the fits. The course of the disease is almost always very chronic, very often life-long. During its existence the mental activity of the child may be well developed, even to the indicacation of talent; while in others it is much interfered with, even to the establishment of idiocy or mania.

Causes.-As I have already said, the exciting cause is not one and the same for all cases, and for many it remains entirely unknown. It is more than probable that in the majority of cases of epilepsy there is some anatomical lesion for its basis, and that the so-called idiopathic epilepsy, at least in infancy, must always be very uncommon. Enlargement of the blood- 
vessels of the medulla elongata (Schröder van der Kolk), acute, especially spasmodic, anæmia of the brain (Kussmaul and 'Tenner), are said to induce the attack. We assume that as a result of rapidly induced cerebral anæmia, which may occur either directly from a brain lesion, or by reflex method, consciousness is lost on the one part, while on the other, the convulsion centre, seated in the pons, is irritated and the epileptic seizure is the result. Why there should occur in one individual slight and infrequent attacks, and why in another they should be frequent and severe, we do not know. Reflex epilepsy, such as is produced by cicatrices, especially in the scalp, by retained foreign bodies, by diseases of the genitals, by intestinal worms, \&c., is certainly uncommon in childhood; at least according to my own experience this rarity exists in the case of worms, which are often enough blamed for the disease in children; for I have not seen a single case produced by that cause. There can be no doubt that hereditary influence is powerful in this affection, and that the disease passes from parents to children, sometimes passing over a generation and becoming atavic.

Treatment.-Our endeavour first should be to discover and remove, if possible, the exciting cause, though this can be done only seldom. If helminthiasis or delayed menstruation seem to occupy the position of cause, then anthelmintics or preparations of iron are to be employed. If any concomitant symptoms of scrofula or tuberculosis indicate cerebral tumour, then cod oil, iron, and iodine, are to be administered. In the usual absence of indications of the cause we are confined to the use of some of the numberless empiric remedies for the disease, which almost always have the common fate of remaining in favour for a short time and being then confined to the curiosity cupboard. To these belong the salts of zinc, nitrate of silver, ammonio-sulphate of copper, and atropine. The last is not a safe remedy for children, and has given in my experience no results, despite a various and long-continued trial. It must be begun in small doses, but I saw $\frac{1}{7}$ th of a grain employed in a case of tetanus occurring in a boy ten years old. The bromide of potash has been highly spoken of in recent times, and has come to be the usual remedy employed for epilepsy. Weakening and diminution of the paroxysms I observed in several 
cases, especially when after large doses a degree of saturation seemed to occur; but I have not as yet to thank bromide of potash for a case of permanent recovery. Of curara, recommended by Benedict, I have not yet had any experience, though I very much wish that I may be deceived in my expectations of it. The experience recorded by other practitioners that any new remedy seems for a time to have a preventive influence over the disease, I can confirm, and herein lies the encouragement frequently to change the remedies in the treatment of such cases.

\section{(h) Akinesia-Motor-Paralysis-Palsy}

Paralysis occurring in children may generally be traced, as in adults, from two great sources of origin-either lesion of the central nervous system, the brain and spinal marrow, or disturbance of and hindrance to the transmission of the currents in the nerves from their central origin to their peripheral distribution. $\Lambda$ rigid classification of these affections, from their symptoms, is not as yet possible, so that I shall better review them by grouping them as they have occurred in clinical experience.

\section{Essential Paralysis in Children-Spinal Palsy}

This belongs almost exclusively to children, and is the most frequent form of paralysis in them; it consists in partial or complete loss of motor power in one or all of the muscles of the upper or lower extremities, generally one limb, seldom both. The sensibility and contractility under the electric stimulus is unaltered by it, or they may be weakened or altogether destroyed in certain muscles or muscle-groups. Sometimes the paralysis occurs without premonitory symptoms, and may be developed in a night's time without any other lesion, though more generally there may be noticed for a few days or a week before slight feverish symptoms-a certain uneasiness and restlessness, especially at night; sleeplessness or stupor may also be noticed, and these, after the occurrence of the paralysis, generally disappear. If cerebral symptoms appear, they generally pass off quickly. The destruction of motor power is either incomplete. 
(paresis) or complete (paralysis). If it occurs in an arm the limb hangs helplessly down, or is used by the child with the assistance of the sound arm; if the muscles of the upper arm alone are affected, as sometimes happens, the child is able to seize objects, but cannot lift them up. If a lower limb is affected it is noticed that when the child is asked to stand or when undressed it draws one limb up on the abdomen, but lets the other hang flaccid. These paralyses disappear only in very exceptional cases, but they may do so according to some authors (Rilliet, Barthez, Kennedy, and West) so soon as within fiom two to eight days : as a rule they remain stationary, and lead to fatty degeneration of the muscles, and the contraction of these results in paralytic clubfoot, flat foot or talipes equinus, in more or less severe degrees ; otheruvise the condition of the child is generally satisfactory, its growth and education advancing, whilst sleep, the appetite and the mental activity go on normally developing.

The actual cause of this paralysis is to be found, doubtless, in a material alteration of the spinal cord, though this has been demonstrated in only a few cases (Heine, Vogt). According to my own view different causes may produce and maintain this paralysis, as new connective-tissue formations, effusions of blood, inflammatory processes in the course of the spinal marrow, and at least the obstinacy and incurable nature of the affection would indicate such origins. It occurs for the most part in children during the first three years of life, by my own experience more frequently in girls than in boys; but this is, perhaps, only accidental, for Vogt has found just the opposite. I think the process of dentition can scarcely be placed in etiological relation with it, though in most of the children $I$ found decided evidences of rickets.

\section{Diphtheritic Paralysis}

This may occur during the course of diphtheria, but more frequently it comes on during the first few days or weeks of convalescence. It generally is seen first as paralysis of the soft palate with difficulty of swallowing and indistinct nasal utterance. 'This may be followed by paralysis of the vocal cords, of accom- 
modation of the eyes resulting in diplopia, paralysis of the limbs, more frequently of the upper than the lower, and of the bladder and rectum.. Exceptionally the thoracic muscles are affected, and produce more or less severe difficulty of breathing. The paralysis may occur after mild as well as severe cases of diphtheria. I have observed it even after ordinary sore throat, which had been of so little consequence as to have been almost overlooked, and when the paralysis really seemed to form almost the first sign of the diease. In some epidemics of diphtheria the resulting paralysis is frequently seen, whilst in others it is much more rare; it occurs in sucklings as well as in grown children, and in them, by preventing swallowing and breathing it becomes especially dangerous. In general, diphtheritic paralysis admits of a favorable prognosis, especially when it appears during convalescence from the disease. In all my own cases complete recovery has taken place in a few weeks or months.

The explanation of diphtheritic paralysis is still unknown, but it may be suggested in various ways, as diphtheria is regarded as a blood disease or not.

\section{Paralysis of 'Traumatic Origin}

Various traumatic influences, as blows, falls, contusions, and wounds, may excite complete or incomplete paralysis in children. 'T'o this category belong the paralyses in the regions of the facial nerve and brachial plexus in the new-born, produced by the pressure of the forceps, or by the sides of a narrow pelvis. Such a paralysis I have seen in the left arm of a six-year-old girl, which followed a severe blow and lasted four weeks, and Poyet records such another which lasted three months. Amongst these must also be classed paralysis occurring in one or both arms of young children, produced by rough handling or lifting up by the arms (Chassaignac and Poyet). These are not uncommon, and treated as contusions generally they disappear in a few days, and are due to straining of the nerves. Traumatic paralysis almost always admits of a favorable prognosis, and disappears in a few days-at the latest, perhaps, in a few weeks. 


\section{Rheumatic Paralysis}

This is, probably, only a rare occurrence in children, and is met with in the same forms as in adults. The influence of cold draughts of air on a heated body, or chilling by sitting on cold stones, may now and then excite such paralysis. Henoch and Romberg observed rheumatic facial paralysis in children between two and eight years of age, and I have myself seen it on the right side of a boy three years old, in whom it disappeared in three weeks.

\section{Paralysis from Mqterial Changes in the Central Nervous System and from Disease of Bone}

This has already been alluded to among the special diseases of the brain and spinal marrow. Here it may be enough to note that hemiplegia in childhood may frequently have its origin in cerebral tumours, especially of a tubercular kind, that apoplexy and encephalitis are more rarely the causes of paralysis than in the adult, and that these latter show themselves generally as hemiplegia, seldom as paraplegia. I have met with two cases of hemiplegia in which the paralysis occurred along with loss of consciousness in the midst of the best health. Postmortem examination showed apoplectic clots and partial encephalitis with atrophy of the brain-tissue. In one case of paraplegia, in a three-year-old child, after a course of eight months the whole of the paralysis became retrogressive; in a boy, aged four years, attacked by heart disease, left hemiplegia occurred suddenly along with loss of power of speech, yet in eight weeks he completely recovercd. Under this category niay also be placed all paralyses accompanying hydrocephalus, meningitis, atrophy, and sclerosis of the brain, intra-meningeal apoplexy, together with the diseases of the spinal cord, forming, as they do, only symptoms of the various diseases. Löschner has described a rare case of paralysis from sarcomatous growth in the spinal marrow. Facial paralysis is not uncommon from caries of the petrous bones, and paralysis from caries of the vertebræ is also met with. The former is often the 'result of chronic inflammation of 
the middle and inner ear, and is met with in scrofulous and tubercular children, and as the sequele of the acute exanthemata, especially of scarlet fever.

This affection, when the result of caries of the petrous bones, is almost always incurable, and I have met with no case where recovery has taken place. According as the destruction of the Fallopian canal and its contained nerve takes place above or below the giving off of the greater superficial petrosal nerve the uvula will show, or not, a tendency to one side, the point being drawn towards the paralysed side. In paralysis from disease of the vertebræ the limbs are affected according to the site of the disease, sometimes all, sometimes only the lower limbs being affected, whilst paralysis of the bladder and rectum are often also present. Such cases rarely recover, though such a fortunate result is not impossible.

\section{Paralysis Myo-sclerosica, the Pseudo-hypertrophic Muscular Paralysis of Duchenne}

This disease has been known only of late years, having been first exhaustively described by Duchenne. It is of rare occurrence, and the following observations are for the most part taken from those of Duchenne ('Journal für Kinderkrankheiten,' heft 586, 1868). It is characterised chiefly by weakening of the movements of the muscles of the legs and thighs, which gradually extends to the arms, and increases till all power of movement is destroyed; also by increase in volume of some of the attacked muscles, or, what is more rare, of almost the whole of the muscles of the affected parts ; finally, by irregular development (hyperplasia) of the interstitial connective tissue of the paralysed muscles, with proliferation of the fibrous tissue or the production of fatty granules in the advanced stage.

The disease begins either soon after birth or towards the sixth, seventh, or tenth year of age, and has chiefly the following symptoms:-A weakness of the legs exists, either from earliest infancy, or sets in later, and yet the muscles are well developed, and may subsequently attain to an unusual volume. In standing or walking they are remarkably bent, and there is a peculiar saddle-like curving-in of the lumbar region, and a waddling 
movement of the trunk in walking. The further course of the disease may occupy years, though it seldom remains stationary, the paralysis extending to the upper limbs and destroying completely the power of movement until death occurs by exhaustion and phthisis. Together with these phenomena cerebral symptoms sometimes occur, but not constantly, such as difficult and protracted articulation and a certain dulling of the intelligence, or even complete idiocy. Duchenne divides the course of the disease into periods, the first of which is indicated by the muscular weakness, the second by evident muscular hypertrophy, and the third by the pseudo-hypertrophic paralysis.

The pathogeny of the hypertrophic paralysis is obscure, for post-mortem examination has never discovered any perceptible alteration, either in the brain or spinal cord, by which the progressive muscular weakness can be explained. Neither can this peculiarity be explained by the compression or destruction of the muscular fibres by the increase of the interstitial connective tissue, since the muscular weakness occurs earlier than the hyperplasia, and there is no constant relation of proportion between them. A morbidly excited tendency to the formation of interstitial connective tissue alone seems to be the cause of the muscular weakness, though how this tendency is excited is a problem of which we are still without the key. The pseudohypertrophic paralysis is a disease of infancy, and appears, according to our present experience, more frequently in boys than in girls. We also find that several children in the same family may be attacked by it, and it is also probably hereditary.

The prognosis is never favorable, for Duchenne, in each case where the stage of hypertrophy was reached, had a fatal issue, though he believes recovery to be not impossible in the first period.

Treatment.-Aromatic spirituous liniments seem to be of service in the spinal palsy of children, especially if not of long standing, as the spirit of camphor and compound soap liniment, or the tincture of nux vomica, as employed by Heine. The latter is also said to be beneficial when given internally, though I cannot record any good results from it. If there is an absence of brain symptoms there should be no delay in the use of galvanism, especially the constant current, with the precaution, in 
young children, of not continuing the application more than from one to five minutes, and not more frequently than on alternate days. On the days when the galvanism is not applied I generally order baths containing the Franzenbad ferruginous salts, added in a concentrated form. Half a pound to a pound of these salts will suffice for a local or general bath for a child, and they are best used in the morning. By gymnastic movements and methodic kneading of the affected muscles their fatty degeneration and atrophy may be very greatly prevented. In deformities and persistent paralysis special mechanical apparatus is required. If there are indications of rickets, then the usual remedies-cod oil and iron with iodine-are to be administered.

For the treatment of diphtheritic paralysis quinine and iron, combined with good food and fresh air, stand before all other remedies; though, as a rule, the affection will get well of its own accord, and almost always, as has been already said, it has a fortunate result. Traumatic palsies likewise have need of no exceptional treatment; simple spirituous lotions, absorbent ointments, and repeated warm applications, as a rule, do all that is wanted. In one case I saw rapid recovery follow a course of the warm baths at Teplitz. Rheumatic paralysis almost always yields to electricity.

If the paralysis be due to cerebral hæmorrhage, then the practitioner must delay interference, especially during the early stage of the case, and only when all brain symptoms have disappeared can he begin a course of electrical treatment. If the brain lesion be serious it will defy all treatment.

For pseudo-hypertrophic paralysis Duchenne recommends the direct faradisation of the muscles, and the employment of the water-cure with methodic kneading of the muscles. As long as the first stage is not overstepped he regards recovery as possible. In the second stage no treatment has as yet been of any service.

In paralysis of the bladder and rectum great care must be taken to secure the regular emptying of the viscera by the catheter and clysters. 
(i) Arthrogryposis ; Contractura artium ; Essential contractions

By essential contractions is meant a peculiar and not frequent form of tonic painful convulsion of a limb, which is seen as morbid flexion, rarely extension, of the joints of the fingers, toes, the elbow, wrist and ankle. The fingers, strongly flexed, cover the inturned thumbs, forming a half-fist, and the hand also is so bent as almost to form a right angle with the forearm. The toes are generally flexed, and the foot contracted in the form of talipes varus. Along with these flexions sometimes, but rarely, there occur also contractions of the elbows and knees. The contractions are met with more frequently in the upper than in the lower limbs, not unfrequently in all the limbs at once; and they may occur suddenly, or be ushered in by or appear along with other symptoms, such as despondency, fractiousness, unquiet and broken sleep, vomiting, and painful cries. Their duration may extend over only a few hours, or some days or weeks; or they may be interrupted by intermissions of some length : other forms of convulsion may also alternate with them, as spasm of the glottis.

These tonic spasms of the limbs may be due to reflex action, and then they form a purely motor neurosis; or they may be symptomatic and indicative of a grave but insidious brain lesion. The causes of the former variety are still unknown, though dentition and cold may be indicated as such, and Henoch has added lithiasis renalis; while $I$ have frequently seen it in rickety children, and regard this condition as a predisposing cause. Children attacked by the contractions are generally exhausted by other diseases, especially by intestinal catarrh, though I have also met with them in well-nourished children. They occur most frequently between the first and third years of life, and when reflex they are generally harmless and sooner or later disappear. It is otherwise, however, when they are symptomatic and are due to a central lesion, such as internal hydrocephalus, as once observed by myself. In another case, worthy of notice, I met with the contractions of the hands and feet existing in a rickety girl aged nine months. 'They lasted 
from eight to fourteen days, then remission for four or five days occurred, and this went on for several months. Except a certain amount of restlessness at night and a somewhat exalted temperature and loss of appetite during the existence of the contractions, she had no symptoms; but violent convulsions came on suddenly, and afterwards paralysis, followed in two days by death. The section discovered considerable ventricular dropsy. (Pädiat. Mittheilungen von Steiner und Neuritter.)

Treatment.-When the contractions are reflex a careful consideration of the causes may justify the administration internally of the preparations of zinc, of opium, or of quinine: the external use of chloroform and the wrapping of the affected limbs in wadding may also be useful. In the symptomatic varieties drugs are not of much use, though any remedies may be employed which are looked on as suitable for the central lesion.

\section{Neurotic Atrophy of the Face}

This, a rare affection and but little known, consists of more or less marked unilateral atrophy of the face, while the unaffected side remains of normal size and appearance. The skin of the side on which the atrophy occurs is thin and stretched; the cushions of fat disappear, the muscles become thinned and the bones more delicate and smaller. The eye on the affected side is evidently smaller than its fellow, and more deeply sunk in the orbit, and the corresponding half of the tongue is smaller than the other, and the hair of the scalp shares in the want of symmetry. Sensibility and motor power generally remain undisturbed. The disease begins and extends uniformly over the whole half of the face, or it may appear as scattered patches, which, gradually increasing, finally extend over the whole half of the countenance. 'The atrophy occurs generally throughout the whole region supplied by the trigeminus; more rarely it is confined to the distribution of one of its branches. Of ten cases reported by Gerhardt there were eight girls and ten boys, and eight of the cases occurred in children between the first and the fifteenth years. The actual lesion on which this affection depends is yet unknown, and only few 
exciting causes have been suggested, such as burns of the face, blows producing effusion of blood, scrofulous glands of the neck, suppurative angina, and hooping-cough. All remedies have as yet proved valueless. 


\section{CHAPTER III}

\section{DISEASES OF THE ORGANS OF RESPIRATION}

\section{Asphyxia Neonatorum}

THrs term is applied to the condition of the child when, after its complete birth, respiration is not established, or established only imperfectly, and when at the same time it is doubtful if the heart is beating. In these cases the child is often well nourished, with a fat well-developed body, the face swollen and discoloured, the umbilical cord thick and distended, the mouth occupied with frothy mucus, the tongue drawn back against the palate or pressed between the gums, forming an instance of the asphyxia livida or hyperæmica of Kilian and Scanzoni; or, on the other hand, the child may be ill-developed, with pale and wrinkled skin, with loosely-hanging extremities and very weak action of the heart, instancing the asphyxia pallida or anæmica of other authors. We cannot, however, class all cases of asphyxia neonatorum under these two varieties.

The child lies motionless, and breathes hardly at all or very inefficiently, and is usually insensible to external irritations ; this condition lasting a short time unless some effectual assistance is rendered it, and then the defective attempts at respiration entirely cease, and the heart gradually ceases to beat.

The immediate cause of death in these cases is to be found in an overcharging of the blood with carbonic acid, as a result of which the irritability of the respiratory centre and also of the spinal cord is extinguished and the rhythmic performance of respiration rendered impossible, the resulting condition exhibiting all the appearances seen in asphyxia, though the term apnoa is more properly applicable to it.

Any lesions by which the admission of oxidized blood to the child is either impeded or altogether prevented may lead to 
asphyxia, and amongst them may be mentioned premature separation or disease of the placenta, compression, twisting or laceration of the cord, long-continued pressure on the head, or extensive intra-cranial hæmorrhage in the course of protracted labour.

Treatment.-The first and most important part of the treatment consists in the establishment of the respiratory movements in the spcediest way possible, and for this purpose the cord must be unravelled and divided as soon as the child is born, the cavities of the mouth and nostrils cleared of mucus, and attempts made to induce respiration. Of these perhaps the most effectual are slapping the chest with the hand, turning the child alternately on the side and back, raising the arms up and down, as in Sylvesterr's method of artificial respiration, and dashing cold water on the chest. Should these fail, faradisation may be tried in the course of the phrenic nerves in the neck, in order to induce action of the diaphragm, or insuffla. tion of the lungs, by blowing into the mouth directly, or by a catheter introduced into the trachea. In many of the less severe cases these means will succeed, but in the more marked cases frequently they will all fail.

\section{a.-Diseases of the Nasal Cavities}

\section{Catarrh; Coryza; Cold in the Head}

Catarrh of the nasal mucous membrane may be either acute or chronic, and may occur idiopathically as an independent affection, or symptomatically in the course of other diseases. It is characterised by swelling and increased redness of the mucous membrane, by increased secretion which is at first clear and watery, but afterward viscid and turbid, and by the irritant quality of this secretion there soon occur reddening and swelling of the nostrils and upper lip, with superficial excoriations and the formation of crusts. Nasal catarh may be accompanied by a severe amount of fever, and this is especially the case in the first few months of life, though it may also occur without any constitutional symptoms of pyrexia. It is 
frequently ushered in by severe general symptoms, as restlessness, crying as if in pain, snuffling and difficult breathing, disturbed sleep, even delirium, very hot skin, an exalted pulse of 140 to 160 , frequent sneezing and pain over the forehead, and finally increased secretion. It seldom remains confined to the nostrils, but spreads through the lachrymal passages to the conjunctiva, also downwards to the throat, larynx, and even to the deeper air passages, and by such extension produces difficulty of swallowing, hoarseness and barking cough. It may also pass along the Eustachian tube to the cavity of the ear, and there produce pain and tinnitus. In more advanced children nasal catarrh is an ailment free from much risk, but in those at the breast it is far from being free from anxiety; for in them respiration is mostly carried on through the nose, especially when feeding, and an interruption to the passage may seriously interfere with their nutrition, or even hazard suffocation.

Acute nasal catarrh readily becomes chronic, especially in scrofulous and syphilitic children. Prolonged duration of the disease may produce, by the decomposition of the discharge, ulcers, diphtheritic exudation, or even necrosis of some of the bones of the nostril. The intensely fetid odour emitted by patients suffering in this way enables the disease to be soon detected when they come near. It is usually very obstinate, often incurable, and always produces, sooner or later, impairment or complete destruction of the sense of smell.

Chills, very hot baths, inhalation of air charged with certain chemical or mechanical irritants, also some constitutional diseases, especially measles, may excite acute catarrh of the nostrils, whilst the chronic form is commonly kept up by scrofula, syphilis, new formations in, or polypous hypertrophy of the mucous membrane. Obstinate and frequently recurring nasal catarrh in sucklings should always excite suspicion of the existence of some specific taint, even when no other symptoms are present.

Treatment.-For the idiopathic nasal catarrh this is usually little more than confinement from the air in a chamber the temperature of which is kept equable, especially in the winter months, when the affection is often epidemic; baths are to be avoided, and care must be taken in the case of sucklings to obviate complete closure of the nostrils. For this purpose the 
incrustations on the nostrils ought to be frequently removed by a warm moist sponge, and sneezing excited by pressing a few drops of water into the nostrils. Nurses are in the habit of dropping milk into the nostrils of sucklings, to remove the inspissated mucus, and to smear the bridge of the nose with almond or olive oil. Astringent remedies, such as oxide of zinc, alum, or nitrate of silver, either in solution or in powder, may also be employed locally. In chronic coryza with irritating secretion and in ozæna the use of solutions of chloride of lime or permanganate of potash by the syringe or the use of snuffs containing alum or tannin, or of energetic cauterization with nitrate of silver, may prove beneficial. Guersant recommends as a snuff the following mixtures:-White precipitate, 1 part; powder of althea, 15 parts; or calomel, 2 parts, and powdered cinchona bark, 15 parts.

If the coryza is due to a scrofulous condition the usual treatment by cod oil, iodine, iodised mineral waters, iron, and iodide of iron, must be adopted; and if syphilis be suspected, mercury or iodide of potassium are the most likely means to be of service.

In the case of a suckling if the nostrils are so obstructed as to induce danger to life, milk either from the mother or from the cow, must be given by the spoon until the child is in a condition to take the breast.

\section{Epistaxis; Blenorrhagia; Bleeding at the Nose}

Epistaxis is rarely seen in newborn or suckling children, but in further advanced life, especially towards puberty, it is not an unusual phenomenon. The blood may flow only slowly in drops, or with such rapidity as to form a continuous stream, and it usually issues from one nostril only, rarely from both. If the flow is great in quantity, or if the child be seized while recumbent, the blood may pass into the pharynx, and be either spit out or swallowed, in which latter case it will be digested. The epistaxis is often preceded by such symptoms as headache, giddiness, scintillations of light before the eyes, or tinnitus aurium.

Causes.-These may sometimes be local, as blows, scratching 
the mucous membrane with the nails, ulcers, new formations, or diseases of the vessels of the mucous membrane ; or they may be general and due to some disproportionate blood-pressure. To this latter class belong the epistaxis seen at the commencement of pyrexial diseases, especially in the acute exanthemata, typhoid fever, diphtheria, parenchymatous nephritis, intermittent fever, in heart disease, hooping-cough, pneumonia, pleuritis, and empyema. As special causes of long-continuing epistaxis are sometimes found in children, hæmophilia or the hæmorrhagic diathesis, purpura simplex and hæmorrhagica, anæmia, chlorosis, and scrofula.

Epistaxis may be of very slight moment, or may be of serious import, according to the nature of its origin; as, for instance, it may be of positive benefit in the acute diseases, whilst in purpura and chlorosis it may be justly considered an element of danger.

Treatment.-A slight hæmorrhage from the nose, especially at the beginning of any acute disease, will seldom require any treatment, though $I$ have seen an epistaxis so severe at the onset of parenchymatous nephritis that it had to be arrested: If the hæmorrhage is very abundant, or is coincident with purpura hæmorrhagica, chlorosis, or scrofula, cold applications must be at once made to the forehead, nose and neck; inject cold water, or place a piece of ice into the bleeding nostril; or should this fail, apply the tampon, either with or without the addition of some astringent, such as alum, or sesquichloride of iron. After such local treatment of course it is necessary that the constitutional affection should be efficiently treated.

\section{New Formations in the Nose and Abscess of the Septum}

New formations in the nose in children are only seen in the form of polypi, and even these not frequently, as they are rarely met with before the sixth year.

If we follow the usual classification these polypi are either mucous, sometimes known as soft polypi, from their soft and delicate consistence; or they are formed of connective tissue in the form of fibrous or fibro-sarcomatous polypi, going some- 
times by the name of hard polypi. The former proceed from the mucous membrane, and are limited to it, whilst the fibrous polypi grow from the periosteum, frequently from the base of the skull, and gradually extend by growth in various directions, sometimes upwards to the floor of the orbit, backwards and downwards to the throat and larynx, or outwards to the cavity of the jaw, and they generally have a broad base.

Symptoms. - Nasal polypi do not produce much disturbance as long as they remain of moderate size; but when the increase of size is so great that the nostrils become plugged up, or if the growth takes any of the specific directions above alluded to, various symptoms are excited. Amongst these are irritation of the nasal mucous membrane with excoriations, hæmorrhage, loss of power of smell, a peculiar nasal tone of voice and breathing through the open mouth, interference with hearing, swallowing and chewing, lachrymation, difficulty of breathing, paroxysms of coughing, and frequent puffing and blowing to make a passage for the air. I had under my care at the Hospital for Children a girl six years old, who suffered from a fibro-sarcomatous, naso-pharyngeal polypus, which grew from the periosteum of the hard palate, closed the entrance to the posterior nares, and extended almost as far as the larynx, occupying the nasal and pharyngeal cavity as a rugged tumour, and pressing the epiglottis down on the chink of the glottis. In spite of several operations attempted for her relief the child died from the tumour, and on section I found similar growths in the lungs and glands of the neck.

Causes.-These are, for the most part, quite unknown. Even chronic nasal catarrh cannot be regarded as a cause, for in many cases it is not present, and in others it occurs only secondariì.

Diagnosis. - This may be somewhat doubtful at the beginning, but as the tumour grows mistakes become scarcely possible.

Treatment.-The only possible method of cure for these tumours is to remove them as early as possible. When they are not of large size, can be seen from the nostrils, and have narrow necks, they may easily be removed by forceps, or by entangling them in a snare, such as devised by Rosencrantz. When the pedicle can be reached by scissors Guersant recommends that method of removal. For the removal of deeply- 
rooted polypi serious surgical operations have sometimes to be performed, even resection of the upper jaw (Dupuytren, Robert, Lisfranc, Velpeau, and others).

Abscess of the septum comes occasionally, though not frequently, under observation, and may occur in an acute or insidious form, the latter especially in dyscrasic children. When the nostrils are examined, a flattish-round tumour of greater or less size will be observed, which fluctuates and is painful on the slightest touch, and the septum will be found displaced to one or other side. The pain and the obstruction to breathing makes the affection a very troublesome one. Sooner or later the abscess discharges spontaneously, though in scrofulous children this may be long delayed, and may be followed, as in one case which came under my notice, by perforation of the septum. The causes of these abscesses may be catarrhal, traumatic, or dyscrasic.

In the diagnosis care must be taken not to mistake the disease for polypus, but, as a rule, the pain will prevent any such confusion.

Treatment.-This consists of the application of poultices to the nose, the insertion of emollient substances into the nostrils, and the early evacuation of the abscess ; and when there is suspicion of some dyscrasia that must be treated, especially if there be any affection of the lones.

\section{B. Diseases of the Larynx}

\section{Laryngeal Catarrh and Pseudo-croup}

The infantile larynx is frequently the seat of disease, due to its smallness and delicacy and to its want of capacity for resisting external influences. Besides these physiological causes there scems to be, in certain individuals, families and even generations, a proclivity to laryngeal diseases, especially to laryngeal catarrh; and this is met with, not only in delicate children begotten by scrofulous parents, but also in children who are in every sense the opposite. Laryngeal catarrh occurs usually in the acute form-rarely in the chronic. The acute form is generally an idiopathic affection, but sometimes it 
is the indication of other diseases. Recent authors have attempted to separate laryngeal catarrh from pseudo-croup, but this seems to be only possible in so far as the latter is by preference a catarrhal affection of the rima glottidis, of the epiglottis and of the ligamenta ary-epiglottica, whilst in the catarrh proper the larynx itself is the seat of the disease.

The anatomical alteration in acute laryngeal catarrh consists in swelling and redness of the epiglottis and the ary-epiglottic ligaments, in streaky reddening of the vocal cords, with spots of extravasation and superficial denudation of the epithelium. The tonsils, uvula, and back of the throat also bear evidences of catarrhal affection, in the mucous membrane being swollen, dry, and red, with the papilla strongly marked, or it is covered with streaky mucus.

Symptoms.-Laryngeal catarrh occurs usually in a somewhat sudden manner in the midst of good health, or it may be superadded to an already existing nasal catarrh or some other affection of the more important air-passages. The child attacked may have been placed in bed apparently quite well, or affected only by a slight sneezing or coughing, yet may awake, after a few hours' sleep, with very alarming symptoms. When the patient is over two years old the symptoms by which the affection is marked consist of a ringing, croup-like cough, followed by a hoarse, grating and painful cry, difficult, panting breathing, an expression of anxiety and oppression, and frequent starting up in bed.

If seen soon after this first onset the patient's temperature will be found only slightly if at all exalted, the skin dry or slightly perspiring, especially on the head, the pulse somewhat accelerated, the breathing may be noiseless or accompanied by a hoarse rhonchus, the auxiliary muscles of respiration in action, the face slightly swollen, expressing anxiety, and the eyes staring. In many cases, after the first attack, there is nothing apparently the matter with the child, and the attendant may often have to wait for some time with the patient before the characteristic symptoms make their appearance.

After the first onset some time may elapse, during which the child lies placid, the breathing is easy and regular, the skin moist, and a moist rattling cough the only symptom, and the child may sleep quietly for some hours, until awakened by 
another croupal paroxysm. In the morning the child may appear to have nothing the matter with it beyond an ordinary catarrh, with slight moist cough and some hoarseness of voice. Frequently the disease ends with the one violent attack, though sometimes it may recur several nights in succession, as I have seen it do as many as eight times, or it may end in a true croup. Whilst acute laryngeal catarrh shows itself usually in the abovementioned fashion, between the first and third years of life, in some cases, and especially in the later periods of life, it is evinced only by hoarseness of voice, with a sense of dryness and soreness in the region of the larynx, an irritating cough, which is at first dry but afterwards moist, with expectoration of viscid purulent mucus, without any very great general disturbance, and without the alarming paroxysms.

Children who have once suffered from laryngeal catarrh may have it frequently recurring and from very slight causes, at least once or twice a year; and this disposition to its recurrence may last up to the serenth or as far as the twelfth year, by which time the larynx increases in proportional size and its irritability gradually decreases. Acute laryngeal catarrh cannot always at the onset be distinguished from true croup, though the absence of diphtheritic exudation patches in the pharynx, the normal condition of the lymphatic glands in the neck, the recession of the accompanying fever, the rapid issue in recovery, and the absence of the stenotic phenomena of the disease, will soon enable a correct diagnosis to be made. The recurrence of similar symptoms in one or more subsequent attacks further pronounces in favour of simple catarrh. The issue of acute laryngeal catarrh in recovery is the rule almost without exception, and it only occasionally happens that it advances into true exudative laryngitis, and then it assumes the well-known severe and dangerous characters of that fearful disease. Laryngeal catarrh often fastens on the deeper air-passages and becomes chronic.

Causes.-Exposure to cold, especially in cold dry weather, draughts, the inhalation of contaminated air, and over-straining through excessive crying, may be ranked amongst these. It is frequently an accompaniment of measles and hooping-cough, ulceration and new formations in the larynx, and is met with more rarely in smallpox, scarlet fever, and 
typhoid. It attacks children at all ages, most rarely sucklings, though I have met with it in children five or six months old.

Treatment.-The treatment required may sometimes be only tentative, at other times active medication may be needed. As soon as the symptoms begin warm milk, tea, or sugared water, should be freely given; and warm poultices, or compresses soaked in warm oil or hot water, should be applied to the throat. Should respiration be retarded or interfered with to a marked extent, or if there be doubt as to the severity of the disease, an emetic of small doses of tartar emetic should always be administered, provided there is no important contrary indication, accompanied by the application of such derivatives as sinapisms to the chest. Small doses of Dover's powder or extract of hyoscyamus are often useful in relieving the irritating cough; and when the latter is hard it may be much loosened by small doses of muriate of ammonia, given in the compound decoction of althea.

Should the catarrh become so severe as to give rise to symptoms of suffocation, though this does not often happen, emetics are useless and carbonic acid poisoning results, and tracheotomy is the only course left. The child must be kept in bed, or at least in the house, especially during winter time; as long as there is any trace of hoarseness left. To combat the resulting disposition there often is for recurrence of the catarrh, inhalation of such astringents as sal ammoniac, alum, and tannin, is to be recommended, together with bathing the neck and gargling the throat frequently with cold water. Care must be taken not to exclude the child too assiduously from the external air.

If the catarrh becomes chronic, inhalations of vapour containing astringents are advisable, and should there be any scrofulous or tubercular tendency appropriate treatment must be followed.

\section{Croup, Laryngitis crouposa vel Maligna, Exudative Croup}

Croup is an inflammation of the laryngeal mucous membrane, occurring generally in sporadic cases but sometimes epidemically, and on account of its great fatality it is a much dreaded disease of infancy.

Anatomy.-The varieties of disease classed under the name 
of croup do not always present the same pathological changes. The mucous membrane of the larynx is generally found covered by a tube-like or ragged layer of exudation, which may be partly adherent or quite loose, and of a yellowish-grey or brownish appearance, often completely occupying the canal of the trachea, this condition constituting true croup.

In diphtheritic croup the swollen and loosened mucous membrane, in parts pale and elsewhere intensely red, is scattered with spots of exudation, and at the same time with patches where the mucous membrane has been destroyed to a considerable depth. Purulent croup is a third variety in which the croupous membrane is entirely absent, its place being taken by a profuse purulent secretion, the epiglottis being more or less injected, its under surface covered with exudation, and in the sinuses of Morgagni there is almost always exudation-sometimes pus. The mucous membrane below is for the most part: deprived of its epithelium and here and there shows points of exudation, and the submucous tissue, especially about the aryepiglottic and glosso-epiglottic ligaments, is more or less œdematous. It may here be mentioned that the three varieties of croup just described cannot always be defined clinically, and may have exactly similar symptoms. After the occurrence of exudation in the larynx it often, though not always, extends into the pharynx and down the trachea into the bronchi. In two thirds of all the cases of croup under my care I have found the pharynx affected by diphtheritic exudation. Of fifty children examined after death from croup, in thirty-nine the croupous exudation was found to have extended more or less downwards from the larynx, in the form of tubes, cylinders, or isolated patches, with here and there ragged patches softened by suppuration. Further, there are often, though not constantly, such secondary complications as lobular, especially at the lower lobes, more rarely lobar pneumonia, gangrene of the lungs, pleuritic adhesions, ecchymosis of the visceral pleura, atelectasis, emphysema, passive hyperæmia of the brain and its coverings, hyperplasia and hyperæmia of the lymphatic glands of the neck under the lower jaw and along the trachea, swelling of the solitary glands of the intestinal mucous membrane and of the spleen, hyperæmia or catarrh of the kidneys, parenchymatous nephritis and spots of diphtheritic exudation on the skin or mucous tracts. 
Symptoms.-Croup rarely begins with its own peculiar and scvere symptoms, but is generally preceded by some catarrhal affection of the nose, throat, or larynx; and though these may last a few days, they are commonly enough overlooked by the relatives of the patient. Slight fever, frequent sneezing, altered disposition, broken sleep and dry cough constitute the usual premonitory symptoms, whilst the pronounced and important symptoms are the hoarseness of the voice and the dry ringing cough. The attack usually occurs during the night after the child has been asleep for a few hours, or towards morning, and from its commencement the hoarseness is persistent, or remits only for a short time, and generally passes into complete loss of voice so that the child can only whisper and the symptoms of obstruction become rapidly intensified. Frequent attacks of coughing, lasting a few seconds or minutes and producing congestion of the face, anxious straining of the eyes and distension of the veins of the neck and face, form a very distressing symptom. The breathing becomes difficult and gasping, and inspiration is accompanied by a loud rasping noise that may be sometimes heard at a considerable distance. The difficulty of breathing at first occurs spasmodically with intercurrent remissions, though later on it becomes continuous; all the accessory muscles of respiration are put in active employment; the cartilages of the nose may be seen in violent movement; the lips and cheeks become cyanotic; the muscles of the chest are strained to the utmost, and the diaphragm shows the boundary between the chest and abdomen by a marked hollow. The child is tormented by restlessness, tossing up and down, backwards and forwards, clutching at its surroundings and at its neck, sinking only for a few moments into a half-slumbering condition, soon again to awaken to its restlessness. This stage is more or less prolonged according to the rapidity of the case, but it usually passes into the condition of asphyxia in which are exhibited the indications of venous congestion of the brain and carbonic acid poisoning. The restlessness gives way to a condition of utter prostration and apathy; the face becomes of a white or leaden colour; the eyelids are motionless and half open and the eyes lose their lustre; the pulse becomes small, frequent, and intermittent; the skin cold and insensible; the breathing very superficial and frequent, and consciousness 
becomes rapidly clouded and finally disappears entirely. The painful scene is closed by coma, sometimes accompanied by trismus, contractions of the limbs or slight convulsions.

Should the disease have a favorable issue, which unfortunately does not often happen, the symptoms of obstruction will gradually disappear under the onset of a soft moist cough, the respirations becoming easier and the larynx clearer, a result that may be complete in six or eight days. The course of symptoms in the so-called ascending croup (aufsteigendem) is somewhat different, for there the symptoms of localisation of the disease in the larynx occur only after twelve or eighteen days, during which there lave been in existence indications of exudative bronchitis.

Examination of the cavities of the mouth and nose may reveal either only a slight reddening of the mucous membrane or exudative patches on the tonsils, uvula and fauces. In a few rare cases I have found the whole mucous membrane of the mouth and tongue covered by a croupous exudation, as in aphtha.

Laryngoscopic examination, should it be desirable for exact diagnosis, can be made only with great difficulty in little children-a difficulty which will of course be increased if there be any impediment to the breathing. Ziemssen, who has made such examination, found that the swollen vocal cords, covered with exudation, were quite fixed; that anteriorly their edges were directly in contact, and that posteriorly they were separated by a narrow cleft, which was broadest at the posterior commissure.

Physical examination of the chest does not always give trustworthy results, for the breath-sounds are sure to be more or less altered or muffled by the loud râles communicated from the larynx. Generally, the amount of air in the chest is diminished, and the sounds are, therefore, weaker, the more so if there be croupous bronchitis present. Atelectasis, due to condensation of the lung by lobar or lobular pneumonia, may sometimes, but not always, be determined by auscultation and percussion while the obstruction lasts; but if tracheotomy be performed and the hindrance to the entrance of air be thereby overcome, the condition of the chest may be more satisfactorily ascertained. In such a ease, if the croup be confined to the 
upper part of the air-passages, the vesicular murmur is moist and coarse ; but if the exudation have extended to the bronchi the sounds are weak, sometimes accompanied by râles, or when pneumonia is present the breathing may be bronchial in character.

The expectoration is either wholly absent from the commencement of the disease, or consists of white frothy mucous masses, and in the later stages either spontaneously or in the act of vomiting, copious masses of croupous membrane are ejected. These are often tubular or dendritic in form, and give clear indications of their origin. Along with these a copious ejection of mucus mixed with pus usually occurs. The croupous exudation is, however, very rapidly reproduced, and it happens sometimes that in the course of twenty-four hours two or more similar sheddings and ojections may occur. Getting rid of the exudation, either by coughing or vomiting it up, generally relieves the state of the patient, though unfortunately this relief is as a rule only temporary. The temperature curve shows very various relations, but none that are typical of the disease, for the temperature in one case may rise to $40^{\circ}$ or $41^{\circ}$ Cent., in another, scarcely less severe, it will not rise above $37.5^{\circ}$ or $38^{\circ}$. A continuously high temperature, especially when it persists after the performance of tracheotomy, is always indicative of an extension of the inflammation to the lungs or bronchi, and is, therefore, unfavorable for prognosis.

The urine frequently deposits a thick precipitate consisting of urates, or it may contain more or less albumen, according as there is presence or absence of some affection of the urinary organs.

The course of this disease is most frequently very acute, occupying little more than thirty-six to forty-eight hours, though sometimes it is subacute, and may be continued over from six to twenty-one days, the longest duration being in the ascending croup.

Should the disease have a fortunate issue there are many results and after-diseases to be looked for, as chronic hoarseness, a result of the swelling or cicatrization of the mucous membrane; complete loss of voice and difficulty of breathing with suffocative attacks, from cicatricial alteration or complete occlusion of the larynx; paralysis, anæmia, and dropsical affections, may be mentioned anong the sequelæ of the diphtheritic croup. 
Causes.-Infancy strongly predisposes to attacks of exudative croup, for it occurs most frequently in children between the second and seventh years of life, in children under two years of age only seldom, and sucklings are almost never attacked by it. Boys are affected more frequently than girls, for of eighty cases I have met with sixty-two in boys and eighteen in girls. True croup appears by preference in strong children, well-nourished, and previously healthy, and the diphtheritic variety without any apparent reference to constitution. A certain hereditary disposition to inflammatory croup exists in some without doubt. It occurs sporadically and in an epidemic form, idiopathically and as a result of exposure to cold, especially in the severe winter months, when the keen north and north-easterly winds are blowing; but it also occurs when the temperature is high. As a secondary disease, croup often occurs in the course of diphtheria, measles, scarlet fever, smallpox, and hooping-cough. True croup, as a rule, occurs in the same child only once, though there are a few occasional instances of a second attack. Some authors regard ordinary inflammatory croup as infectious, but with this I do not agree, though there can be no doubt that . the diphtheritic variety is eminently contagious.

Diagnosis.-It cannot be denied that at the onset true croup and pseudo-croup cannot be differentiated with certainty, though in the further course the increasing and continued fever, the recurring suffocative attacks, the indications of great disturbance, the ejected croupous membrane and tubes, the swollen submaxillary glands, and the presence of the diphtheritic exudation in the pharyux, are enough to set the diagnosis at rest. It must be remembered, however, that the absence of the exudation in the throat is no decisive argument against the croupous nature of the inflammation in the larynx.

Prognosis.-This is almost always dismal, a fatal result being almost the rule, for in tracheotomy alone there seems any chance of recovery. In the Children's Hospital at Prague 34.6 per cent. of the affected children have been saved by the operation. Complications of the laryngitis with tracheo-bronchitis and pneumonia make the prognosis, of course, still more unfavorable. The younger the child the less the chance of recovery, and the fatality of the disease is often increased by epidemic influences.

Treatment.-The indications for treatment in a case of croup 
are, firstly, to cut it short and prevent the occurrence of exudation if possible; to favour the removal of the exudation, if it has formed ; and, thirdly, to obviate the tendency to suffocation. For these purposes innumerable remedies have been recommended and again deserted; and scarce a year occurs in which something new is not advanced, yet, amidst them all, we have none in which any great confidence can be placed.

If the disease begins with croupo-diphtheritic pharyngitis, then the employment of remedies by inhalation, spray, or in older children by gargles, such as lime water, lactic acid, chlorate of potash, sesquichloride of iron, flowers of sulphur, nitrate of silver, carbolic acid, \&c., ought to be tried, though of all these the most useful, in my experience, has been lime-water. Abstraction of blood and methodic treatment on hydropathic principles do not seem to have any advantage over the other plans; the former is being more and more given up, while the latter, at least, makes the patient's condition somewhat easier, if there is no indication of a more directly favorable method of treatment, though the lowering of the patient's temperature seems to have no influence on the disease.

When urgent symptoms of laryngeal obstruction occur the best remedies are emetics, after the action of which there is almost always an improvement in the condition of the child, though, unfortunately, they do not always prevent the subsequent exudation. Ipecacuanha is now generally preferred to tartar emetic on account of the depression following the use of the latter, the dose of the former being from twenty to thirty grains in two tablespoonfuls of water. Sulphate of copper and sulphate of zinc, in six- or eight-grain doses, are also used as emetics. After free emesis has occurred two or three times the emetics may be discontinued, and demulcent drinks, as almond milk or marshmallow tea, given freely, and hot applications of poultices or fomentations should be made to the neck, or an ointment consisting of mercury, digitalis, and belladonna, rubbed over the front of the throat.

The emetics must be repeated on the recurrence of any suffocative attack as long as they seem to afford any relief. As the emetics lose their effect the indications of threatening asphyxia become more and more apparent, and the necessity for tracheotomy is established. This operation constitutes of itself no 
remedy against the croup, but, by giving nature time to bring about more favorable conditions, it is often of great value; and the sooner it is undertaken the more likely it is to be successful, though it ought not to be performed prematurely, or until the above-mentioned indications render it imperative.

The older the child is the less extensive will be the exudation, and the greater consequent probability of recovery after tracheotomy. In children under two years of age unfavorable results are increased by the occurrence of croupous bronchitis, broncho- or lobar-pneumonia, though these chances ought not to stand in the way of the performance of the operation. In the Children's Hospital at Prague ninety cases have been operated on, and of these, as already mentioned, $34 \cdot 6$ per cent. have recovered.

The instruments required for the operation are, a bistoury, several forceps, a director, and the proper tube ; a trustworthy assistant at the time of the operation and a trained nurse for the after-treatment, are also essential. On a careful attendance to the dietetic, surgical, and mechanical details of the aftertreatment depends in great measure the success of the operation. Continuance of the difficulty of breathing, febrile excitement and restlessness after the operation, are unfavorable signs and indicate an extension of the exudation down the air-passages below the seat of the operation.

Should the parents object to the operation, strong stimulant remedies, as wine, æther, \&c., must be given during the stage of asphyxia.

Loiseau and Bouchut have lately recommended catheterising the larynx by means of a tube introduced through the chink of the glottis, and Weinlechner has proposed medication of the interior of the air-passages as a prelude to tracheotomy.

(As Dr. Steiner has given no directions for the after-treatment of cases of tracheotomy, I may be forgiven if I interpolate here the results of my own experience of such cases, which has been pretty large.-L. 'T.)

Perhaps no surgical operation depends so much as tracheotomy on constant watchfulness and judicious management of the aftertreatment for its ultimate success; and the fact that in the majority of instances the operation has to be performed in an emergency and under very varying circumstances, the details of the 
management of each case must depend very much on the discrimination and judgment of the attendant practitioner. One great good which has been achieved by the general establishment of children's hospitals in our large towns is that we have in them the means of training nurses for the special management of cases of tracheotomy, so that when the operation is needed in private practice the surgeon knows that an assistant is within reach to whom a large share of the responsibility of the case may be trusted, and who will effectually guard against some of the accidents to which the operation is liable. Such a trained nurse should always be secured, if possible, especially when the patient is a child.

The details of the operation may here be omitted, though a few words on the apparatus required for its performance are necessary, as they continue to be required in the after-treatment of the case. First in importance is the tube, and that which I prefer is the old-fashioned double canula with a cleft in the outer tube; for I have a rooted aversion to complicated instruments. The lobster-tail tube is very ingenious, but has no compensating advantage for its increased expense; and it is, moreover, very liable to be out of order when most needed. Eren the double canula will sometimes be found too much, and recourse to the single solid tube will be found necessary. Whatcver tube be employed great care is necessary to see that the tubes are well made. I have known two cases where the shield has become detached and the tube has lodged in the trachea. At least two fresh tubes should always be in readiness for the purpose of change, which is frequently necessary, and the one removed should be placed at once in a strong brine and there left to soak for some hours, being subsequently well cleansed and oiled. The nurse should be competent to change the inner tube when necessary, and have an abundant supply of duck-wing feathers by which to clean it. A pair of slightly curved dressing forceps, some lint and tape, are all the further requirements, though it is as well to leave at the patient's house such instruments as may be necessary for the re-insertion of the tube in case of its being coughed out.

If the single tube is used there is much more trouble in keeping it patent, as the feathers must be passed to clear it of mucus whilst it is in the wound. The inevitable result of this 
is that every time the feather is inserted an attack of coughing is induced, which harasses the patient and aggravates the lung complications.

In adults the operation has a very much larger success than it has in children, and it is also a much rarer operation, owing to the differences in the conditions which necessitate it. It is seldom had recourse to in children for any diseases other than diphtheria and croup, and on this account the resulting mortality is very high, the after complications being numerous and serious. The first of these is the extension of the obstructive exudation below the artificial opening in the trachea, a complication which may actually have been present at the time of operation, or which may have shortly followed it. I need hardly say that this is almost always a fatal state of matters, and that cases, where the false membrane is loosened and coughed-up and recovery results, are very uncommon. Should evidences of such an effort occur, the nurse must be directed to remove the inner tube when a severe attack occurs, and to be prepared to remove with the forceps any tag of membrane that may appear in the tube. Cases are recorded where the loosened membrane has been fished for down the trachea, but this must be undertaken by the surgeon alone.

The state of the room in which the patient is kept must be carefully attended to, and the most important point is undoubtedly to secure that the temperature shall remain equable. For this purpose a wet and dry bulb thermometer should be kept in the room, and the moisture of its atmosphere carefully regulated by the admission of steam from the nozzle of a kettle. Nothing, however, can be more detrimental to a child who has had tracheotomy performed, and who possibly has incipient broncho-pneumonia, than to have to breathe an atmosphere super-saturated with steam, oppressive almost to a healthy adult, as I have often had occasion to notice. It is sufficient to guard against great dryness and any marked variation of the temperature of the room. The child must be warmly clad, and two or three folds of light muslin arranged so as to cover but not occlude the orifice of the tube, and to act like a respirator, for the purpose of reducing the conditions of the inspired air as nearly as possible to those of that which is expired, as far as temperature and moisture are concerned. 
For the first twenty-four hours after the operation the patient does not give much trouble, for the tube is not at that time apt to get choked up and, unless the case is one where the operation has been delayed to the last, the complications have not yet become established. After that period bronchitis and pneumonia, the direct results of the operation, are the complications to be dreaded, and to them is due probably seventy-five per cent. of the mortality of the cases which survive the primary dangers of the extension of the croupous or diphtheritic exudation down the air-passages. Death from this latter cause usually occurs within a very few hours after the operation. Should bronchitis or pneumonia, or both, supervene, there are two methods of treatment that have proved of service in my experience, the free administration of stimulants and the liberal application of hot poultices to the chest. The former are best given in some form of wine whey, and that which children like best is made by cracking a pint of new milk with a glass of champagne, boiling it for a moment and straining off the curd. The addition of a few drops of sal-volatile to this seems to aid its digestion. An application of the poultices requires both intelligence and perseverance, for if once begun they cannot with safety be discontinued until the crisis has been passed. 'They must be made like jackets, to encompass the back, sides and front of the chest, must be changed every three or four hours, and the old poultice must not be removed until the fresh one is quite ready for application. Their proper use may therefore be seen to be troublesome, but unless implicitly carried out it had better never be begun. I do not think that any drugs should be administered in these cases, especially in very young children, for the all-important consideration is the maintenance of gastric digestion, and the routine administration of emetics is more likely to destroy this than anything I can think of.

If the case progresses favorably for a few days another source of danger presents itself, a direct result of the operation. I refer to the passage of the food through the chink of the glottis and the production of violent attacks of coughing on the slightest attempt to swallow. As this complication becomes more marked the food is seen to be ejected from the tube. I have seen several most promising cases succumb to the constant irri- 
tation and to the starvation consequent on this most disappointing accident. What to do under the circumstances I am at a loss to recommend. They are certainly due to paralysis of the muscles closing the glottis, and on this ground $I$ have tried galvanism, but without result.

A few cases run the gauntlet of all these complications, and when such have reached the third or fourth week they may be reckoned as recoveries, the only care left being to decide when the tube can be dispensed with. In some this happy time never arises, and they go through life wearing a series of tubes. In the majority of cases, however, a time does arrive when the natural passage for air becomes again patent, though this is often long delayed. In one of my successful cases the patient could not get any air through the larynx for nearly four months after an operation which had been performed for croup occurring after measles. When convalescence has become fairly established trials may be made day after day by keeping out the inner tube and occluding the orifice of the outer tube for a few seconds till the possibility of laryngeal inspiration has been positively ascertained. 'This may be further and further encouraged by closing the orifice of the tube for an increasingly long time, but the tube must on no account be left out for any period longer than a few minutes as long as there is any chance of its being again required. The rapidity with which a tracheotomy wound will close when the tube is left out is almost incredible, and I have more than once known a surgeon go back in half an hour, or some such time, to replace a tube which he had left out, only to find that he had either to leave it out or enlarge the wound. When the tube can be dispensed with the wound generally heals immediately, though on one occasion I have had to pare the edges of a tracheotomy wound fourteen years old and close it, its previous occlusion having been accomplished by the patient himself using a plug of moistened paper. One of these plugs got into the trachea, and I had to fish it out, and then I closed the wound. 


\section{Tumours of the Larynx}

These are seldom met with in childhood, and among the cases that have been noticed, papillomatous growths take the most prominent position, as being almost exclusive of all other kinds. I have seen four cases of laryngeal tumours, all papillomatous. If the new formation has reached any size, it presents a pinkishwhite cauliflower or mulberry appearance, more or less soft and friable when pressed between the fingers, and under the microscope is seen to be a hypertrophy of the mucous membrane and subjacent connective tissue. The seats and sizes of these growths vary very much, the anterior half of the edges of the vocal cords being the faworite positions, though they may be formed all over these structures, and may extend to the neighbouring mucous membrane. Lobular pneumonia, atelectasis and marginal emphysema and croup of the trachea and bronchi, are also found to occur along with them.

Symptoms.-The first symptom is usually hoarseness, which may be, according to the seat of the disease, transient, or obstinately persistent and increasing gradually until there is complete loss of roice, so that the child can do little more than whisper. When the tumour has its origin at some distance from the vocal cords, there may be no hoarseness for some time after the tumour has been in existence. Usually with the appearance of the hoarseness there is also some difficulty of breathing, though the occurrence of this important symptom is sometimes delayed. This latter condition gradually increases in severity until distinct suffocative attacks occur, especially after any unusual effort at speaking or crying, after any catarrhal attack, and frequently during sleep, owing probably to the recumbent position.

The hoarseness and the obstruction to the breathing increase as the tumour grows, until, as in croup, suffocation is threatened. There are no febrile symptoms at the beginning or throughout the course of the disease, though towards the end the temperature and pulse may rise somewhat.

The course of the disease is sometimes rapid, sometimes more prolonged, varying from a few months to some years; and after the tumour has been removed it is apt to recur. 
The essential causes of these tumours are quite unknown; as congenital affections they are rarely observed, and are more frequently developed during the later years of childhood. Boys are more subject to them than girls, and amongst exciting causes have been mentioned catarrh, croup, hooping-cough, the acute exanthemata especially measles, though these opinions must be accepted with reservation.

Diagnosis.-This can be made with precision by means of the laryngoscope in more advanced children after a little careful training, but in children under three years of age it is impracticable. The diagnosis may be made, however, with great probability, if the above-described symptoms are present, especially the chronic feverless course, the hoarseness, the difficulty of breathing, and finally the steady increase of the latter, accompanied by symptoms of laryngeal stenosis, these preventing any confusion between this affection and simple chronic catarrh, croup, or paralysis of the cords. The most probable difficulty is that of distinguishing between it and syphilitic or tubercular disease of the larynx; but a careful investigation into the history and the discovery of some other evidence of onc or other of these conditions would at once determine the case, with an ultimate appeal to the laryngoscope if its use were at all practicable.

Prognosis. - The results recorded in Gerhardt's 'Manual of Children's Diseases' afford a clue to the prognosis of these cases. : Of fifty-two cases of laryngeal tumour under fifteen years of age, thirty died, six having had tracheotomy performed; in seventeen the disease recurred, after operations had been effected; in two others the tumours were partially removed and the patients improved. The remaining three are also returned as relieved. Of the four cases which have come under my own notice, one was a boy six years old in whom an attack of croup occurred after tracheotomy and removal of the tumour, yet the case resulted in recovery, and four years after the operation there were no signs of a return of the growths. Two died of asphyxia after tracheotomy had been declined, and one was removed from the hospital unrelieved.

Treatment.-The only course is to remove the growths by operative measures, unless the child be too young, or there be some other reason against such a measure. For this purpose tracheotomy is almost always necessary, especially when there 
is severe laryngeal stenosis. Children under three years of age are hardly fit subjects for such an operation. The removal of the tumour by access from the mouth seems to give better results, according to the experience of Braimer, Ehrmann, Burow, Balossa and others, than the laying open the box of the larynx in the middle line and thus removing the growths. Pediculated tumours are most easily removed by means of a snare, while those with a broad base are recommended by Bruno to be removed by scraping with instruments adapted for the purpose. It must not be forgotten that the most perfect method of operation does not exclude the possibility of return.

\section{Spasm of the Glottis}

This affection belongs almost exclusively to infancy, and is due to the narrowness and delicacy of the chink of the glottis, and to the extreme irritability of the nervous system during infancy. -

Anatomy.-In spite of its frequent occurrence the pathological basis of this disease is yet but little known, for in its xtiology there are mysterious influences which are still beyond our powers of investigation. In a number of sections I have found various conditions, as acute and chronic hydrocephalus, anæmia and hyperæmia of the brain, more or less pronounced rickety condition of the skull with or without craniotabes or rickets of other parts of the skeleton, and cheesy hypertrophy of the bronchial glands. The thymus gland was almost always already absorbed, and could not in any case be regarded as the exciting cause of the spasm.

Symptoms and course.-Children attacked by this affection are almost always more or less anæmic, though they may be either apparently healthy, with good cushions of fat, or they may be not so well nourished. The spasm usually occurs suddenly and with but little warning, being generally preceded only by some restlessness and a few cries. The face becomes red or reddishblue, with a marked ring round the lips; the eyes stare and evince great suffering; the head is bent backwards; the extrenities may either be in active motion, may hang helplessly 
down, or be spasmodically stiffened; the upper limbs even may be in a condition of contraction. The temperature of the skin falls, especially of the trunk and lower extremities. The essential peculiarity of the affection consists in painful efforts on the part of the child to make an inspiration, gaspings for breath, accompanied by a peculiar crowing or piping noise. Now and then, at intervals of some seconds or even a minute or two, these efforts succeed in effecting an inspiration accompanied by a loud crowing noise. Consciousness is not usually interfered with, though it may be disturbed transiently to some extent, or even wholly. After the attack is over the breathing and appearance gradually become normal, and remain so until another onset of the spasm.

The frequency of the attacks varies very much, from two or three in twenty-four hours to as many as sixty in the same time. In some cases we see a marked increase and gradual recession in the frequency of the attacks. A very important fact in the xtiology of the disease is moreover seen in the fact that the glottic spasm is sometimes-probably in half the cases-preceded by or alternates with a marked epileptic attack. The cases often end in hydrocephalic effusion, with general convulsions and symptoms of severe brain pressure, whilst the laryngeal spasms become gradually less frequent in their occurrence, and finally entirely disappear.

The duration of this affection is various in its length. In a few cases death occurs during the first attack, as I have twice observed in rickety children between six and eight months old, who died suddenly of suffocation while being bathed. The disease usually lasts a few weeks or months, having pauses occasionally of considerable duration, after which it reappears, sometimes in a milder, sometimes in a more severe form. As a rule, the disposition to this form of spasm and its severity diminish with the increasing age of the child. Frequency sometimes seems to give a relatively slight form to the attacks, even so that it may hardly have the character of a disease, and may be left alone without much risk; though, on the other hand, the spasms may remain so severe as seriously to endanger the child's life, or at least to very materially interfere with its growth and nutrition. 
According to the statements of most authors recovery is the less common result of the disease.

Causes.-The essential process of the affection is a transient spasmodic contraction of the arytenoid muscles, the direct or indirect origin of which is to be sought for in an irritation of the respiratory centre of the medulla oblongata. This irritation may arise, as pointed out by Hensch, either centrally or in some reflex manner.

The most frequent origin is undoubtedly in some disturbance of the nutrition of the nerve substance itself, leading to irritation of the medulla oblongata; and this is very frequently due to anæmia, hyperæmia, or serous effusion, especially in ricketty children. I have also found that the majority of the patients have shown indications of rickets, and there evidently exists some causal connection between the two diseases more or less intimate, though nothing definite can yet be asserted about it. Craniotabes may often be seen in coexistence with spasm of the glottis, but it does not seem to have much importance, for the latter is often seen without it. We must regard faulty nutrition of the brain as playing a very important part in the production of this disease, and also, probably, the extreme irritability of the brain during the first two years of life.

Spasm of the glottis, when excited by reflex action, is generally due to some such condition as catarrh of the throat and primary air-passages and the introduction into them of foreign bodies, irritation of the mucous membrane of the larynx, hooping-cough, cheesy and tubercular disease of the bronchial glands, over-straining of the larynx from violent crying. Sometimes it may occur as a result of teething, from irritation of the intestinal nerves in intestinal catarrh, accumulation of faces or the presence of worms. Enlargement of the thymus gland and patency of any of the structures peculiar to the foctal circulation, may be struck out of the list of causes.

As predisposing causes must be mentioned age, sex and rickets. Of 226 cases which have come under my own notice, 174 were in the first year of life and the remaining 52 between the second and third years; and the relative proportion of the sexes was 150 boys to 76 girls. In both parents and children there was very frequent evidence of rickets.

Treatment.- This may be divided into two kinds; first, that 
by which the initial causes of the disease are to be combated; and secondly, that by which the spasmodic attacks themselves are to be alleviated. To the former belongs that which has its indications in a rickety, anæmic, or ill-developed condition of the child, consisting of iron and quinine, cod oil, fresh air and general attention to its dietetic treatment, together with mineral, malt or sea baths. Of the latter class oxide of zinc has seemed to me to be the most valuable agent, in doses of a half or one third of a grain given two or three times a day, especially when the laryngeal spasms are accompanied by other convulsions. I cannot say much in favour of musk, which has been much praised by Salathi.

When the attack occurs the fastenings of the child's clothes must at once be loosened, the head raised and cold water dashed on the face and chest and fresh air freely admitted to the patient. If the attack last long, counter-irritation of some kind, as a sinapism, may be applied to the breast or thighs. Most frequently, however, the attack is over before much can be done. Warm pillows stuffed with feathers should be removed and others filled with horsehair or sea-grass substituted for them.

\section{Paralysis of the Glottis}

If we are to depend on present statistics, this affection would seem to be uncommon in childhood, but it may be assumed that as we acquire a more certain knowledge of the lesion, the frequency of its occurrence will be more noticed.

Its symptoms consist of a group affecting the voice, as hoarseness or complete loss of voice, and another affecting respiration and consisting of obstructed breathing with obstinate and sometimes even convulsive attacks of coughing, accompanied by a peculiar, shrill, brassy sound which has a remote resemblance to the sound heard in croup. The disease has generally a very chronic course, though sometimes it may be acute, and this is determined by the nature of its exciting cause. Sometimes there are intermissions when the voice is clear and sonorous, due to some powerful mental or some external influence. After the paralysis has lasted some time there-results fatty degeneration and atrophy of the affected muscles, which may be any of those 
in the larynx, all being supplied by the superior and inferior or recurrent laryngeal branches of the vagus. If a laryngoscopic examination be made the larynx is found normal, but there is deficient and assymmetrical movement of the cords, the unequal tension of which produces the hoarseness. The affection is usually unaccompanied by any pyrexial symptoms.

The following varieties of the glottic paralysis may be classed according to the causes of their production:

I. That produced by lesion of the central nervous system, in such diseases as hydrocephalus, tubercular meningitis and tumours which, contracting the space occupied by the brain substance, produce pressure on the recurrent fibres.

II. That due to peripheral causes, as pressure by enlarged or tubercular glands in the teck on the vagus nerve or its recurrent branch.

III. That which is the result of local, catarrhal, or inflammatory affection in the larynx, and to this variety there belong the cases of paralysis of the cords after laryngeal catarrh, diphtheria and those due, according to Gerhardt, to idiopathic affection of the muscles.

IV. A reflex variety, due to the disturbances of puberty and, according to some authors, sometimes to the irritation of worms. I have seen in two cases of girls between eleven and twelve years of age, intermittent aphonia last until the occurrence of the menses, for eighteen months in one case and twenty in the other. This affection is, of course, more common in girls than in boys, and generally occurs between the tenth and fourteenth years of life.

Diagnosis. - This may be established with certainty by laryngoscopic examination, if it can be made; when this is impossible the intermittent character of the aphonia, the presence of some probable cause for it, such as large scrofulous glands in the neck, together with the previous occurrence of an attack of diphtheria within a recent period, and the obliquity of the epiglottis, will reveal the nature of the affection. An accurate diagnosis between this disease and chronic catarrh or a tumour of the larynx can, however, be made only by means of the laryngoscope.

Treatment.-This may be either causal or symptomatic. In the former case the treatment will consist of the administration 
of iron, iodine, and cod-liver oil, in cases where the presence of scrofulous glands in the neck is suspected to be the cause of the disease ; of quinine and iron when it has followed a diphtheritic attack; and of iron chiefly where it is due to the distress incident to the occurrence of puberty or to general anæmia. Enlarged glands may be removed from the neck by operative measures, or when this is not deemed advisable they may be locally treated by applications of the tincture of iron. Bruns has recently recommended a course of vocal gymnastics as a curative measure, this consisting of a regular exercise of the larynx by the articulation of the vowels alone at first, expressed as loudly as possible, and afterwards in combination with consonants. Electrotherapeutics have also found very general favour in this disease applied by the use of the interrupted, or, hetter still, the con: tinuous stream directly to the interior of the larynx by means of Morell Mackenzie's laryngeal galvaniser, the other pole being carried over the course of the vagus nerve. In children the direct application of one of the poles to the larynx is not feasible, and the current can be used only externally.

\section{Foreign Bodies in the Air-passages}

These may have an origin external to the economy, in that case consisting of buttons, beads, fruit-stones, or indeed, 'almost-any small substance that a child may put in its mouth; or they may come from some internal source, being then usually lumbrical worms, blood or pus from abscesses in the neck or pharynx, or cheesy masses detached from tubercular disease in the lungs or bronchial glands.

Symptoms.-These will vary according to the nature of the foreign body, according as it is fluid or solid, hard or soft, round or angular, large or small, \&c., giving rise to more or less distress, in difficulty of breathing, and paroxysms of coughing. A sharp angular substance may become fixed in the larynx or in a bronchus, and producing more or less complete obstruction of an airpassage, leading to serious symptoms; while a round substance, moving up and down with the respirations or with the cough, may produce but little disturbance. In the former case ulceration and suppuration are likely results, leading sometimes to perforation of 
the air-passages and wandering of the foreign body. Emphysema with rupture of lung-tissue, atelectasis and pneumonia, frequently lead to a fatal issue in cases where any foreign body has gained admission to the air-passages. In one very interesting case of a child on whom tracheotomy had been performed on account of croup, I found a lumbrical worm several inches long in the trachea and right bronchus, and the lung-tissue in the immediate neighbourhood of the bronchus was in a state of pneumonic infiltration.

If the foreign body be not removed death occurs sooner or later, either from suffocation or from the subsequent affections of the lungs and air-passages.

Prognosis.-This is always doubtful, and depends in a great measure on the size and character of the foreign body and on the age of the child.

Treatment.-Attempts may be made to remove the foreign body by exciting fits of coughing, or by turning the child upside down, shaking him, and striking him with the flat hand on the back. Together with these manœuvres emetics and stimulatives may be tried, though, if the foreign substance be sharp or angular, they may do more harm than good.

Should these means prove fruitless, and should symptoms of suffocation, restlessness, and cyanosis make their appearance, tracheotomy must be performed without delay, and the foreign body sought for and removed, if possible, by means of forceps. Should the operation not be permitted or be unsuccessful, any further treatment can be only symptomatic.

\section{c.-Diseases of the Trachea}

Diseases of the trachea are seldom primarily confined to this part of the air-passages, but more usually form part of a general catarrhal or inflammatory affection of the respiratory mucous membrane, as in catarrh, croup, and diphtheria. The symptoms are similar to those of the general affection, and have no in. dications for special treatment. Sometimes there are seen cases of specific affections of the trachea in children affected by syphilis, as recorded by Gerhardt, and observed by myself in a boy twelve years old. This takes the form of superficial or deep ulcera- 
tion with destruction of some of the rings, and subsequent diminution of the calibre of the tube.

Polypi of the tracheal mucous membrane are sometimes met with, producing the ordinary symptoms of obstruction. Sudden suffocation may occur in these cases, as noticed by Lieutaud, by impaction of the polypi between the rocal cords.

Thinning, ulceration, and complete perforation of the tube of the trachea, to a greater or less extent, may be the result of pressure of hyperplastic, inflamed, or tubercular glands in the neck, the seat of these alterations being usually the posterior or lateral walls; with reference to this accident it may be mentioned that obstruction of the larynx or of a bronchus or fatal pneumonia has been met with as the result of the drawing into the trachea of fragments of these glands. Such cases have been recorded by Löschner, Neureutter, and myself.

Besides stenosis being produced by alterations on the internal surface of the trachea, it may be the result of pressure from without, as from goitre, enlarged lymphatic glands, sarcoma of the anterior-region of the neck, as in a case which came under my own notice in which both the larynx and the upper part of the trachea were flattened, and during life certain symptoms of obstruction existed which lasted up till death.

Several authors also mention enlargement of the thymus as a cause of compression of the trachea.

Symptoms.-These consist in difficulty of breathing, with gasping, audible inspiratory efforts which are increased in the horizontal posture, cyanosis of the face and mucous membranes, small pulse, and clear sonorous voice if the larynx be not implicated. A careful examination into the history, the very chronic nature of the disease, and the existence of some such condition as syphilis, scrofula, enlarged glands, or goitre, will make the case clear.

Treatment.-This must always be governed by the cause of the pressure, and, if possible, the proper steps must be taken for its removal. 


\section{D.-Diseases of the Thyroid Body}

If we except the instance of goitre, the thyroid body is rarely the seat of any pathological change in infancy, A few cases have been recorded of traumatic inflammation affecting it, also cases of tubercle as I have myself observed, and, according to Demme, of cancer.

\section{Goitre}

This is seldom congenital, and does not occur so frequently in childhood as in adult life. It is met with most frequently in the endemic form in the goitrous districts.

The anatomical alteration consists usually of a simple hyperplasia of the physiological elements of the gland, the goitre being then a uniform tumour (struma lymphatica), or it may consist of several colloid masses or cysts of various sizes (struma cystica). The increase, in size may affect the whole thyroid or only part of it, forming the peculiar tumour which is sometimes regular and symmetrical, sometimes knotty and affecting only one or other lobe of the gland. Calcareous degeneration of the thyroid has been occasionally noticed.

Symptoms.-Congenital goitre has usually an unfavorable signification for the affected child, which is usually born apparently dead and can be made to breathe only with difficulty. It will not take the breast, the breathing becomes noisy and insufficient, the crics weaker and weaker, and it usually dies very soon after birth. Should it live for any time the symptoms already mentioned under stenosis of the trachea become pronounced, and atelectasis, broncho-pneumonia, œdema of the lungs, congestion of the venous system, with hyperæmia and œedema of the brain (Bednar) may be found after death. The child thrives only when the tumour is small. I saw a child with a goitre, born of a mother likewise affected to a high degree, which died asphyxiated in half an hour. The same mother had had another goitrous child two years previously, which lived fifteen days. In both cases the labours were severe. Therc were five other children, none of whom exhibited any 
affection of the thyroid except the eldest daughter, in whom the gland became markedly increased in size after eight years of age.

As an acquired disease goitre is usually developed between the periods of the second dentition and puberty. Girls are more frequently affected by it than boys, and if it occurs in a goitrous country it will frequently be found that either in the father or mother or in the grandparents goitre has existed. In certain districts the disease is very common. Acquired goitre is usually only a disfiguring and troublesome affection, the symptoms which accompany the congenital form and which are destructive to life, being rarely met with when the disease is developed within the period of childhood. I have seen one child, ten years old, die from the obstruction produced by a very large goitre.

Treatment.-In congenital goitre everything must be done which can possibly obviate the tendency to asphyxia and aid the nutrition of the child. Preparations of iodine may be tried for the reduction of the tumour, though they are not likely to have much effect. In the acquired form I have always found the iodine preparations the best remedies; local applications of the tincture or ointment of iodine at intervals of two to three days, subcutaneous injection of a solution of iodine, together with the internal administration of iodide of potassium ınd iodine waters, as those of Halle, Kreuznach, Krankenheil, Adelheidquelle, \&c., are to be recommended, though all are to ve used with caution.

If the afflicted children belong to a goitrous district they must be removed for a long time from its influences.

Cystic bronchocele has been treated successfully (Bruns and Demme) by splitting the cyst and fixing the edges of the wound to the walls of the cyst.

-

\section{e.-Diseases of the Lungs and Bronchi}

\section{Bronchial Catarrh, Acute and Chronic; Dry Bronchial Catarrh}

Bronchial catarrh, next to intestinal catarrh, is the most common disease of infancy. At the Prague Hospital for Sick Children, out of an annual clientèle of 9000 patients, there are on an average 1300 children suffering from catarrh of the airpassages. 
It may be either primary or secondary, acute or chronic.

Anatomy.-The bronchial mucous membrane is injected either generally or in patches, swollen, with velvety patches, and spread over with muco-purulent exudation, rich in cellgrowth. The bronchi become thickened and cylindrical when the affection has been of long duration, and: are sometimes sacularly dilated. These alterations are either confined to the trachea and its chief ramifications (tracheo-bronchitis), or they extend into the smallest bronchi (capillary bronchitis).

Atelectasis and emphysema of the lungs, and sometimes also inflammation of the pleura, may be results of catarrhal affections of the air-passages.

Symptoms and course.-According to the age of the child and the severity of the attack these may be slight and transient, or, on the other hand, they may be very serious and involving risk to life. Cough is the most important symptom, and at the onset of the attack is hard, dry, and painful, and in some cases, as previous to the outbreak of an attack of measles, is especially tormenting. In a few days it takes on a more rattling character.

Along with the cough difficulty of breathing occurs, which is always the more serious the younger the child is, and sometimes this is so marked that from fifty to seventy respirations in the minute may be counted, and all the supplementary muscles of respiration may be seen in activity. When sucklings are attacked they either refuse the breast or take it sparingly. Older children complain of pain in the breast and in the back. Palpation is of great use in this affection, as by it râles of various quality may be readily detected, especially over the dependent parts of the lungs. A râle, which is merely the result of a collection of mucus in the larynx, will disappear after a cough. Percussion does not yield much information, for beyond a more or less tympanitic note it gives no evidence of alteration. On auscultation may be heard rough, vesicular breathing, coarse or fine mucous râles more or less abundant, and the fine râles especially in capillary bronchitis. When the secretion is small in quantity the râles are snoring or shrill and piping. Sometimes the whole respiratory movement is occupied by extensive and loud râles. Sometimes the febrile indications are only slight, but at other times severe, especially in the cases of sucklings. If the catarrh be severe, especially if it affect the capillary bron- 
chioles extensively, the child gives indications of suffering and restlessnesss; the face is slightly swollen and cyanotic, the eyes staring, and an abundant perspiration breaks out on the forehead; the pulse becomes small, the skin cold, and the urine deficient in quantity, with a deposit of lithates. At the height of the disease symptoms of cerebral disturbance appear, due partly to congestive hyperæmia and partly to carbonic acid poisoning, and death rapidly ensues. Acute bronchial catarrh readily becomes chronic, especially in scrofulous and rachitic children. Under such circumstances the symptoms vary according to the time of examination, the cough being generally moist with an abundance of muco-purulent secretion, though sometimes, especially during the night, it is drier. Bronchiectasis, bronchopneumonia, and a certain form of broncho-phthisis are frequent complications and sequelæ of chronic pulmonary catarrhs. In some children an extremely obstinate and sometimes extensive bronchial catarrh occurs during the early weeks or months of life, and may last for years in a chronic form with occasional acute exacerbations. Such children have usually difficult breathing accompanied by loud râles, and also, especially at night time, sovere attacks of coughing, resembling those seen in hooping-cough and ending very often in an attack of vomiting, during which masses of mucus are evacuated.

I have met with a peculiar condition in children between one and four years of age, where the mucous membrane from the bifurcation to the bronchioles has been found unusually hyperæmic and much swollen, though without any secretion or with only slight traces of it. This may be called bronchitis sicca, and accompanying it I have found emphysema and hyperæmia of the lungs, destruction of the alveoli, hypertrophy and cheesy alteration of the bronchial glands, passive hyperæmia, and serous effusion in the brain, together with general wasting and anæmia.

Symptoms. - These consist of difficult and painful breathing, with occasional asthmatic attacks, and a frequently recurring irritating cough with occasional severe paroxysms, accompanied by perfectly dry and piping râles, and with a complete absence of expectoration. The percussion-note is seldom completely tympanitic, but more or less so, and anscultation reveals rough vesicular breathing and dry râles. Children thus affected gra- 
dually waste away, and die after a duration of the disease of from eight to eighteen months.

Causes.-These may be such as to excite the disease primarily, as exposure to cold, damp dwellings, inhalation of an atmosphere containing mechanical or chemical irritants ; or the disease may be secondary and symptomatic, occurring in the course of some other acute or chronic affection, such as measles, hoopingcough, rickets, scrofula, syphilis, pneumonia, croup, typhus, and, more rarely, variola and scarlatina. There seems to be a special disposition to catarrhal affections in some children, and even in some families, and in them the frequently occurring attacks of bronchial catarrh are very obstinate.

Prognosis.-This may always be favorable when the catarrh is primary and slight; but if it be chiefly affecting the capillary bronchioles the prognosis must be given cautiously, and with special reference to the age and condition of the child. Extensive bronchial catarrh in young sucklings is always very serious.

Treatment.-Slight catarrhs require little else than confinement to a well-ventilated room, in which the temperature is kept even, together with the administration of nutrient and demulcent drinks. For the relief of the abundant bronchial secretion a few drops of ipecacuanha wine with a little oxymel of squills, may be given with some compound decoction of marshmallow, or small doses of sal ammoniac in some demulcent. When the difficulty of breathing is great an emetic will be found useful. If indications of carbonic acid poison are already present, as a rule emetics either do not act at all or not sufficiently. For the relief of the irritating cough such remedies as opiates, aqua laurocerasi, aqua cerass. myrrhæ; the extracts of hyoscyamus, belladomna, or cannabis indica, or the benzoated tincture of opium, may be administered. When the catarrh is accompanied by feverish symptoms a slight derivation, either from the skin or from the intestinal canal, will often prove of service, and the rubbing the chest and back with some fatty material will often relieve the cough. If the secretion is very abundant and the difficulty of breathing great, narcotic remedies must be used with great caution and to a very limited extent, and stimulants must be given.

For chronic bronchial catarrh and the bronchitis sicca steam 
inhalations, either simple or medicatory; and the external use of stimulant and opiate embrocations are to be recommended. In the case of the catarrh being the result of some special dyscrasy, as scrofula, syphilis, rickets or tuberculosis, if such can be determined, the patient must be subjected to special and appropriate treatment.

In the cases where there is a catarrhal idiosyncrasy the only treatment must be a cautious and gradual hardening of the child by daily bathing, at first in tepid, afterwards in cold water, by the clothing being not too warm for the time of year, and by a not too great anxiety to protect the child from the air and weather.

\section{Hooping-cough; Tussis C'onvulsiva ; Pertussis}

Hooping-cough is an epidemic, contagious bronchial catarrh, accompanied by spasmodic attacks of coughing.

Of the many anatomical lesions found, those only belong really to the disease which indicate catarrh of the air-passages. Of the numerous others, all are mere resulting complications, such as lobular and lobar pneumonia, bronchiectasis, emphysema, chronic broncho-adenitis, tubercle of the glands and lungs, pleuritis, pericarditis, hydrocephalus, pulmonary and cerebral (Löschner) apoplexies.

Symptoms and course.-The disease may be divided into three more or less distinctly marked stages.

The first, or catarrhal stage (stadium prodromorum, s. invasionis), begins with ordinary catarrhal signs, as frequent sneezing, running from the nose, lachrymation and reddening of the eyes, tickling in the throat, and a dry, frequent, irritating cough, which comes on especially towards evening and during the night, frequently hoarseness, or even attacks of pseudocroup, slight : feverishness at night, slight delirium, and general restlessness and malaise of the child. This stage may last from a few days to a fortnight, and during its progress auscultation gives no other signs than those of bronchial catarrh.

During the second : stage (stadium convulsionum vel nervosum) the cough becomes intensified, and there occur more or less severe attacks of spasmodic coughing. Each attack consists 
of numerous, fitful, spasmodic expirations, after which comes a loud, crowing inspiration; and finally, after one or more of these attacks, between which there have been short pauses, there is an attack of romiting, in which tough masses of mucus are ejected. During this process the face is a bluish red, the eyes are starting out, and the veins of the head and neck much distended. In some children these attacks are preceded by some premonitory symptoms, as a tickling in the throat, a sense of pressure or burning under the sternum, or a certain amount of anxiety or acceleration of breathing. A single attack may last from thirty seconds to three minutes, seldom longer; and from twelve to sixty may occur within twenty-four hours. The severity of the attacks are in an inverse proportion to their frequency, and they are more numerous during the night than during the day. The attacks are easily induced by any emotion or excitement, as anger or wilfulness, laughing or crying, hasty eating or drinking, sudden change of position; and if several children affected by hooping-cough are together, they will all cough if one begins.

During this stage physical examination in uncomplicated cases reveals a diffuse bronchial catarrh, evidenced by fine and coarse râles. If auscultation be practised during the attack the vesicular murmur will be found very fceble or altogether absent, and the percussion note somewhat dull, though it will be found clearer the moment complete inspiration occurs. If any pulmonary complications exist the signs peculiar to them will be present.

During this second stage there is either an absence of feverish symptoms, or if they be present they are subject to nocturnal exacerbations. Its duration varies from three to eight weeks, seldom exceeding the latter period, and during its course the most frequent and most important of the complications of the disease occur.

The third stage, or stage of remission, is characterised by chronic catarrh and blenorrhagia, and during its course the cough gradually loses its convulsive character, and the attacks become less severe and of shorter duration. The peculiar crowing inspiration also disappears, or occurs only seldom; the romiting and ejection of the greenish-yellow muco-purulent masses occurs with less difficulty, and the condition of the 
patient becomes one of ordinary bronchial catarrh, and disappears entirely in from two to six weeks. In exceptional cases slight relapses occur.

Hooping-cough does not, unfortunately, always follow this straightforward course, but may have complications and sequelæ of various kinds, often extremely obstinate and involving great risk of life. These may be of a mechanical nature, or may affect nutrition, or may be of a composite character, the mechanical lesions occurring usually in the second stage of the disease, the others in the third.

The complications most to be dreaded are capillary bronchitis and broncho- or croupous pneumonia, and when such pulmonary affections have become established, especially the diffuse croupous pneumonia, the attacks of coughing become less frequent and weaker, or entirely disappear, and the breathing becomes more frequent and difficult.

The mechanical lesions consist of interstitial marginal emphysema of the lungs, usually transient and seldom leading to pneumothorax or general emphysema; also hæmorrhage from the nose, mouth, bronchi and lungs, from the ears accompanied by rupture of the tympanic membrane, and extravasation of blood under the conjunctiva and into the loose areolar tissue of the eyelids. In severe cases involuntary passage of urine and frees is sometimes met with and also the formation of hernix, prolapse of the rectum, and of the urethra in girls, hypertrophy of the thyroid and heart, and ulceration of the frænum of the tongue, the result of injury during the forced extrusion of the organ over the sharp incisor teeth during the paroxysms of coughing. In edentulous children this latter injury is not observed. The cerebral symptoms that are often seen in children attacked by hooping-cough, as great restlessness on the one hand, or lethargy, stupor or convulsions on the other, are the result partly of mechanical lesions, partly of altered nutrition of the brain. Sometimes these symptoms are very severe, and it may happen that the child will die suddenly during an eclamptic attack. As sequelæ of the disease we meet with gastric diseases and symptoms, as loss of appetite and diarrhoea, also broncho-adenitis, acute and chronic tuberculosis, anæmia, dropsy and marasmus.

Croupous laryngitis, pericarditis and pleurisy occur not un- 
frequently as complications of hooping-cough. An attack of measles may occur during the course of hooping-cough, and the diseases often follow one another. Children who have just passed through an attack of pertussis and who may have bronchitis are apt to have spasmodic attacks of coughing similar to those they have previously suffered from.

Causes.-Hooping-cough may be regarded as a neurotic contagious bronchial catarrh, the contagious element of which probably resides in the epithelial and purulent matter of the expectoration. Poulat states that he has found spores of low organisms in the expired air, but I have not discovered any such in the expectorated mucus. The epidemics generally occur in winter and spring, and children from two to seven years of age suffer most, sucklings being rarely attacked, though I have seen them suffer from the disease when only two or three weeks old. Hooping-cough generally makes only one attack, second occurrences of it being very exceptional. Its period of incubation is probably from three to seven days, and schools, churches, and common playgrounds afford the usual centres of infection.

Diagnosis.-The peculiar course of the disease with its characteristic attacks of coughing, the long-drawn-out loud inspiration, and the epidemic extent of the attack, enable the diagnosis to be made without difficulty. The presence of the ulcers in the frænum of the tongue is also of use, though they are not always met with, and they may occur in simple bronchitis and during teething. The spastic convulsive attacks of coughing in chronic broncho-adenitis and in simple bronchitis in young children differ from hooping-cough in the absence of remissions and in the sharply-marked character of the attacks, also in the non-cyclic course.

Prognosis.-Simple hooping-cough is not a fatal disease, though it may become so through its complications and sequelæ. This refers especially to sucklings and to rickety, scrofulous children, or those already suffering from pulmonary affections, for whom hooping-cough has an especial danger. In different epidemics the mortality may vary from 2 to 15 per cent., Löschner having found it in 700 cases.

Treatment.-As a prophylactic the best means is to remove the still unaffected children out of reach of the epidemic influence, especially those that are delicate. The dietetic treat- 
ment of the affected children must consist. of easily digested, nutritious food, administered frequently and in small quantity, and for young children milk diet is the most suitable.

During the disease the child must be held as far as possible from mental excitement, and any misbehaviour ought to be overlooked. If there is no feverishness and the weather good the child may be taken into the open air for a short time, even in winter, but especially in summer, and this may be increased as it is found the attacks of coughing become less severe and less frequent. Change of air often cuts short the disease, though it does not always have a beneficial influence, and even removal to the sea-side does not always lead to the wished-for improvement.

In some children I have seen the hooping-cough suddenly return, even after its entire absence for two or three weeks, when the patients have been brought back to the seat of the epidemic. We have no specific remedy for this disease, and of all those which have been recommended belladonna, in doses of the extract of one tenth, one sixth, or one half a grain, three or four times a day, either alone or in combination with quinine.or the oxide of zinc, seems to me to be of greatest service. The dose of belladonna is to be gradually increased until symptoms of intoxication appear, the child being carefully watched the while. When there is abundant mucous secretion and no vomiting, the occasional administration of an emetic will be useful. For the removal of the tough, gluey bronchial secretion ipecacuanha and the alkaline carbonates, in the form of soda water, will be found useful. Inhalations of steam, either alone or medicated by bromine, have been recommended. From the inhalation of chloroform, of the vapour of gas-liquor, the use of sulphate of aniline, the subcutaneous injection of morphia, or: the systematic employment of the cold-water cure, I have seen no satisfactory result. Adams has recommended the administration of half a grain of chloral hydrate two or three times a day.

When complications set in they must be treated according to their nature. Sometimes there is considerable risk from the hæmorrhages, which I have seen induced by each attack, and be occasionally very severe, and in these cases, when the patients have vomited all remedies, I have found iced milk very useful. 
In the third stage, when there is abundant secretion and a suspicion of tubercle, quinine, tannin, and cod-oil are to be administered.

\section{Pneumonia-Inflammation of the Lungs}

'This forms a very frequent and important affection amongst cliildren. Löschner has found that 10,181 cases occurred at the Prague Hospital in ten years.

Pulmonary inflammation occurring in children may be divided into two chief varieties, according to their anatomical changes, their clinical history and their causes, as the croupous lobar pneumonia, which is generally primary, and the catarrhal lobular, also known as broncho-pneumonia, which is, as a rule, secondary. This usual division, though it has some practical value to commend it, is not without objection, for lobular pneumonia may occur which is not catarrhal but croupous, whilst broncho-pneumonia may include large sections of pulmonary area, or even an entire lobe. Further, cases must have occurred to every experienced practitioner where it was difficult to discriminate between one form and the other.

\section{(a) Catarrhal Pneumonia-Broncho-Pneumonia- Lobular Pneumonia}

The anatomical changes in this disease consist, as the term lobular pneumonia indicates, in the formation of sharply defined bluish or dark-brown masses, generally depressed somewhat under the level of the unaffected lung-tissue, firm to the touch, and capable of distension through a bronchiole either imperfeetly or not at all. This condensation may be produced in various ways, most frequently by a catarrhal secretion, shed epithelium or abundant cell growth in the hyperæmic alveolar tissue, or by a molecule and cell growth external to the vesicles, or, what is least frequent, by a fibrinous exudation into the alveoli. The point of origin of these alterations is frequently atelectasis pulmonum. When a section is made through such a spot the cut surface is sometimes dry, sometimes slightly moist, or occupied by a frothy or bloody-coloured fluid; or the lung. 
tissue may be of a liver-like consistency, in the form of leaves, and with the cut surface fine or coarse-grained. I have seen these several varieties in the same specimen. In this condition the bronchial mucous membrane shows catarrhal alteration of various extent, often into the finest capillary bronchioles, with muco-purulent secretion and dilatation of the air-tubes.

As further results of this condensation there may be found softening and absorption of the increased elements, with freeing of the alveoli, hard cheesy exudation, or, more rarely, gangrene and the formation of abscesses. The chief seats of lobular pneumonia are the posterior and dependent parts of the lower lobes, rarely the upper lobes, and both lungs are more commonly affected than one only. The pleura may not be implicated, or it may have adhesions, either of delicate fibres, or of a more dense nature, corresponding to the affected parts of the lung. Hyperplasia, or cheesy alteration of the bronchial glands, is almost always present.

Symptoms and course.-The symptoms of the disease may be developed somewhat insidiously, or in a rapid and alarmingly severe manner. After indications of catarrh of the air-passages have existed for a longer or shorter period, symptoms such as restlessness and peevishness with some feverishness occur, the breathing becomes impeded and superficial, and the respiration increased in rapidity to as much sometimes as sixty to eighty in the minute. The expiration becomes sharply marked, the previously moist cough becomes dry, harassing and painful, the appetite is lost, and in sucklings the breast is either altogether refused or taken for short intervals. The voice is not altered, unless in very extensive disease, when it becomes hoarse and deficient in resonance, and the expectoration, when seen, which is not often, is found to be muco-purulent, or slightly bloody. In very young children there is often a frothy secretion between the lips. The face is at first somewhat reddened, but afterwards becomes pale, or even cyanotic. The temperature curve does not show the extreme exaltation seen in croupous pneumonia, nor does it indicate critical periods as in the latter disease.

Palpation discovers moist râles when there is extensive bronchial catarih, and percussion yields a tympanitic note, save when the lobular centres are large or confluent, when there is 
a somewhat dull note, but not to so marked an extent as in croupous pneumonia.

Auscultation reveals the vesicular murmur sometimes to be coarse and sharp, sometimes diminished in intensity, together with fine or coarse irregular râles, and towards the base firm râles are heard which seem close under the ear, with sometimes distinct crepitation, bronchial breathing and bronchophony, but only when the inflamed centres are large, and not then with the intensity and to the extent met with in croupous inflammation of the lungs. The course of the disease is completed in a short time, as is especially the case in the idiopathic broncho-pneumonia, or in that which is the result of measles; or it may become subacute, or even chronic, and may then last weeks or even months, the patient alternately improving and retrograding until some marked crisis occurs, or until some such result as bronchiectasis, contraction, or phthisis of the lung has occurred.

In small weakly or rickety children the disease usually ends, after a short course, in death, with symptoms of asphyxia.

Complications affecting the digestive and nervous systems are often met with, as diarrhœa with slimy stools, convulsions, somnolence, and, in the case of older children, delirium.

Causes.-This disease occurs most frequently between the ages of six months and three years, and is oftener met with than the croupous form of pneumonia. Catarrh of the air-passages seems the chief exciting cause, occurring either independently or in the epidemic form, or, what is very frequently the case, the catarrh which accompanies the course of measles, hoopingcough, rickets, scrofula, and croup. More rarely pneumonia is due to this cause during attacks of variola, scarlatina, and typhoid, and its occurrence is especially favoured by continuous lying on the back in the cases of weakly reduced children. As indirect causes of pneumonia may be mentioned rapid alterations of temperature, imprudent behaviour in the bath, injudicious attempts to harden the child and insufficient clothing.

Diagnosis.-This may be a matter of ease or one of considerable difficulty, according to circumstances, especially when it becomes the question to decide, between the catarrhal and croupous forms of pneumonia. The development of the disease from a primary or symptomatic catarrh of the air-passages, the protracted course marked by an uncertain irregular temperature 
curve, the absence of decided critical days, the ambiguous onset and the protracted remission, when taken in connection with the signs before mentioned, may all generally be taken to indicate the presence of catarrhal pneumonia, and the cases are only exceptional, where under such circumstances there can be doubt about the diagnosis. It is not always possible, even by the most careful consideration of all the symptoms, to distinguish between catarrhal pneumonia and acute pulmonary consumption.

Prognosis.-According to all experience this is always uncertain, and is the more unfavourable the younger the child is and the more reduced its condition of nutrition. According to $\mathrm{my}$ own experience two-thirds of the affected children die, and I may mention that the statistics of the hospital practice give more unfavourable results than those of private practice. Vogel lost half of his cases; Valleix 127 out of 128; and Ziemssen 36 out of 98 . The duration of the disease may vary from a fortnight to several months.

When the respiration becomes very much accelerated or very much retarded and irregular, and when there occurs at the same time sudden remission of the intensive cough which had previously characterized the case, together with a blueness of the hands and feet, depression of the temperature and convulsions, they may be regarded as the unmistakeable indications of an approaching fatal issue.

Treatment.- The fact that catarrhal pneumonia is usually found to proceed from a bronchial catarrh must lead us to be especially careful in all cases of catarrh occurring in sucklings or in children up to three years of age, and any measures are to be employed which will tend to relieve the catarrh. When there is profuse bronchial secretion, ipecacuanha, oxymel of squills, and antimony may be administered, and emetics will be found useful when the respiration is difficult and suffocation is threatened. When the cough is very troublesome narcotics may be given, but their administration must be undertaken with great caution. These may be such as the benzonated tincture of opium in doses of two to four drops, the syrup of diacodeia, Dover's powder in half or third of a grain doses, the extract of cannabis indicus in doses of a sixth to half a grain, or the extracts of hyoscyamus, belladonna or lactucarium. Stimulant 
remedics will, however, be found far better, such as the ethereal tincture of the acetate of iron, the liquor ammoniæ anisata, benzoin, and wine; and these I am in the habit of using, not only when there are symptoms of threatening collapse, but in the earlier stages of the disease. Care must be taken also to avoid all depressing influences, and to support the strength by appropriate tonics and nourishment. Cold applications to the chest have been recommended by many, but they must be employed with caution, for I have frequently seen their use followed by an alteration for the worse in the condition of the patient. Quinine is useful as a febrifuge, and a frequent change in the position of the child will often afford relief.

\section{(b) Croupous Pneumonia}

Anatomically considered, the acute lobar or croupous pneumonia in children exhibits the following alterations in the pulmonary tissue similar to those found in adults.

In the first stage of the disease-that of inflammatory congestion-the lung-tissue is darkish-red and swollen, firm and containing a diminished quantity of air. In the second stage - that of red hepatization-a fibrinous extidation is deposited in the alveoli, the bronchioles are gradually emptied of air, and the surrounding tissue becomes thickened, firm and of a brownishred colour, and presents a regular and finely.granulated surface on section. The third stage is that of grey hepatization, in which the dark-red colour of the lung-tissue passes into a yellow and finally to a yellowish-grey, from the increase of the exudation and the exuberant cell-formation. By incomplete celldevelopment the new exudation becomes broken down into a cream-like fluid, by which the lung-tissue is infiltrated, constituting the stage of purulent infiltration.

If a large part of a lung is affected the impression of the ribs is often seen on the voluminous lung in the stage of hepatization.

Both in the stages of red and yellow hepatization the alveolar exudation may be removed by absorption or by expectoration, and the pulmonary tissue be restored to its normal condition. Should neither this nor the occurrence of resolution take place, 
the further progress of the disease results in cheesy deposit, suppuration, more rarely gangrene, and, very exceptionally, induration or cirrhosis of the lung-tissue.

As a rule, two or more of the stages are found existing together.

Besides these alterations in the lung there may be present such conditions as bronchial catarrh, hyperæmia, or œdema of the part of the organ not inflamed, pleuritis, pericarditis, passive hyperæmia of the brain, liver, and kidneys, and occasionally meningitis or nephritis. These may be either primary or secondary with reference to the pneumonia.

Croupous pneumonia occurs more frequently on the right side than on the left, and in the lower lobes of the lungs than in the upper, though pneumonia at the apex on the right side is not unfrequently met with in children.

Symptoms and course.-In the primary form the disease is apt to occur with but slight warning, though in many cases, especially in more advanced children, it is ushered in by a wellmarked rigor or at least a feeling of chilliness, with vomiting and occasionally by an eclamptic attack. These initial symptoms are soon followed by fever and disturbance of the respiration, the child becomes restless, and its sleep is disturbed by horrid dreams, from which it awakes crying. The temperature rises to $40^{\circ}$ or $41^{\circ}$, and the pulse to $140-160$ in the minute, and is felt full and bounding. The breathing is markedly accelerated and very superficial, the inspirations being short, interrupted, and painful, and the expirations distinctly audible and accompanied by moans. The nasal cartilages display active movements, and the cry of the child is subdued and whimpering.

The cough is generally dry, frequent, and painful, though when there is bronchitis also present it is moist, and in very young children it may be altogether absent. Expectoration seldom occurs in children under six years of age, and when it is seen it is generally more or less mixed with blood, and in the later stages becomes rust-coloured.

The physical symptomsare very important and distinctive. In the first stage they are as follows:-Mensuration gives the normal girths, palpation sometimes discovers increased vocal fremitus, percussion yields a distinctly tympanitic note, or one 
somewhat shorter and slightly dull, and on auscultation fine mucous râles, crepitation, and an ill-defined, weakened vesicular murmur are heard.

During the stage of hepatization (red and yellow) these signs are so altered that mensuration shows a distinct increase in the circumference of the chest, especially of the affected side, and the note elicited on percussion is dull, the degree and extent of this corresponding to the extent of the consolidation and its proximity to the chest-wall. Hepatization, which is central, extending from the hilus of the lung and only gradually reaching the surface, does not produce the dulness on percussion for some days. If there be small districts of normal lung-tissue situated between others where there is condensation the crackedpot sound will be found. On auscultation, loud, distinct bronchial breathing is heard, as if very close to the ear, and there may be also tinkling râles and bronchophony.

During the stage of resolution the rocal fremitus resumes its normal degree, the dull percussion-note passes more or less rapidly into the tympanitic or moderately clear tone, and finally resumes its perfect resonance; the bronchial breathing and bronchophony disappear, fine râles take their place mixed more or less with coarser sounds, and afterwards the coarse râles usurp a prominence until the normal vesicular murmur becomes fully established.

As a rare condition, I may mention what $I$ have seen in several cases where the stage of resolution was followed by a process of complete absorption without the occurrence of any moist râles, the bronchial passing into vesicular breathing in a period of from two to five days, without any appearance of râles.

The ratio of the temperature is very characteristic in croupous pneumonia, rising, as it does at the very onset of the disease, and reaching during the first day as high, perhaps, as $40^{\circ}$ or $41^{\circ}$, at which elevation it remains with but slight variations until the occurrence of resolution.

At the end of three, five, or seven days, or, perhaps, even a longer period, the temperature begins to fall, and coincidentally, when the crisis is a complete one, there is a diminution in the frequency of the pulse and respiration. This typical depression of the temperature is varied in those cases where the inflamma- 
tion extends to fresh areas, either when the earlier hepatization is still in existence or where it has passed into resolution-cases which may be known as intermitting, remitting, or progressive pneumonia. The pulse frequence generally keeps pace with the temperature, and may rise to $130-160$, in very young children even higher, and only in very exceptional cases remaining low while the temperature is high.

Occasionally between the second and fifth day an eruption of congestive erythema may occur, especially on the trunk, and sometimes to a considerable extent, which, in connection with the high temperature, may be mistaken for an attack of scarlet fever. Its true character is readily determined by its transience and the absence of desquamation. The occurrence of herpes facialis is common in children suffering from pneumonia, its general seat being the lips, more rarely on the forehead, cheeks, or ears. The urine is diminished in quantity, and contains an increased quantity of uric acid and urates, a deficient amount of chlorides, and occasionally albumen.

One form of croupous pneumonia, differing in its course from the usual character of the disease in its severe cerebral symptoms, and peculiar to children, has been described by Rilliet and Barthez as cerebral pneumonia. It possesses more the character of a meningitis than a pneumonia, and the abovenamed authors have defined two varieties of it-the eclamptic and the meningeal-according to the predominance of convulsions or delirium. Pneumonia of the apices or of the central region of the lung in children frequentiy shows this variety. The causes of these cerebral symptoms are not always the same, and sometimes several have a coincident influence. Amongst these are to be mentioned the general tendency of the brains of children to sympathise with the action of inflammatory processes in other organs, the cerebral hyperæmia induced by increase of temperature, the passive hyperæmia of the brain and its membranes, the coincidence of suppurative otitis with the pneumonia, a toxæmic influence on the nervous system, or, finally; the brain symptoms may be reflex or due to a complication of meningitis.

Disturbances of vision or transient blindness, as sometimes occur in croupous pneumonia, are due to cerebral anæmia or to a similar condition of the retina (Gräfe and Henoch). 
A complete crisis, as a prelude to recovery, is marked by a diminution of the temperature and of the frequency of the pulse and respirations, and by the skin becoming moist, or even freely perspiring. The cough becomes more frequent, freer, and painless, sleep and appetite return, and diuresis occurs.

Pleuritis occurs as a complication of croupous pneumonia, and may have the position either of a precedent, coincident, or sequent affection, the first of these conditions being known as pleuro-pneumonia. The symptoms of this complication consist of severe stitches in the side, and short, superficial breathing, with a dry, obstinate and painful cough (and, as a physical sign, the occurrence of friction-sounds must be mentioned). Other complications may also be met with, as intestinal catarrh, pericarditis, bronchitis, meningitis, suppurative internal otitis, and slight jaundice which I have seen several times in sucklings as the result of passive hyperæmia of the liver.

The course of the disease is very characteristic, and its duration varies from five to ten days, being generally shorter in children than in adults.

The most frequent result is recovery, and this may take place, I am convinced, even in the stage of inflammatory congestion. The occurrence of cheesy alteration of the exudation, gangrene, or the formation of abscess, is not frequent in children. Absence of any indications of resolution and the prolonged continuance of the fever, together with the expectoration of putrid purulent matter, are to be regarded as serious symptoms.

In very extensive and severe pneumonia death is the result of suffocation produced by œdema of the remaining lung-tissue not inflamed. It may also result from cerebral œdema or other of the above-mentioned complications.

Causes.-Croupous pneumonia may be congenital. When acquired, that is when not congenital, it is generally a primary affection, seldom secondary, and sometimes it is quite epidemic in the extent of its occurrence, though generally the cases are sporadic. It may be due to climatic variations, to breathing an impure, noxious atmosphere, or to residence in cold damp dwellings.

Boys are more frequently attacked than girls, for of 1000 cases I have found 610 of the former and 390 of the latter. An attack of pueumonia frequently leaves behind it a tendency to 
recurrence of inflammatory attacks, for I have known it recur three, four, and even five times, before the completion of the dental change. It may occur in strong and healthy children as well as in those who are delicate and weakly. As a secondary affection it occurs in the course of such diseases as croup, the acute exanthemata, typhus, rickets, pyæmia, rheumatism, diseases of the brain, intermittent fever, and the sclerema of the newborn, and also as the result of metastatic embolism in the pulmonary arteries.

Diagnosis.-This may sometimes be a matter of difficulty, as the physical examination of children is not always easy, whilst the expectoration and the subjective symptoms may be altogether absent, or be only such as can be guessed at. Pleurisy differs from pneumonia in the greater severity of the pain, the short harassing cough, comparative immobility of the affected side during respiration, diminished vocal fremitus, increased sense of resistance or percussion, depressed position of the diaphragm, displacement of the heart and the friction sound, the latter sign being of unfrequent occurrence in children. To distinguish between cerebral pneumonia and meningitis during the first days of the disease may not always be easy; but the high temperature, the typical course, and, above all, the physical symptoms peculiar to it, will soon discover the pneumonia. Atelectasis may be distinguished from pneumonia by its feverless course, and by the diminished vocal fremitus.

Prognosis. - This is, as a rule, more favourable in children than in adults. The prognosis becomes more favourable the more advanced the child is and the better its health; also in proportion to the limit of the inflammation, the regularity of its course, and the unimportance of its complications. Primary pneumonia gives better ground for a favourable prognosis than when the lesion is secondary. The ratio of mortality is differently given by various authors from four to twenty-five per cent., and, according to my own experience, it is between five ąnd eight per cent.

Treatment.-This must be chiefly dietetic and symptomatic. Absolute rest, careful regulation of the temperature of the sick room at about $69^{\circ}$ Fahr., together with frequently administered drinks of lemonade or demulcent infusions, are almost sufficient for mild cases. During the stage of inflammatory congestion, 
stimulant applications to the skin, as sinapisms or turpentine stupes, are of undoubted service. Abstraction of blood had always better be avoided in children. When the fever abates, quinine, digitalis, nitrate and acetate of potash, and the tincture of veratrum may be given; but it must be noticed that digitalis and veratrum are apt to disturb the digestion and produce toxic effects. For the relief of the troublesome cough some calmative may be used with benefit, as the aq. laurocerasi, Dover's powder, or the extracts of hyoscyamus or belladonna ; and during the stage of resolution expectorants, especially ipecacuanha, in combination with a few drops of the liquor ammoniæ anisata, are the best remedies. To weakly and reduced children with extensive hepatization and a tendency to collapse, stimulants must be freely administered, such as wine, the xtherial tincture of the acetate of iron, \&c., and these I have often seen have a wonderful effect, and indeed to them I trust chiefly in the treatment of inflammatory diseases of the lung in children. Should the pneumonia threaten to end in induration or cheesy alteration of the lung tissue, the child must be removed to a mild climate, and especial care taken to avoid catarrh and to treat it at once should an attack occur. Cod-liver oil in such cases is invaluable.

The occurrence of abscess or gangrene demands treatment appropriate to each case.

\section{Pulmonary Emphysema*}

This is a pathological condition in which the air vesicles are enlarged by the loss of the elasticity and contractility of their walls, or, by the atrophy and destruction of the latter, the vesicles coalesce into larger cavities; and it is much more rarely seen in children than in adults as a substantive affection, though, on the contrary, a transient and slight degree of it is not unfrequently found in children.

Anatomically there is a distinction made between vesicular and intcrlobular emphysema. In the former the alveoli are distended, appearing on the surface either isolated or in groups, occupying spaces from the size of a pea to that of a hazel-nut, showing on the surface distended vesicles with much-thinned 
walls. The tissue is anæmic, seldom marked with pigment, and the vessels are atrophied and their walls destroyed. The seat of the emphysema is generally at the apices and anterior edges of the lungs.

Interlobular emphysema, or the extravasation of air into the interstitial cellular tissue, occurs from the rupture of the air vesicles. It appears on the surface as small bladders or streaks of air under the pulmonary pleura. In some cases, this membrane being torn, the air escapes into its cavity, whilst in others the air passes into the tissue surrounding the bronchi, and in others into the anterior mediastinum, thence passing into the cellular tissue of the neck and under the skin (emphysema cutaneum).

The immediate causes of emphysema are repeated forcible respirations, and probably simultaneous disturbance of the nutrition of the alveolar walls, and for its production such conditions as catarrhal and dry bronchitis, croupous pneumonia, croup, hooping-cough, tuberculosis, struma, and foreign bodies in the air-passages are especially favourable. Compression of a part or of the whole of a lung may also predispose to the occurrence of emphysema in the tissue which still remains patent, as in extreme curvature of the vertebral column, or in displacement of the bones of the chest, and it also may be produced during the agony. Whether or not it is sometimes produced by the insufflation of still-born children must be regarded as doubtful. I have noticed that in some families emphysema seems to be hereditary, due no doubt to the inherited tendency to catarrhal affections. Hecker and others have noticed it as occurring congenitally.

Symptoms and course.-When the extent of the emphysema is limited it does not afford any clinical indications of its existence; but when it is diffuse and of long duration, it gives rise to the following train of symptoms: the capacity of the chest is increased, though it does not present the peculiar barrel-shaped enlargement which is the result of emphysema in adults; the diaphragm is depressed; the breathing is laboured; the peripneumonial furrow is present; the cardiac dulness is diminished, and the apex beat weakened; the pulse is small; the cutaneous veins distended and enlarged; the face somewhat swollen; and the skin pale or cyanotic. Percussion yields a full, 
clear, and tympanitic note, and on auscultation the vesicular murmur is found to be weak; and if there be any catarrh of the bronchi present there will be piping or snoring râles. These signs may, however, be marked more or less by the symptoms of the primary disease.

As this disease is seldom permanent in children, its results and complications, such as passive hyperæmia of the liver, spleen and kidneys, and hypertrophy of the heart, are seldom present, or so only to a very limited extent. Sometimes, however, a passive hyperæmia of the brain may occur as a result of emphysema, and become a source of danger to life.

Resolution of the emphysema is the rule in children, and this occurs rapidly, as we see in hooping-cough, where it is almost always present. It is seldom a cause of death, that, when it occurs, being due to the primary disease.

Prognosis.-This is always more favourable in children than in adults, though in considering it regard must always be had to the conditions of the disease, its complications and extent, and to the age of the child.

Treatment.-The emphysema can be affected only in an indirect way and to a limited extent by treatment, which must be directed against the primary disease, or against any complications that may arise. Thus, should it arise in the course of hooping-cough or bronchial catarrh, the treatment and precautions appropriate in these affections must be carried out, and in the case of struma iodine must be given. To relieve the diffculty of breathing, especially in debilitated children, stimulants such as wine, liquor ammoniæ anisata, and benzoin form the most suitable remedies, in conjunction with nutritious diet and the application of irritants to the skin of the chest. The inhalation of vapour, either simple, or medicated by the addition of sal ammoniac, turpentine, \&c., has been recommended. 'The use of compressed air and electricity ${ }^{\bullet}$ do not seem to have had the successful issue which was anticipated, and the remedies most generally now in use are nux vomica and strychnia. Removal to a warm climate during winter and to the influence of sea-air, is to be recommended when practicable. 


\section{Atelectasis pulmonum}

Atelectasis is any non-inflammatory condition of the lung by which either the whole or sharply defined districts of it remain undistended by air, or become so, and, as a result, the alveoli collapse. It may be congenital, in which case it is a continuance, to a certain extent, of the fotal condition of the lung, or it may be acquired.

Anatomy.-The atelectasis may be found to affect considerable sections of the lung, or it may be confined to minute centres; and it is met with chiefly at the posterior and inferior parts of the lower lobes and along the free edges of the lungs, in the form of spots, depressed under the level of the nominally distended lung tissue and altered in colour to dark reddishbrown or blue, or even to a steel-blue tint. These spots are lax and soft to the touch, sink readily in water, may be distended by blowing in air, and when cut into show a uniform and not granular surface from which exudes a fluid somewhat bloody and free from air-bubbles. When the pulmonary collapse has been in existence for some time these spots are found to have a somewhat firmer consistence, resembling that of flesh, or even harder. In the bronchi of atelectic lungs hair and meconium have been found.

Symptoms and course.-The symptoms of atelectasis consist chiefly of those of obstruction to the circulation and respiration. Children with congenital atelectasis are frequently still-born; and when breathing does begin it is laboured and insufficient, the efforts at insfiration being accelerated, superficial and irregular, and the hollow between the chest and abdomen is exaggerated (the peri-pneumonial furrow). The child does not cry loudly, but gives vent to weakly whimpering tones, lies quietly at the breast, sucks languidly or not at all, and sleeps a great deal. The face is more or less cyanotic and wan, the temperature is low, and the pulse small. If the severity of these symptoms should increase and convulsions occur, death will take place a few days after birth, unless the respiration becomes more fully established. In the latter case the indications of insufficient respiration and oxidation of the blood dis- 
appear, so that in a few weeks or months the condition of the affected lung becomes normal.

The symptoms both of acquired and congenital atelectasis, whèther in children or in adults, exhibit various degrees of severity in proportion to the extent of the affection, and its more or less acute development. When there are no complications symptoins of fever are almost always absent.

In slight cases physical examination discovers hardly any appreciable signs, though when the emphysema is extreme there may be decided indications of its presence. In cases of new-born children mensuration is of little use, but in those a few weeks or months old the entire circumference will be found under the normal size when the emphysema is double; and when one side only is affected, the corresponding side of the chest will give a less measurement than the other. Only when the emphysema is extensive and superficial will percussion yield a somewhat dull note, and in auscultation will the vesicular murmur be found weak. In less marked cases these two methods of examination will give only negative results. In new-born children with atelectasis pulmonum the respiratory sound is often hardly audible, and in other cases, when there is also catarrh present, it is marked by moist râles. Occlusion of the larger bronchi is indicated by a weakening of the rocal fremitus, which is again restored when they become patent. Hæmorrhage into, and œdema of, the unaffected lung tissue, small frequent pulse, intensified heart-beat and increase of the cardiac dulness, cyanosis and œdema of the skin, passive hyperæmia and œdema of the brain, thrombosis of the cerebral sinuses, albuminuria and persistent patency of the foramen ovale are all met with more or less frequently, as the result of the influence of atelectasis pulmonum on the circulation.

Causes.-These may be very various. Congenital debility, asphyxia and collection of mucous or amniotic fluid in the bronchi and bronchioles, may in certain cases be the causes of congenital atelectasis. In the acquired form the cause may be referred to three groups-occlusion of the bronchi, debility of the respiratory muscles, and compression of the lungs. To one or other of these groups may be referred such causes as bronchial catarrh, especially of the chronic form, impaction of foreign bodies in the air-passages, new formations in the lungs, 
exudations and collections of air or tumours in the pleural cavity, hypertrophy of the heart, dropsy of the pericardium; also exhaustive diseases, such as typhoid, cholera, chronic intestinal catarrh, long-continued dorsal decubitus, deformities and rickets of the bones of the chest, and elevation of the diaphragm from collections of gas or fluid in the abdomen.

Prognosis.-This will depend upon the extent of the mischief, the nature of its cause and the'possibility of removing the latter. Extensive atelectasis in a new-born child is always a serious condition.

Treatment.-This must always in great mcasure be governed by the cause of the disease. In congenital atelectasis the indications for treatment are first of all to clear the air-passages, if possible, of any obstructions that may exist, to sustain the strength of the child and to aid the respiratory process. The first may be accomplished to some extent by the administration of an emetic of squill and ipecacuanha, and the other by the administration of stimulants, as wine, the liquor ammonix anisata, and the æthereal tincture of iron; also by the application of irritants, such as sinapisms, \&c., to the skin, and by rubbing and brushing it; by the alternate immersion of the child in warm and cold water, and probably also by the use of electricity in various ways. If chronic bronchial catarrh be the origin of the atelectasis, the most beneficial treatment will be found to consist of expectorants and such inhalations as tend to loosen the mucous secretion. When it is accompanied by rickets cod liver oil and iron are to be given, and debilitated children must have stimulating diet, quinine and other tonics. All remedies which are likely to interfere with the action of the lungs, as narcotics, are to be strenuously avoided, even when troublesome attacks of coughing would seem to demand them.

\section{Phthisis pulmonalis-Consumption}

The term Phthisis does not define any independent disease of the lungs, but only the result of certain pathological processes, and has therefore no very accurate clinical value. Pulmonary phthisis may originate in tuberculosis, in lobar or 
lobular pneumonia, or in chronic bronchitis; and we establish thereby the varieties of phthisis known as tubercular, pneumonic, and bronchitic. The relative frequency of these varieties in children, according to my own experience, may be seen from the fact that out of fifty-two cases accurately investigated sixteen were found to have originated purely from pneumonia and bronchitis; eighteen were due to tubercle, and the remaining eighteen were of a mixed form, that is to say, chronic pneumonia and tubercle were coexistent, and it was impossible to decide which was the primary and which the secondary affection.

\section{Tubercular Phthisis}

Tubercle occurs in the lungs, either in the miliary form or in larger nodules known as the yellow and grey tubercle, and is due to an increase in cell-growth, either in the external walls of the capillary vessels, of the lymphatics, or in the interstitial cellular tissue; but whether this increase is inflammatory in its origin or neoplastic is not yet decided. The further changes found in the lungs of children are the formation of cavities and the destruction of tissue, and; very rarely, calcification; and these are found more frequently in both lungs than in one only, though, in the latter case, the right side is the more frequently affected, and the upper lobes are oftcner the seat of disease than the lower. Tubercle of other organs, as serous membranes, the parenchymatous organs of the abdomen, the intestines, and the larynx, is frequently associated with its presence in the lungs. It is a rule, almost without exception, that along with miliary tubercle in the lungs there will be found some old cheesy or purulent deposit, most frequently in the bronchial glands, sometimes in the lungs, or even in some distant organ, due, probably, to scrofula. It is very exceptional to find that the disease has no such focus of origin and is what may be termed idiopathic. Other pathological changes may be found which are not of a tubercular nature, as pneumonia, œdema and emphysema of the lungs, bronchial catarrh, pleuritic adhesions, fatty degeneration of the liver, sago spleen, fatty and amyloid degeneration of the kidney, \&c. 
Symptoms and course.-The disease may occur in a very acute form with violent symptoms, or more insidiously with a chronic character, being ushered in by symptoms apparently slight, and may even have a latent stage of varying duration.

The more important symptoms consist of frequently recurring catarrh with a dry cough which harasses the patient night and day, a feverish temperature with varying curve and occasional complete remissions, a gradual but very decided and progressive emaciation with blanching and withering of the skin, and a marked acceleration of the respiration, to as much as 50 or 60 or even 80 in the minute. The hands and feet are often very hot, the sleep is unquiet and disturbed by horrid dreams, the stools are generally normal, or become diarrhœic when there is tubercular alteration in the intestines. General hyperæsthesia of the skin is frequently noticed.

If the course of the disease becomes chronic the strength becomes gradually exhausted, or this may be the case even when the disease does not actively progress. The emaciation advances till the patient is reduced almost to a skeleton with his skin hanging loosely in folds; the cough becomes very troublesome, and may or may not be accompanied by expectoration. The further symptoms met with are delirium, dorsal decubitus, and œdema of or hæmorrhage into the superficial tissues. The course of the disease may be much modified by such complications as meningitis, peritonitis, \&c.

Physical examination does not always give decided indications Sometimes there exist only evidences of a severe catarrh or capillary bronchitis; and only in the presence of the large tubercles, of broncho-pneumonia or of cavities do we get such decided symptoms as dulness on percussion or cavernous breathing.

Death is the general result, and may be due immediately to secondary pneumonia, meningitis, peritonitis, \&c., or sometimes to the extreme degree of exhaustion.

Causes.-These have been already referred to in the general consideration of tubercle.

Diagnosis.-As has before been mentioned, the diagnosis of the miliary form is surrounded with difficulty from the entirely negative or only slight indications yielded by the physical examination of the thoracic organs; whilst in the coarser deposit 
this is not the case, from the greater size of the centres and the formation of cavities. It mat generally be possible to avoid mistaking miliary tuberculosis for typhoid fever by noticing the absence of the typical course of the pyrexial symptoms, the absence of the peculiar rash and of any enlargement of the spleen.

Prognosis.-Miliary deposit in the lungs is generally fatal, though there is a possibility of cure by induration and calcareous alteration of the tubercle. General tuberculosis of several organs is almost always fatal.

Treatment.-This is identical in part with the treatment of tuberculosis in general, and partly with that of pneumo-phthisis, which will be mentioned afterwards.

\section{Phthisis from Chronic Pneumonia and Bronchitis}

Chronic pneumonia may develop into pulmonary phthisis, and this may be its result either in the cases where it occurs in limited centres (chronic, disseminated, and catarrhal pneumonia), or in those where it is diffuse.

The anatomical alterations, which are always of an inflammatory origin, consist of dry nodules or isolated patches of a yellowish or even bright sulphury colour, sometimes scattered sparsely and sometimes very numerous, occurring generally in both lungs, and surrounded by miliary tubercles of subsequent date. If the phthisis be the result of a diffuse croupous pneumonia which has not advanced to the stage of resolution, the affected centres are of large size and may even occupy an entire lobe or the greater part of one, in that case the upper lobes being generally those affected. In some cases the chronic pneumonia does not advance beyond this stage, but there arises, in the yellow spots, a softening and disintegration of the mass by the destruction of the cells and by the spread of the inflammatory and ulcerative processes there are formed cavities of varying size. By the coalescence of several of these of small size, large, irregularly shaped vomicæ are formed, with ragged walls. Along with these are often found such appearances as bronchiectasis, brawny condensation and cicatricial retraction of the lung-tissue. If the cavities advance towards the pleura 
and perforate it, an occurrence which is not common in childhood, pneumothorax may occul. In such cases the pleura almost always contracts dense adhesions and becomes much thickened. Subsequent retraction of the chest-wall will be met with only in more advanced children.

Symptoms and course.-The signs of this form of pulmonary phthisis are essentially those of chronic pneumonia or bronchitis, and the peculiar physical and functional phenomena of those two diseases are characteristic of this affection at its outset. A pneumonic infiltration which has had a protracted course, and in which the stage of infiltration has either been only incompletely passed or not entered into at all, is the starting-point of this disease. Along with it there occur signs which point more or less clearly to the presence of cheesy deposit in lobular centres, these gradually becoming more abundant and being accompanied by obstinate catarrh, dry, troublesome and even convulsive cough. The development of the child does not keep pace with its age ; but, on the other hand, the weight diminishes, the child becomes melancholy, the skin blanched, dry, and covered with eruption; slight feverish symptoms with varying temperature curve make their appearance along with other suspicious symptoms, aud these may be set down as the symptoms of pulmonary phthisis, with all the more certainty if there be any scrofulous or tubercular taint hereditary in the family, or if in the child there be any evidence of previous glandular affection. If the phthisis advances with purulent destruction of the infiltration and of the lung-tissue, resulting in the formation of cavities, or if the formation of the cavities takes the form of bronchiectasis, along with the gradually progressing consumption there will be noticed a more or less abundant muco-purulent expectoration, sometimes greyish-yellow and nummular, sometimes bloody and containing the products of lung destruction. As a rule there is not much interference with or acceleration of the breathing from the presence of vomicæ, even when numerous and of large size, and when such alteration of the respiration is naticed it is usually the result of a fresh pneumonia. Hæmoptysis is not nearly so frequent a symptom as in adults, though $I$ have seen it in a child three years old. Such signs as cavernous or bronchial breathing, coarse mucous râles, and a dulled percussion-note occurring in such cases must always be cautiously interpreted, 
unless they are well marked and found to be persistent. General anæmia and obstructions to the circulation are often indicated by drcpsy, although that may also be due to amyloid degeneration of the kidney, and with these there may occur pyrexial symptoms which are at first uncertain, but which afterwards become more pronounced, being ushered in by a well-marked rigor, followed by alternating cold and hot stages. Hxmorrhagic petechiæ are sometimes seen on the skin in great numbers; sometimes there is an obstinate diarrhœa with mucopurulent stools, due to ulceration of the mucous coat of the intestine, or there may be hoarseness or even complete loss of voice from ulceration of the larynx.

Death may be either the direct result of the pulmonary disease, or it may follow from the onset of an attack of tubercular meningitis, peritonitis, or from a more general miliary infection. In the latter case the febrile symptoms become very much increased and remain so till death.

Causes.-As causes of this form of phthisis may be reckoned hereditary taints, such as scrofula, tubercle and syphilis, scrofula developed independently in the child, exposure to damp and contaminated atmospheres, residence in newly built and incompletely dried houses, or in a climate liable to sudden vicissitudes and storms, and also such diseases as hoopingcough and measles.

Prognosis.-Chronic pneumonia resulting in phthisis must always be regarded as a serious disease, though the possibility of its remaining in a passive condition, or even being completely recovered from, may always be entertained, the latter fortunate issue being specially likely to happen in cases where the formation of cavities has been the result of chronic bronchitis. The prognosis will be all the more hopeful when the outward circumstances of the patient are fortunate, when the digestion is good, and when the fever occurs seldom and is slightly marked.

Treatment.-This may be divided into that which is prophylactic, the dietetic, and that which is peculiar to the affection. Children born of a mother who either is or has been the subject of pulmonary disease must always, when possible, be provided with a nurse and be suckled by the mother as short a time as possible. Children should not be employed at all, or at least 
for very short periods, in manufactories where the atmosphere is rendered prejudicial by the presence of dust. In children near puberty in whom there is a suspicion of tubercle, or who may suffer from chronic pneumonia, care.should be taken to avoid exhaustion by too long hours, and such affections as catarrhs, measles, and hooping-cough are to be guarded against as far as possible by the avoidance of contagion. A most important part of the treatment consists in a non-irritating and nutritious diet, including such articles as milk, beef, and strong beef soup, eggs, acorn coffee, beer and diluted wine which has been matured, and such other articles of diet as are appropriate for the purpose. If the appetite is deficient then tonics are to be given, as quinine and the cinchona extracts, or other bitters, or small doses of Malaga wine. The treatment by drugs must be governed in great measure by the indications derived from the causes to which the disease can be traced. When this occurs in scrofula the most important remedies are the preparations of iodine, the iodide of iron, and, above all, cod-liver oil when it can be borne and when there is no diarrhœa. If the child is free from fever and very anæmic, some preparation of iron, alone or in combination with quinine, as the phosphate of iron syrup, or the iodised syrup of iron and quinine, will be found the most suitable remedy. When there is much irritation from the cough, relief may be obtained from opiates, given either alone or in combination with quinine, as the benzoated tincture of opium in doses of from four to six drops, as required; the extract of belladonna, in doses of a tenth to a quarter of a grain; or the extracts of hyoscyamus and cannabis indicus. I have seen very great relief from the following prescription :

Bo Quinæ Sulphatis, gr. ij ;

Pulv. fol. Digitalis, gr. vj;

Ex. Opii, gr. j;

Pulv. Sacchar. alb., ad $3 \mathbf{j}$ :

M. et divide in pulver. xij.

Sig.-One to be taken every three or four hours.

Pectoral tea mixed with milk may also be given. When there is very abundant expectoration, tannin and such balsams as tolu, and especially the oil of turpentine, are useful; and when the cough is severe and expectoration is difficult some stimulant, as the liquor ammoniæ anisata, may be given. When the fever is 
severe it may be diminished by large doses of quinine. The use of inhalations excites a good deal of opposition on the part of the children and does not seem, in my experience, to have had any good results. The pain in the chest, which is common in the course of this disease, may be relieved by the application of stimulant or opiate liniments to the skin of the chest. From the usually prolonged existence of the disease occasional variations in the treatment will be found necessary.

When hæmoptysis occurs hæmostatics, as ergot, alum, and the liquor ferri sesquichlor. will prove serviceable, and in severe cases the external application of cold packing or icebladders may be had recourse to. The complication of chronic pneumonia with tuberculosis of the lungs or other organs requires the same treatment as has been detailed as applicable to tuberculosis as a general disease.

\section{Gangrene of the Lungs}

Pulmonary gangrene is not often met with in children, though it has occurred forty times in my experience. It is characterised anatomically by a portion of lung, of greater or less extent and the limit of which may be sharply defined or be somewhat diffuse, in which the normal tissue is seen gradually passing into a condition of mortification, exhibiting a dark red, grey or green colour, rotten and easily torn, and either permeated by a badly coloured and offensively smelling fluid, or occupied by cavities of various size in which that fluid is collected. Microscopic examination of the gangrenous mass shows fragments of the alveoli and numerous crystals of inosteasin. The gangrenous spots occur more frequently in the deeper parts than in the periphery of the lungs, in the former case the pleura being thickened and discoloured, softened or even perforated, and in the latter case pneumothorax, with ichorous exudation, results.

Pulmonary gangrene in children is always a secondary process. The immediate cause is the compression or occlusion of the branches of the pulmonary artery supplying a certain section of lung tissue, producing an arrest of its nutrition, or in the staguation of some substance easily capable of 
decomposition, as for instance the fluid in cavities. These conditions may be the result of causes partly local-partly general. To the first variety belong lobular and lobar pneumonia, pulmonary phthisis, bronchitis, embolism of the pulmonary arteries, pulmonary apoplexy, and possibly marantic thrombosis and foreign bodies in the air-passages.

In the second class of causes which produce gangrene of the lungs, that is those which act through the general system, we may group those diseases which reduce the strength or alter the composition of the blood, such as typhoid, measles, smailpox, scarlet fever, diphtheria, chronic intestinal catarrh, chronic pneumonia, scrofula, malarious diseases, \&c. It occurs more frequently in boys than in girls, and may be met with as early as the third or fourth months of life.

Symptoms and course.-According as the causes have been in existence for a more or less protracted period, the signs of the pulmonary gangrene are more or less sharply marked. The child is apathetic, its look is weary and distressed, its strength evidently much diminished, the skin earthy and grey, and the pulse small and frequent. The most important sign consists of a disagreeable carrion odour of the exhaled breath, which does not arise from the mouth, nose or throat, but originates in the lungs. The sputa may be altogether absent, but when they are present they are muco-purulent, of a bad colour and most offensive smell, and streaked with more or less blood. The microscopic examination of the sputa shows puscorpuscles in a state of fatty degeneration, epithelium, drops of oil and crystals of the fatty acids, blood corpuscles, elastic fibres and débris of the alveoli.

'The physical examination of the lungs yields either only negative results, or, where well-pronounced morbid conditions exist, the signs peculiar to them. When the gangrene has led to perforation of the pleura the signs of pneumo-thorax will be present.

Recovery is a rare result; for out of forty cases which constitute my own experience, only two, in which a gangrene occurred in the course of typhoid, had such a fortunate result. Death occurs usually in two or three days, though occasionally it is delayed to the eighth or tenth.

Diagnosis.-As a rule there is little difficulty about this. 
The decisive signs are, the collapse of the patient, the peculiar earthy or grey colour of the skin, the gangrenous fotor of the breath and sputa, together with the microscopic appearances of the latter; and these, taken in connection with the history and the other physical signs and symptoms of disease, will exclude any other conclusion.

Treatment.-The most important indication is for the sustenance of the patient's strength. Free ventilation with pure air and the addition to the atmosphere of some disinfectant vapour for the destruction of the gangrenous ordour, and the exhibition of a strong but easily digested diet, consisting of soup, eggs, milk, beer and wine, are also necessary parts of the treatment. Among drugs the mineral acids, quinine, the extract of cinchona, and chlorate of potash are most in favour, my usual remedies being a combination of the latter two. Oil of turpentine, creasote, camphor and chlorine have been mentioncd as having a direct influence on the gangrenous centres, but the most potent seems to be oil of turpentine, administered either in the form of inhalation, or given internally in doses of ten or twelve drops in some mucilaginous emulsion.

\section{Pleuritis}

Pleuritis, as an idiopathic affection marked by sharply pronounced symptoms, is a much rarer disease in infancy than adult life. On the contrary, secondary and partial inflammatory affections of the pleura, not capable of diagnosis during life, are frequently found in children on post-mortem examination.

Anatomy.-External pleuritis is generally unilateral, rarely occurring simultaneously on both sides of the chest. It is usually found at the lower, postcrior, and lateral parts of the chest, but when limited it may occur as interlobular, mediastinal, and occasionally as diaphragmatic pleurisy. It may be parenchymatous or exudative, in the latter case the cxudation taking the form of flakes, membranes, or connective-tissue adhesions, or it may appear as free fluid in the cavity of the chest. These varieties, or some of them, may also be scen in existence together. The pseudo-membranes may adhere only loosely to 
the pleura, or their relations to it may be more intimate by the passage of vessels between them, and afterwards by the development of vascular processes they form permanent membranes of about a line in thickness.

The fluid effusion, which may vary in quantity from a few ounces to one or two pounds, consists of serum with some fibrinous flocculi, or it may be purulent and, in some exceptional cases, hæmorrhagic. When the effusion is very copious, and especially when it has been a considerable time in existence, the lung may be so compressed as to be deprived of air and blood, its function destroyed so that it is no longer capable of exhaustion, and the tissue be converted either wholly or in part into a substance like leather, whilst the unaffected lung becomes voluminous and affected by chronic catarrh, with dilatation of the bronchi. A pleuritic effusion may become capsulated by fibrinous adhesions, and then form what has been described as saccular exudation. As the result of extensive effusion, the cavity of the chest becomes increased, the intercostal spaces bulge, the diaphragm is pressed downwards, and, when the pleurisy occurs on the left side, the heart is displaced to the right. If absorption takes place in a short time these displacements are corrected and the organs return to their normal positions; but if it occurs only after the lapse of a considerable period the lung does not become fully expanded, the chestwalls sink in, and there is established the rétrécissement thoracique, with a compensatory scoliosis of the lumbar vertebræ.

Purulent effusion (empyema), whether free in the cavity or sacculated, may become absorbed or, after prolonged duration, may lead to perforation of the chest-walls or of the lung-substance, into a bronchus, or occasionally, as I have seen in the instance of a girl ten years old, into the œsophagus or into the abdominal cavity.

Limited pleuritis occurs along with lobar and lobular pneumonia, and generally leads only to the formation of pseudomembranes and adhesions.

Along with pleuritis we sometimes meet with peri- and endocarditis, pneumonia, meningitis, \&c.

Symptoms and course.-The disease may occur either in a very acute form with symptoms of severe fever, or it may have a 
more insidious and chronic course. The pulmonary symptoms consist of a well-marked rigor or slighter shivering, vomiting, and more or less severe stinging pain in the affected side; in sucklings there is great restlessness, distortion of the features, or even eclamptic attacks followed by stupor, and in more advanced children we frequently see delirium. 'These symptoms may, however, be absent and the nature and seat of the ailment be revealed only by the physical and functional alterations. In the further course there appears a short, dry and very painful cough, and the respirations become increased in frequency (from 40 to 70 in a minute), short, superficial, painful and laboured. The decubitus of the child is generally very still and on the affected side or on the back, seldom on the sound side. Children at the breast do not suck at all, or only do so for short intervals. The skin is blanched, yellowish, or even cyanotic when the effusion is great; and in the latter case, or when both pleuræ are affected, or when the effusion has taken place very rapidly, the cyanosis may become extremely marked, and the action of the accessory muscles of respiration become exaggerated. The febrile symptoms are usually marked with some degree of severity, the temperature varying from $40^{\circ}$ to $44^{\circ}$ Cent.; the pulse rises to 120 or 140 , and in very young children to as high as 180 . The temperature curve has no typical variations, though nightly exacerbations are often observed. When the exudation is purulent there is a continuous and extremely high temperature. The physical examination shows that the respirations are exaggerated and shallow, with deficient expansion of the affected side, distension of the intercostal spaces and diminished vocal fremitus. At first percussion yields a note only partially dull, but in the further course of the effusion it becomes completely dull with an increased sense of resistance on percussion. The vesicular murmur is either very much diminished in intensity, or disapappears entirely and is replaced by bronchial breathing which seems to be produced at a distance from the ear and has not the same loudness as that heard in croupous pneumonia. At the line where the exudation and the normal lung tissue border an exaggerated vesicular murmur is usually heard, or there may even be amphoric breathing. Friction sound is rarely heard in children, and is never met with in its complete form. When 
in addition to the pleurisy bronchitis or pneumonia is present, the signs and symptoms peculiar to them make their identification easy. Limited pleuritic effusion is not easy of diagnosis, and presents no perfectly trustworthy symptoms.

In chronic pleurisy the physical signs detailed above may last for months or even years, and in such cases the children seldom breathe freely, but suffer from dry cough, shortness of breath, palpitation of the heart and epistaxis. 'The colour of the face is waxy-white and transparent, and is only occasionally relieved by a transient flush. Any catarrhal affection of the lungs becomes in them very serious, and there are nightly exacerbations of the fever with marked night sweats. When the greater part of this exudation has become absorbed there results a marked deformity of the chest-walls, from retraction of the affected side, by which the ribs are drawn together and the spine becomes curved. In cases where the exudation per forates the wall of the chest, improvement follows the evacua. tion of the pus, though there usually remains a fistulous opening for a long time. If the perforation occurs into a bronchus the discharge is coughed up, or if into the osophagus it is evacuated by vomiting or at stool, as I have seen in one case.

When the course of pleurisy is acute, recovery with rapid absorption may take place within a week or a fortnight; when it becomes chronic, the recovery may be either complete or incomplete. When the exudation is of large amount, has been rapidly effused and is purulent, especially when there is superadded a pyæmic infection, it is very often fatal, though recovery may take place even after the disease has lasted for two or three years.

The most important complications of pleurisy are pneumonia, bronchitis, pericarditis, intestinal catarrh, tuberculosis and meningitis.

Causes.-The causes of idiopathic pleurisy cannot always be ascertained. In a secondary form the disease may occur after croupous and catarrhal pneumonia, hæmorrhagic infarctus, tuberculosis, pulmonary gangrene, disease of the heart, pyæmia, scarlet fever, smallpox, measles, Bright's disease, burns, congenital syphilis, abscess in the chest from caries of the vertebræ, suppuration of the right lobe of the liver with perforation 
of the diaphragm (Löschner), and hydatids of the liver (Gerhardt). Pleurisy with hæmorrhagic extravasation may occur in purpura, and is met with occasionally in cases of the acute exanthemata of a hæmorrhagic character.

Pleuritis may occur at any period of childhood, though in the primary form it is seen more rarely during the early than in the later years, and both sexes seem equally liable.

Diagnosis.-When the effusion is abundant, the disease may always be easily recognised by a careful examination and an accurate estimation of the physical signs; whilst the cases where it is more limited are much more difficult of recognition.

Diffuse pleuritis, with a copious exudation, may in children be mistaken for pneumonia, and the more easily if it occurs coincidently with croupous pneumonia (pleuro-pneumonia).

Hydrothorax, or passive dropsy of the pleura, differs from pleuritis with effusion in the variations of the level of the fluid, which agree with the alterations in the position of the patient, also by occurring usually on both sides, and in its course and in the causes to which it is due.

Prognosis.-In infancy this is generally more favourable than in later years, and in expressing an opinion attention must be paid to the form of the inflammation, the age of the child, and the exciting cause. The primary forms of the disease admit of more favourable prognosis than the secondary, especially those of a pyæmic and septic nature; and when there is a tendency to a purulent or tubercular transition, the disease is very serious and very often fatal.

Treatment.-At the outset of the disease a mild and restricted diet, with febrifuge remedies, such as digitalis, nitrate and acetate of potash, are necessary. For the relief of the stinging pain, repeated sinapisms are useful, and to mitigate the cough small doses of opium, Dover's powder, the extract of cannabis indicus, the aqua laurocerasi, and other like drugs may be given. When the fever is severe and there are reasons to suspect purulent exudation, quinine must be given in doses of half a grain to a grain, two or three times a day. When symptoms of suffocation occur from the extreme amount of effusion, the chest must be tapped without loss of time.

In chronic pleurisy the routine treatment consists of quinine 
and its preparations, iron and iodine, the use of whey diet and sorrel in summer, together with removal to pure air and restriction to a suitable diet.

If the pleurisy should become purulent and the symptoms become unfavorable, thoracentesis must be performed, though very frequently the natural efforts will create an exit. The cavity may afterwards be injected with iodine. 


\section{FOURTH DIVISION}

DISEASES OF THE ORGANS OF THE CIRCULATION AND OF THE LYMPHATIC SYSTEM

THE lesions which are found in the heart and its envelopes and in the great vessels may be divided into those which are congenital and those which are acquired. The gradual development of the heart from a simple cylinder into a hollow organ, with partition walls and apertures for blood-vessels, does not always proceed with regularity and completeness; for arrest and deficiency of development of the organ may occur in many different ways, to an extent which may render an independent life of the child altogether impossible, or at least interfere very materially with its health; though there may also be malformation of a kind which will exercise no detrimental influence. The transition from the congenital to the acquired lesions of the heart is marked by the continuance of some of the peculiarities of the foetal circulation, especially the patency of the ductus Botalli and of the foramen ovale, which are closed under the normal conditions of the pulmonary circulation. Some of the malformations have no clinical interest, their importance being merely anatomical; and such abnormalities as acardia or absence of the heart, double heart and ectopia cordis, and abnormal configuration or size of the organ, come under clinical notice rarely or never. It is otherwise, however, with such malformations as patency of the foramen ovale, or of the ductus Botalli, communication between the ventricles and auricles, and congenital stenosis of the pulmonary artery or aorta.

A frequent cardiac lesion in infancy is met with in hypertrophy without valvular lesion, the result of some protracted obstruction to respiration or to the circulation, as, for instance, in rickets, chronic bronchial catarrh, broncho-pneumonia, 
tuberculosis, typhoid, \&c. Careful investigation has shown that myocarditis and fatty degeneration of the muscular substance of the heart do not occur frequently in children. As a useful rule in diagnosis, it may be laid down that affections of the heart occurring in children under four years of age are generally congenital; and that only after that age do the acquired diseases make their appearance, for their chief exciting cause, rheumatism, is seldom met with in children under four years of age.

\section{Patency of the Foramen Ovale}

This seems to be due to a deficiency in the mechanism of its closure, in a too large size of the aperture or a too small size of its valve, in an unusually powerful bloodstream during fœetal life, and in other circumstances still obscure. If the foramen be not closed during the first week of life it usually remains patent for several years, or permanently. Sometimes patency of the ductus Botalli, or a communication between the ventricles, is formed coincidentally with a patent foramen ovale. As a rule, this condition is not characterised by any peculiar symptoms, and, if not complicated by some other anomaly, it is rarely diagnosed during life. Some few cases, as those recorded by Von Foster, and which were marked by peculiar circumstances, may be taken as exceptions to the rule.

\section{Patency of the Ductus Botalli}

The ductus Botalli, an important canal which shortens the route of the fortal circulation, is usually closed by the establishment of respiration, and becomes gradually obliterated, so that at the end of the third month it is represented by a shrivelled fibrous cord. As the result of pulmonary affections, especially atelectasis and some others not well known, the duct may remain patent, or may even be increased in size. When the patency is of limited extent there is usually no symptom, but when well marked it produces symptoms a short time after birth which may afford 
ground for a probable diagnosis. These are palpitation of the heart, breathlessness, slight degree of cyanosis, hæmorrhage from the nose and lungs, hypertrophy of the heart, and a systolic murmur heard over the origin of the pulmonary artery, but not constant. If the patency of the duct be complicated by patency of the foramen ovale, or the presence of a communication between the ventricles, the symptoms become complicated and are to be interpreted with caution.

Life may be continued for many years though the duct be pervious.

\section{Communication between the Ventricles}

Towards the end of the second or beginning of the third month of fœtal life the septum ventriculorum is usually so developed that the separation of the ventricles is complete. The perfect closure may, however, be prevented by an insufficient growth of the tissue of the septum, or by a disproportionate pressure of the blood current, and then will result a communication between the ventricles through an opening of greater or less size. This aperture is generally found at the membranous part of the septum, though it may also be found in the middle or some other part of it. Whether a destructive myo-endocarditis may re-open a septum already closed is a point not yet established; indeed, the cases where there is a communication between the ventricles seem due to an arrest of development.

Along with this malformation there may be found otherlesions, as hypertrophy of the heart, secondary endocarditis in one or both ventricles with exudation on the flaps of the mitral and tricuspid valves, patency of the foramen ovale or of the ductus. Botalli, or complete absence of the latter, stenosis of the pulmonary artery, or abnormal origin of the great vessels.

The clinical symptoms of this abnormality have not yet been sufficiently established to enable its diagnosis to be made with certainty; this being due partly to the simultaneous occurrences in the same heart of several malformations, and partly to the insufficient investigation of those cases in which the condition has been found. As somewhat constant symptoms I have 
noticed the following:-an increase of the cardiac dulness, especially in its transverse measurement, exaggeration of the heart's impulse between the fifth and sixth ribs, accentuation of the second pulmonary sound, a fremitus perceptible to the fingers, and on auscultation a systolic murmur, loudest towards the apex, and when there is at the same time absence of the valves, the murmur is peculiarly loud and extensive over the whole cardiac region, so that the heart-sounds are completely concealed.

Cyanosis may be more or less marked, though it may also be altogether absent. Its occurrence is due to a turgescence of the systemic veins and retardation of the capillary circulation at the periphery, in cases where the emptying of the various contents into the right side of the heart is hindered or rendered impossible. Palpitation of the heart, cough, shortness of breath, tendency to hæmorrhage, œdema and general dropsy, may be mentioned amongst the general symptoms.

Children with this malformation seldom reach the seventh year of life, but perish from valvular lesion or dropsy.

The treatment can be only dietetic and symptomatic.

\section{Congenital Stenosis of the Pulmonary Artery}

Like the other malformations of the heart this one may be found existing in different degrees, from slight narrowing of the pulmonary orifices to complete obliteration of the artery. The stenosis affects either the pulmonary artery or its conus, the former more frequently, and may generally be referred to intrauterine endocarditis or myocarditis. The previously mentioned malformations may be found as probable results of the stenosis, and in proportion to its severity.

The train of symptoms may vary, according to the extent of the stenosis and its accompanying lesions. The most constant and striking symptom is cyanosis, which usually is present at birth, and becomes more marked as life advances. The physical symptoms consist of increase of the cardiac dulness and exaggeration of the cardiac impulse, a tactile fremitus and a systolic murmur, most audible over the origin of the pulmonary artery. Among the general symptoms may be mentioned a 
tendency to hæmorrhage, deficient development, superficial and weak respiration, cough, attacks of suffocation, cold skin, giddiness and fainting. Tubercle of the lungs, especially of the left, is often found as a complication, and the disturbance to the returning circulation produces œdema of the brain, liver, spleen, kidneys, \&c. These symptoms, however, do not enable the diagnosis to be made with certainty, especially in complicated cases.

The prognosis is always unfavorable, the more so in proportion to the severity of the cyanosis, or with the occurrence of tubercle in the lungs or parenchymatous nephritis. As a rule, the patient dies asphyxiated soon after birth, or lives only until the period of the second dentition, adult life being very rarely reached. I have seen one*case in a girl sixteen years of age in whom the cyanosis was peculiarly well marked.

The treatment must consist of perfect rest from bodily exertion and freedom from exposure to cold, a mild nutritious diet, and such therapeutic means as may be indicated for the prophylaxis or treatment of any complication.

\section{Congenital Tricuspid Stenosis}

This is of much less common occurrence than stenosis of the pulmonary artery, and consists of complete or partial closing of the orifice by muscular or membranous bands. Intra-uterine endocarditis, or excessive development of the muscular substance, may be given as causes. In proportion to the degree of stenosis the right ventricle remains often markedly undilated, and patency of the foramen ovale and ductus Botalli, or a communication between the ventricles, are often present along with the stenosis. The chief symptoms met with during life in these cases are cyanosis, a troublesome dry cough, difficulty of breathing, with suffocative attacks, hæmorrhages, and dropsical effusions.

Physical examination shows an increase of cardiac dulness downwards and towards the left side, and a systolic murmur, which is widely distributed, but most pronounced towards the lower part of the sternum, though this latter sign may be absent. The diagnosis, however, is neither easy nor can it be made with 
certainty, and of all the symptoms the most trustworthy are the non-extension of the cardiac dulness towards the right side and the peculiar position of the greatest intensity of the murmur.

Children affected by this malformation generally die early.

\section{Congenital Aortic Stenosis}

As in the case of the tricuspid orifice, this may consist only of narrowing of the aortic opening, the contraction seldom extending up the tube, or there may be complete closure from intra-uterine inflammation of the cusps of the valves or of the inner coat of the vessel, or persistence of its original size may be referred to as a cause of the malformation. When the aortic orifice is completely occluded the child never lives long, and no indications for diagnosis can be given.

\section{Pericarditis}

Inflammation of the pericardium, to any great extent, is a rare affection in infancy, though it is not unfrequently met with in limited extent, exclusive of the milk spots so frequently noticed on the serous surface.

Anatomy.-The chief indications of the inflammation are hyperæmia, thickening and roughening of the surface of the epiand pericardium, with abundant plastic exudation in the form of net-like papillæ (cor villosum) or membranous folds; or the exudation may take the form of fluid with fibrinous flocculi, may be purulent, and occasionally is found to be tinged with blood. As the result of chronic pericarditis there are sometimes found layers of mortar-like substance, from two to four millimetres thick, or tubercular nodules. Partial adhesion and, in some rare cases, complete fusion of the two serous surfaces, hypertrophy, softening, inflammation, and fatty degeneration of the heart, are also found as the result of this disease.

Symptoms and course.-The symptoms are doubtful or sharply marked according to the extent of the inflammation and the quality of the exudation. More advanced children complain of oppression and pain over the cardiac region or scrobiculus cordis, 
and palpitation of the heart and dyspnœa are often met with, though sometimes they are absent. The pyrexial symptoms are not usually severe, unless they are due to the presence of some other affection, and the pulse is usually small and irregular, and beats from 120 to 140 in the minute. Besides these symptoms we often see cyanosis, œdema, diminished secretion of urine, anxious expression of face, with the features slightly swollen and blanched or cyanotic, an undulatory movement in the jugular veins, delirium, and a peculiar undescribable restlessness of the patient. The most important signs for diagnosis are an alteration in the outline of the cardiac dulness and the presence of a creaking or rasping murmur the result of friction. 'This friction murmur is neither so often nor so well heard in children as in adults, and in the former is always indicative of a fibrinous exudation; sometimes it may be felt by the hand, is usually not communicated, is neither systolic nor diastolic, but sometimes continuous, and is generally most audible towards the base or towards the apex. All these signs and symptoms are far from being constant in children, and may be either entirely absent or insufficiently developed when the exudation is small in amount, so that the diagnosis of pericarditis is often difficult and sometimes impossible in children.

When the inflammation is limited, recovery is the rule; but when the exudation is abundant, especially when it becomes purulent, death occurs almost always, and often with very unexpected rapidity. If adhesions are formed, more especially if they amount to complete fusion of the two serous surfaces, they sometimes lead to disturbances of the circulation, which continue for a long time, or may become permanent.

Endo- and myo-carditis often form complications of pericarditis, especially in the course of acute rheumatism.

Causes.-Pericarditis is most frequently observed in the course of acute articular rheumatism, with or without endocarditis and valvular disease; next in frequency during the course of pleurisy, pneumonia, the acute exanthemata, especially scarlet fever and smallpox, also in parenchymatous nephritis; and less commonly in tuberculosis, pyæmia, and purpura hæmorrhagica. In new-born children the disease is usually purulent and ichorous, the result of some septic process which often proceeds from the umbilicus, or of tubercle (F. Weber), and it is then generally com- 
plicated with pleurisy, meningitis, or peritonitis. It may occur during intra-uterine life (Billard). Primary pericarditis in childhood is a very rare occurrence.

Treatment.-Acute pericarditis, with more or less clear indications of sero-fibrinous exudation, demands mild antiphlogistic treatment, such as sinapisms or vesicants to the cardiac region, (cold packing has also been recommended); with small doses of digitalis and acetate of potash. If absorption is delayed diuretics are to be given, and absorbents, such as the preparations of iodine, applied to the skin over the heart.

If the circulation becomes impeded and the pulse becomes small, with dyspnœic symptoms, stimulants, such as wine, ammonia and musk, must be given freely. When the disease has taken the pyæmic form quinine and its preparations are used, though without much result, for it nearly always has a fatal termination.

\section{Dropsy of the Pericardium. Hydropericardium}

Dropsy of the pericardium is almost always a secondary affection, frequently associated with general dropsy or with serous effusion into the pleura or peritoneum. It is not so often seen in the course of heart disease in children as in adults; in the former its most frequent cause being parenchymatous nephritis, scarlet fever, the general dropsy which occurs along with chronic enteritis, tuberculosis, amyloid degeneration of abdominal organs, purpura, and hooping-cough.

Anatomically it is characterised by a quantity of clear yellow serum, varying from two to six ounces, and which has the chemical peculiarities common to all serous effusions. Slight serous infiltration of the pericardium and fatty degeneration of the muscular tissue of the heart, are often associated with the dropsy.

Symptoms and course.-Slight effusions give rise to no symptoms, but when the effusion is large, amounting at least to a few ounces, it gives rise to the general symptoms of pericarditis. The patient is more or less restless and affected by difficulty of breathing, which will be the greater if there should be present at the same time dropsy of the pleura and peritoneum; the 
semi-recumbent position is generally selected as the easiest, the skin is blanched and cyanotic, with a somewhat depressed temperature and slight œdema ; the pulse is small, frequent, and irregular, and the jugular veins distended. The cardiac region is somewhat prominent, the apiceal impulse is much diminished or may be entirely destroyed, percussion shows a notable increase of the cardiac dulness, and auscultation reveals that the heart sounds are dulled and weakened, but never the presence of any friction murmur. Epistaxis, headache, vomiting, and gastric irritability may be noticed amongst the general symptoms, especially if there be coincident disease of the heart.

Pericardial dropsy does sometimes, though rarely, result in recovery, but the general issue is a fatal one, and may be due either to the pericardial effusion alone, or to the combined influence of some other lesion.

Treatment.-This must be directed partly against the disease in which the dropsy has its origin, and partly against the local lesion. Diuretics such as digitalis, acetate of potash, and juniper are useful, and benefit may also be had by derivation from the skin and intestinal tract. If the breathlessness is very severe, stimulants, as wine, spirits and ammonia, form the most appropriate remedies. The operation of tapping the pericardium has not been largely adopted.

\section{Endocarditis and Valvular Disease}

This may occur during intra-uterine life, and have as its results some of the cardiac abnormalities which have been detailed above; or it may originate after birth in the same way as it does in adult life.

Anatomy.-The endocardium, which does not exist as a distinct membrane, but is continuous with the lining membrane of the vessels, gives indications in infancy almost always of the exudative form of inflammation, though in some rare instances the purulent and ulcerative variety is met with. The inflammation is seen either on the surface of the cavities or on the surface of the valves, frequently on both sites, and it may also extend outwards and involve the muscular tissue. When it affects the lining of the cavities, the resulting thickening and 
contraction of the papillary muscles and tendinous cords produce valvular incompetency. When the valvular cusps are attacked they lose their flexibility and become covered with vegetations composed of connective tissue, and these, along with depositions of fibrin, make the surfaces of the valves rough, and produce the different varieties of valvular disease, as incompetency or stenosis, with the resulting complications of hypertrophy and rupture of the muscular apparatus. By the dislodgment of some of these vegetations and their impaction elsewhere in the circulation, there are produced embolism and sometimes metastatic centres of inflammation in other organs, as the spleen, kidney, \&c., but rarely in the lungs.

Fœtal endocarditis occurs most frequently on the right side, especially at the pulmonary orifice; whilst the endocarditis of after life occurs generally on the left side, with a special preference for the mitral orifice.

Symptoms and course.-It may be stated generally that endocarditis and valvular disease in children present pretty much the same symptoms as they do in adult life, but that in the case of children the diagnosis is much more difficult. Endocarditis which does not affect any of the valves gives rise to no physical sign, and only to such functional symptoms as are rather uncertain, and it often remains latent. When the papillary muscles and tendinous cords are affected the indications of heart disease are somewhat delayed, but when the valvular surfaces are attacked the physical evidences of the lesion are immediate. Endocarditis in children usually has its seat on the left side, and especially often at the mitral valve. Incompetency is indicated by a murmur which is systolic, most audible between the apex and base of the heart, not communicated into the carotids, and intensified between the respiratory sounds. The right side of the heart is dilated, and the heart's impulse exaggerated and its area extended.

Stenosis of the left venous orifice, a rare lesion in children, and the result of endocarditis, gives rise to a diastolic murmur. When endocarditis affects the aortic valves the cardiac area of dulness is increased, and there is a diastolic murmur heard loudest at the base, or the murmur may be double.

Very rarely is the right side of the heart affected by endocarditis or valvular disease after birth, whilst the reverse is the 
case in intra-uterine life. Disease of the valves on both sides of the heart is very rare, and is usually of fotal origin, and the diagnosis is hardly possible.

As functional symptoms of endocarditis may be noticed some degree of fever, an expression of anxiety and restlessness, sense of oppression or pain over the heart, transient attacks of diffculty of breathing or even severe dyspnœa, dry cough, cyanosis, epistaxis, and unquiet sleep often interrupted by delirium. By embolic impaction encephalitis or capillary apoplexy may be produced in the brain, and circumscribed pneumonia in the lungs. The disease of the kidneys, the enlargement of the liver and spleen, and the areolar and saccular dropsies which are seen in adults as the result of heart disease, do not occur with the same frequency, and severity among children. Chorea minor is often seen as a complication of endocarditis in children, and is probably due to some localisation of the rheumatic poison in the spinal meninges.

Causes.-Endocarditis and valvular disease may be congenital, as has already been mentioned, though it is generally the result of an attack of acute rheumatism. In some exceptional cases it occurs in the course of some of the acute exanthemata, especially scarlet fever, and may also occur as a complication of pleurisy and pneumonia. Both the congenital and acquired varieties of heart disease seem to predispose the patient to recurrences of the endocarditis.

Prognosis.-As a simple acute inflammatory process enclocarditis is not very fatal, though by its complications and after results, especially consequent pericarditis, it is very often a serious clisease. Pre-existent valvular disease always darkens the prospects of the patient, though it must be borne in mind that the compensation gained in the cardiac action in some cases of valvular disease often mitigates its severity.

Treatment.-The treatment usually recommended for acute rheumatism does not seem to exercise any very distinct influence on the course of acute endocarditis. The patient must be kept absolutely quiet, and be liberally supplied with acid cooling drinks. The application of some form of counter-irritation to the skin over the heart, and the administration internally of small doses of digitalis and some calmative, such as the Aq. Laurocerasi, or when the disease is very acute, half grain or 
grain doses of quinine. Children with heart disease must be very carefully protected from cold and damp, as they have a ready aptitude for the occurrence of rheumatic attacks.

\section{Vascular Tumours, Angioma, Teleangiectasis}

These consist of an abnormal abundance and size of bloodvessels, are generally met with in the form of spots slightly elevated, and may be divided into two classes.

(a) The ragged angioma, teleangiectasis, or simple angioma of Virchow, is met with as spots or tumours of various sizes, bright red or bluish red in colour, consisting of connective tissue and fat inclosing a great number of adventitious blood-vessels of irregular form, and they are limited to the skin and subcutaneous connective tissue. These spots may either remain stationary or may increase towards the periphery; they become swollen and of a darker colour when the child cries or strains, and are diminished and blanched by direct pressure. The more deeply seated vascular tumours are soft, elastic and white, with a bluish translucency, or the skin may even be perfectly normal in appearance. Arterial angiomata are of a bright colour, somewhat exalted temperature, and distinctly pulsatile, whilst those of a venous origin are dark and do not pulsate. They are almost always congenital, and occur most frequently on the face and hairy scalp, though they are often seen elsewhere. To these belong the ordinary mother-marks, whether circumscribed or extensive. The ragged angiomata disappear sometimes spontaneously, also after suppuration and by atrophy and grangrene after inflammatory attacks. Hæmorrhage from them is not usually met with to a dangerous extent.

(b) The cavernous angioma, which does not often occur in children, consists of a stroma of connective tissue with abundant vascular supply entering a number of irregular cavities communicating with one another. It may be circumscribed or diffuse, and may be situated in the subcutaneous areolar tissue or deeper, even under the fascia, in the face, neck, extremities, trunk, bones or internal organs, as the liver. The tumours vary in size, and may be capsulated and circumscribed or diffuse, fluctuating or firm, sometimes painful, and the skin is sometimes thinned 
and discoloured, and in other instances quite unaltered. When they burst the hæmorrhage is always serious, sometimes fatal.

The ragged and the cavernous angioma may readily be mistaken the one for the other, especially in a case where the former extends deeply, but for assistance in the diagnosis the following points are valuable. The ragged angioma grows more rapidly than the circumscribed cavernous variety, is generally congenital, found only in children, and usually is painless; whilst the cavernous angioma is confined to no period of life, is very often painful, and is more readily emptied by the pressure of the finger than the other. Angiomata have been confounded with lipomata, cystic growths, cerebral hernia, and glandular tumours by very careful surgeons.

Treatment.-Spontaneous cure sometimes occurs, though only after the lapse of a considerable time; and in the case of a rapidly growing angioma, especially if there be any important organ in the neighbourhood which may become involved, destruction or removal of the tumour may become necessary. For spots and angiomata of small extent vaccination of the tumour or cauterization of it by sulphuric acid, or by chromic acid, especially the latter, is to be recommended; or the use of some irritant application such as diachylon plaster containing about 10 per cent. of tartar emetic. Angiomata of larger size demand the use of strong caustics, as the Vienna paste, or the galvanocaustic wire; but extirpation by the knife is always the quickest and surest remedy, though after removal of even small tumours formidable erysipelas may occur. On account of the violent hæmorrhage met with in the removal of cavernous angiomata, it is generally desirable to effect their destruction by means of the galvano-cautery, or by the actual cautery, or by ligature, galvano-puncture, or the injection of a diluted solution of the perchloride of iron.

\section{Inflammation of Lymphatic Glands, Lymphadenitis}

Diseases of the lymphatic glands in children form very common and important affections. Inflammation of these organs is generally chronic in its form and secondary in its relation, 
the cases where it is acute or primary being only rare. The anatomical results of this affection may be classed as resorption, suppuration, contraction, cheesy, tubercular and chalky degeneration, hypertrophy and amyloid and sarcomatous proliferation of the glandular parenchyma.

In simple inflammation the glands are increased in size, hardened, reddened, and infiltrated by serum, and in this stage recovery by resolution may take place. If the inflammatory process goes on to suppuration, abscesses are formed in which the surrounding cellular tissue is involved. Cheesy alteration of the gland-tissue is either the result of chronic inflammatory action, or of previous miliary deposit, and may affect the whole gland, or only part of it. It generally begins in the centre, seldom in the periphery of the gland, and results in softening of the tissue and the formation of a cavity, in which it is not unusual to find a free nucleus about the size of a hazel nut. Hypertrophy of the gland-structure may affect either the lymph follicles or the connective-tissue, in the latter case producing tumours about the size of a pigeon's to a hen's egg, often of a cartilaginous consistence (sarcomatous degeneration), and showing on section a regular fibrinous surface of a yellowish colour. A combination of several varieties is often found in the same specimen, and in the same child examples of the hyperplastic, suppurating, and cheesy gland may be met.with. 'The glands in the neck, the bronchial and mesenteric glands, are most frequently affected, and the relative proneness of these glands to the diseases is in the order in which they are given.

Symptoms and course.-These vary according to the form of the disease, its seat and extent, and they are also influenced by the idiosyncrasies of the patient. Acute glandular inflammation may run its course without any pyrexial symptoms," or fever may be present to a marked degree. In children who are very susceptible to morbid influences an acute glandular inflammation may induce serious symptoms, such as a pulse of $160-180$, and a temperature of $41^{\circ}$, with delirium and disturbed sleep. The glands are always increased in size, and sometimes, when several of those involved become confluent, they produce a tumour of considerable volume. According as the surrounding areolar tissue is involved or not, and in the usual course of acute inflammation of a gland the neighbouring tissue is 
generally affected, the tumour is more or less fixed. In acute inflammation the gland is always tender to the touch, but when the disease is chronic there may be little or absolutely no pain.

When a gland is affected by hyperplasia it is hard and resisting, but if suppuration occurs it becomes soft, and afterwards fluctuation can be felt. After the perforation of the skin by a gland which has suppurated or been affected by cheesy degeneration, there often remain ulcers and fistulous tracks of a very chronic and obstinate character, and these, even when they heal, produce very objectionable deformities. Pressure of the enlarged glands on the neighbouring blood-vessels often produce marked functional disturbances, the result of cerebral anæmia, such as giddiness, vomiting, or even epileptiform convulsions. Enlargement of the axillary or jugular lymphatic glands may, by pressure on the brachial nerves, lead to loss of sensation in the limb or to a distressing formication.

The clinical symptoms due to chronic broncho-adenitis and tuberculosis of the bronchial glands are very important; the larger the tumours are, and sometimes they are met with the size of a hen's egg or even the size of a fist, along the trachea and at the hilum of the lungs, the more marked the symptoms and the clearer their significance.

By pressure on the great vessels various disturbances in the circulation are brought about, as arterial anæmia, or passive venous hyperæmia seen in enlargement and distension of the superficial veins of the chest, forehead and temples. Thrombi are also formed sometimes in the jugular and innominate veins, though the vessels are not often actually occluded. The compression of the enlarged glands also often affects the lung-tissue, and the calibre of the neighbouring bronchi, and may even interfere 'with the trachea and œsophagus to the extent of perforation of the former. Pressure on the vagus sometimes gives rise to a peculiar spasmodic and periodic cough, occurring especially at night. The physical signs of enlargement of the bronchial glands consist of a distinctly limited dulness on percussion in the neighbourhood of the bifurcation of the trachea, an ill-defined inspiratory murmur with a sharp hissing expiration, resembling bronchial breathing, though by these no absolutely certain diagnosis can be made. 
A very distinct train of symptoms marks enlargement of the mesenteric glands (tabes meseraica), which will be found in detail in the chapter on abdominal diseases.

The most serious and fatal complication of cheesy or tubercular degeneration of lymphatic glands, especially of the bronchial and mesenteric glands, is the general infection they may give rise to. Tubercular meningitis, peritonitis, and general miliary tuberculosis are often excited by resorption of the tubercular matter, and their infection of distant parts.

Pseudo-leucocythemia and anæmia may also be mentioned as complications of this disease, due to very extensive alterations in the lymphatic glands and the spleen.

The duration of the glandular enlargement varies according to the nature of the exciting cause and the constitution of the child. In healthy, well-nourished children the glandular affections disappear more rapidly than they do in those who are weakly, scrofulous, tuberculous, or tainted with syphilis.

Causes.-Diseases of the lymphatic glands are very rarely primary in their origin, following usually in the wake of some other affection. These predisposing causes are of various kinds, and have their origin in different morbid centres. Diseases of the skin, especially of a chronic form, as eczema, impetigo, prurigo, furunculus, pemphigus, favus, \&c., excite enlargement of the glands in the neighbourhood, especially the glands of the neck when the scalp is the seat of the disease. Stomatitis and angina also produce a swollen condition of the submaxillary glands, and disease of the bronchial mucous membrane leads to an increase of size of the bronchial glands, and disease of the intestinal mucous membrane also affects the mesenteric glands. Extensive glandular affection is generally met with in all the acute and chronic diseases which affect, to a marked extent, the processes of nutrition or the condition of the blood, such as scarlet fever, variola, syphilis, and, above all, scrofula.

Treatment.-This may be directed either topically to the glands themselves, or may be general with a view of acting on the exciting cause, and in some cases the removal of this is followed at once by a disappearance of the glandular enlargement, but in others the disease is much more obstinate. If there be evidence of a scrofulous habit, the use of cod-liver oil, the preparations of iodine and iron, the milk-cure, and iodized 
and bromized mineral waters, as those at Hall, Krankenheil, and Kreuznach are indicated. If there be a syphilitic taint, iodine and mercurials form the appropriate remedies. If the glandular enlargement be due to some skin eruption, that must, if possible, be cured, and so with any intestinal mischief of which there may be evidence. In some cases of acute glandular inflammation cold packing is very useful, and to stimulate absorption inunction by warm oil and mercurial ointments, and painting the glands with iodine, have been recommended. When suppuration of the gland has become certain, poultices are the best application. When the condition of the gland is doubtful, some inactive treatment, such as painting with tincture of iodine diluted with tincture of galls, or the application of some emollient plastęr, is advisable. Single enlarged glands often disappear under long continued pressure exercised by a pad. Indurated glands which obstinately resist all medicinal treatment may be removed by operative interference, though this is a proceeding not without risk, especially in the region of the neck. Injection of tincture of iodine, or a solution of iodide of potassium into the tissue of the gland, may stimulate its absorption or excite suppuration. Glandular abscesses may be opened either by incision or by the insertion of a seton, though I cannot say that the latter method obviates the objectionable scars which result from the incisions. Fistulous tracks should be laid open, and indolent ulcers are to be touched with caustic or sprinkled with calomel. When the presence of enlargement of internal glands is suspected, the preparations of iron, iodine, and quinine are the best remedies; and in all cases of glandular disease the diet must be carefully attended to, and the patient be removed, if possible, to country air.

\section{Anæmia lymphatica, pseudo-leucocythemia, adenia}

Pseudo-leucocythemia, first recognised and described by Hodgkin, is not a very uncommon disease. The most important and striking symptoms are a peculiar and strongly marked blanching of the skin which seems almost transparent, and an increase in size of both superficial and deep lymphatic glands; 
and in many cases the liver and spleen are also enlarged (anæmic spleen-tumour).

The patient presents all the functional disturbances of severe anæmia, becomes weary and exhausted on the slightest exertion, and suffers from palpitation of the heart, a tendency to hæmorrhage, breathlessness, and often from œdema of the extremities.

In one case, a girl eleven years of age, I saw this disease begin with typhoid symptoms, followed after a time by general glandular enlargement, the latter condition alternately improving, and getting worse for about two years; after which she recovered completely. The blood usually shows no increase in the number of white cells, or only to a slight extent; but the hyperplastic glands are seen to have the connective-tissue elements greatly increased. In the brain there is sometimes found, but not always, an albuminous substance resembling peptone, precipitable by alcohol. The course of this disease may vary in duration from a few months to several years. Death from general exhaustion is the usual ending of it, recovery being very exceptional, though I have seen one case, as already mentioned.

'The affection may be confused with true leucocythemia, and scrofulosis or sarcosis of the glands, though the former may be easily discriminated by a microscopic examination of the blood.

The causes are still unknown, though I have seen cases where it might have been referred with probability to the influence of rickets and chronic intestinal catarrh. The treatment consists of the administration of iron, quinine, and cod-liver oil, good nourishing diet, country air, and removal to a fresh residence. Iodine must be used with caution, and only in combination with the remedies already mentioned. 


\section{FIFTH DIVISION}

\section{DISEASES OF THE ORGANS OF DIGESTION}

\section{Preliminary Observations on the Nourishment of Children}

For the proper growth of children during the first year of life it is absolutely necessary that their natural nourishment should be sufficient both in quantity and quality, and any defect in either may be followed by the most serious results. There can be little doubt that the high rate of mortality during the first year of life can be, to a large extent, explained by errors in the nutrition of the infants.

The best and safest nourishment for a child is always the milk of its own mother, and no woman who is able to undertake it should neglect the sacred duty of suckling her own offspring.

According to the investigations of Becquerel and Vernois, the milk of a healthy woman has the following composition:

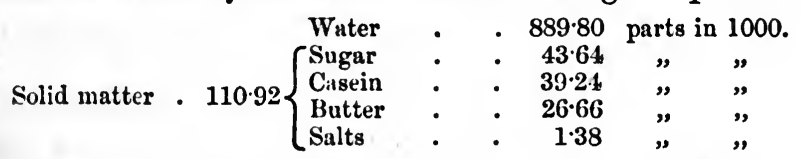

The specific gravity of fresh human milk is 1.032 , its colour blueish or clear white, its taste slightly sweetish and insipid, and its reaction alkaline. When the milk is stale, or when the mother is suffering from disease, the reaction becomes acid, and in this condition the casein is deposited in small knots or flakes, as is seen in curdled cows' milk where the casein separates in large clots.

Towards the end of gestation, and during the first few days of lactation, the chemical and physical condition of the milk differs from what it is afterwards in that it is yellower, thicker, 
richer in solid matter, especially in fat and salts, and on microscopic examination the colostrum corpuscles are seen, which have a measurement of $\cdot 006$ to .023 of a line (Henle); whilst the so-called milk-globules (milchkügelchen), which are only the epithelial elements of the gland in a state of fatty degeneration, have a measurement of $\cdot 0012$ to $\cdot 0020$. The colostrum communicates to the milk a slightly laxative action, which assists the evacuation of the meconium.

There are various diseases, either of a temporary or permanent character, which may so affect the quantity and quality of the milk as to prevent the mother suckling the child. Thus, mental disturbance, as fear, anger, sorrow, pain, \&c., often make the mother's milk thin, deficient in sugar, and too much like whey, and may thereby excite sudden attacks of vomiting, diarrhœa, or even convulsions. The quantity of sugar in the milk diminishes in proportion as time elapses after the confinement of the mother.

The occurrence of menstruation diminishes the amount of organic matter in the milk, and generally. excites in the suckling more or less severe gastric disturbances, even though it may not produce any chemical alteration in the milk. If these derangements of digestion of the child should not continue after the period of menstruation, there : is no, reason for advising the removal of the child from the breast; but if they do, then some change must be made in the child's nourishment.

Another pregnancy occurring during the period of lactation generally, though not always, makes the milk poorer, and causes a reappearance of the colostrum corpuscles. But even should this impoverishment of the milk not be sufficient to require that lactation should be discontinued, yet in the interest of the child, the mother and the fœtus, it should be forbidden. A moderate amount of sexual intercourse does not seem to exert any prejudicial influence on the character of the milk.

Whilst there are many mothers who suckle their children with advantage, yet there are numbers, and they are on the increase owing to the fashion of our social customs, who either cannot, dare not, or will not rear their own young. In some congenital deficiency of development of the breasts and consequent small supply of milk, and in others diseases such as 
inflammation, suppuration, induration, or cancerous degeneration of the gland, act as preventives. When the nipples are small, flat, and retracted, or affected by painful and bleeding fissures, it is often a difficult matter for the mother to nurse, yet the nipples may be so improved by careful preparation previous to the birth of the child, that the mother may be able to suckle it. ${ }^{1}$ Certain conditions of life and occupation make it impossible for the mother to suckle her child, or at least to do so regularly.

Sometimes the impediment to the nursing exists in the child itself, as double harelip and cleft palate.

When the mother is affected by such diseases as tubercle. scrofula, gout, chronic and inveterate skin eruptions, or epilepsy, she should not •be allowed to suckle her child; but transient illness, such as typhoid fever, dysentery, pneumonia, the acute exanthemata, rheumatism, \&c., are not absolutely prohibitory. I often permit mothers suffering from typhoid or dysentery to continue nursing without any subsequent detriment to the child. Should the milk disappear entirely, or to a considerable extent, during the disease, then of course the child is to be taken from the breast.

Finally, the mother may withdraw herself from her duties as a nurse from shame, or from some more culpable ground of convenience, or from a desire to $\mathrm{mix}$ in fashionable frivolities or dissipation.

The time and manner of nursing are very important, and on these points many errors are committed. During the first few days after birth, when the stomach cannot assimilate any large quantity of food, the child may be put to the breast very frequently, but afterwards this must be done with a certain degree of regularity. When the child is healthy, and the quantity and quality of the milk suitable, the child should have the breast every two hours during the day, and about every three hours during the night. The breast should not be regarded and used as a means of quieting a crying child, unless the cry-

1 My friend Professor Kehrer, of Giessen, showed me a neat little operation suitable for such cases, and which, in his experience, has been of much service. He removes a small ring of tissue from the areola, close to the nipple, and so makes the latter more prominent.-('Centralblat. f. d. Med. Wissenschaft.' 1873.)-L. T. 
ing be due to hunger, but the real cause of the restlessness should be sought for. For the reasons already given the child should never be allowed to suck immediately after the mother has suffered from mental excitement or severe bodily exertion, and in nursing both breasts should be used with a certain regular alternation.

The question of how long a child ought to be suckled depends for its answer on so many points belonging both to the nurse and to the child that no definite rule can be laid down. It may be said that generally it is advisable to continue the child at the breast till the occurrence of dentition, or till the complete appearance of the first group of teeth, or, on the average, to the ninth or tenth month. Sickly and rachitic children require to be kept longer at the breast than those which are healthy.

Children should not be weaned too soon nor suddenly, nor during teething, and, if possible, not during the extreme heat of summer. The preparation for weaning should occupy several weeks or months, consisting in the administration of some artificial food to the child, and may begin between the third and fifth months. What this artificial addition may best be cannot always be predicted, and it is often necessary to discover what is most suitable by experiment. Diluted beef tea, rusk, meal, or groats cooked in milk (pap) will all generally be readily taken by children, and will suit them very well. When the weaning of the child has to be done suddenly, as may happen from some acute disease affecting the nurse, the child almost always suffers to some extent; indeed, often is the subject of dangerous or even rapidly fatal disease.

If the infant's own mother either cannot, dare not or will not nurse her child, the best substitute is a wet-nurse; but even this involves so much risk that great caution is necessary in the selection of one. This is no place to discuss the moral questions involved in the emplnyment of wet-nurses, for the advantage gained by it in the great majority of cases dismisses any objections to our using a means by which human life may be saved.

A good and useful nurse should have the following properties:- She must have good bodily health, be free from communicable diseases, and her breasts ought to be firm, elastic, 
not too fat, and provided with nipples sufficiently prominent and free from excoriations; and it is advisable that a careful examination should be made of her complexion, gums, teeth, lymphatic glands of the neck, and the genitals. The best age for a wet-nurse is between twenty and thirty, and women are better for the purpose who are of slender build than those who are fat and lusty. There does not seem to be any advantage in fair-complexioned women over dark, or vice versâ, but those from the country are to be preferred to those from town, and they are better if they have had some previous experience in nursing children.

The milk of the nurse must be sufficient in quantity and quality, and the best test for this, though not an absolutely certain one nor always practicable, is the examination of her own child, which ought never to be omitted. The evidence of an abundant stipply of milk is a better guarantee of the goodness of a wet-nurse than any chemico-optical examination of the milk. It is a somewhat important matter that the dates of the several confinements of the mother and the nurse selected should not be very far apart, so that there should be but little difference between the age of the milk and the age of the child to be nourished by it. This difference should not exceed six weeks. 'The best test of the efficiency of the milk is given by the child thriving on it or not, and by the condition of its digestion.

The nurse should, if possible, be good tempered and of a mild disposition, though if we remember from what class of the population wet-nurses are generally taken, we cannot afford to be particular on this point if the child thrives. The well-known unscrupulousness of nurses, still more of those who procure and hire them, must make us very cautious in the selection of one, and be on our guard against imposition; and especial care must be taken to ascertain by some trustworthy evidence the date of the nurse's confinement, if her child cannot be seen.

In those cases where the mother cannot nurse and where a wet-nurse cannot be obtained, there still remains the plan of rearing on artificial food. The importance of this question has led to many attempts to find some substitute for the mother's milk. Formerly, these attempts were only empirical, but of late they have been made on a more scientific basis, and the labours of Liebig have been especially fruitful in this direction. 
But by whatever means the artificial rearing be conducted, it requires great skill and care.

The best substitute for human milk is undoubtedly that of some animal, and on account of the readiness with which it can be obtained the most convenient is cow's milk. It has a very close general resemblance to human milk, though it contains less water, butter, sugar, and free alkali, but more casein and salts, and has a slight alkaline reaction, becomes easily sour when the casein separates in curd, and its digestion by the child is rendered more difficult. The condition of the milk depends very much on the food of the cow, and that brought from the country is, therefore, always to be preferred. The value of cow's milk as a substitute for that of the mother is, therefore, very much diminished in large towns, owing to the various adulterations it undergoes, some of them made with substances which exercise very deleterious influences on the children. A strict sanitary inspection of the milk trade, under the direction of the police, could do much to restrain the evil produced by this iniquitous adulteration. In order to resist the tendency milk has to become sour, especially in summer time, it is advisable to render it faintly alkaline with bicarbonate of potash.

During the early months of infancy the child should have the cow's milk somewhat diluted, at first two thirds milk to one third of water; in the second month only a fourth of water need be added, and after the fourth month the pure milk may be given. The milk must always be slightly warmed, from $37^{\circ}$ to $38^{\circ}$ Cent., before being given to the child, and the best apparatus for its administration is a feeding bottle with a nipple-shaped mouthpiece of vulcanite or decalcified ivory, its convenience being much increased by the addition of an elastic tube, about six inches long, between the bottle and the mouthpiece. Great care must be taken to keep all the parts very clean.

The artificial food must be given at regular intervals, and, when possible, it must be made fresh each time, especially when it consists of some of the substances about to be mentioned.

The readiness with which cow's milk decomposes, and the adulterations to which it is liable, have induced many attempts to find some other food for children, and these have led, 
amongst other things, to the introduction of condensed milk and Liebig's food for infants. Of the former there are two preparations, that of an American Company known as the Anglo-Swiss Condensed Milk Company, at Cham, in the Canton Schweiz, and that of the German-Swiss Milk-Extract Company at Vevey, Schweiz, and Kempten, Bavaria.' 'The condensed milk can be recommended as a valuable substitute for fresh cow's milk for the nourishment of infants, even though it only has the relation to the latter that the dried herb has to the fresh and living plant.

Liebig's food for infants, the result of scientific investigation, resembles the mother's milk in that the plastic (nitrogenous) and heat-producing (non-nitrogenous, respiratory) ingredients are present in it in the same proportions as they exist in the milk. Extracts of Liebig's food are prepared by Ed. Lofflund, Roth and Braun, J. Paul Liebe, \&c.

According to my own experience, which has been pretty considerable, Liebig's food is generally found to be easily digested and of pleasant taste, and answers all the requirements of artificial nourishment for infants, though it also is open to the objection common to all substitutes, that in some cases it either cannot be taken, or does not agree with the child, exciting vomiting and diarrhœa, and in this case must be mixed with pure, unadulterated milk.

There are various other substances which may be used either as substitutes for, or aids to, cow's milk, such as the various kinds of coffee, pap, and beef tea.

Amongst the first may be mentioned acorn coffee, cocoa nibs, Loschner's coffee for infants (prepared by Tschenkel \& Son in Lobositz), barley coffee, and the ordinary Mocha coffee. Of the paps there are those prepared from wheat, rye, rice, potato meal, infants' groats, arrowroot, rusks (of which those prepared by Wafflers, of Nuremberg, are to be especially recommended), and white bread mixed with milk, water, or beef tea.

Beef tea or chicken soup, when given to an infant, should be made without salt or vegetable, or at first even slightly sweetened with sugar, and not very strong; and it may have a

I In this country there are the Aylesbury, Mallow, and Crosse \& Blackwell's condensed milk, but of all that I have tried the Aylesbury milk is by far the best for infants' food.-L. T. 
little milk added to it, or be slightly thickened with rice, fine groats, sago, oatmeal, barley, or white of egg.

Beer may also be tried either in its ordinary condition or better, boiled and slightly sweetened, for sometimes it will be found to suit children with weak digestion better than either milk or beef tea. No practitioner should allow any child under his care to be dosed with any of the numerous quack preparations, whether of a nutritive or sedative character. In many cases several of the varieties of artificial food will have to be tried before that which is suited to the particular child will be found, and all the functions must be considered in order to attain success. Thus, if the child has cut some teeth, a little meat may be given, either pounded or chopped very fine. Children of two years old and over may be allowed to join in the meals of their parents if there be a judicious selection in what they eat, so as to exclude anything that is difficult of digestion, strongly seasoned, very fat, heating or stimulating. A very reprehensible fashion has of late years grown up of feeding children almost exclusively on meat, especially of giving it to them for supper. For the latter meal children should have only broth, vegetables, light farinaceous food, fruit, \&c.; and the neglect of this rule has doubtless something to do with the anæmic tendency of the present generation.

The best drink for children is pure water, though they may be allowed to have a little sound beer mixed with it, especially if they are weakly.

\section{A. Mouth and Throat}

\section{Narrowing of the orifice of the mouth, microstomia}

This condition is sometimes acquired, sometimes congenital, for we find many children with a disproportionate smallness of the mouth, or even with complete adhesion of the lips (atresia oris), and in others the diminution in size or contraction is due to croupo-diphtheritic or syphilitic ulceration, some gangrenous attack (noma), or to burns of the lips.

The deformity may come on gradually, from the contraction 
of a cicatrix, the original injury of which was not of importance.

As a preventive measure, it is very important to treat all ulceration of the lips with great care, and to obviate any misplaced adhesions. For its treatment, microstomia admits only of some surgical proceeding, such as the chyloplastic operation of Dieffenbach, though after these operations children often die of atrophy.

\section{Harelip and cleft palate}

Of the malformations of the mouth these two deformities are the most important. Harelip may be single, with a cleft on one side of the middle line corresponding to one nostril, or double, with a cleft on either side, between which is a flap of skin varying in size and breadth. Sometimes the lip is intimately attached to the gum, and when the cleft extends through the gum and jaw, it is known as cleft palate. The cleft in the jaw passes between the incisor and canine teeth, and may pass backwards, or even on both sides, to a varying depth. The simple cleft in the gum may be of only slight extent, from half a line to a line in breadth, or may be very wide, presenting a complete chasm extending into the posterior nares. The cleft also often extends into the soft palate, and the intermaxillary bones may be found well developed and large, placed underneath the nose as prominent nodules, or they may not be disproportionately increased in size, and the cleft simple. ${ }^{1}$

The functional disturbances produced by this malformation

The varieties of cleft palate seem to vary in frequency in different districts. In the northern and midland districts of England the most common by far is the simple cleft of the soft palate, extending only slightly forwards into the palate bones, and without harelip. Next in frequency is a more advanced palatal cleft, with harelip. Next the complete cleft, and least common of all is the double cleft, with protruding intermaxillary bones.

That geographical situation has much to do with this deformity in the human species I am quite certain, and in the lower animals it is proved by the fact that all lion whelps born in the London Zoological Gardens have cleft palate, while in other gardens this deformity is unknown. In some districts of Yorkshire cleft palate would seem to be endemic, and that there it is not merely a family peculiarity is seen by its appearance in the children of immigrants, in whose families it was previonsly unknown. It is markedly atavic.-L. T. 
vary with its extent, the most important being due to the diffculty the child has in sucking.

A child with simple harelip may be able to attach himself to the breast, and be suckled in the usual way, but in cases of cleft palate it is almost always necessary to feed the child by the spoon or bottle.

Intestinal catarrh and atrophy generally carry these latter malformed children rapidly off, and if they live they are afflicted with an indistinctness and difficulty of speech. If the child be not operated on before the appearance of the first teeth, or if the operation be not successful, the face becomes very much deformed from the oblique position of the teeth.

The cause of this deformity is an arrest of development during fœtal life.

Treatment.-The only method of cure is by operation, and the result of that is not always successful. Surgeons differ in opinion as to the time of election for the performance of the operation. For most reasons it is advisable not to perform an operation for harelip until the child is four or six weeks old, and when the fissure is double the same rule holds good, with this addition, that both fissures should not be closed at once, but with an interval of three or four weeks between them; as in this way there is greater chance of success. When there is cleft palate as well, it is best to operate on the lip first at about the fourth week, and after that to attempt to close the palatal cleft.

The cleft, if left alone, will become narrower as the development of the child advances, on which account it is advisable to delay the operation for some time, especially if the child is going on satisfactorily. If the soft palate alone is cleft it may be closed by sutures, and if the hard palate be affected, the aperture may be closed either by the use of an artificial obturator or by an operation, though the general experience is that this operation has more failures than successes. ${ }^{1}$ The proceedings appropriate to these cases are to be found described in various surgical works.

1 This qualified recommendation of the operation for cleft palate is, I think, scarcely sufficient after the satisfactory results obtained by Sir W. Fergusson, Mr. T. Smith, Mr. G. Pollock, and myself, ('Brit. and Foreign Med.-Chir. Review;' 1870.)-L. T. 


\section{Ranula}

Any cystic formation which is contained in the anterior angle of the lower jaw in the sublingual space is included in the term ranula, such formations being, in the majority of cases, nothing more than a dropsical distension of the sublingual salivary gland (Fleischmann), or of its ducts. The tumours vary in size from that of a pea to a walnut, are round, soft, elastic, transparent and fluctuating, are of very slow growth, and their contents are somewhat viscid, gluey, and transparent, of a slightly yellow colour, and composed of water and albuminates, especially the albuminate of soda. In some exceptional cases the contents are pultaceous, resembling the products of atheroma, and then the tumour is only slightly transparent, and does not fluctuate. As the cyst grows the tongue is pushed more and more upwards, the jaw pressed outwards, the breathing, sucking, and swallowing much interfered with, the voice rendered indistinct, and if the tumour be allowed to reach a large size it may even give rise to symptoms of suffocation.

Prognosis. - This is always favorable. Sometimes the tumour undergoes a spontaneous cure by inflammation and suppuration of the cyst, but, as a rule, it remains stationary after it has reached a certain size.

Treatment.-The radical cure is a surgical proceeding by which a piece of the cyst wall is removed, and its interior cauterised by nitrate of silver or stuffed with charpie soaked in perchloride of iron; or by splitting the cyst and enucleating it. Phisick, Longier, and Guersant have recommended the use of setons. Simple puncture is of little use, as very soon the cyst refills and gains its original size.

\section{Anchylo-glottis; tongue-tie or tongue-tack}

In new-born children there is often found, and indeed sometimes also both in boys and girls of more advanced age, a degree of shortness of the frxnum of the tongue which constitutes a deformity, the protrusion of the tongue and the act of 
sucking being more or less impeded. Sometimes the deficiency of mucous membrane is so great that it passes at once from the floor of the mouth on to the tongue without the formation of any frænum. When the frænum is too short it may be snipped with scissors, though even this simple operation must be performed with care, as I have seen two cases where incisions made too deep have resulted in fatal hæmorrhage. Cases of delayed speech in children three or four years old are often explained by non-medical persons on the ground of tongue-tie, the real origin of which lies in deaf-mutism, in chronic hydrocephalus, or in disturbed or delayed development of some special part of the brain, probably at the Sylvian fissure.

\section{Catarrh of the mouth; stomatitis catarrhalis, S. simplex, S. erythematosa}

Hyperæmia of the mucous membrane of the mouth (erythema), and a higher degree of the same, as catarrhal inflammation, are frequent affections of children, occurring generally as secondary, rarely as primary lesions. The anatomical changes in simple erythema consist of alteration of the colour of the mucous membrane to a dark red, sometimes limited to isolated and circumscribed spots, and in other cases extending over large surfaces or uniformly over the whole membrane, which is then swollen and dry, the papillæ of the tongue enlarged and injected, its epithelium abraded, and the mucous glands of the lips and cheeks projecting as whitish-grey, yellowish, or transparent nodules, and yielding on pressure a drop of mucus.

Along with the swollen mucous glands there are sometimes seen small vesicular patches, resembling herpes, which soon dry up and leave scales behind them. The epithelium of the tongue is abundantly shed, and the buccal secretion is increased in quantity, is of acid reaction, sometimes thin, sometimes viscid and flocculent, and seems to produce excoriations at the angles of the mouth and on the chin where it runs over. The temperature of the mouth is perceptibly raised, and considerable pain is felt throughout the cavity, but neither the secretion nor the ulcers give rise to any special offensive smell. Sucklings affected by catarrhal stomatitis do not readily take the breast, but prefer 
cold drinks, and they offer resistance to any disturbance or examination of the mouth.

This discase may arise from the use of food or drinks which are too hot, or acrid and irritating, from tecthing, a carious tooth, want of attention to cleanliness, and the use of certain drugs, such as mercury, iodine, antimony, and arsenic. It may also arise in the course of certain pyrexial diseases, especially some of the acute exanthemata, such as typhoid, measles, and scarlet fever, and in Bright's disease. Catarrhal stomatitis, also, may be met with in the first stage of other and more serious inflammatory and ulcerative diseases of the mucous membrane of the mouth.

Treatment.-The first care must be to discover, if possible, and remove the cause, for it often happens that the simple increased attention to the cleanliness of the mouth, the removal of the sordes, the eutting of one or more teeth, or the removal of one that is carious, suffice completely to effect a cure. Sometimes a simple wash for the mouth, containing borax or chlorate of potash, suffices, or if there be ulceration the surfaces may be touched with nitrate of silver, though this is very painful, and has no very apparent effect in shortening the disease. When there is a very abundant flow of saliva it is always advisable to protect the chest from the constant moisture by a bibb of oiled or vulcanised cloth.

\section{Aphtha; stomatitis aphthosa}

In this disease the anatomical alteration consists of circumscribed spots of inflammation, either not raised at all or only slightly so above the level of the healthy mucous membrane,yellow or yellowish-white in colour, with a margin of red surrounding them, and covered with exudation of fibro-cellullar material situated beneath the epithelial layer and the corium. These spots are generally round, vary in size from a pin's point to a pea, sometimes streaky, and are situated on the mucous membrane of the lips, cheeks, tongue, gums, and even the tonsils. Their number may vary very much, there being sometimes only two or three, sometimes many more, especially on the edges of the tongue and on the tonsils. These spots of exudation run 
their course very rapidly, and disappear usually without leaving any ulcer or cicatrix; though sometimes the exudation seems to be somewhat deeply seated in the superficial corium, and then it leaves behind shallow excoriations, which heal rapidly. Sometimes the exudation is a result of some previous process, for it is seldom absent when there is an abundant secretion of fluid from the mouth with an acid reaction, accompanied by pain especially when food is taken. The disease may or may not be ushered in by symptoms of fever, but after the appearance of the exudation the fever may be very severe, and be accompanied by symptoms of considerable irritation.

Aphthæ are seldom idiopathic, though in this form the disease may constitute an epidemic, but usually they are secondary and symptomatic, occurring in the course of dentition, intestinal catarrh, acute zymotic diseases such as measles, smallpox, scarlet fever, typhoid, and diphtheria; also in pneumonia and hooping-cough. It occurs most frequently in children between six and thirteen months old.

There is no difficulty in the diagnosis of the disease if the spots are situated in the front of the mouth, though when on the throat, roof of the mouth and tonsils, they may be mistaken for follicular tonsillitis or diphtheria.

Treatment.-Recovery usually takes place in a few days, though sometimes recurrences may delay it for ten days or a fortnight, and the intervals between the remissions may sometimes occupy some days or even a week. No remedies yet tried have seemed to exercise much influence on the course of the disease. Aqua calcis, chlorate of potash, and more astringent substances, such as alum, zinc, \&c., may be tried as local applications ; but the application of nitrate of silver is not advisable unless the disease is of a chronic nature. If there is much fever or constipation, antiphlogistic, refrigerant or slightly aperient remedies are to be given.

As a variety of aphthous stomatitis there may be mentioned the aphtha of new-born infants, described by Bednar, which is met with as yellowish or slightly grey spots, of the size of a linseed to that of a split pea, slightly raised above the surface, and which may give place to an ulcer or may disappear without any trace. It is seen in infants during the first weeks of life, and makes sucking painful to them. A wash for the mouth con- 
taining lime water, chlorate of potash, or borax, is the best treatment.

\section{Croupous inflammation of the mucous membrane of the mouth; stomatitis et angina crouposa}

In this disease the mucous membrane of the mouth and throat is of an intense red colour and much swollen, and is scattered with fibro-cellular membranous masses, which may be removed without destruction of the mucous membrane. This exudation may have only one occurrence, or it may be found several times in succession. Its usual seat is on the gums, tonsils, and more rarely the tongue, lips, cheeks, and hard palate, and in some cases the whole mucous surface of the mouth and throat is occupied by this membrane and croupous laryngitis, bronchitis, pneumonia and diphtheritis are frequently met with as complications of this disease. The general symptoms consist of pain and difficulty in swallowing and breathing, more or less pyrexia, and great restlessness, to which may be added those of laryngeal croup when the affection is extending downwards.

Croupous stomatitis may be either an idiopathic or a secondary affection, in the latter case being often met with in connection with some of the acute exanthemata. In the diagnosis there is little difficulty in the presence of the peculiar exudation, which must be distinguished from that of diphtheria. 'The treatment of this disease very much resembles that of diphtheria.

\section{Diphtheria; angina diphtheritica}

Diphtheria often presents itself as a sequence of some other disease generally of the zymotic type, and in that case is very often of epidemic occurrence, but in other instances it is idiopathic and purely local in its seat. Pathologically it frequently cannot be distinguished from croup, both being often enough met with together, and neither the seat nor the kind of exudation afford anatomical grounds for separating them. It is, however, desirable. if possible, to separate them clinically. 
The mucous membrane becomes hyperæmic and swollen, and there appear on it punctate or roundish spots of exudation of white, yellowish white, or distinctly yellow colour, the product of a fibrinous parenchymatous inflammation; and this, by compression of the capillaries, may result in death of the superficial or even deeper parts of the mucous membrane and be thrown off either in its membranous form or as purulent débris. After its removal the mucous membrane is seen intensely congested, thickened, bleeding, and suffering from loss of substance to a greater or less depth. The abundant formation of pus corpuscles on the mucous surface, or even in the submucous tissue, may lead, in the more severe class of cases, to ichoro-purulent or even gangrenous destruction of the soft parts, followed, in cases of recovery, by cicatricial distortion. The masses thrown off in such cases are of a dark brown colour, from the admixture of blood, and ecchymoses are often seen between the affected spots.

The most usual seats of the diphtheritic exudation are the tonsils, uvula, and soft palate, the posterior wall of the pharynx, and the posterior nares; but it may extend into the nostrils, the larynx the trachea, and stomach, and may appear in the mucous membrane of the genitals in girls, on the conjunctiva, and on any wounds of the skin.

Symptoms and course.- Diphtheria sometimes occurs as a mild, comparatively harmless and completely localised affection; but, on the other hand, it may be severe, intensified by complications, and result in fatal issue. In the former class of cases we see the children affected by only a slight degree of fever, or almost entirely free from it, suffering from slightly disturbed sleep and headache, with a wearied expression of face, a sense of burning in the throat, especially in swallowing, and on one or both tonsils whitish-grey spots of exudation, which gradually become confluent and are thrown off in two or three days, leaving the subjacent tissue hyperæmic, bleeding, and ulcerated. As the feverish symptoms and subjective disturbances disappear, these ulcers rapidly heal, the neighbouring lymphatics are only slightly affected, and in eight or ten days the whole course of the disease comes to a fortunate termination. Occasionally this slight form is ushered in by very severe general symptoms, such as high fever, violent delirium, vomiting and great restlessness, which disappear, however, on the second or third day with the 
appearance of the exudation, and the further course of the disease is mild. The general and local lesions may in some cases be so mild that the children are never confined either to bed or even to their rooms, but complain only of being slightly indisposed, and continue going about.

Patients suffering from diphtheria in the severe form have a very different train of symptoms, elicited either by the extension of the diphtheritic inflammation into vital organs or by the induction of blood-poisoning, both of which conditions involve very great risk to life. The initial symptoms may be slight, and may not attract much notice, as in the mild form of the disease; or the symptom-complex may be of a severe character from the outset, and there may occur simultaneously or in rapid succession such symptoms as vomiting, violent headache, general depression, rigors followed by high fever, convulsions, constipation, and loss of appetite. At first the throat is only reddened and dry, but patches of diphtheritic exudation soon appear, which are at first isolated, but afterwards assume greater dimensions and affect the whole of the cavity. This is accompanied by pain and difficulty in swallowing, regurgitation of the food and drink through the mouth and nose, an indistinct nasal voice, and an abundant secretion of a thick, viscid fluid, which is extremely distressing to the patient. The ichorous discharge, or gangrenous destruction of the soft tissue, communicates a very offensive carrion-like smell to the breath.

The lymphatic glands in the neighbourhood of the diphtheritic exudation usually become swollen on both sides, rarely only on one, and this in mild cases is limited to a simple hyperplastic increase in size, but in the more severe cases the ichoro-purulent infection produces inflammation of the glands, which goes on to suppuration, and, by the absorption of the septic material, may lead to general infection of the system. The urine is diminished in quantity, and often, though not constantly, it contains albumen; while blood, fibrinous and epithelial tubecasts, are now and then, especially in epidemics, met with, indicating the occurrence of parenchymatous nephritis, and accompanied by symptoms of uræmia.

Invasion of the nasal cavities by the diphtheritic inflammation is indicated by epistaxis and by a discharge of an ichoropurulent fluid which excoriates the nostrils. The substance of 
the lower eyclid is also sometimes affected, and so is the ear, the latter complication being indicated by pain in the ear and purulent discharge from the meatus.

When the disease advances into the larynx, which it seldom does immediately but only after a few days from the beginning of the disease, the well-known symptoms of exudative laryngitis are met with, though the occurrence of a dry, barking cough must not always be taken as indicative of this serious condition, as it may arise from simple catarrhal swelling of the laryngeal mucous membrane. In some cases the symptoms of laryngeal stenosis are due to œdema of the glottis.

If the inflammation extends into the œsophagus and stomach there may be no distinct clinical evidence of it, though under such circumstances there is often frequent and obstinate vomiting, unquenchable thirst, and rapid collapse.

On the skin of children suffering from diphtheria there is often seen a transient and widely diffused erythematous rash, resembling scarlet fever, which occasionally goes on to the formation of pemphigoid vesicles, or even hæmorrhagic petechiæ.

Occasionally we meet with cases of diphtheria sine diphthera, that is, cases of simple reddening of the fauces without any perceptible patches of exudation, though with the fever and the symptoms of intense nervous depression. In some instances where several members of the same family have been laid up with diphtheria I have seen these cases.

Diphtheritic septicæmia is marked by severe rigors, increase of temperature, small weak pulse, bluish-yellow or earthy colour of the face, dull and sunken eyes, a completely apathetic condition of the child, absolute loss of appetite, and frequent bleeding at the nose; and when these symptoms occur in the course of the disease they have a very unfavorable significance, as they generally precede death. The occurrence of the septicæmia in cases of diphtheria generally takes place a few days after the commencement of the disease, and originates in the absorption cf the matter from the affected lymphatic glands.

Anæmia and a peculiar paralysis are often seen as the sequelæ of an attack of diphtheria; the former appearing towards the end of the attack sometimes in the form of toxic anæmia, with a peculiar wax-like blanching of the skin, weak and irregular pulse, general dulness and depression of spirits. This condition 
generally results in complete recovery in a few weeks, though it may also end fatally by the occurrence of convulsions and loss of consciousness.

The diphtheritic paralysis is usually developed somewhat considerably after the remission of the acute symptoms. Its immediate source of origin can, from our present knowledge, only be suspected to be probably the diphtheritic alteration of the blood acting on the peripheral or central nervous mechanism. According to Buhl, there occurs a diphtheritic infiltration of the sheaths of the nerves.

The most common scats of the paralysis are the throat, eyes, and extremities, especially the lower, the muscles of the trunk, larynx, bladder, and gut being rarely involved; and it may be simple or complex, or it may be incomplete with fecble movements and diminished sensibility, or complete with absolute loss of motive power and perfect anæsthesia. According to the constancy of their seats, the following varieties of paralysis may be noted, with their symptoms :-When the palate and muscles of swallowing are involved, there is difficulty of swallowing, sucking, and expectoration, together with an alteration of the voiee of a peculiar nasal character. When the ocular muscles are affected there is loss of accommodation, and in the less frequent implication of the abducent nerve we have:squinting, diplopia, dimness of vision, and flashes of light before the eyes. There is no apparent change in the eyes themselves. When the paralysis attacks the laryngeal muscles the voice loses its resonance or is completely lost, and there is a loss of power to expectorate or cough strongly; when the superior laryngeal is paralysed, the muscles which move it allow the epiglottis to lie back against the tongue, and this condition, along with the anæsthesia of the mucous membrane, may allow of the entrance of foreign substances, and produce sudden and fatal suffocation. In such cases laryngoscopic examination shows relaxation of the vocal and ventricular cords.

Paralysis of the limbs, muscles of respiration, bladder, and rectum, will be found referred to under their proper section.

Some cases of mental disease, as mania or dementia; have been noticed as occurring after an attack of diphtheria (Ehrle, Foville, \&c.):

Causes.-Diphtheria may be at its onset either a local affec- 
tion, and may run its course as such, or may become an affection of the general system by the absorption of the septic material; or it may be from the first a blood disease, resembling the other zymotics. Pathologically, it is characterised by a croupoparenchymatous inflammation of the mucous membrane, with resulting necrosis of the same; and though organisms of a low type have been observed in the product of this inflammation, and also in the blood of the affected patients (Hueter, Hallier, Letzerich), yet we cannot regard these as the cause of the disease, for they are more probably the result of it.

Diphtheria may appear in a primary form, and as such it is often epidemic, or it may be secondary to some other acute or chronic disease, such as scarlet fever, measles, smallpox, typhoid, hooping-cough, scrofula, and other exhausting diseases of children. Prolonged residence in crowded hospitals, beyond a doubt, sometimes excites secondary diphtheria in children. The disease occurs in children of all ages, but is least common in sucklings ; certain families seem to have an hereditary disposition for it, and an irritable condition of the throat seems to predispose certain individuals towards it. I met with a case recently where the diphtheria was secondary to scarlet fever, and ended fatally from the blood-poisoning; in which the diphtheritic exudation was confined to the left tonsil, which was much enlarged, and from which it disappeared; whilst the right tonsil, which had been in great part removed a few days before, remained unaffected.

Diphtheria is contagious, both in the sporadic and epidemic form, but the medium by which the infection is conveyed is not known, though it is certain that prolonged stay with the patient is sufficient for it. And the disease is met with in the wellappointed houses of the rich as well as in the miserable dwellings of mendicants. One attack does not seem to confer future immunity from the disease, as I have had cases under my care where there have been two attacks. The period of incubation is variously given by different authors, my own experience leading me to believe that it is from two to sixteen days, and from Trendelenberg's experiments with rabbits it would seem to be from two to three days.

Diagnosis.-When the disease is at all well marked the diagnosis is very easy, and it is scarcely possible to mistake it 
for anything else ; but in the milder forms it may be mistaken for angina crouposa (Flächencroup), angina aphthosa, tonsillitis folliculosa and phlegmonosa, or even for syphilitic sore throat. A careful investigation of the diphtheritic patches, however, and a microscopic investigation of them, will prevent such mistakes. True croupous exudation is free and membranous, whilst the diphtheritic is parenchymatous, with necrobiosis. When the general symptoms are very well marked, and those of the throat only slight, an inspection of the latter will sometimes yield decided evidence of the nature of the case; and at times when diphtheria is known to be epidemic, the throat of every child attacked by acute symptoms should be examined.

Diphtheritic paralysis has sometimes been mistaken for the early stage of hip-joint disease, but a careful inquiry into the history and course of the case will obviate this mistake.

Prognosis.-This must always be cautious, for even very slight local symptoms afford no guarantee of a fortunate issue of any case; and the danger increases in proportion to the extent of the exudation, especially when the larynx becomes implicated, for of such cases about three fourths die. When symptoms of gastritis, pneumonia, pulmonary gangrene, or nephritis or blood-poisoning occur, the prognosis becomes correspondingly unfavorable, and it is also influenced in this direction the younger the patient. The death-rate varies, according to the character of the epidemic and the ages of the children attacked, from 30 to 60 per cent. Relapses, or the occurrence of inflammation of the glands of the neck, are to be regarded as unfavorable signs.

Diphtheritic paralysis admits of a much better prognosis than diphtheria itself, as most of the cases result in recovery.

Treatment.-As a prophylactic measure, it is always advisable to remove children not affected from a locality where an epidemic is raging.

The treatment of a case of diphtheria may consist either only of local applications, in the event of the disease not affecting the whole systen, or of combined local and general treatment when the contrary is the case. The object of local treatment is to cut short the exudation and the necrosis, and to prevent the absorption of the prisonous matter, and for this purpose the best remedy, according to my experience, is lime water. This 
may be employed, as a rule, either in its ordinary strength, or diluted with from two to four times its bulk of water, and, according to Kuchenmeister, its efficacy is increased by dilution. Or it may be used by means of the spray apparatus at intervals of every two or three hours, and A. Weber, of Darmstadt, has recommended the use of lactic acid in the same way every hour, in a solution of fifteen to twenty drops to the ounce of water, but it does not seem to be so efficacious. A solution of perchloride of iron, from a scruple to half a drachm of the salt to two ounces of water, has also been recommended for pencilling the fauces, as has also spirit of wine diluted with one part of water. Sulphur has been recommended by Barbarosa and others, administered by insufflation of flowers of sulphur in very fine division, but it does not seem to have any specific action on the diphtheria. Ice is very useful in relieving the symptoms, given in small pieces every few minutes, or the ice-water in teaspoonfuls to children who are too young or wilful to take the solid ice. The use of disinfecting gargles, when there is fœtor from the decomposition of the tissues, is often found to be beneficial, and of these the best are chlorate of potash, two drachms to six ounces of water; permanganate of potash, in a solution of one to twio grains to the ounce of water; chlorine water, half a drachm to the ounce; or carbolic acid, in the proportion of one or two grains to the ounce. Active cauterization of the affected parts with nitrate of silver, muriatic or chromic acids, also the inhalation of bromine, have been found to be of only doubtful service, and are not much to be recommended.

The internal treatment must consist of quinine, chlorate of potash, and the administration of stimulants, should there be any indication of failure of the vital powers. When there is epistaxis, the liquor ferri pernitratis must be applied.

When the larynx becomes implicated, the various external and internal remedies, which have already been referred to under the diseases of the larynx, such as emetics, must be employed; and, failing any benefit from these, there remains only the operation of tracheotomy.

The paralysis resulting from attacks of diphtheria often dis. appears without treatment; but if recovery be delayed, the preparations of quinine and iron, abundant nourishment, and removal to country air, may be made use of to hasten it. 
Severe cases of diphtheria, especially if complicated or occurring in severe epidemics, too often resist all remedies, and it is not unusual to see all the children of a family swept off in a few days by this terrible disease.

\section{Stomatitis ulcerosa}

This is a somewhat common disease amongst children, and consists of parenchymatous gingivitis, with ulcerative destruction of the mucous membrane. At first there is seen a marked reddening and swelling of the gingival mueous membrane, which is either. general or, less commonly, limited to isolated and circumscribed patches; and this swelling increases, so that the margins of gum between the teeth stand out like flasks, and they are so soft and tender that they bleed on the slightest touch. Soon the margins of the gum in contact with the teeth become altered in colour to a yellow or yellowish-grey; their tissue becomes softened and undergoes a pultaceous change, which gradually extends deeper and wider, and results in the formation of superficial ulcers. The teeth, deprived of their covering, become loose and easily removed from the alveoli, and the process of chewing is so painful that it is avoided by the patient. The flow of saliva is increased, and there is a peculiar smell of the breath, generally present from the very commencement of the disease, which is noticeable at a considerable distance, and is very characteristic of the disease. The submaxillary glands and the lymphatic glands of the neck are generally swollen, and there is often œdema of the face, limited, perhaps, to the side affected. 'This disease rarely limits itself to the gums, but generally extends to the lips and mucous membrane in contact with them, or to the tongue, producing ulcers, which not infrequently correspond with the impression of the teeth. Sometimes the periosteum of the jaw is destroyed, and necrosis of the bone results, and this has been seen to so severe an extent as to involve half the jaw ; and in the cases of a few weakly and badly nourished children the stomatitis passes into actual gangrene or noma. The original seat of the ulceration is usually the jaw, more frequently the lower than the upper, and the implication of the general system is, as a rule, but slight, and when there is much pain it is generally due to 
some complication. Its course is sometimes very acute, but, on the other hand, it is often very chronic, remaining stationary for a long time, after having reached a certain point.

Causes.-These are to be attributed partly to local conditions, and partly to the general condition of the child. It is never found in infants previous to dentition, though $I$ have seen it in some seven or eight months old, in whom only two incisors had appeared, and the great majority of the cases occur between the ages of three to eight years. Gums which are soft, loose, and affected by chronic hyperæmia are more apt to be affected than those which are red, firm, and closely applied round the teeth; and sickly, rickety, or scrofulous children are more readily affected than those that are healthy. Its occurrence is undoubtedly favoured by residence in unhealthy, cold, and damp houses, in badly ventilated hospitals or boarding-houses, also by insufficient nourishment; and it is peculiarly apt to occur during convalescence from some severe diseases, especially typhoid, measles, scarlet fever, smallpox, dysentery, \&c.; from difficult dentition, and, in cases of scorbutus, from the careless administration of preparations of mercury and lead, and in phosphorus poisoning.

Stomatitis ulcerosa is not contagious, though it is sometimes seen in an almost epidemic profusion.

Treatment. - This must consist chiefly in efforts to counteract the predisposing cause, by removal to better sanitary conditions, if these be defective, and by the administration of stimulant tonics; and in the local application, either by gargle or brushing of chlorate of potash, which seems in this disease to surpass all other remedies. In the case of necrosis great care must be taken for the cleanliness of the mouth, and poultices are to be applied. When the disease has progressed into noma, necrosis almost always results, and nothing seems to have much influence over it.

\section{Gangrene of the mouth; noma}

Fortunately, this disease is not very common. Its point of origin is not always the same, though generally it begins on the buccal surface of the cheek, less frequently on one of the lips; and it first appears as a yellowish vesicle, about the size of a 
linseed or a pea, which rapidly extends into an ill-coloured spot of gangrene. In other cases this initial vesicle is not seen, but the noma begins as a hard swelling in the soft tissues of the check, which rapidly becomes necrotic; and only exceptionally it has its origin in ulcerative stomatitis, or in an external ulceration of the skin of the cheek. From whatsoever point it starts, it rapidly increases in extent and depth; the lips, cheeks and chin become odematous, the skin tight and glistening, and at the point affected of a dark livid colour, this decolorisation gradually spreading into the healthy tissues, from which it may be either sharply defined or merged into them by an intermediate zone. It not uncommonly happens that the child dies in this stage, but if this should not happen the gangrene results in perforation of the tissues, and the cheeks, lips, nose, and even the eyelids and tissues in the neighbourhood of the ear, may be involved, and the bones exposed.

Noma most frequently attacks the cheeks, and the left somewhat oftener than the right, the chin seldom, and the female genitals may also be affected. Although generally unilateral, it may be met with involving both sides, as I have seen in one case, where the disease destroyed tissue to the extent of a halfcrown piece symmetrically near each ear.

At first the general symptoms are slight, or there may be none at all, the child seldom complaining of pain, continuing to have a good appetite, and persisting in its amusements; and as the vessels are closed at a very early stage, it very seldom happens that there is any hæmorrhage, even when the destruction of the tissues is very great.

On the other hand, however, the patient may, from the onset of the gangrene, present symptoms of depression, severe fever, and even delirium. In the further course of the disease exhausting diarrhœa, intense depression of the vital powers, small and frequent pulse, and cold, clammy skin, are amongst the most prominent symptoms; and as complications of the noma, I have met with broncho-pneumonia, pulmonary gangrene, hæmorrhagic erosion of the stomach, ichorous dysentery, secondary hydrocephalus, and Löschner records the occurrence of thrombosis of the cerebral sinuses.

The disease is generally fatal in from three to fourteen days, and, if it should result in recovery, after the arrest of the 
gangrene and the reparation of the slough, the loss of substance delays the healing, and the cicatricial contraction is always troublesome.

Causes.-The greater number of cases occur within the first six years of life, the disease being more frequent in girls than in boys, and almost always met with in children who are weakly, ill-housed, and ill-nourished, being almost unknown amongst the children of the well-to-do. In the great majority of cases it is of secondary origin, following other diseases, such as measles, typhoid, chronic intestinal catarrh, ulcerative stomatitis, scarlet fever, smallpox, broncho-pneumonia, tuberculosis, intermitting fever, hooping-cough, scrofula, and the administration of large doses of mercury; and the order in which these causes are given may be taken to represent the frequency of their action in exciting noma.

Spring and autumn are the seasons which seem most to favour its production. The discase is not contagious, yet it is an advisable precaution to isolate patients affected by it.

Prognosis.-This is very unfavorable, for recovery is really very exceptional. Out of 102 cases in my own experience, only four have got well, and of these three had it after typhoid and one after scarlet fever; in three it affected the face, and in the fourth the genitals were attacked.

Treatment.-On the whole this is very unsatisfactory. .. In the early stage cauterization by means of strong muriatic or sulphuric acid, or by nitrate of silver, may be had recourse to, and any slough removed by scissors; the ulcers should be frequently wetted with a strong solution of chlorate of potash (two or three drachms to eight ounces of water); permanganate of potash or chlorinated lime, and pads of lint soaked in the solution should be left in contact with them. Some disinfectant, such as a solution of creosote of half a drachm to a pint, will be found of advantage by destroying the foul smell. Strong nourishment, eggs, meat, wine, and beer, with perfect rest, and, if possible, fresh air, constitute the best general treatment; but regard must also be had to the exciting cause, and the practitioner must be on the watch for complications.

In cases of recovery the loss of tissue and the resulting cicatricial deformity may generally be in great measure repaired by a plastic operation. 


\section{Parasitic aphtha; stomatomykosis (Mehlmund)}

This affection of the mouth is not uncommon in sucklings, and is due to the development of a vegetable parasite. The mucous membrane is of a darkish red colour, somewhat dry, and at first is scattered with whitish points, which increase more or less rapidly in number and size, either to the formation of island-like masses or to a uniform thickish membrane, thickened into knots here and there, and felted in filiform layers.

These masses are white or whitish yellow, or, in children affected by jaundice, of a very intense yellow, are not unlike coagulated milk, and ar found chiefly on the lips, cheeks, gums, tongue, and folds of the mucous membrane. In very severe cases they extend into the cavity of the throat as far as the epiglottis, and even down the œsophagus into the stomach, as I have seen in several cases.

Recent observations have contradicted Reubold's statement that these masses are only pavement epithelium; but the observation of Zenker, who found the fungoid growths in the brain of a child in whose mouth they also were, has not yet been confirmed. These masses can be removed from the mucous membrane with more or less ease according as the fungoid fibres have invaded more or less intimately the vessels and superficial tissue.

The masses which have been described consist of epithelium and the filaments and spores of Oidium albicans, described and investigated by Berg, Gruby, and Robin. On microscopic examination are to be seen numerous and irregularly developed fungoid fibres, with laterally branching arms and buds, between which are placed round or oval sporules. Numerous fibres of the Leptothrix buccalis have also sometimes been found (Quinquand and Hausmann).

A short time before the appearance of these masses, or simultaneously with them, there is seen a marked reddening of the mucous membrane, the mouth becomes tender, and the child does not retain its hold of the nipple for long. The secretion of the mouth becomes markedly acid, and there is often a strongly acid smell from the mouth. When the masses spread into the 
throat and œsophagus swallowing becomes difficult, in extreme cases impossible, and the voice of the child becomes whimpering and hoarse. In the cases which I have met with, when the stomach was involved, there was frequent vomiting, but the occurrence of diarrhœa by the swallowing of the fungoid growth is not yet established.

Acute and chronic gastric and intestinal catarrh form frequent though not constant complications of this condition of the mouth, especially in children reared on artificial food; so also does aphthous stomatitis, and especially the gum-aphtha described by Bednar.

In well-nourished healthy children this parasitic affection of the mouth seldom lasts more than a few days, but in those who are weakly and ill-nourished it may become chronic, and may last weeks or even months.

Causes.-The method of the production of the fungoid growth has not yet been made clear, the majority of authors concur in attributing it to the formation of lactic acid by the decomposition of the food, though the observations of Berg, Ritter, and others, on the reaction of the saliva, do not support this view, and my own experience is against it, for in many cases I have not found acid reaction when the growth has been present.

Hausmann explains its occurrence by direct inoculation of the mouth from the passages of the mother, in the discharges of which he has found in 10 per cent. of all cases to contain forms resembling closely the parasites in question.

There can be no doubt that the disease may be communicated from one child to another by the nipple of the nurse, or by spoons or the mouthpiece of a feeding-bottle.

The disease affects sickly and ill-nourished children more readily than those which are healthy and well fed, and it is especially often found in those affected by scrofula and cachectic diseases; also in cases of typhoid. In one case of a boy suffering from caries of the spine I saw the aphtha return again and again at intervals of some months, and it always affected chiefly the dry, clammy, and intensely red mucous membrane of the tongue.

Treatment.-Great care must be taken to secure perfect cleanliness of the child's mouth, and to correct any error there may be in the general nutrition of the child, and if there be any 
fungoid appearance of the nipples of the nurse these must be attended to. For local application to the mouth of the child the best remedies are diluted lime-water and a solution of borax of the strength of a scruple or half a drachm to the ounce of water.

\section{Dentition and its dangers}

Like all other physiological processes, dentition may go on without any disturbance at all, or with very little, though in some cases its effect on the system is very serious, and may even destroy life.

There are certainly some pathological conditions which are intimately associated with teething as their cause, though this explanation of them must always be received with caution, and the fashion of attributing to dentition all diseases which may occur during its process must be carefully avoided.

The appearance of the twenty milk-teeth, as those of the first dentition are termed, does not take place in an uninterrupted sequence, but in a series of groups, separated by intervals of weeks or months. The following is the usual order and time of the cutting of the several groups:-

From the fifth to the seventh month, the two central lower incisors.

From the ninth to the eleventh, the four upper incisors, the two central appearing first.

From the thirteenth to the fifteenth, the two lower lateral incisors and the four first premolars.

From the sixteenth to the twentieth, the canines, those in the upper jaw generally preceding the lower.

From the twentieth to the thirtieth, the second premolars.

Many divergencies from these rules will be met with, as children may be born with one or more of their teeth already cut, or the teeth may appear earlier than usual or out of the usual order. In some families the upper incisors always appear before the lower, or one of the molars or the canine teeth precede the incisors, but these irregularities are of no importance. Delayed appearance of the teeth is, however, of greater moment, for it generally indicates some fault in the nutrition or a rickety habit of body. In the case of a boy who had well- 
marked rickets, and who was under my own observation, the first teeth did not appear till he was four years old.

With the appearance of the true molars, generally during the fifth or sixth year, the second dentition begins, the milkteeth disappearing in the order in which they came, to make way for the permanent teeth. The third molar, known also as the wisdom tooth, is late in being developed, and generally does not appear till between twenty and twenty-four years of age.

Dentition gives rise to two classes of affections-those which are local, and those which are sympathetic or reflex. The most important are the following:

Stomatitis, more usually the catarrhal form than either the aphthous or ulcerative, the symptoms of which have been detailed; and along with it sometimes occurs enlargement of the submaxillary and the lymphatic glands of the neck, seldom ending in suppuration.

Ulcers of the frænum linguæ are often seen at the time of cutting the first lower incisors, about the size of a linseed, surrounded by a reddened ring, and leaving greyish-yellow bases. These disappear in eight or ten days, often pass unnoticed, and are not to be compared with the traumatic ulcers occurring at the same place in the course of hooping-cough.

Febrile symptoms, with an increased temperature of the skin, exaltation of the pulse, and reddening of one or both cheeks, often accompany the process of dentition; but they are transient, like all the trouble of this physiological change, and have no typical character.

Certain nervous symptoms are also common, such as broken sleep, restlessness, crying and whimpering, slight spasms of the muscles of the face and extremities, or even eclamptic convulsions of a more severe kind.

Diarrhœa is a very common symptom in teething children, and may be due either to some affection of the mucous membrane of the mouth or to some reflex nervous action. When slight and transient, it scarcely requires treatment, but when persistent, more especially when the nature of the evacuations becomes altered, it must be very carefully attended to, for it must be borne in mind, first of all, that diarrhœa in a teething child unay be a symptom of some very scrious condition, and that, 
secondly, by inattention it may form the prelude to follicular enteritis.

Teething is so often accompanied by cough, that " teethingcough" has become a popular expression; and when this symptom is simply reflex in its origin, it is not accompanied by any alteration in the pulmonary mucous membrane and, therefore, is not accompanied by any auscultatory signs. It may, however, be very persistent and troublesome.

Many children suffer from skin eruptions whilst cutting their teeth; indeed, in some families all the children suffer in this way, and some children have a fresh attack as each set of teeth is cut. These generally take the form of erythema or urticaria, less frequently lichen or eczema, and must be considered as of vaso-motor origin.

Disturbances of the urinary system are sometimes seen, either as incontinence, occurring in children previously quite free from it; or in very frequent micturition, the child passing a quantity of clear urine of low specific gravity every ten or fifteen minutes, or, on the contrary, of spasmodic retention of urine. As these symptoms are always reflex, they last only a short time, from two to three days, and then disappear without any ill result, to return again, perhaps, when some more teeth are being cut.

I have seen catarrhal ophthalmia recur several times in children with the cutting of each group of teeth, and have evidently some connection with the process. This seems especially apt to occur when the eye tecth are being cut.

It must not be forgotten, in the consideration of any of the conditions above referred to, that the period of dentition also includes many other important changes, such as the weaning of the child, peculiar growth of the brain, and the time when rickets first make their appearance, so that great discrimination is necessary to place upon each its proper value.

Treatment.-In general this requires to be merely expectant, with a careful supervision of the child's diet and the removal of any source of excitement. . Slight diarrhœa needs no treatment, but if it becomes frequent, and the stools are watery and slimy, and if it be accompanied by vomiting, appropriate treatment must be had recourse to. Constipation should be relieved by clysters or slight aperients ; and for restlessness or sleeplessness and the other nervous symptoms, warm baths and the 
application of vinegar, diluted mustard, or infusion of horseradish, to the skin of the legs and feet will be found useful.

The treatment of convulsions will be found detailed elsewhere. Teething-cough, like the cough of pregnant women and chlorotic girls, usually defies all treatment, but disappears rapidly after the teeth have been cut. Formerly, the gums of nearly every child suffering from any of the maladies of dentition were cut, but this practice is falling more and more into disuse.

\section{Tonsillitis, angina tonsillarum}

Besides the aphthous, croupous and diphtheritic forms of inflammation already referred to as affecting the tonsils, these glands may be attacked by a simple inflammatory action, either confined to the follicles (angina follicularis), or involving the whole of their structure (angina phlegmonosa).

Symptoms and course.-The initial symptoms of this disease consist of shivering, fever, vomiting, epistaxis, sneezing, and, in young children, even delirium, though in some cases none of these symptoms are met with. The patient soon complains of a feeling of burning and dryness in the throat; swallowing becomes difficult and painful; there is an increase of the secretions of the mouth, an indistinct nasal tone of voice, noises in the ears, a difficulty in hearing, and stinging pain up towards the ear. In the more severe cases, especially when both tonsils are affected, these symptoms become very intense, the child is restless, excited, will not lie still, is troubled by difficulty of breathing, and may even be in danger of suffocation, which it expresses by seizing its neck as if it wished to remove some obstruction. In these cases fluid taken into the mouth is returned by the nose, and the enlargement of the tonsils may be felt outside under the lower jaw.

On examining the throat, in cases of follicular tonsillitis, one or both glands are found somewhat swollen, and spotted with reddened patches corresponding to the follicles, which in severe cases exude pus, and the exudation becoming hard, is loosened and coughed up. In the phlegmonous cases the tonsils are of an intensely dark red colour, much swollen, so as nearly to touch one another, and thereby almost close the isthmus of the fauces. 
In three or four days the swelling may pass off, and the tonsils gradually resume their former size, but in some cases the decrease only goes to a certain extent, and then, becoming indurated, the tonsils remain in a state of chronic enlargement. In children the inflammation seldom goes on to suppuration, but when it does, the pain and the difficulty of swallowing and breathing are very great, and the abscess usually bursts under the influence of some effort in coughing or vomiting.

I have seen a tonsillar abscess in a child only three weeks old, and in some cases both tonsils have suppurated, though this is very rare.

Causes.-The disease is almost peculiar to the later years of infancy, very rarely occurring in children at the breast, and it affects the two sexes equally. There can be little doubt that there exists in certain families a hereditary tendency to it, but it generally has an idiopathic origin in cold and in rapid changes of temperature, the latter influence producing it sometimes in almost epidemic frequency. It is also met with as symptomatic of other diseases, as scarlet fever, smallpox, hooping-cough, \&c.

Treatment.-The milder cases get rapidly well without any treatment, but when severe symptoms are seen at the beginning of a case, an abortive treatment may be attempted by pencilling the tonsils with a strong solution of nitrate of silver, a drachm or four scruples to the ounce of water, or with diluted Iiquor Ferri Perchloridi. Solid nitrate of silver should not be used in children, especially when they are very young. Children, as a rule, cannot use gargles, but, if old enough to do so, either lime water, a solution of alum, chlorate of potash, sal ammoniac, or the perchloride of iron, either in water or in some mucilaginous vehicle, will be found very useful. The inunction of the neck by oil, or the application of bacon rind, are popular external remedies, and a cravat wrung out of cold water and wrapped round the neck, has been highly recommended. When suppuration is threatening, emollient plasters or poultices will afford relief. Scarification of the tonsils can seldom be accomplished in children, and had better be avoided, but the use of ice by sucking it in small pieces will be found to give great relief, especially in the first stage. General symptoms require their appropriate treatment. 


\section{Hypertrophy of the tonsils}

The extent of the hypertrophy may vary very much in different cases, but the glands always present a ragged appearance and dark red colour, the surface marked by large vessels, and occasionally they are as large as a pigeon's egg, nearly touching in the middle, blocking up the passage to the pharynx, and encroaching on that cavity. The enlarged glands are firm or soft and more or less easily torn, according as their follicles or the connertive-tissue is chiefly implicated.

The functional lesions are partly mechanical and local, and partly due to the state of the general nutrition, these being very slight in the milder cases, but when the enlargement is great they may be very serious. From the diminution of the passage into the pharynx both respiration and swallowing are impeded, the former generally being passed through the mouth, and being accompanied during sleep by loud snoring. There is generally some pain in the ears and difficulty of hearing, and the voice is nasal in character. In extreme cases there is often epistaxis, an anxious expression of the face, with blanching or cyanosis, abnormal action of the muscles of the chest, and narrowing of its walls (Pectus carinatum). Hypertrophied tonsils are always apt to suffer from attacks of acute inflammation.

Towards puberty this affection will often disappear of itself, or it may remain stationary and the symptoms increase in severity. Enlargement of the tonsils is often congenital, especially in children born of parents tainted by scrofula or syphilis. It is in many cases the result of acute recurrent glandular inflammation, or of the angina of scarlet fever, diphtheria, \&c.

Treatment.-If there be any constitutional taint suspected as the origin of the tonsillar enlargement, such as scrofula, it must be treated by general remedies, such as cod-liver oil, iron, and iodine. The local treatment may consist of the application of caustics and astringents, as alum, nitrate of silver, tincture of iodine, powdered iodide of potassium, or the injection of chromic acid into the tissue of the gland (Lewin). The external application of tincture of iodine below the jaw has seemed, in several cases under my care, to have been beneficial. 
When the symptoms demand more active treatment the partial removal of the enlarged glands is the best remedy, an operation which is difficult in proportion as the child is young. It may be performed either with a single vulsellum and scissors or probe-pointed bistoury, or by the tonsil guillotine. Guersant has had a successful issue in a child so young as eighteen months, but, as a rule, it must not be lightly undertaken, but retained for the severest cases when there is danger to life; for the efforts of nature, aided by topical and general therapeutics, generally succeed in curing this disease, though the cure may be very gradual and protracted. As a prophylactic against the tendency to recurrence of acute tonsillitis, I know nothing so useful as daily gargling the throat and sponging the neck with cold water.

\section{Retro-pharyngeal abscess}

Collections of matter in the posterior and lateral walls of the pharynx are not very common, but when they do occur they are seen as flattish-round or oval swellings varying in size, are seldom idiopathic, but are generally due to caries of the cervical vertebræ or to pyæmic infection. They have also been met with as the result of the retention of foreign bodies in the pharynx, and of diphtheritic or syphilitic pharyngitis; and children with a scrofulous, tubercular, or rachitic taint are more likely to be affected than those not so afflicted.

Symptoms.-The initial symptoms consist of stiffness and pain of the neck and difficulty in swallowing, these gradually increasing in severity, and they may or may not be accompanied by fever. As the disease advances the pain and difficulty in swallowing increase; there may be obstruction to the breathing or paroxysmal cough, a dulled nasal tone of voice, and immobility of the lower jaw. These symptoms may increase until swallowing and speech become impossible, and breathing almost so. On examination, the cavity of the pharynx is found to be diminished by the presence of a tense, round swelling, which on touch is found to tluctuate. If the abscess be due to caries of the vertebra, the symptoms of that condition will soon become apparent by pain, the characteristic prominence, and the occurrence of paralysis. 
The course of an idiopathic abscess has a more acute character than that of one of secondary origin, and it will discharge itself under favorable circumstances, but should this not occur spontaneously, it must be opened by a bistoury guarded to within a short distance of the point by a strip of plaster. Occasionally it has happened that the pus on being discharged by spontaneous rupture of the abscess has produced fatal suffocation by passage into the larynx; in other cases fatal results have also been due to the abscess opening into the spinal cavity, and sometimes the pus has been found to have penetrated into the mediastinum or pleural cavity.

Prognosis.-This is more favorable in the idiopathic abscess than in the secondary form, the latter almost invariably proving fatal.

\section{Parotitis, cynanche parotidea, mumps}

This disease may be idiopathic, and in that form is often epidemic, or it may be secondary or metastatic, in the course of typhoid, scarlet fever, measles, smallpox, diphtheria, \&c.

The inflammation is generally acute, the exudation having little tendency to pass into pus, and being partly interstitial, that is, affecting the acini of the gland, and partly affecting the connective-tissue surrounding the gland (peri-parotitis), this being the case especially in certain instances.

Symptoms and course.-The initial symptoms are the same in this disease as in others of inflammatory origin: shivering or rigors followed by fever, depression, headache, vomiting, and delirium, or, in very young children, even convulsions. After these there gradually appears a painfulness and swelling in the neighbourhood of the cheeks and ears, most pronounced over the parotid gland. This graduaily increases in size, and gives the face a curiously awkward look; the mouth cannot be opened without great pain and, finally, cannot be opened at all; speech becomes very unintelligible, and swallowing very much impeded.

Orchitis is not unfrequently seen either concurrently or immediately following mumps in boys. The parotitis may he unilateral, most frequently affecting the left gland, or may be 
double, the affection of the second gland usually following that of the first in a few days.

The course of the idiopathic and epidemic form of parotitis usually lasts from eight to fourteen days, and almost always results in recovery; whilst the secondary variety generally ends in suppurative destruction of the gland, the discharge being evacuated by the auditory meatus. When the acute inflammation passes into the chronic form, the gland becomes permanently enlarged and indurated. Occasionally cystic and fibroid tumours, and the interstitial deposit of fat (Adiposis parotidea), are met with in this gland; but malignant degeneration of it is very rare in children. The pressure of the enlarged gland on the branches of the facial, or their destruction in the course of suppuration in it, may bring about paralysis of the facial muscles, either temporary or permanent. Angina tonsillaris or pharyngitis are met with as complications or sequelæ of parotitis.

Mumps has been considered as one of the zymotic class of diseases from certain resemblances it has to the well-established instances of that type, in that it seems to have a definite period of incubation, a very distinct course, that one attack seems to protect the subject from the disease for the future, and that there seems to be some grounds for regarding it as contagious when epidemic, though this has not as yet been fully established.

Treatment.-The diet must be carefully regulated, and the patient kept quiet and free from excitement. Local applications of oil and a sheet of wadding secured round the chin make the patient more comfortable, and the use of ointments of a deobstruent and sedative character, such as the mercurial ointment with chloroform or opium, or an ointment containing digitalis, iodide of potassium, and extract of opium, may be of use in mitigating the pain. When suppuration occurs poultices are the best application, and the abscess should be opened as soon as possible. The feverish condition, and any general symptoms, must receive the treatment appropriate to them. 


\section{b. Diseases of the CEsophagus}

Diseases of the œsophagus are rare amongst children, and are not easily diagnosed. They may be congenital or acquired, of the former the most common being the congenital œsophageal fistula. This may be either uni- or bi-lateral, and is generally found as a narrow canal, permitting, only the passage of a very fine probe, which opens on the side of the neck about half or three quarters of an inch above and behind the sternoclavicular joint. It may end as a blind cul-de-sac, or open into the pharynx, or somewhat lower down into the œsophagus. In chewing and swallowing there issues from it a viscid mucous secretion, sometimes purulent, and an attack of coughing may force air through with an audible noise. It is due to the nonclosure of the second or third branchial arch at an early period of fotal life, is often hereditary in certain families, and is almost always incurable.

Less common malformations of the œsophagus are found as ending of the tube in a cul-de-sac near the heart, opening of it into the trachea, defective formation or complete absence of the tube, diminution of its thickness and calibre, its division up the middle into two tubes which open into one tube above and below, \&c. Almost any malformation of the œsophagus produces early death of the infant.

The acquired diseases of the œsophagus which affect its mucous lining are chiefly those common to other mucous tracts, as hyperæmia, catarrh, inflammation and ulceration.

Hyperæmia may be limited to certain sections only of the mucous membrane, or it may affect the whole tract; and it may vary in its intensity, showing gradations of colour from a slightly increased redness to a deep dark colour. Occasionally slight ecchymoses are visible in the tissue of the affected.membrane, varying in size from a pin's point to a linseed. The hyperæmia is rarely a primary affection, occurring generally in disorders of the digestive functions and in the course of acute zymotic diseases.

Catarrh is generally desquamative, the epithelial layer being thickened and white, the longitudinal folds of the mucous membrane being somewhat exaggerated, and the swollen membrane 
injected and secreting an abundant muco-purulent fluid. Superficial loss of substance, corresponding to the follicles, is sometimes seen along with these appearances.

Inflammation of the œsophagus may be of croupo-diphtheritic, phlegmonous or traumatic origin, in the latter case being generally the result of swallowing some caustic substance or some foreign body, as a needle, fish or other bone. In cases of croupodiphtheritis the exudation is found in isolated patches, or uniformly extending along the tube, and it is also seen in some of the acute exanthemata, especially scarlet fever. I have often seen linear streaks of similar exudation along the longitudinal folds of the osophagus, and in two cases I have seen them even in the stomach. The pustulous eruption of variola may also produce inflammation of the œsophagus. In cases when caustic acids or alkalies have been swallowed, especially the latter, the mucous membrane and subjacent tissue are often destroyed to a considerable depth and by the cicatricial contraction of the resulting ulceration, strictures of the œsophagus are formed, sometimes of very narrow calibre. Gangrene is a rare result of the inflammation.

Symptoms. - When œsophagitis exists to a severe degree the symptoms are sharply marked, and the diagnosis is easy in cases where the disease is the result of the use of caustics or the presence of a foreign body. It is less certain when its origin is croupous or diphtheritic. Pain existing along the tract of the œsophagus, between the shoulders, aggravated by any attempt at swallowing, along with an anxious expression of countenance, and the history of some of the above-mentioned causes, point clearly to inflammation of the œsophagus. Severe cases generally end fatally, whilst those of a milder form generally lead to the establishment of a more or less troublesome stricture.

Treatment.-This must be determined in great measure by the cause of the disease. In the case of the impaction of foreign bodies they must, if possible, be removed, either by extraction or by getting them passed on into the stomach, though neither of these is always an easy matter. In a case when a corrosive fluid has been swallowed, the appropriate antidotes must be administered, mucilaginous or oleaginous drinks, with opiates, to relieve the pain, and small masses of ice given to suck at short intervals to relieve the pain. When the cicatricial con- 
traction comes on it must be counteracted by the careful employment of bougies.

Retro-esophageal abscess has been observed by Rilliet and Barthez in very young children, and it is developed very much like the retro-pharyngeal abscess. The symptoms consist of difficulty of swallowing and breathing, the occurrence of vomiting immediately after food, and severe pain in the region of the œsophagus. Perforation of the œsophagus has been noticed in the course of caries of the vertebra, and in such cases it is possible that a sequestrum may pass into the œsophagus, and through the intestinal tract.

Retro-œsophageal abscesses may result in recovery after perforation of the gullet, or the child may become marastic, and the case end fatally.

Stricture of the osophagus is not common in children as a result of new formations, but from cicatricial contraction it is more common, Kilbe having seen forty-six cases.

Compression of the tube from pleuritic or pericardial effusion, or from enlargement of lymphatic glands, is not uncommon.

Diverticula of the œsophagus, though not common, are met with in children both in the congenital and acquired forms, the former being met with in connection with the congenital fistula in the neck or with a cœecal termination of the œsophagus. The acquired variety may be explained by the union of the œsophagus with a suppurating lymphatic gland, specially apt to occur in the neighbourhood of the bifurcation of the trachea.

These diverticula generally are directed backwards, lying to one side of the vertebral column, and they seldom can be diagnosed during life.

Fungoid aphtha of the mouth and throat may extend into the cesophagus, being found as isolated spots or longitudinal streaks, or, as noticed by Remecker, even forming a continuous tubular cast of the gullet. The symptoms include those of the affection of the mouth and throat, with increased difficulty of swallowing and pain in the region of the gullet. It may be fatal. Aqua calcis and solutions of borax are the best remedies, with occasional emetics to facilitate the removal of the masses of exudation. 


\section{c. Diseases of the Stomach and Intestines}

\section{Dyspepsia}

Dyspepsia may be either the expression of a primary functional disturbance of gastric digestion, or it may be a symptom of many very various pathological conditions.

Amongst its symptoms chiefly may be noticed loss of appetite, abundant formation of gas in the stomach and intestines, painful distension of the abdomen, eructation, vomiting, restless and broken sleep, periodic colicky pains, especially after taking food, which are relieved after the passage of flatus, and a sense of fulness and tenderness in the region of the stomach in children who are old enough to describe their feelings. The stools are generally costive, hard and altered, of a greenishyellow or green colour, of a sour smell, and scattered with curdled, cheese-like masses.

Dyspepsia often results in intestinal catarrh, and fungoid aphtha is a frequent complication.

Causes.-Dyspepsia may be due to any alteration which delays digestion or makes it inefficient. Deficient quantity or quality of the nourishment, too frequent and irregular feeding from the breast, some inappropriate condition of the nurse's milk, or its being too young or too old for the age of the child, the occurrence of menstruation or of another pregnancy during lactation, violent mental emotions, errors of diet, and too great prolongation of suckling, may all contribute to the production of dyspepsia in young children.

A more frequent cause than any of these, perhaps, is the adininistration of artificial food at the time of weaning, or in older children of food which, in quality or quantity, is unsuited for their powers of digestion. A very frequent cause of dyspepsia in young children is a condition of too great acidity of the gastric juice. All diseases accompanied by fever, as well as diseases of the nervous system, produce dyspepsia; but it is rare in children that it is found to be the result of organic alteration of the stomach.

Treatment.-This must be especially directed to the cause of the disease, and to the careful regulation of the diet of the patient. When dyspepsia occurs after weaning, especially 
when the removal from the breast has been sudden and somewhat premature, the symptoms may be relieved in a few hours by the use of a good wet nurse, if the child can be induced to take the breast again. Some children will do this even after they have been weaned for some weeks, whilst others refuse it after only a few days' interval. Dyspepsia, which is the result of overloading of the stomach with indigestible food, especially in infants fed with artificial food, requires a diminution of the quantity and a careful regulation of the quality of what is administered to them. When the gastric acids are too abundant, small doses of some mild alkali, as magnesia, bicarbonate of soda or lime water, is the best treatment. Pepsin, either alone or in combination with small doses of quinine, has not seemed to me to be of any very great use. In more advanced children dyspepsia, the result of errors of diet, will be best and most immediately relieved by an emetic and the subsequent use of mild food. A mild purge, of a sixth or a quarter of a grain of calomel repeated once a day for three or four days, will also be found very useful; and colic, the result of flatus, will be much relieved by a little carbonate of soda and fennel water.

\section{Gastric catarrh}

This disease may be met with in the acute and chronic forms in children, the former being the more frequent. The pathological changes, which are not always in proportion to the symptoms observed during life, consist of an increased injection of the mucous membrane, which may not differ in degree from the colour seen during ordinary digestion, in ecchymosis, swelling and loosening of the membrane, and increase of mucus or of muco-purulent secretion. The mucous membrane is somewhat uneven, owing to the enlargement of the gastric glands, and it is altered in colour, especially when the catarrh is of a chronic character. The cavity of the stomach is usually normal, though it may be increased by frequent distension with gas.

Symptoms.-These include distension of the stomach by gas, increased irritability of the organ, or actual pain on pressure over it, especially after food or drink, vomiting of the food unaltered or in a condition of fermentation and mixed with 
yellow-or greyish-coloured mucus, diminution or complete disappearance of the appetite, frequent eructation, a yellow-coated tongue; and a disagreeable sour smell proceeding from the mouth. The children are dull and listless, suffer more or less from feverish symptoms, and if the disease lasts long, they become emaciated.

The bowels may be either constipated or loose; there is generally considerable thirst, and a craving for cold sour drinks. Amongst the complications the most numerous are acute or chronic follicular ulceration, intestinal catarrh, icterus catarrhalis, and aphthous stomatitis. Stenosis of the pylorus is very rarely met with.

Causes.-These include those spoken of under dyspepsia, also cold, foreign bodies which may be swallowed, as copper coins, lead pellets, \&c., lumbrical worms, certain chemical substances of an irritating kind which may have been administered, as tartar emetic, sulphate of copper, ipecacuanha, \&c. Secondary intestinal catarrh generally follows or accompanies some acute or chronic disease, as one of the acute exanthemata, erysipelas, pneumonia, typhoid, cerebral disease, Bright's disease, tuberculosis, rickets, or syphilis.

The disease occurs at all periods of childhood, though its acute form becomes more rare, and the chronic more common, towards puberty.

Diagnosis. - A mistake between this disease and the initial appearances of tubercular meningitis is one apt to occur, but which may be avoided by an attention to the character of the vomit, and a carcful look-out for cerebral symptoms. Typhoid, during the first few days of its course, also sometimes resembles gastric catarrh, as also does the tympanitis ventriculi, so often seen in girls approaching the period of puberty and which occurs much less frequently in boys. This latter condition is sometimes very obstinate, lasting months or even years.

As a rule gastric catarrh will yield more easily to dietetic than pharmaceutic treatment, and should the former fail, the latter may be carried on very much as in cases of dyspepsia. The various relations of the child, which have been already spoken of, must be examined and carefully inquired into, such as the condition of health of the mother or nurse, the quantity and quality of the milk, or of any substitute which the child may 
be having in addition to or instead of his natural nourishment. Catarrh arising from exposure to cold may be treated by diaphoretics, and if the suffering arise from an attack of indigestion it will be relieved by an emetic. If there be persistent vomiting and an abundant secretion of mucus the tris-nitrate of bismuth, in doses of a quarter to half a grain two or three times a day, or effervescent salines will be found beneficial. When there is much pain or diarrhœa opiates are the best remedies, and, on the contrary, when there is constipation rhubarb is useful.

\section{Croupo-diphtheritic inflammation of the stomach; gastritis croupo-diphtheritica}

This is almost never met with as an idiopathic affection, but occurs most frequently as a complication of scarlet fever, more rarely of measles, smallpox, and typhoid ; or it forms one of the phenomena of epidemic pharyngeal diphtheria ; and sometimes is the result of the administration of tartar emetic and sulphate of copper. The exudation usually occurs in circumscribed, isolated spots, and only rarely is uniformly extended over the mucous surface, in the latter case the exudation being most abundant at the free margin of the folds of the membrane. Necrotic destruction of the mucous membrane is sometimes seen.

Symptoms.-These are not always sufficiently distinct to enable us to diagnose the condition with certainty, unless the obstinate vomiting, which is always present, eject masses of the diphtheritic exudation. 'This, however, may come from the throat or air-passages; but when its presence is accompanied by intense thirst, sometimes quite unconquerable, together with distension of and pain in the stomach, it may almost certainly be regarded as coming from a diphtheritic gastritis. The face is pinched, pale, and of an earthy colour; the pulse small and compressible; the temperature lowered; and the child sometimes restless, sometimes drowsy, or even somnolent. These symptoms may be more or less marked by those of the primary disease.

The treatment can be only symptomatic, consisting chiefly of the administration of ice and effervescent waters, opiates to relieve pain, and cold packing externally. Sometimes warm baths are beneficial. 


\section{Round perforating ulcer of the stomach}

This is a very rare affection in infancy, but it has been met with by Billard, Spiegelberg, Hecker, Gunz, Binz, and others, especially in the newborn. It seems to be due to fatty degeneration of the arteries, and its symptom-complex has been described under the name of melæna neonatorum. This includes frequent vomiting, passage of blood by mouth and stool, great restlessness and evidence of pain, with collapse and a very rapidly fatal course, though in some cases all of these symptoms are absent. The treatment must be chiefly directed to the control of the hæmorrhage by the administration of ice, perchloride of iron, \&c., and the application of cold outside to the gastric region.

\section{Hamorrhagic erosions of the gastric mucous membrane}

These are met with in various diseases due to or accompanied by alteration of the blood and in which the walls of the bloodvessels seem to have a diminished capability of resistance, or when there is some obstruction to the circulation, as embolism or thrombosis. The erosions occur as superficial, roundish or streaky patches of loss of substance of the mucous membrane, with soft, dark red, and bleeding basis, usually limited to the membrane, and occurring either isolated or in considerable numbers.

They are generally the result of some other disease, as one of the acute exanthemata, purpura, tuberculosis, pneumonia, cirrhosis, fatty degeneration of the liver, pylephlebitis, or noma, and are very rarely primary.

The symptoms include vomiting of blood, pyrosis, eructations, loss of appetite, severe thirst, and pain in the stomach, though there may be a complete absence of symptoms. The treatment will be chiefly directed by the disease to which the condition is due, and for the special symptoms the administration of ice, astringents, opiates, and bismuth, will be found useful. 


\section{Follicular and tubercular ulceration of the stomach}

Simple follicular ulceration of the stomach is rarely met with, except in cases of chronic follicular catarrh of the large intestine. It occurs in the form of small ulcers, about the size of a linseed, circular, with smooth bases and swollen edges, seated generally near the fundus, seldom on the posterior wall, and which are not numerous, as I have never seen more than four. They give rise to no symptoms of importance, and, therefore, do not call for treatment.

Tubercular ulceration of the stomach is more common in children than in adults, and is discussed in the chapter on tuberculosis.

\section{Gastromalacia; softening of the stomach}

This is generally, though not always, a post-mortem appearance, due to the self-digestion of the stomach. It is most frequently found in the neighbourhood of the fundus, and the coats of the stomach appear to a greater or less extent softened and gelatinous, and of a greyish, green, or dark brown colour ; and the absence of any sharp line of demarcation of the softening and of all signs of congestion or inflammation, together with the acid reaction of the contents of the stomach, conclusively indicate the post-mortem nature of the change. Nevertheless, it would seem, at least from a few cases in my own experience; that this self-digestion of the stomach may occur during life, or at least that it may be thus begun or a disposition laid for it. The predisposing influences seem to exist sometimes in the stomach, especially in a deficient nutrition of the organ, and sometimes in the central nervous system- $a$ fact indicated by the frequent occurrence of gastromalacia in cases of meningitis, hydrocephalus, and fatty degeneration of the brain.

\section{Intestinal catarrh}

This disease may be divided clinically into three well-defined varieties, though if it be regarded from its causes, course, results, 
or the pathological appearances, there are many grades and forms of it.

(a) Acute gastric catarrh; gastro-enteritis choleriformis ; cholera nostras; cholera infantum

The pathological changes seen in this disease, affecting the stomach, small intestine, and occasionally the ascending colon, consist of injection and swelling of the mucous membrane, and when the course of the case is very rapid, extensive destruction of the epithelial coat. Ecchymoses are also sometimes seen. Acute enlargement of the mesenteric glands and general anæmia of the organs, especially of the brain, which often also gives evidence of fatty degeneration and œdema, hyperæmia of the kidneys, and, in occasional cases, acute parenchymatous nephritis, are amongst the more common of the appearances found concomitant in this disease.

Symptoms and course.-The disease is ushered in by diarrhœa, occurring either simultaneously or soon after one another. The stools are often very frequent, from ten or fifteen to as many as thirty or forty occurring in the twenty-four hours, what passes at first appearing of a mud colour, but it gradually becomes thin, and takes on the characteristic rice-water appearance, and the vomited matter has also this resemblance. There may be little febrile disturbance or even an utter absence of it, the temperature sinks, either gradually or with rapidity, the eyes become sunken, the mucous surfaces cyanotic or waxy, the abdomen becomes retracted, and the cushions of fat doughy to the touch, the tongue becomes dry and cold, the throat unquenchable, and the quantity of urine diminished. As the case progresses the respiration becomes irregular and impeded, there is great restlessness, crying, and whimpering, slight convulsive movement, clouded consciousness, somnolence, and the fontanelles, if not already closed, become drawn in. 'These hydrocephaloid symptoms have their origin in the acute anæmia, fatty degeneration and œdema of the brain, and are certainly not often due to uræmia (Kjelberg).

The course of the disease is generally very acute, its duration varying from forty-eight hours to a few days, the tendency to a favorable issue being indicated by the return of yellowish 
flocculent stools, the cessation of the vomiting, rise of temperature, strong pulse, and the occurrence of quiet sleep. Death may arise either from exhaustion or cerebral complications. Sometimes the disease passes from the acute into the chronic form, and then it may last some weeks. As a rule, in its acute form, it is the immediate result of some error in the diet of the child; sometimes it is due to weaning or to the condition of the weather, as in the height of summer; but occasionally it appears in a secondary relation during the course of the acute exanthenata, broncho-pneumonia, \&c.

Prognosis.-This is always doubtful, though even in its severest forms the disease is not hopeless. The occurrence of collapse of brain symptoms must always be regarded as unfavorable.

Treatment.-This must be early and active, for, perhaps, in no disease is delay more fatal. If the child has been prematurely weaned it must be again placed at the breast, either of the mother or nurse; or if it will no longer suck, the milk drawn from the breast must be given to it by the spoon. If the food had previously consisted of cow's milk, that had better be entirely avoided, and diluted beef tea with rice, barley, or oat gruel substituted. Should the child vomit these also some of the other substitutes for the natural food must be tried, such as have been elsewhere mentioned; but the most useful will often be found to be pounded raw meat mixed with red wine given in small and frequent doses, and for drink either rice water, infusion of saleep, or diluted beer which has been boiled may be given with advantage. If the pulse becomes weak and collapse threatens, stimulants must be given freely, such as wine or strong black coffee with a few drops of rum in it, the latter having often been of signal service in my own experience. Warm baths at a temperature of about $37^{\circ}$ or $38^{\circ}$, and, perhaps, with the addition of some mustard, are often very useful. From drugs we do not get much help, though bismuth, either alone or in combination with Dover's powder, is sometimes useful. Astringents, such as acetate of lead, \&c., are seldom of service, and their administration is a very doubtful practice. In cases of acute anæmia resulting from the disease iron and tonics form the appropriate treatment. 


\section{(b) Acute intestinal catarrh}

The characteristic morbid changes consist of streaky or punctiform injection of the swollen and loosened mucous membrane, the epithelial cells are increased in size, softened, and in greater part thrown off, and the Peyerian patches, as well as the solitary glands, are swollen, at first generally injected, and of a dark red colour, but afterwards appear dark grey or light coloured. Serous infiltration of the submucous areolar tissue is often found in the descending colon and rectum. The intestinal contents vary in appearance in different cases, being sometimes thin and very fluid, or even watery, with mucous masses and fibrinous flocculi, in other cases of a greenish, yellowish-brown or clear yellow colour, and in some exceptional instances they consist of thin mucous or muco-purulent fluid. In this disease the mesenteric glands are generally unaltered, though sometimes they are slightly swollen, and of a reddishgrey colour.

Symptoms and course.-Acute intestinal catarrh is not always ushered in alike by diarrhœa, for we often see, especially in children in the second year of life, that the initial symptoms of the disease, such as dyspepsia, colic and restlessness, may exist for a few days, and that during that time there may even be constipation before the occurrence of the diarrhœa. The stools vary very much both in number, consistence, and colour, and though they may be normal at first, as they increase in frequency they will become thinner, of a bright yellow, yellowish or dark green colour, and of a sour smell. In other instances they resemble gruel or cooked white of egg and consist partly of loosely formed masses of freces and partly of a colourless fluid free from odour, and fibrinous mucus in greater or less quantity. In this affection, too, the stools sometimes show streaks of blood. The frequency and the fluidity of the stools seem to have a relation to the extent of the catarrh, and the sour smell and acid reaction are most pronounced when the stools are of a green colour. Meteorism is not always present, though it is generally noticed when there is severe serous diarrhœa in children under two years of age. The occurrence of pain is generally paroxysmal, coming on shortly before the passage 
of a stool, or soon after the taking of food. When the course of the disease is rapid, and collapse occurs early, there is usually no evidence of pain; the appetite disappears, and the thirst becomes very distressing. Vomiting is not regularly present, but dryness of the mouth and lips, a coated tongue, and aphthous condition of the mouth and throat are very constant occurrences. Winen the diarrhœa is frequent, and the stools very acid, intertrigo and excoriations of the buttocks and genitals are apt to occur, and prolapse of the rectum is sometimes met with. The pyrexial symptoins are not usually severe when present, and there is generally a complete absence of subjective symptoms.

A favorable issue is more likely in proportion as the age of the child is advanced, very young children, especially sucklings, having a high rate of mortality from this disease. The passage of the acute into the chronic disease is often experienced, especially when there is some predisposiug cause present which is not removable.

Causes.-This disease seems to affect the sexes alike, to occur most frequently during the first two years of life, and to exist both as a primary and a secondary affection. The causes are to be found in most cases in some quantitative or qualitative error in the nutrition of the child, and have already been referred to under the heads of dyspepsia and gastric catarrh. Besides these, there can be no doubt that such conditions as exposure to cold, damp feet, teething, worms, \&c., have a more or less direct influence in the production of this disease. As a complication or secondary disease, it is met with in typhoid, diseases of the lungs, the acute exanthemata, Bright's disease, rickets, cases of burning, disease of the heart, fatty and amyloid degeneration of the liver, carious disease of the joints, and chronic diseases of the skin.

Under no conditions should it be regarded as a trivial affection, as is too often the case in teething children, for it may give rise to serious after results, as emaciation, anæmia, serous effusion into the brain and marantic thrombosis.

Treatmient.-No treatment will be satisfactory. unless it can include the removal of the exciting cause of the disease, and in each case will be found special demands in this direction. The method of the child's nourishment, the habits of the mother or 
nurse, the residence of the child and the previous history of its growth, are all deserving of the closest attention. The most useful drugs are opiates, either in the form of the syrup of poppy, small doses of tincture of opium, or Dover's powder in doses of a quarter to half a grain in combination with tannin or bismuth, or some astringent, as decoction of logwood or tincture of catechu. When the stools are very acid some antacid should be given, as carbonate of magnesia, lime water, sc. For drink the child should have some demulcent, as decoction of saleep, rice water, oat gruel or milk of almonds, but no water and no cow's milk. Warm baths and clysters containing small doses of opium, are often of great service.

(c) Chronic intestinal catarrh and follicular ulceration; catarrhus intestinalis chronicus; enteritis follicularis; tabes meseraica.

This variety occurs usually in children under two years of age, and its post-mortem appearances vary with its seat, its duration and its cause. The walls of the small intestines are anæmic, unusually white, atrophied sometimes to an extent which makes them transparent and very easily torn; the villi disappear or are very much diminished in size, and are affected by fatty or amyloid degeneration. In the large intestines the walls are of a dirty red or greyish colour, the mucous layer thickened, and the folds well marked. In the early stage the follicles are swollen and surrounded by a hyperæmic circlet, which afterwards becomes of a slatey-grey, and they may remain permanently enlarged, and be seen as little knots the size of a hempor linseed, whitish or greyish-yellow in colour, or they may atrophy and leave little hollows behind. The solitary glands, on the contrary, ulcerate and form little separate ulcers which here and there become confluent; or they may present the form of small abscesses in the mucous membrane. Cheesy degeneration of the follicles, with the subsequent formation of ulcers (the scrofulous ulceration of the intestines of Niemeyer), has also been observed. The mesenteric glands are always implicated, being increased in size, and often affected by cheesy degeneration. 
Amongst the various pathological conditions met with in this disease may be mentioned dysentery, hæmorrhage from the intestinal mucous membrane, blenorrhagic discharge from the rectum, enlargement of the spleen, liver, and kidneys, with fatty or amyloid degeneration of the latter two, general anæmia, and thrombosis of the great veins, especially of the cerebral sinuses.

Symptoms and course.-Usually the most important symptom, both in the acute and chronic forms of this disease, is diarrhœa ; but it is neither so frequent nor severe in the latter as in the acute variety. The stools vary in colour from yellowish-green to grey or brown, sometimes are very watery, and at others pultaceous, mucous, and lumpy, and as the disease advances they become extremely offensive; several loose stools are usually passed at short intervals and then the bowels remain quiet for a few hours, or a normal evacuation may be made and the passage is usually preceded by pain and gurgling in the abdomen. After the disease has advanced to the condition of ulceration (enteritis folliculosa), the stools become muco-purulent and marked with streaks of blood, and the abdomen becomes very painful, especially over the region of the descending colon. Food may be often noticed in the stools, either in a half-digested form or wholly unaltered, indicating the condition known as lientery; and a very distressing symptom is sometimes met with in the form of extreme gaseous distension of the intestines. In some cases the enlarged mesenteric glands may be felt as knots under the abdominal walls, and the lax abdominal skin may be lifted up in folds.

The appearance of a child suffering from this disease is characterised by general emaciation, sunken eyes, a look of suffering about the face, thin dry skin destitute of fat and hanging in folds, the parietal bones of the skull overlapping others, as the occipital, from the atrophy of the brain, and the general growth and development is retarded. The muscles are thin and flabby and the bones small and slender, the skin and mucous surfaces blanched, and, as the disease advances, general anasarca and ascites usually set in; stomatitis is often seen in the course of the disease.

Prolapsus of the rectum and incontinence from paralysis of the sphincter, intertrigo of the nates and genitals, with excoria- 
tions and diphtheritic exudation, gangrenous ulceration, follicular dermatitis, dysentery and hydrocephalus, are some of the many complications to which patients suffering from this disease are subject.

The appetite is only sometimes impaired, and that transiently, for the child often continues to take an amount of nourishment quite out of harmony with the daily increasing emaciation. The thirst is not usually so distressing a symptom as in acute intestinal catarrh, and a favorable result in recovery is not usually met with. In considering the causes of this disease those must be referred to which have been enumerated under acute intestinal catarrh; but the diarrhœa, so often seen in teething children and in those which have been weaned, and also the chronic intestinal eatarrh of children reared on artificial food, must be regarded as having special prominence as its predisposing causes.

Treatment.-The obstinate and asthenic character of this disease necessitates a frequent change both in its dietetic and remedial treatment. Of the former enough has been already said elsewhere, but the use of pounded raw meat mixed with wine may be again recommended. Drinks should be given very sparingly, and should be chiefly of a stimulant kind; but it will often be found that if no drinks be given at all more benefit will be derived than by any kind of medication. Of drugs, the most useful will be found opiates and astringents, and of the latter may be especially mentioned bismuth, tannin, catechu, and logwood; but it will constantly be found that they require frequent changing. Small injections of starch with opium or acetate of lead are often useful, especially if there be ulceration of the lower bowel. When there is incontinence of frecs cold hip baths or injections of cold water and the internal administration of nux vomica will be found beneficial.

\section{Dysentery}

This occurs amongst children sporadically or in endemic or even epidemic extent, and it may be idiopathic or secondary to some other affection.

Anatomy.-This consists of an inflammation of the colon and 
rectum, with exudation of a pseudo-membranous or diphtheritic character, of various extent in proportion to the severity of the case, more or less adherent to the mucous membrane, and, when removed, leaving exposed ulcers of varying depth, irregular in outline, and of varying extent and colour. The mucous membrane is swollen, red, softened, or even loosened by diffuse suppuration. The solitary glands are also found swollen and ulcerated, and the corresponding peritoneal surface is found injected. General anæmia is a very common sequela of the disease, and ulcerative perforation or cicatricial contraction of the gut are met with as more exceptional results.

Symptoms and course.-When the disease is primary its course is generally rapid and acute, but when due to gastric disturbances it has a more chronic form. It is usually ushered in by nausea and vomiting, intense fever and acute abdominal pain, and its most prominent symptoms are the characteristic frequency and appearance of the stools. These are small in quantity, but very numerous, from eight or twelve to as many as forty or fifty occurring in twenty-four hours; in appearance they are at first shiny, with nodules and flocculi in them, but they soon become tinged with blood either in streaks or in clots, or the stool may be uniformly reddened by it. The passage of fæces is accompanied by straining so much that this soon becomes the most prominent and distressing symptom, and it continues without the passage of any fæces. In the evacuations, which at first are fæcal, but later are free from smell, yet again after the formation of ulcers become extremely offensive, are to be found mucus, pus and blood-corpuscles, epithelium, remains of food, vibrios and triple phosphates. The urine sometimes contains albumen.

Among the general symptoms may be included restlessness and broken sleep, due to the frequent pain, anxious expression of face, brownish-white tongue, distressing thirst, great muscular weakness, whimpering, convulsions, soporific decubitus, and in older children delirium, icteric colouring of the skin, and prolapse of the rectum.

If the disease passes off and recovery results, as generally occurs within fourteen days, the tenesmus disappears, the evacuations become less frequent, the yellow colour returns to them, and the blood disappears, and the patient recovers his 
appetite and sleep. Death sometimes occurs with great rapidity when the severe symptoms do not yield to treatment. Relapses are very frequent, and the tendency to them may exist for a long time after the original attack.

Dysentery in children, when primary, is gencrally due to bad, insufficient or unsuitable food, exposure to cold and wet. In the form of diphtheritic inflammation of the colon and rectum it occurs in the course of chronic intestinal catarrh, tubercular disease of the intestine, typhoid, scarlet fever, measles, variola, and cholera; also in marastic children, and in those-who have had prolonged residence in hospital.

Certain observations would seem to show that the disease is contagious, but this has not been quite established.

Treatment.-If the djsease be epidemic in a neighbourhood, any appearance of diarrhœa in a child demands care, and the child should be kept in bed and the diet carefully attended to. In the cases of sucklings no other nourishment than the mother's milk should be given, but in older children such drinks as decoction of saleep, rice water, or emulsion of almonds will be found beneficial.

The medication of the early stage should consist of the cautious administration of some laxative, as castor oil, guarded with a small dose of opium. After this astringents and opiates are to be used, and for the relief of the tenesmus the latter, either in the form of clysters or suppository. When the stools have been purulent $I$ have seen much benefit from clysters containing chlorate of potash. Warm poultices over the abdomen or warm baths relieve the abdominal pain; and so sometimes do cold applications, such as ice-bags, continued for two or three hours. When collapse is threatened stimulants, as wine, brandy, musk, and camphor, must be given freely.

\section{0.-Constipation, Intestinal obstruction, Koprostasis}

This is a condition not very usual in young children, especially when they are reared on artificial food. 'The evacuations occur only once in twenty-four hours, or, indeed, even only once in two or three days, accompanied by great straining, or they may not occur until some kind of assistance has been given, the fæces being hard and dry, and of a light colour, 
somewhat resembling those passed by dogs. The abdomen may be either distended by gas or soft and flattened, and hard masses may be felt in the course of the transverse and descending colon.

The general symptoms consist of loss of appetite, colicky pain in the abdomen, restlessness and disturbed sleep, headache, occasionally delirium and, in infants, convulsions. There is great pain in the rectum and sometimes the gut is prolapsed, with passage of urine and blood; and there is frequently romiting and symptoms of fever. All these may disappear entirely after the occurrence of a few evacuations. When the constipation is very obstinate, the motions, though passed daily, sometimes consist of thin worm-like masses, whilst the more bulky and hard fæces are reiained. The causes of this condition may be very various, but amongst others may be mentioned an insufficient quantity or too viscid quality of the intestinal mucus, a too abundant supply of milk rich in casein, or of starchy food, insufficient drink, and the use of astringent remedies, as lead, opium, \&c. It may also be the result of such causes as diminution of the peristaltic movements of the intestines, paralysis of the muscular coat during the course of some cerebral or nervous disease, or of some mechanical hindrance, as hernia, intussusception, peritoneal adhesions, a number of lumbrici matted together, foreign bodies such as fruit kernels, or, more rarely, from the presence of a stricture in the intestine produced by some new adventitious growth or cicatricial contraction.

Treatment.-Generally this must consist in rectifying any error of diet that may be discovered, the administration of demulcent drinks and fruit either cooked or raw, whilst the costive habit must be overcome by the administration of mild laxatives, such as rhubarb, or, better still, of clysters of soapand-water or cold water only. If there be any hard fæcal masses lodging in the rectum which are not affected by the injections, they must be broken down by the finger, the handle of a spoon or by some other suitable instrument. In cases of habitual constipation occurring in more advanced children, I have seen considerable benefit derived from the methodic employment of gymnastics, swimming, and also of cold-packing on the abdomen. 


\section{Stricture and Occlusion of the Intestinal Canal}

These are not common in children, and when met with are nearly always congenital, the latter occurring most frequently as atresia ani. Intra-uterine peritonitis and ulceration of the mucous coat of the intestines have also been noticed as causes of these conditions. They may also be occasionally met with arising out of some such process occurring after birth, or from the presence of some foreign body. I have seen a case of alveolar cancer of the large intestine in a boy nine years old.

Symptoms and course.-Congenital stenosis is marked by the passage of very little or no meconium, paroxysmal pain, swelling of the abdomen, and the onset of sickness, which lasts until death, unless relief can be given, and the symptoms of any acquired constriction are very much the same.

The treatment of any obstruction must be regulated by the cause, though in most cases it can only be surgical, as in the case of atresia, when, if the gut cannot be reached from the perinæum, it may be opened in the left loin or the small intestines may be opened in the groin. In other cases of obstruction strong purgatives, metallic quicksilver, and the inflation of the intestine from below, have been recommended.

\section{Invagination, or Intussusception}

Intestinal invagination is met with in two forms in children : the first, occurring only in the agony, is of no importance, and is easily undone. Instances of this varying in length from one to four inches are constantly found in post-mortem examinations of children, especially of those who have died of brain disease or of an intestinal catarrh. The second variety is marked by serious symptoms, and may occur in children of any age. In most instances they are descending, occurring almost solely in the great intestine, and usually start from the ilio-cœcal valve. The cause of their occurrence is not very clear, but it most probably consists in the disproportionate width and mobility of the two portions of the tube connected at the valve; and there can be little doubt that the immediate origin of the invagination 
may be some violent blow or pressure on the abdomen, or constipation, or long continued diarrhœa. Anatomically it is characterised by the invaginated portion and its mesentery being swollen and of a dark red colour, and the part adjacent showing evidences of the inflammatory process, in adhesions and the exudation of greyish-yellow lymph. The resulting symptoms resemble those already detailed in constriction or obstruction of the intestinal tract, and they are more or less marked in pro. portion as the invagination is complete and extensive. The matters vomited consist of the food taken, mixed with bile, mucus, and, as the case advances, fæcal matter. The abdominal pain is paroxysmal, and there may be constipation or diarrhœa, the stools being tinged with blood. The face is anxious, emaciation rapid, the pulse thread-like and small, the temperature of the skin low, and the abdomen distended. Sometimes the invagination can be felt as a cylindrical, hard, smooth, and movable tumour in the region of the ascending or transverse colon; in this case it being possible to mistake it for a fæcal accumulation, though it may be possible to make the diagnosis certain by detecting the invagination by the finger, or by its protrusion from the anus, as sometimes happens. As a rule cases of this displacement end fatally, though exceptional instances have been recorded where the invaginated intestine has sloughed and been passed, recovery resulting. This occurred in a boy four years old, under my own notice, when a knuckle of the large intestine, about three inches long, was passed after a ten days' illness.

Treatment.-Purgatives must be avoided, absolute rest must be enjoined, and diet of an easily digested and strengthening kind must be given. It is advisable to push opium to narcosis. When the invagination has been ascertained to exist in the large intestine, attempts have been made to replace it. Gastrotomy may be entertained as a last resource.

\section{Prolapsus ani}

The most common form of this is an extension of the mucous membrane covering the sphincters and lower part of the gut, appearing as a roundish tumour of a dark red colour, glistening, 
bleeding on slight touch, and having an opening in the centre. Another and more rare variety consists of a true invagination, as instanced in a case under my own care, of a boy three years old, where the upper part of the rectum and a portion of the descending colon, in all more than twelve inches long, protruded through the anus. When such protrusions have existed for some time they may be attacked by croupo-diphtheritic inflammation, ulceration, or even gangrene of the mucous membrane. Cases of invagination are sometimes fatal from purulent peritonitis.

Treatment.-This must include the removal of any exciting cause, such as diarrhoa or constipation, and the replacement of the prolapse. The child ought not to be allowed, if possible, to strain much, and for this purpose it is well to have the bowels mored while the patient is lying, or to place the utensil on a chair or stool so that the child's feet cannot reach the ground. Injections of cold or iced water are useful, and in obstinate cases cauterisation of the protrusion with nitrate of silver or with the hot iron may be tried. Guersant recommends punctiform cauterisation round the anus, and excision of some of the protruding mucous membrane has also been performed.

Invagination of the colon and rectum is a rare but dangerous condition, and is to be remedied by the replacement of the invagination by the finger or some appropriate instrument. Its retention may sometimes be secured by a mechanical appliance.

\section{Polypus of the Rectum}

This is a very rare affection, especially in young children. When met with the polypus is generally close to the anus, seldom high up, of the size of a hazel-nut or cherry, readily bleeding, generally protruding at stool especially when pedunculated, and giving rise to colicky pain and ultimate anæmia from loss of blood.

Treatment.-Spontaneous separation by elongation and final tearing of the pedicle sometimes occurs. Removal of the growth is generally necessary, and this may be done either by the scissors or ligature, the latter being preferable, to obviate the risk of hæmorrhage. 


\section{Inguinal hernia}

Inguinal hernia is of common occurrence in children, more so in boys than in girls, and is divided into the varieties of external and internal, both of which may be congenital or acquired. In the congenital hernia the protruded intestine may be in immediate contact with the testicle, or indeed may completely surround the gland (hernia congenita testicularis); or the processus vaginalis of the peritoneum may be closed above the testicle, and only the inguinal portion remain open (hernia congenita funicularis). In girls the hernial pouch occupies the labium majus. Occasionally the hernial sac is found to be double. Congenital hernia is seldom present at birth, but is produced soon after as the result of crying, coughing, and straining at stool.

The acquired hernia in children does not differ in any way from that seen in adults.

The contents of the sacs consist nearly always of small intestine, though occasionally omentum and large intestine are also found ; the vermiform appendix and even an ovary, in some rare cases, have been met with in hernial sacs. The rupture is more commonly single than double.

Symptoms.-These are, as a rule, entirely local, the children, in the majority of cases, suffering but little from the rupture, though in some cases colicky pains seem to be induced. Incarceration of the contents of the sac is not common, for I have seen it only three times out of many hundreds of cases. Occasionally strangulation of a hernia is seen in a child, and is characterised as it is in the adult by hardness and distension of the hernial sac, fixidity of the abdominal walls, eructation and vomiting of the intestinal contents and fæcal matter, constipation, and a peculiar distressed expression of the face.

It nust be remembered that in inguinal hernia it is necessary to take care to avoid mistaking it for a hydrocele, a cyst of the cord, an undescended testicle, or, in girls, a protruded ovary. Occasionally, one or other of these conditions may be found complicating an intestinal protrusion, and to make the diagnosis certain very great care must be taken.

Treatment.-Though it cannot be denied that hernia is often 
cured spontaneously in infants, without any surgical interference at all, and that the trouble of keeping the child's tender skin from being injured by the pressure of the truss is very great, yet I deem it always advisable to fit a child suffering from rupture with a suitable apparatus. Besides this, care must be taken to diet the child so that it has at least one daily evacuation, and to remove all exciting causes of crying and coughing.

The hernia should always be replaced before the truss is applied. In case of strangulation an operation for its relief must be performed. In cases where an undescended testicle complicates a hernia, Guersant and Marjolin advise that both rupture and the gland be replaced and kept up by a truss.

\section{Animal Parasites. Worms, Helminthiasis}

Intestinal worms may exist in considerable number, and for a long time, in a child, without attracting notice by any symptoms, though they may reveal themselves by indications which may be local, general, or reflex. An interesting fact has come under my notice, that in some years intestinal parasites seem much more common than in others, and it would seem of them, as of the acari of scabies, that they thrive best from some conditions which exist outside man.

The most important intestinal parasites of children are the following:-

\section{a. The Round Worm, Ascaris lumbricoides}

This belongs to the order of Nematodes, and is the most frequent intestinal parasite of children. 'The length of the male is twenty-five centimetres, that of the female forty, the body round and of a yellowish red colour, at the anterior extremity of which there are three thick lips placed together, constituting a button-like head, and the edges of the lips are dentate. The posterior extremity of the male is strongly curved, the sexual canal being placed at the posterior part of the abdominal surface, that of the female in the middle. The production of ova is very abundant, these measuring, according to Leuckhart, 05 to $.065 \mathrm{~mm}$. in the larger and $.043 \mathrm{~mm}$. in the shorter diameter, 
and are usually surrounded by a yellowish albuminous envelope. The parasite inhabits the small intestines, and may be single or occurring in great numbers, as many as a hundred having been seen in one case. I have often seen an increased redness and swelling of the mucous membrane and an increase of the secretion of mucus, mark the seat of a colony of lumbrici.

The statement that lumbrici perforate the wall of the intestines has not been substantiated, but they certainly wander about and find their. way through the bile ducts into the substance of the liver, also into the stomach, œsophagus, and cavity of the nostrils, the larynx, trachea, and bronchi. Under the influence of certain acute diseases, and of certain kinds of food and drugs they are readily passed by the rectum.

Symptoms. - The appetite is generally disturbed, being sometimes diminished, sometimes increased and perverted with a special prediliction for farinaceous food, bread, cakes, and sweets; there is sometimes constipation, sometimes diarrhœa; the abdomen is distended and affected by griping colicky pains, especially in the umbilical region; the face becomes bleached, grey, or earthy in colour, and the eyes dim and watery; the patient picks the nose and grinds the teeth, during which sleep is disturbed and restless; and there is frequent vomiting, with a disagreeable and sour smell of the breath. If the worms migrate to the rectum they create much uneasiness there, indicated by the child scratching the arms and genitals; and if they get into the bile ducts they produce jaundice (hepatitis verminosa). I saw very severe jaundice, lasting for some weeks, in one case, where, on section; we found a lumbrical worm in the bile duct. Impaction of round worms in the larynx and bronchi has given rise to fatal pneumonia, and a number of worms collected into a firm ball has been recorded as a cause of intestinal obstruction. It has often been asserted that the presence of intestinal worms has given rise to or has sustained the existence of eclamptic convulsions, chorea, and epilepsy; but $I$ have met with no distinct evidence of this, and cannot but think more care ought to be taken in investigating the cases on which such assertions are based.

Diagnosis.-This is easily established when the worms are passed, or when their ova are found in the stools by microscopic examination. 
Treatment.-In cases where their presence is suspected the administration daily of some sour white wine will soon display them if they exist, but the surest remedy is Santonine in doses of one or two grains given two or three times a day, in combination with calomel or quinine. An elegant preparation of this drug may be given in comfits or worm-cakes; but in this way the dose is somewhat uncertain. Flowers of Cina, in doses of ten or twenty grains boiled in milk, and infusion of garlic administered by injection are also very useful. When santonine is given chromatopsia sometimes occurs, but it is quite ransient and free from ill effects.

\section{b. Thread worms; Oxyuris vermicularis}

These parasites are white in colour and round, the male having a length of four millimetres and the female about ten, the oral extremity being somewhat thickened and bladder-like, and the tail end in the female is shortened and shaped like an awl. The ova are very numerous, Leuckardt having reckoned that a pregnant female has in her from ten to twelve thousand, and they are oval in shape, measuring about $.052 \mathrm{~mm}$. in length, and about half that in breadth. They usually inhabit the rectum, though they may be found high up in the large intestine. The lumbrial worns and the trichocephalus are often found in association with them.

Symptoms.-These are chiefly local, and consist of a very persistent and troublesome itching of the anus, which gives rise to much nervous irritation and restlessness, especially towards evening and during the first sleep. Sometimes the worms migrate into the vagina, and there excite great irritation, catarrhal vulvitis, leucorrhœa, and menstruation. In the male they cause erection and sometimes balanitis, also pain on micturition and defecation. If allowed to exist for a long time, they may interfere with the nutrition of the child. 'They occur very rarely in sucklings, being met with generally in children somewhat advanced.

For the treatment cold hip baths, injections of cold water or infusion of garlic, and the insertion of suppositories of styrax or blue ointment, are the most beneficial. In very obstinate cases the internal use of santonine and calomel may also be employed. 


\section{c. Tapeworm; Tania}

The varieties of this class met with in children are $T$. solium, T. mediocanellata and Bothriocephalus latus.

The first may be seen in great lengths, sometimes from fifteen to thirty feet, consisting of numerous joints, like melon seeds. The head is small, and is provided with four suckers, a rostrum and corona of hooklets. The perfect joints have at the margin perforated prominences marking the genital orifice, these appearing alternately on the right and left sides. The $T$. solum originates in the measles of swine.

The T. mediocanellata differs from the $T$. solium in that its head has no corona of hooklets, though provided with four suckers. Its posterior joints are exceptionally broad and thick, and are spontaneously separated. The genital canals are lateral and alternate irregularly. It is developed from measly beef.

In the Bothriocephalus latus the joints are as broad as long, and lie over one another like the tiles of a roof. The head has no sucking-cups nor hooks, but only two cleft-like suckers. The sexual canals are not lateral, but placed in the middle of the joints.

In a few cases $T$. elliptica has been found in children.

Symptoms.-Very frequently the presence of these worms is never suspected, from an utter absence of any symptoms, until the passage of joints has been noticed. In other cases they give rise to such symptoms as loss of appetite or inordinate hunger, frequent transient colicky pain round the umbilicus, feeling of pressure and burning over the abdomen, itching at the nose and anus, squeamishness and tendency to vomit, especially in the morning when the stomach is empty, headache, fits of loss of power, restless sleep, grinding of the teeth, and nervous attacks resembling chorea, eclampsia, or epilepsy.

I have seen tubercular meningitis and chronic hydrocephalus occur in patients affected with tænia, and it requires much caution to discriminate the true cerebral symptoms of such cases from the symptoms due to the helminthiasis.

The most important sign for the diagnosis of tape-worm is, of course, the passage of some joints. 
Treatment.-The following anthelmintics have been recommended for the treatment of tænia, but there are many cases where the parasite is so persistent that all remedies fail.

Kousso, the flowers of the Brayera anthelmintica, given in doses of one to two drachms in some honey to the patient fasting; also kamala in doses of half a drachm to a drachm. The bark of the pomegranate root has also been recommended, from half an ounce to an ounce being macerated in a pound of water for twelve hours, then boiled down to half a pound and taken in three doses at intervals of half an hour. The extract of Filix mas is very generally of use, and I usually give twenty grains of it in combination with a drachm of kamala. These remedies may be given without hesitation to children, even when very young, and no special preparations are necessary. It is generally advisable to follow them by some active laxative.

\section{(d) Trichocephalus dispar}

This belongs to the class of nematodes, and does not come often under our notice. In length it measures from one and a half to two lines, the anterior part being thin and hair-like, the posterior somewhat thicker and spirally curved in the male. It chiefly inhabits the large intestine and cæcum, and from its size may very readily be overlooked by a careless observer. It is of little importance, either clinically or pathologically, and rarely calls for any therapeutic interference.

\section{Diseases of the Peritoneum}

\section{Peritonitis}

As in adults, this disease may occur in children in the acute or chronic forms; may be general or local, idiopathic or secondary, or metastatic.

Anatomy.-In acute general peritonitis the vessels of the peritoneum or of the subserous tissue are intensely congested, and the membrane is marked by a reddening which is either general or limited to circumscribed spots, by spots of ecchymosis, and 
by thickening and opacity. Its cavity is occupied by serum, which may either be clear or mixed with pus and flakes of fibrin, or the fluid may be completely purulent, and it is found abundantly in the pelvis and between the folds of the mesentery. When acute peritonitis is limited in its extent it gives rise to new formations in the connective tissue, to omental and intestinal adhesions, and occasionally to localised suppuration.

Chronic peritonitis may be local or general; in the former variety giving rise to plastic adhesions, pseudo-membranous exudations, or sacculated abscess, which may be evacuated through the abdominal wall, the intestinal canal, the bladder, \&c. When the affection is general there is an abundant serofibrinous exudation which sometimes appears as a thick uniform membrane; and occasionally this membrane may show evidences of fatty, chalky, or cheesy degeneration. Chronic peritonitis is very often of a tubercular character.

Symptoms and course.-The severity of the symptoms is generally proportionate to the extent of the inflammation, the quality of the exudation, and the acuteness of the course. The more prominent initial symptoms are generally rigor and vomiting of yellowish-green mucus. The local symptom of pain is never absent, and when the peritonitis is general, it extends all over the abdomen. It is generally of a sharp, stinging character, increased on pressure, coughing, deep inspiration, or by vomiting, and it disappears only towards the end of the disease. The febrile symptoms are usually severe, though cases occasionally occur where they are completely absent. Thirst is a very troublesome symptom in many cases, the appetite being completely lost, and in the case of sucklings the breast is refused. The face is expressive of great suffering, and blanched or earthy in colour, and the patient almost invariably lies immovably on the back with the knees drawn up, shrinking from any attempt to move him. The respiration is retarded and superficial, being almost exclusively carried on by the muscles of the thorax. The physical signs include more or less meteorism, dull percussion.note, and evidence by fluctuation of the presence of fluid in the peritoneal cavity, and by friction sound in the upper half of the abdomen of fibrinous effusion. Of the general symptoms the most important are constipation, dry and coated tongue, disagreeable taste in the mouth, hiccup, retention of 
urine when the inflammation involves the region of the bladder, vomiting and nausea, delirium, and, in sucklings and young children, convulsions. When the disease has a very rapid course in sucklings the symptoms may be very slight, or be limited to expression of pain and distension of the abdomen.

If the case is likely to result in recovery the symptoms gradually abate. When the inflammation has been circumscribed recovery sometimes takes place by perforation of the abdominal wall and discharge of the ichoro-purulent fluid which has been contained in the peritoneal abscess, as I have seen in several cases where the opening took place in the neighbourhood of the umbilicus, and in one where it occurred, after perforation of the intestine, in the left groin, and resulted in a permanent fæcal fistula. When the disease takes a chronic course the pain abates or takes on a paroxysmal character; the patient becomes feverish towards evening, suffers from frequent vomiting and from constipation, alternating with diarrhœa, and becomes emaciated, the skin getting dry and tender; and, finally, death occurs from exhaustion or from an acute exacerbation of the disease.

Hiccup is a frequent symptom in acute peritonitis, and retention of urine sometimes occurs from effusion in the neighbourhood of the bladder.

Causes.-Peritonitis may occur during intra-uterine life from syphilis or some septic infection of the mother; or it may be caused during the first week of life by inflammation, suppuration, or gangrene of the navel; by atresia ani, or from purulent infection from a mother suffering from puerperal fever. Later in life it may occur as a secondary affection to some of the acute exanthemata, or to such diseases as nephritis, dysentery, \&c. Idiopathic peritonitis arising from some exposure to cold, or the traumatic form following blows or operations, such as lithotomy, is not often met with among children. Peritonitis from tubercular or typhoid perforation of the intestine in children is equally unusual.

Diagnosis.-The pyrexial nature of the disease in acute cases, the collapse, and, above all, the characteristic pain, permit of little doubt for the diagnosis; but in chronic cases there may be some difficulty in distinguishing it from ascites due to some other lesion, and then a careful inquiry into the history and 
possible exciting causes should be made. Sacular peritoneal abscess may be mistaken for simple abscess of the abdominal wall. In order to arrive at a knowledge of the character of the effusion it is very important to determine whether the peritonitis is idiopathic, secondary, or metastatic.

In chronic peritonitis percussion gives a dull note, and pressure excites pain. There are marked nocturnal exacerbations of the pyrexial symptoms, and the constipation often alternates with diarrhoea ; and these symptoms are the more trustworthy if acute peritonitis has preceded them, or if there are concomitant symptoms of disease, especially of an inflammatory character, in some of the abdominal viscera.

Prognosis.-In all forms of the disease this must be given with caution, for in children it is always a serious affection. Death may be very rapid, occurring within forty-eight hours after the onset of the inflammation, especially in the peritonitis of new-born and suckling children, and in cases of the septic variety.

Acute idiopathic peritonitis may last from eight to twentyone days, whilst the chronic disease may go on for weeks or months with intermittent acute exacerbations. The most favorable prognosis belongs to cases of primary and circumscribed inflammation of the peritoneum with bland exudation.

Treatment.-The most important points of the treatment consist of absolute rest, nourishing diet, and in the early stages the use of antiphlogistic and sedative remedies. The abdomen may be covered by ice or cold water packing, which will often be found to diminish the intensity of the fever and pain. I begin the treatment with a purge of a grain or two of calomel, especially when the bowels have not been opened, to be followed by opiates. The thirst and vomiting will be found to be much assuaged by sucking pieces of ice. For patients who cannot submit to cold applications to the abdomen, warm baths and light poultices over the abdomen are to be recommended, and the baths are better than any other application when any local suppuration is suspected. If the exudation be abundant and the pain abating, inunction of mercurial and iodine ointments may be found useful in favoring absorption. The strength of the patient must be carefully sustained, and any symptoms of exhaustion are to be treated by strong soup and stimulants, as 
wine, \&c., and quinine; the latter drug is especially to be given in the septic variety of peritonitis. When there is any remaining pain, frequent warm baths, opiate clysters and counter-irritation by tincture of iodine, will be found useful.

\section{Tubercular Peritonitis and Tubercle of the Peritoneum}

These conditions are common in children, for of 800 cases of tubercle, I have found its seat to be the peritoneum in ninetytwo.

The pathological appearances are very similar to those already described of tubercle elsewhere, the seat of the exudation being sometimes limited to the peritoneal surface of the intestine, whilst in other cases it is spread uniformly over the whole of the serous membrane. There is generally some hyperæmia with numerous perceptible, greyish tubercles, more or less pigmentous infiltration, adhesions between the coils of intestines, and generally some sero-purulent effusion, which in some cases is mixed with blood. I have twice seen an abscess form and perforate the integuments in the neighbourhood of the umbilicus. The omentum is usually puckered up in the upper part of the abdomen, and occupied by exudation. Cheesy and tubercular alteration of the mesenteric glands and tubercle of other organs are very often found in association with tubercle of the peritoneum.

Symptoms.-The children affected are generally such as have previously exhibited some indications of tubercle or scrofula, the peritoneal affection being only a later phase of the general condition. The most important symptoms are fever and pain in the abdomen increased by pressure, these being rapidly established in acute cases, but more insidiously when the disease occurs in a chronic form. The abdomen is distended, sometimes irregularly, and in the later stage fluctuation may be perceptible, especially at the lower part; constipation may alternate with obstinate diarrhœa. Dysuria and colliquative sweating, with œdema of the lower extremities produced by obstruction to the return of blood or thrombosis of the veins are also met with as general symptoms. In some cases, especially of very young children, tubercular peritonitis is accom- 
panied by such slight symptoms that a diagnosis is scarcely possible. In acute cases its duration is usually only a few days, but when chronic it may last several weeks, months, or even years, its course varying in severity, sometimes apparently coming to a standstill, and again rumning into acute exacerbations.

The causes.-These are the same as in other scrofulous and tubercular affections, children between four and ten years of age being the most frequently affected, though it is not uncommon in sucklings and during the first years of life. Boys seem to be more frequently attacked than girls.

The prognosis must generally be unfavorable, the more so in proportion as the course of the disease is acute and painful, and the more extensive the surface of peritoneum affected. Favorable results are very rare.

Treatment.-In the direction of prophylaxis this must include all the general directions to be given in cases of scrofula and tubercle of other varieties. In the general treatment the syrup of the iodide of iron finds great acceptance, and quinine with an opiate, such as Dover's powder, when there is 'diarrhœa, or cod-liver oil when there is no diarrhœa. Pain is to be relieved by opiates, warm fomentations, and poultices over the abdomen. When there is much ascites present diuretics and diaphoretics may be given, and when it is extreme the peritoneum may be tapped.

\section{Ascites}

The collection of a quantity of clear serum in the cavity of the peritoneum, constituting ascites, is never a primary disease, but always the indication either of general dropsy or of some local affection, and it may coexist with other forms of dropsy or be met with alone.

The most usual causes of ascites are the hydræmia, resulting from acute or chronic parenchymatous nephritis; obstruction to the return of venous blood, resulting from disease of the heart or lungs; disease of the peritoneum; disease of the liver and portal vein; and tumours of the abdomen, especially enlargement of the lymphatic glands.

Symptoms. - When the amount of the effused fluid is small its 
indications are not definite, but when the quantity is moderately large the abdominal walls are arched forwards, and fluctuation becomes distinct. Percussion over the prominent part where the intestines float gives a clear percussion note, which alters in relation to the position of the patient. At the lower and lateral regions of the abdomen, when the patient is on his back, the percussion note is dull. The cutaneous veins are very prominent, there is pain in passing water, and the bowels may be either costive or somewhat relaxed, and the breathing is often impeded from pressure on the diaphragm. The urine is frequently diminished in quantity, of a dark colour, and contains albumen, blood, and fibrinous tube-casts.

Diagnosis. - When the peculiar mobility of the clear percussion is present there is usually no difficulty, and passire ascites may always be distinguished from effusion the result of peritonitis, by the absence of fever and pain on pressure.

The prognosis is generally unfavorable, though this is to be determined by an appreciation of the cause, of the effusion. which, in some cases, may be curable.

'The treatment must also be directed by a consideration of the causes. The symptomatic treatment should consist of the administration of diuretics, diaphoretics and hydragogue purgatives, the first class being better avoided when the ascites is due to acute Bright's disease. I have seen some very unexpected and favorable results from the purgative treatment. When the distension is great and the respiration impeded tapping will always yield relief, and occasionally even effects a cure.

\section{E. Diseases of the Liver}

Diseases of the liver, if we except fatty degeneration, are not so common in childhood as in advanced life. Amongst 40,000 cases of sick children I have found 425 cases of independent diseases of the liver. Of these icterus catarrhalis and neonatorum are the most common, and next to them,in frequency rank fatty and amyloid degeneration of the liver. Cirrhosis, syphilitic and tubercular deposit in the liver, and hepatitis parenchymatosa, 
are uncommon; whilst cancer is almost unknown, and echinococcus is very rare amongst children.

\section{Icterus, jaundice}

Under this head we may speak of the icterus neonatorum and the jaundice of later life as two separate conditions.

The former is not an independent disease, but only the symptom of some affection which may be due to a variety of ætiological factors. Sometimes it is slight and transient, but in other cases it is serious and involves risk to life. In the slightest cases it is a mere excess of the physiological colouring of the skin, occurring especially in premature children during the first few days of their life, the skin and urine remaining unaltered, and the fæces retaining their yellow colour.

In another class of cases the jaundice is due to some catarrhal affection of the bile-ducts arising from errors in the temperature of the baths in which the child is washed, exposure to cold, or from irritation due to the meconium; or it may be mechanical from occlusion of the bile-ducts or compression of their radicles in congestion of the liver. It may also be due to fatty degeneration of the liver in new-born children, and in very rare cases to congenital absence of the cystic or hepatic duct. Finally, it may be due to some blood alteration, and in this case is almost always pyæmic, having its origin in umbilical phlebitis.

From the examination of six hundred and ninety new-born children Kehrer gives the following results :

About two thirds of all children give more or less distinct indications of jaundice within the first week of life, about 6.6 per cent. more of the cases occurring in males than in females, and the affection being on the whole more marked among the former. In the children of primiparæ icterus occurred 4.2 per cent. more frequently than in children of multiparæ, and it was $17 \cdot 7$ per cent. more frequent in premature children than in those born at the full time. It is noteworthy that the discoloration is very frequent over the region of the pelvis, and that the early passage of the meconium, or its retention, does not seem to influence its production.

Kehrer believes that there are three possible methods of the 
production of jaundice in new-born children - a congenital narrowness of the gall-ducts, by which the secretion is hindered from passing freely away; an increased secretion from Hennle's glands; and an insufficient contraction of the ductus choledochus.

The jaundice seen in more advanced children is usually the result of a gastro-duodenal catarrh, arising from some exposure to cold or some error of diet, and it is usually sporadic, though some observers (Rehn) have met with it in epidemic frequency. It has also been found to be due to impaction of lumbrical worms in the gall-ducts, and to compression of the same by hyperplastic and cheesy glands. Koprostasis, hepatitis parenchymatosa, phosphorus poisoning and pylephlebitis (Löschner), may also be mentioned as occasional causes.

Symptoms.-The skin and visible mucous surfaces are of a more or less intense yellow or brownish-yellow colour, and in the former there is often a troublesome itching. The constituents of the bile, especially its acids, are found in the blood and urine, the freces are of a clay colour and devoid of their characteristic odour, the liver is tender or even painful on pressure, the pulse is retarded, there is a disgust at food especially meat, and there is a craving for drinks especially of a sour kind. The sleep of the patient is broken, his movements listless, and when the disease is severe there may be cerebral symptoms, as delirium, mania, convulsions or coma. Sometimes hæmorrhages occur, especially from the navel in newly born children. In the dark-coloured urine the bile acids may be recognised by Pettenkofer's colour-test, sulphuric acid and sugar; but when the jaundice is due to ichoræmia this does not hold good, for the urine is not much discoloured and the faccs are yellow.

Prognosis.-This is always favorable when it can be determined that the case is one due to a simple catarrhal affection; but when there exists any mechanical obstruction or any organic disease of the liver, any opinion given must be cautiously expressed.

Treatment.-Slight cases recover within two or three weeks without interference, and at most require little but occasional warm baths, and, if there be no action of the bowels, some slight purgative, as rhubarb and soda, with attention to the diet of the 
child. Frerichs has recommended citric acid as a useful remedy, and chlorine water; also the alkaline mineral waters, especially that of Karlsbad Mühlburn, given freely. The more severe cases resist all remedies, but some general benefit may be derived from quinine.

\section{Fatty infiltration of the liver}

In this condition the amount of fat in the hepatic cells is much increased, though the cell-walls remain uninjured. On post-mortem examination the liver is found increased in all its measurements, the surface oily and glistening, the margins rounded, the substance doughy to the touch and, on section, of a uniform whitish-yellow colour, interspersed with brownishred spots, and on microscopic examination granules and globules of fat are found to abound everywhere.

Causes.-Fatty infiltration is not always pathological, but is often only physiological and transitory, but in the former case it may be the result of disproportionate and unsuitable food, especially when, as is too often the case amongst poor people, it is too rich in hydro-carbons. It may also accrue from insufficient exercise and from chronic and exhausting disease, as tubercle, scrofula, caries of bone, intestinal catarrh, rickets, syphilis, and many other unknown causes.

Symptoms. - When the amount of infiltration is slight and transitory there may be an entire absence of symptoms; and - even when they do exist, they seldom present the severity met with in adults. The increase in size of the liver may be determined by palpation and percussion, and there may be some tenderness of the organ on touch. Disturbance of the digestive organs from obstruction to the portal circulation is not uncommon. There is rarely any jaundice or ascites, so that in well-marked cases there is usually no difficulty in the diagnosis.

The treatment must depend on the cause, and be in great measure dietetic. 


\section{Fatty degeneration and atrophy}

Fatty degeneration of the liver occurs much more rarely in children than fatty infiltration, and it does not always have a definite character, but may differ in accordance with the cause. The glandular parenchyma is its primary and most usual seat, though in its later stage it affects the whole organ, but in some exceptional cases only parts of the gland seem to be attacked.

During the course of certain acute febrile diseases, especially the acute exanthemata, such as scarlet fever, smallpox, measles, and typhoid, also pyæmia, fatty degeneration of the liver may occur without any well-marked symptom; or it may be the result of some general exhaustive disease of a scrofulous, tubercular, or rickety character. It may also be found as'a part of a general fatty degeneration in new-born children, and in cases of arsenical and phosphorus poisoning.

I have seen fatty degeneration of the liver the result of general parenchymatous hepatitis, with coincident fatty degeneration of the kidneys, heart, and brain, in cases where it seemed to be an independent disease, and inexplicable by our present knowledge of the process. In very severe cases ending fatally I have secn the urine loaded with bile-pigment and the bile-acids, and such symptoms occur as mania, convulsions, tetanic spasm, extravasation of blood under the skin and mucous membranes. The liver was not found softened, but somewhat diminished in size, but neither in it nor in the urine was there any leucin or tyrosin, but numerous fat-globules in both.

The so-called yellow atrophy of the liver, in which there is abundance of both leucin and tyrosin to be found in the liver and urine, is very seldom met with in childhood.

In both of the two last-mentioned diseases the course is almost always disastrous, and any treatment must be purely symptomatic, and with but little hope of a good result.

\section{Lardaceous, waxy, or amyloid degeneration of the liver}

This is much more rarely met with than the fatty alteration of the liver, and it is always characterised by a marked increase 
in size of the organ; in one case, of a boy thirteen years old, it having been found to weigh seven pounds ten ounces. It is always much firmer than normal, the edges are rounded, the colour is greyish-yellow, greyish-red, or light brown, and the cut surface is smooth and glistening and covered by a small amount of viscid bile. On microscopic examination the small blood-vessels and, to a greater or less extent, the glandular acini are found to be occupied by a transparent mass, which, when treated with iodine and sulphuric acid, gives a violet or reddish reaction. The amyloid degeneration is seldom limited to the liver, the spleen, kidneys, intestinal mucous membrane and the lymphatic glands, being more or less implicated.

The disease may exist independently, though it is very generally found in association with cachectic conditions and chronic suppurations. I have seen it in children as early as the fifth week of life, but it is most frequently seen in children after the fifth year, and boys seem more subject to it than girls.

The amyloid change in the liver is always chronic in its course. The abdomen becomes distended and the cutaneous veins prominently marked; the gland can readily be mapped out by palpation and percussion; it is hard to the touch, and the surface is smooth with the edge bluntly rounded. In the course of the disease the patient becomes emaciated and spanæmic; the appetite is much impaired, with a marked loathing against animal food, and the case is closed by general dropsy and indications of kidney complication.

The treatment can only be general and symptomatic unless the cause of the condition can be ascertained and treated. Good nourishment, quinine and iron, country air, warm springs as Marienbad and Karlsbad, are to be recommended; and; when dropsy sets in, diuretics, diaphoretics, and, ultimately, purgatives must be used; but the results are not favorable, the disease being almost always fatal.

\section{Syphilitic inflammation of the liver}

This is very commonly met with in new-born children and sucklings, but it is rare in more advanced children, and it may 
affect the liver generally or only in part. In the former case the liver is markedly larger, its tissue is hard, elastic, and glistens like flint (Gübler), and has an abundant increase of the interstitial connective tissue of the parenchyma. In the partial affection of the liver insulated patches of the hard altered tissue are found seattered through the gland, or the new formation occurs as sharply defined nodules of the size of hazel or walnut, with a firm external layer (syphilitic gummata). Peri-hepatitis, with proliferation of the connective tissue of Glisson's capsule, especially over the affected parts, is also commonly found.

Symptoms. - When the affection is slightly marked a diagnosis is searcely possible, but when severe, the liver will be found enlarged, hard, and by compression of the bile-ducts and of the blood-vessels jaundice will generally be induced. Ecchymosis of the skin and ascites are often also present, and the diagnosis will be completed if indications of syphilis be found on the skin and mucous membranes.

The prognosis must generally be unfavorable, and any treatment must be directed against the cachexia.

\section{Cirrhosis of the liver}

This is a very rare disease amongst children. It consists of a hyperplasia of the interstitial connective tissue, with, in the first stage, increase of size of the organ, followed by diminution. The capsule is generally of a milky opacity, the surface shows numerous slight indentations, and the colour of the glandsubstance is a bright yellow-brown, but sometimes of a bronze colour from infiltration of pigment. The consistence of the gland is firm, its edges obtuse, and a cut surface is brawny and greyish-red, from the numerous trabecula of immature connective tissue.

In such cases I have always found an enlargement of the spleen, and frequently some other condition, as chronic intestinal catarrh, parenchymatous nephritis, fatty degeneration and hypertrophy of the heart, lobular pneumonia, œdema of the lungs, purulent meningitis, and cerebral œdema.

The causes of this disease are not yet known. The cases which have come under my own observation have occurred 
between the sixth and twelfth years, and boys have been more frequently affected than girls in the proportion of three to one. Weber has found it in a new-born child.

The symptoms, prognosis, and treatment, are the same as in adults, and the last is altogether useless.

\section{F. Diseases of the Spleen}

\section{Acute enlargement}

This is met with in typhoid and intermittent fevers, and also generally in acute diseases, such as scarlet fever, smallpox, diphtheria, purpura, pyæmia, \&c.; also in local inflammatory processes, especially in croup, pneumonia, interstitial and parenchymatous hepatitis, erysipelas, \&c.

The enlargement is uniform, never reaches to the size seen in the chronic enlargement, and the capsule of the gland is stretched, wrinkling as the swelling disappears. The tissue is hyperæmic, friable, and pultaceous.

\section{Chronic enlargement}

This may be due either to simple hyperplasia of all the elements or to some additional alteration in texture, the latter being especially frequent in the form of amyloid and lardaceous degeneration. The increase in size is sometimes very considerable, the enlarged spleen occupying as much as half of the abdominal cavity, and presenting a smooth firm surface, anæmic or pigmented, and in the case of amyloid degeneration the cut surface presents a glistening waxy appearance. I have frequently seen fatty liver in conjunction with enlargement of the spleen.

The most important predisposing causes of splenic enlargement are rickets, chronic intestinal catarrh (forty-two out of 100 being due to this disease), tuberculosis, scrofula, caries, disease of the heart, and syphilis. 


\section{Splenitis, inflammation of the spleen}

This disease is very rare, and is never primary, but always secondary or metastatic. The inflammatory centres are usually sharply defined and wedge-shaped, dark red at first in colour, but afterwards of a yellowish-brown or bright yellow, friable, and, in exceptional cases, occupied by small abscesses. They are usually the result of embolic impaction of exudation from the valves of the heart, or of purulent particles in cases of pyæmia.

The symptoms include more or less marked enlargement of the spleen, with tenderness on pressure, and in cases of suppuration, shivering, or rigors.

As in some degree associated with the inflammatory process, we may mention syphilitic new formations in the spleen, occurring as dry, lardaceous-looking nodules, of a whitish-grey colour; lymphoma occurring in leucocythæmia and pseudoleucocythæmia as rounded flat nodules, the size of a pea or cherry-stone, and whitish-grey in colour; and, finally, the much more frequent tubercle of the spleen seen as grey miliary nodules or masses of larger size, occupying the peritoneal surface and scattered through the substance of the organ.

\section{Movable spleen}

I have noticed in a child two years old, suffering from chronic follicular enteritis and broncho-pneumonia, that the spleen was moveable, and could sometimes be felt low down in the left abdominal region between the pubis and umbilicus, and that, if replaced in its usual position, it would not remain long there. Slight pain was induced by its replacement, but otherwise it gave rise to no symptom. 


\section{SIXTH DIVISION}

\section{DISEASES OF THE URINARY AND SEXUAL ORGANS}

\section{Malformations of the Kidneys}

These have only an anatomical interest, producing in life no symptoms calling attention to them, and they come, therefore, very rarely under clinical notice.

'I 'hose which have come under my own observation include absence of one kidney, the remaining organ being very much increased in size, a condition which I have seen of great importance in some diseases, such as parenchymatous nephritis; horse-shoe kidney, when the two glands are usually united in the middle line over the bodies of the vertebræ; and abnormal smallness of one kidney whilst the other is of normal size, the variations of this condition being numerous and of no great importance.

\section{Hyperamia and Anamia of the Kidneys}

Hyperæmia is more frequent than anæmia, and it may affect the whole gland or be partial, and be either arterial or venous. When it is arterial the nodules are injected, whilst in venous hyperæmia the cortical substance is chiefly affected. Renal hyperæmia occurs in almost all diseases which produce obstruction to the circulation, such as heart and lung diseases, hoopingcough, the acute exanthemata, cholera, tetanus, convulsions, \&c.

Anæmia of the kidneys is either the expression of general anæmia from obstruction to the circulation, or the result of some parenchymatous disease of the organs. 


\section{Hamorrhage from the Kidneys and Hamaturia}

Hæmorrhage from the kidneys, which is not unfrequent in children, appears almost always under the form of ecchymosis, and not as apoplexy of the kidneys, the parenchyma being at the same time healthy.

Hæmorrhage from the kidney and hæmaturia are most marked in purpura and variola hæmorrhagica, scarlatina, croup, pneumonia, scrofulosis, tuberculosis, chronic exanthemata, and such diseases as eczema and pemphigus. A sudden increase in the pressure of the blood caused by forced and fatiguing exercise may also produce them.

\section{Inflammation of the Parenchyma of the Kidneys}

Nephritis albuminosa, s. diffusa, Bright's disease

Bright's disease is of more frequent occurrence in children than is generally supposed, is oftener acute than chronic (acute, more frequent in girls; chronic, in boys), but in by far the greater number of cases it is a secondary, very exceptionally a primary disease (in 324 cases only six times), and it differs in intensity and extent according to its etiology.

\section{Anatomy of Bright's disease (in its more restricted sense)}

(a) Stage of hyperamia.-The kidneys are congested and swollen; the firmness of the parenchyma is diminished; the epithelium of the straight and convoluted tubes is still healthy, or at most slightly clouded, and the Malpighian corpuscles are somewhat enlarged and much congested.

(b) Stage of exudation.-The kidneys are considerably enlarged, their surface is pale and yellowish-grey; the capsule can be easily removed; the cortical substance is strikingly in. creased in extent; and the pyramids are darker in colour, or gradually pass into the yellow of the cortical substance. In the epithelium of the dilated tubuli and of the Malpighian corpuscles an exudation has taken place, together with a deposit 
of a granular cloudy mass and an increase of cellular tissue around the anæmic glomeruli.

(c) Stage of fatty degeneration and atrophy.-The size of the kidneys has diminished; the surface is uneven and nodulated; the capsule separates only with difficulty; here and there yellow spots are to be seen on the grey surface; the contents of the epithelium, and later also the cellular membrane, change into fat-granules and degenerate into an amorphous pultaceous mass.

Other pathological changes, caused either directly or indirectly by Bright's disease, are catarrh of the calyces; inflammation of serous membranes, as pleuritis, pericarditis, peritonitis, pneumonia, bronchitis, amyloid degeneration of the spleen and liver; fatty degeneration and hypertrophy of the heart; more or less extensive dropsy; and once or twice I have found extravasations of blood in tolerably large quantities on the mucous membrane of the stomach and intestines.

Symptoms and course.-Acute Bright's disease, which is, as we have already said, very rarely idiopathic, but, on the contrary, generally dependent on some acute or chronic disease, begins in most cases with disorder of the general health.

A feeling of chilliness, amounting in older children to a rigor, striking pallor of the face, nausea, frequent vomiting, loss of appetite, disturbed 'sleep, lassitude, slight symptoms of fever (pulse 112-120), bleeding at the nose, pain in the region of the kidneys, nearly always occur as initial symptoms of the disease ; whilst those which follow include an altered condition of the urine, frequent desire to pass water, the quantity passed being small, and either of a cloudy red or brown, sometimes even dark like coffee-grounds, and when heated it deposits varying quantities of albumen, and under the microscope presents blood, hyaline and epithelial casts, and thickened epithelial cells. The specific gravity may be either normal or remarkably high, and the reaction is generally acid.

The number and intensity of these changes correspond to the degree of lesion of one or both kidneys.' Sooner or later dropsy, the second characteristic symptom of the disease, appears, varying from slight œdema of the evelids and ankles up to general anasarca. 
In fifty cases of dropsy I found anasarca forty times, ascites twenty times, hydrothorax twenty times, œdema of the brain and meninges fifteen times; œedema of the lungs ten times, dropsy of the pericardium eight times, and œdema of the glottis twice. Dropsy is, however, sometimes absent, and the disease may pursue its course without it, accompanied by the most serious symptoms, either to recovery or death.

The appearance of the dropsy may be quite sudden and accompanied by the most violent nervous symptoms, or it may develop itself slowly and stealthily. The third group of symptoms which generally accompanies Bright's disease includes those of uræmia, which are caused by the retention of the constituent parts of the urine in the blood. These symptoms are those of irritation of and pressure on the brain; headache, which increases at night; vomiting, lassitude, and exhaustion; partial and, still oftener, general convulsions, lasting for hours or even days, and frequently returning after having ceased for some time; grinding of the teeth; delirium, sopor, and coma; disturbances of vision, sometimes blindness, or a feeling as if a mist or veil were before the eyes, sometimes hemeralopia. Death often supervenes, sometimes very suddenly, in the midst of these symptoms; in other cases the uræmic symptoms and the dropsy disappear, the quantity of urine increases, and perspiration and tranquil sleep set in.

It is but seldom that in this stage the disease passes into the chronic form; in such cases the fever disappears, the urine is certainly voided in larger quantities, but still contains albumen; examination with the microscope detects granular, fatty degenerated epithelium, and this may vary in degree.

Bright's disease may, however, sometimes appear from the commencement in the chronic form, in which case it may easily escape notice. In these cases the quantity of urine is never diminished so considerably as in acute cases, and it is of a paler yellow, of lower specific gravity, and contains a great amount of albumen and of epithelium and casts scattered with granules of fatty degeneration.

The symptoms of chronic Bright's disease are excessive anæmia, dropsy, disturbances of digestion, such as want of appetite, dislike of meat, vomiting, cardialgia, and obstinate diarrhœa (uræmic dysentery, according to Treitz); lahoured 
respiration, retarded pulse, headache, disorders of vision, and palpitation of the heart caused by hypertrophy. Sometimes we see these symptoms developed without apparent cause, in other instances they are evidently due to some deleterious influences; and in the course of some chronic cases we meet with exacerbations with all the symptoms and danger of acute parenchymatous nephritis. The duration of the disease varies from a few days to three weeks in acute, several weeks, months or even years, in chronic Bright's disease.

Causes.-Parenchymatous. nephritis occurs in children from the age of eight weeks to fourteen years; I have seen it most frequently between the second and tenth year. Both sexes seem equally liable to it.

It occurs occasionally idiopathically as the result of a blow, wound, fall or exposure to cold; but, as a rule, it is a complication of, or sequela to, some other acute or chronic disease. It most frequently accompanies the acute exanthemata, especially scarlet fever, less often smallpox and measles, scrofulosis, tuberculosis, cases of prolonged suppuration, chronic skin diseases, and diarrhœa, ague, rickets, syphilis, typhoid, \&c.

The diagnosis must be based chiefly on the alterations in the urine, in the examination of which, however, it must not be forgotten that the presence of hyaline casts must be proved ere the diagnosis can be made with certainty, as the epithelial casts are also seen in simple catarrh of the urinary tubuli. The importance of daily and methodical examination of the urine of those patients in whom the development of Bright's disease is to be feared, cannot be too strongly insisted on.

The quantity of the urine depends very much upon the dynamic condition of the blood column, the quantity of fluid consumed, the loss of fluid by other channels, and slight variations in the chemical constitution of the blood, especially in the proportions of the salts and the watery constituents. In general it may be said to be somewhat more abundantly secreted in children than in adults; for, according to Scherer, whilst the adult passes on an average 29.5 grammes in twenty-four hours for every kilogramme of his weight, the child passes $47 \cdot 4$ grammes in the same proportion. According to Pollock, the quantity of urine of the new-born child varies from 250 to 410 cubic centimètres, and its specific. gravity is from 1005 to 1007 . 
In children under six years of age suffering from any serious disease the difficulty of collecting the urine is very great, and an accurate estimation of its quantity can rarely be made, enough being got only to enable us to judge of its chemical and microscopic characters.

The presence of albumen in the urine must not always be regarded as an indication of scrious disease of the kidneys, for it is very usual to find it in any condition which alters the blood-pressure, and in any feverish disease where the temperature is high a transitory albuminuria may be found.

The readiest test for albumen consists in boiling it after the addition of acid, and in small quantity it may be mistaken for phosphates if the acid be not added. To estimate the amount of albumen, Haebler's'method will be found the readiest. 'The specific gravity of the urine is first ascertained, then the albumen is precipitated by boiling and the addition of a few àrops of acetic acid, the urine is filtered, and the specific gravity again ascertained. 'The difference between the two observations multiplied by twenty-one gives the quantity of albumen in a hundred cubic centimètres of the urine.

The usual colour of the urine is clear yellow, though its tint may vary very much. When it is dark brown or coffee-like, its discoloration is generally due to the presence of blood, which may be determined by microscopic examination, as may also other abnormal constituents, as hyaline tube casts, \&c.

Prognosis.-This is always doubtful, is somewhat more favorable in acute than in chronic Bright's disease, although in the latter we notice that the disease may be apparently arrested for months and even years, during which pauses the patient may be in a relatively good state of health. 'The presumed extension, the complications, and the etiology of the disease, must always be considered in the prognosis. Former diseases of the respiratory or circulatory apparatus (disease of the heart, old pleuritic exudations, with adhesion) render the prognosis more unfavorable.

Treatment.-In the acute stage of Bright's disease diuretics are to be avoided, and only gentle antiphlogistic remedies are to be employed, as sinapisms over the kidneys, cold packing over the abdomen, and the internal administration of acids, as phosphoric acid and lemonade; but if there be hæmorrhage 
from the kidney, digitalis, acetate of potash, or juniper may be given, and the alkaline carbonated waters are to be recommended as drinks. The anasarca should be treated by diaphoretics and hydragogue cathartics, or by diuretics if the renal function become re-established. Warm and vapour baths are of much assistance in this direction. When uræmic symptoms occur quinine in two or three grain-doses, with cold packing of the whole body, have been recommended. Frerichs advises flowers of benzoin, and Osborn large doses of calomel. Vomiting may be checked by the use of ice. In the chronic stage a combination of tannin and powdered digitalis leaves will be found useful by internal administration. Warm baths should also be given, and great care taken in preventing any exposure to cold, and in regulating the diet so that it shall be of easy digestion and very nourishing.

\section{Nephritis simplex et Metastatica}

Both kidneys are generally affected in the nephritis simplex, its seat being almost always the cortical substance, the inflammation appearing as circumscribed spots of suppuration, varying from pin-point to the size of a bean or even larger.

Nephritis metastatica may occur in one or both kidneys, and presents sharply defined, wedge-shaped spots, hard and of a yellow colour, softened in the centre; and it may result, as in nephritis simplex, in the formation of pus.

Both of these kidney diseases may occur at all periods of childhood, though I have met with them most frequently in the second year. They are usually due to some urinary lesion, as stone in the bladder, malformation of the penis, as hypospadias, or to some disease of a septic character.

The diagnosis of either of them during life is only proble. matical.

\section{Tubercle of the Kidney}

This is seen in cases of general tuberculosis as small yellowish or grey nodules, seated in the cortical substance and pyramids. Occasionally it is seen as a primary disease in cases where the tubercle is confined to the genital apparatus, and only one 
kidney, as a rule, is affected, that being markedly increased in size, the pelvis dilated, the parenchyma altered to a cheesy yellow mass, softened, and scattered with yellowish-grey nodules.

Secondary miliary tubercle of the kidney produces no perceptible clinical symptoms, and the primary tubercle can be diagnosed only in well-marked cases where there is pain and irritation corresponding to the affected kidney, and when tubercular masses are passed in the urine and recognised by the microscope. In two cases of primary tubercle of the kidney which have come under my notice there existed scrofulous affections in the extremities which had cicatrized.

The course of this disease is protracted, the prognosis unfavorable, and the treatment symptomatic.

\section{Cancer of the Kidney}

This is a rare disease in children as in adults; in the children's hospital at Prague in 100,000 cases it was only seen four times. It is very rare for both kidneys to be attacked, and the left one is more frequently so; in the cases I have seen both kidneys were affected once, the right kidney once, the left twice. Medullary cancer is the ordinary form seen in the kidney, the fungus hæmatodes being very rare.

In the greater number of cases the disease is primary, no cause being discernible; in others it is secondary from extension of the disease from the super-renal capsules, from the neighbouring lymphatic glands or after operation on cancer of other organs.

In two of my four cases the cancer was primary, and secondary in the others; in one from extension of the disease from the mesenteric glands, and in the other it resulted from general carcinosis after extirpation of the eye for cancer.

With reference to the age at which it occurs, Bednar has seen it in an infant of four months, Wöhl in one of nine, and in my four cases the ages of the children varied from three to five years.

Symptoms.-The beginning of the disease betrays itself sometimes, but not always, by pain in the region of the kidney, and 
such disturbances of the urinary organs as frequent desire to pass water, dysuria with the presence of blood and albumen in the urine.' 'These symptoms may, however, be absent especially in those cases where the disease is in an advanced stage, and the parenchyma of the kidney more or less atrophied and supplanted by the growth. 'The most important symptom is a generally uneven, nodulated, immovable tumour, sometimes as large as a child's head, reaching from the false ribs to the crest of the ileum, and inwards to the vertebral column, and occupying a considerable part of the abdominal cavity.

When the tumour develops itself on the right side the liver is pressed upwards, and when on the left side the spleen is displaced in the same direction; the abdomen is generally unsymmetrically distended.

The children become gradually more and more cachectic, with yellowish-white, waxy skin, and finally, after months or even years of suffering, die of exhaustion.

In secondary cancer of the kidney, with general carcinosis, the course of the disease is usually a shorter one.

Diagnosis.-The presence of a hard nodular and immovable tumour in the region of the kidney, with the simultaneous presence of blood and albumen in the urine and a cachectic appearance of the patient, make the diagnosis easy. Mistakes may be made between this disease and the occasional ovarian tumours seen in children and congenital cysts of the peritoneum.

The treatment can only be governed by general directions to treat the symptoms and sustain the strength.

\section{Cystic Kidney; Hydronephrosis}

This may be congenital or acquired, in the former case often presenting considerable obstruction to labour, and in either case it occurs in many various forms. We may find that cavities communicating with one another and with smooth glistening walls have been formed in the tubular or pyramid substance, and that these vary from the size of a linseed or bean up to that of a hen's egg. Distension of the pelvis of the kidney, of the ureters and bladder, also constitute a common variety.

Causes.-Any condition may cause hydronephrosis which 
hinders the exit of the kidney from the calyces or pelvis of the kidney, or any obstruction or an obliteration of the urethra. In a boy three and a half years old I found the cause to be a thickening of the orifice of the left ureter, the canal of the tube above being dilated to the extent of the small intestine. Hillier showed me a very uncommon case in the London Hospital for Children, in which repeated tapping ultimately cured a hydronephrosis.

Hydronephrosis may result from tubercular infiltration of the walls of the bladder, or from their extreme hypertrophy in cases of stone, and from occlusion of the ureters by concretions, or by their compression by external agency, such as enlarged glands. It has been met with in newly born children from congenital stenosis of the orifice of the ureters.

Diagnosis.-The presence of a large, painless, and immovable tumour in the region of the kidney, in which fluctuation is very distinct, may lead to the suspicion of hydronephrosis, but no very certain diagnosis can be made. In renal abscess the symptoms of fever and the presence of pus in the urine from pyelitis, will assist in obviating a mistake between these diseases. Puncture of the tumour has been recommended by various authors, though not a very hopeful proceeding.

\section{Concretions in the Kidney}

'These are met with in two forms which are not separable ætiologically, the infarctus of new-born children, and larger concretions varying from the size of a pin-liead to that of a pea or cherry-pip.

The uric acid infarctus is found in the bodies of children who die within the first three weeks of life, as a regular and welldefined streaking of the pyramids, of an orange or golden-yellow colour. On microscopic examination the reddish-yellow crystals of uric acid can be recognised between the epithelial layers of the straight tubules. The infaretus originates, according to Virchow and others, in a more or less prolonged insufficiency of the respiratory process after birth. When the respiration becomes completely established and the quantity of water passed by the kidneys becomes increased, the infarctus is 
loosened and pushed outwards, and it is found on the clothing as red granules; or some of it may be retained in situ and lead to the formation of the larger concretions.

In more advanced childhood renal concretions are sometimes found under certain hereditary or endemic influences, or, in the course of some diseases by which the secretion of the kidneys is diminished in quantity, as, for instance, in chronic intestinal catarrh, or when alkaline decomposition of the urine occurs within the kidney.

During life these concretions, especially those of the infarctus, produce no very distinct symptom; but in some cases, especially in children over three years of age, the condition may be suspected from the presence of grit in the urine accompanied by straining and pain on passing water, or even reflex spasm of the urethra. If the concretions are retained in the bladder they increase in size there, and may remain until removed, or they may be impacted in the urethra and may require to be assisted in their passage outwards.

Suppurative pyelitis, with ulceration of the pelvis of the kidney, is sometimes produced by the retention of the calculi there.

The best treatment consists in the administration of abundance of fluid, especially of alkaline waters, as soda and seltzerwater, an increase in the vegetable constituents of the diet and warm baths to assuage pain.

\section{Floating Kidney}

This is a very rare abnormality, and one I have seen only three times; once in a boy ninc years old, and in two girls respectively six and ten years of age. In all three it was the right kidney which was found to move low down in the abdomen and across the median line to the left side, where it could be felt as a hard, smooth, bean-shaped tumour, free from pain, and easily moved. These children suffered from vomiting, giddiness and headache, loss of appetite, colicky pains, and in two cases there seemed to be attacks of partial peritonitis lasting about fourteen days.

Supporting the abdomen by a binder may give relief to any sense of discomfort, and all violent exercise should be avoided. 


\section{Congenital malformations of the Bladder}

The most frequent of these is ectopia vesicæ, a deformity which is found as often in boys as in girls. In these cases the umbilicus is at an unusually low level, the genital sinus is not closed, and between the umbilicus and pubis there appears a roundish and flattened, dark red tumour, its surface consisting of mucous membrane, and which may be recognised on examination as the posterior wall of the bladder by the ureters opening upon it. Rudimentary development of the sexual organs is a usual accompaniment of this arrest of development.

In the cases which have come under my care the children have usually been weakly and have died early, and in only one case has the child grown up.

The exposed mucous membrane becomes dry and skin-like, especially at the upper part, whilst at the lower surface both skin and mucous membrane suffer from excoriation and ulceration from the constant dribbling of the urine.

A radical cure of this deformity is hardly possible, and the patient's condition can only be remedied by a careful attention to cleanliness and keeping the parts smeared with glycerine and oxide of zinc, or, as the child grows, by some apparatus of silver or vulcanite applied so as to catch the urine and protect the parts. ${ }^{1}$

\section{Catarrh of the Bladder; Cystitis}

In children inflammation of the bladder is frequently met with in the simple catarrhal form, and occasionally as a croupodiphtheritic disease. It may be the result of blows, or stone, or some foreign body in the bladder; of the administration of some irritating substance, as cantharides or some terebinthinate; from the extension of the inflammatory process from some other organ, or of exposure to cold, or it may occur in the course of some zymotic disease.

In simple acute cystitis the vesical mucous surface becomes markedly injected, swollen, and the secretion is increased in quantity; whilst in the chronic form the membrane becomes of

1 The successful operations by Prof. John Wood show that ectopia vesica is not quite so incurable as Dr Steiner believes.-(L. T.) 
a slaty-grey colour, is covered with mucus and pus, and scattered with spots of ulceration. Perforation of the bladder is of the rarcst occurrence.

In the croupo-diphtheritic cystitis the fibro-cellular exudation appears in a membranous form in isolated spots or streaks.

Symptoms.-The severity of the symptoms is in proportion to the acuteness of the course of the disease. There is usually some degree of fever, pain over the symphysis and in the region of the perinæum; a continuous straining to pass water, which comes away in small quantities, followed by a few drops coloured with blood. In chronic cystitis the urine is always muddy, and either of a neutral reaction or faintly alkaline, and it contains a large quantity of mucus and pus-corpuscles, which fall with other sediment to the bottom as the urine cools and settles.

In croupo-diphtheritic cystitis the pain in the bladder and the ischuria are very severe, the fever is very marked, and vomiting is usually troublesome, and shreds of the false membrane are often passed in the urine. This latter sign I saw very well marked in a boy six years old, in whom this form of cystitis supervened in the third week of an attack of typhoid.

The course of cystitis is varied according to the exciting cause, being sometimes rapid and favorable in its ending, sometimes chronic, obstinate, and full of anxiety, the latter being especially the case when it is due to the presence of a calculus.

Treatment.-The most important indication is for the removal of the cause, especially in the case of stone. For the local pain warm baths and poultices over the bladder are very useful; and opiates, either alone or in combination with calomel, are beneficial. In membranous cystitis and in the chronic form lukewarm injections, containing in the former case chlorate of potash and in the latter tannin or some other astringent, will be found very serviceable. In the case of retention the bladder must be periodically emptied by the catheter.

If the catarrh be the result of exposure to cold, vapour baths, repeated at intervals, will be found very useful, and when there is much straining warm hip-baths will afford great relief; when the pain is very severe morphia must be given either by suppository or subcutaneous injection. In croupous cystitis solutions of chlorate of potash should be injected into the 
bladder, and tannin may be given internally with decoction of uva ursi. When there is retention of urine the catheter must be used methodically. In every kind of vesical inflammation absolute rest is imperative, the diet must consist of mild nutritious food, and the drink of fresh cold water, administered often in very small quantities.

\section{Tubercle of the Bladder}

Tubercle of the mucous membrane of the bladder is usually associated with tubercle of some other part of the urinary or sexual organs, and is most frequent amongst males. 'The pathological appearances include small, grey, or yellowish-grey nodules perceptible to the eye, scattered over the mucous membrane; small lenticular ulcers, or some of larger size with mucous margins, and evident loss of substance; small yellow or yellowish-brown, cheesy masses of tubercle; and hypertrophy and dilatation of the bladder.

The symptom-complex is that of chronic cystitis, and in one case which I saw there had been incontinence of urine for several weeks before the other symptoms appeared.

Diagnosis.-This must be based on the local symptoms and any indications that may he obtained from tubercular deposits elsewhere. The prognosis is always unfavorable, and the treatment can be only symptomatic and palliative. Opiates by the mouth and rectum or by subcutaneous injection, strong and easily digested nutriment and demulcent drinks constitute the limit of our efforts.

\section{Stone in the Bladder}

This disease is very frequent in children, the great majority of the children being boys; and it may be said, generally, that the chemical and physical characters of the calculi, their pathogenesis and symptomatology, are very much the same as in adults.

The following facts concerning stone in the bladder have been drawn from the observation of Bokai and Neupauer ('Jahrbuch. der Kinderheilkunde,' 18r2). 
The disease has been found in children three or four months old, but the greatest number of cases occurred between the ages of two and seven. The size of the stones varies considerably, as did their weight, the latter seeming to have no proportion to the former, but to the composition of the calculus. The largest weighed $5 \% 0$ grains and the smallest, passed from the urethra, was one fourth of a grain. The heaviest stones are those of uric acid or oxalate of lime. In about half of the cases the stones had a smooth surface, whilst the others were more or less rough, and their shapes were very various.

The internal structure and lamination of calculi vary very much, some having a cut surface like an agate, others having layers of hard alternating with layers of soft porous material or with dendritic structure; whilst others display no lamination whatever, their fracture resembling that of a peach kernel. In most calculi will be found a nucleus the structure of which is homogeneous and laminated, being non-laminated in very exceptional cases, and sometimes more than one nucleus may be found in the same calculus.

Heschel has stated that the most numerous and the largest stones are those composed of uric acid and urates of ammonia, soda, magnesia, and lime. They are usually brown, rarely white, frangible, and usually have a smooth surface, though sometimes it is tuberculated. Phosphatic calculi are composed chiefly of the phosphates of lime, magnesia, and ammonia; they are white, hard, and brittle, and have usually a smooth surface. Some stones consist of alternate layers of uric and phosphatic deposit. The oxalate calculi are very hard, dark brown in colour, and are tuberculated on the surface or even acuminated.

Vesical calculi usually have their origin in renal concre. tions, from the infarctus and hereditary tendency is often well marked, especially in delicate and insufficiently nourished children. Bokai considers that the frequency of stone in children is due to the difficulty they have in passing the solid uric acid.

Usually there is only one stone, though there may be two or more. The more common varieties are three in number; the uric-acid stones, which are the largest, are of a brownish colour, brittle and smooth on the surface; the phosphatic, which 
are white, and may be either firm in consistence or crumbly; and the oxalate calculi, which are of a dark brown colour, nodular on the surface, and often spiked.

Causes.-The origin of vesical calculus in renal concretions and infarctus has already been mentioned, and there can be no doubt that there is an hereditary tendency to them, and they are very common in weakly and badly reared children.

Symptoms.-These include irritation and more or less pain in the region of the bladder, increased on movement or shaking of the body, and ceasing when the patient is quiet in the recumbent posture; sudden interruption of the stream in micturition and painful straining when the bladder is emptied, erections, dragging at the foreskin with the fingers, retention and incontinence of urine, and the passage of blood in it, together with the symptoms of vesical catarrh already detailed. The absolute sign is the detection of the stone by the sound.

There are often some marked general symptoms, such as continued fever, sleeplessness, and, if there are any indications of inflammatory action in the neighbourhood of the bladder and kidneys, the prognosis is rendered somewhat unfavorable.

The course of the disease is essentially chronic. Sometimes stones of a considerable size are passed by the urethra, and they have also been found to escape into the rectum and vagina by ulceration of the walls of the bladder.

Treatment. - When the presence of a stone has been definitely ascertained, and that cannot always be done at the first examination, the only remedy is the operation of lithotomy. In children the results of this proceeding are much better than in adults, and it is much to be preferred in the former to lithotrity.

Out of 100 cases of lithotomy Guersant has lost fourteen; eight from the immediate effects of the operation and six from intercurrent diseases; out of forty cases of lithotrity he lost seven. When there is a tendency to the formation of gravel and deposition from the urine, as $I$ have seen in several children from ten to twelve years of age, the mineral waters of Karlsbad and Vichy will be found to be useful. 


\section{Enuresis, Incontinence of Urine, Bed-wetting}

This may be only a symptom, or it may constitute an independent disease. In the former case it is seen in children who are idiotic or of deficient intellect, in the course of diseases of the brain and bladder, and also in other serious affections; if the latter, the incontinence occurs only during the night, usually the bladder being emptied only once, and that during the first three hours of sleep.

The cause would seem to exist in deficient sensibility of the nerves of the bladder, which is such that the irritation of the distension of the bladder is sufficient to excite the motor apparatus, but not to produce consciousness, and this occurs the more if the sleep be very deep. This anæsthesia is sometimes the expression of general debility, so that very generally though not invariably the enuresis is found in scrofulous and rickety children ; or it may be simply local, and the indication of some weakness of the bladder or its sphincter, unless we can ascertain some more tangible cause for it.

I have some reason to believe that this affection is hereditary, and I have several times seen the majority or all the children in a family affected. In some cases it arises merely from laziness and bad habit, and these can easily be relieved by insisting on a better behaviour.

Prognosis.-Thongh this affection involves no risk to life, it is excessively troublesome both to the patient and the friends. It generally gets well as the child grows, either at the period of the second dentition or at puberty, though in some cases it continues after the latter period. During attacks of such diseases as the acute exanthemata the incontinence generally ceases, to recur again after recovery.

Treatment.-If it arises merely from laziness and a bad habit the child must be judiciously punished ; but if it be the expression of some morbid condition, that must be investigated and receive its appropriate treatment. In scrofulous and rickety children cod-liver oil, iron, and iodine, must be given; and in cases of anæmia, iron and quinine. For local treatment I have found cold and aromatic hip-baths, and the retention of a catheter in the urethra for some time, very useful. The extracts 
of belladonina (in doses of $\frac{1}{10}$ to $\frac{1}{2}$ a grain) twice daily and nux vomica $\left(\frac{1}{30}, \frac{1}{20}\right.$, to $\frac{1}{8}$ of a grain) have been recommended, but I have not seen any definite results from their use. It is advisable to have the child roused during the early hours of its sleep that it may empty its bladder, but this must be regularly continued for some time with care and patience; the child also should have only dry food for supper and very little liquid.

No mechanical contrivance, however ingeniously and carefully contrived, should ever be used for compressing the penis or neck of the bladder, for such can never prove much more than an instrument of torture.

\section{Hypospadias and Epispaclias}

Hypospadias includes all malformations of the urethra by which it is made to open at the under surface and not at the end of the penis, in the more common and slighter cases the opening being found at the base of the glans, and in the more severe either at the root of the penis or even behind the scrotum. In some cases the scrotum and perinæum are completely cleft, and the bladder opens directly, without urethra, into the aperture.

Epispadias is characterised by the opening of the urethra on the dorsal aspect of the penis, the cleft extending over the glans above or along the whole of the penis, ending, in some cases, in an extroverted bladder. Epispadias is much less common than hypospadias.

Many operations have been contrived for these deformities, especially by Gerdy, Nélaton, Bichard, and Guersant; but the results have not been good.

\section{Cellular Adhesion of the Prepuce to the Foreskin}

This condition is more frequently of physiological than of pathological import, and has been recently investigated and described by Bokai. In boys not suffering from phimosis, or even in whom the prepuce is not wide enough to coyer the entire glans, the foreskin may occasionally be found incapable of being retracted behind the corona, even if considerable 
exertion be made, and in these cases union will be found to have taken place between the glans and the mucous surface of the foreskin.

Bokai has described three varieties of this adhesion, in the first of which it extends from the corona backwards, as seen in cases of hypospadias and in circumcised Hebrew children; in the second the adhesion extends backwards from the anterior half of the glans, the prepuce being normal in width and length ; whilst in the third the union extends from the apex of the glans to the retro-coronal sulcus, the foreskin is very meagre, and in new-born children suffering in this way the meatus urinarius may even be found occluded.

In most cases the deformity may be quite relieved by forcibly retracting the foreskin and breaking down the union by a blunt probe.

\section{Congenital Phimosis}

An abnormally narrow orifice of the prepuce, with either excess or deficiency in the quantity of its skin, constitutes the most common malformation of the penis. In the majority of cases the deformity remedies itself as the child grows, but the foreskin may be preternaturally long and the orifice narrow, so that micturition is painful and impeded, and the retained secretion of the glans gives rise to balanitis, ulceration and even adhesion of the two opposing mucous surfaces.

If the constriction is allowed to continue, the development of the penis seems to be arrested to some extent, especially of the glans, compressed, as it is, by the constricting prepuce ; and the collection of smegma, liable to putrefaction, often gives rise to inflammation and excoriation of the mucous surfaces, followed sometimes by adhesions. When the degree of phimosis is extreme it may constitute an obstruction to the passage of urine, causing considerable discomfort and suffering to the children, and exciting sometimes the behaviour seen in cases of stone in the bladder, when the penis and foreskin are dragged and pinched to obtain relief. Very often, though not always, this narroiving of the foreskin disappears entirely as the child grows. 
Treatment.-Simple incision at the orifice or extending upwards, or even excision of a part of the foreskin. Distension of the constricting part by sponge tents may be tried, but it is not easily done in children, and circumcision may always be recommended as the best remedy. This operation is not, however, altogether without risk, for I have known the ritual operation followed by troublesome hæmorrhage, and even by fatal erysipelas.

\section{Inflammation of the Foreskin; Balanoposthitis}

This gives rise to itching, burning, and stinging pain increased on passing water. The foreskin and anterior part of the penis is swollen and red, and between the glans and the foreskin a layer of smegma or of thin, greenish-yellow, purulent discharge collects. The œdematous swelling produces phymosis and excoriation of the mucous surfaces, followed sometimes by their cohesion. Abscess and gangrene of the prepuce may be met with in some cases.

The causes of this affection may be found to be accumulation of smegma, from inattention to cleanliness, masturbation, injuries, lodgement of intestinal parasites, eczema of the genitals, \&c.

A little attention to cleanliness and a lotion of acetate of lead will always get these cases rapidly well. The prepuce must be retracted by an operation if necessary, and the smegma periodically removed. When excoriations have occurred some oily application must be made, to prevent union of the surfaces.

\section{Paraphimosis}

When a narrow prepuce is retracted over the glans, either by design or accident, and retained there, paraphimosis results; and the strangulation of the glans which ensues is often induced also by the application of pieces of thread, or wire, or rings. The swollen and odematous prepuce and the bluish-red distorted glans produce an appearance of the organ which is alarming. Micturition becomes painful, and at the point of strangulation there is some purulent discharge, especially if the strangulation be the result of some artificial constriction, and 
deep ulceration, or even necrosis and gangrene, are not unfrequent results.

The treatment of recent paraphimosis is very simple, consisting only of reposition, which is easily accomplished by compressing the glans between the fingers, and exerting traction on the prepuce, first placing a little oil round the corcna. If the displacement be of some standing, some cold or astringent applications must be applied for some little time before reduction be attempted; and if after this the attempt do not succeed, the constricting ring of the prepuce must be notched.

If the constriction be due to some foreign substance, that muist, of course, be cut and removed.

\section{Masturbation}

Boys are apt to excite precocious erections by various manipulations of the penis, accompanied by orgasm and the ejection of a slimy fluid. Girls, who are much less given to these practices, excite themselves by rubbing the clitoris with the finger or some instrument, such as a pencil or a candle. This precocious sexual excitement very frequently, though by no means invariably, induces a diminution of both bodily and mental vigour, the peculiar freshness of childhood disappears, the skin becomes blanched and wrinkled, the child becomes listless and easily tired; the gait becomes uncertain, the facial expression becomes old, sexual, and apathetic; the eyes dull, watery, evasive, and surrounded with a dark ring, especially on the lower lid; the nostrils dilating; and the penis will be found to be disproportionately developed, and its prepuce very moveable. In girls who have practised masturbation the vulva will be found gaping, the clitoris swollen and reddened, the nymphr hypertrophied, and the hymen very often ruptured.

I have often had occasion to observe that these practices begin very early in life, as I have seen them in children one and two years of age, especially in those who, after weaning, take to suck their fingers and the corners of their linen and sheets. In these it is not uncommon to find erections, most frequently during sleep, or shortly before the child awakens. Marjolin has stated that such conditions are to be met with 
even in children at the breast, but I am not in a position to confirm his assertion.

The most frequent cause of this vice is mixing with children already addicted to it, especially in boarding schools and similar institutions, it being much more uncommon in children who are brought up at home. Nurses of loose morals are also to blame for a good deal of it, whilst among the intrinsic causes may be mentioned collections of smegma behind the prepuce, and displacement there of worms wandered from the child's rectum; also chronic skin eruption on the genitals, especially chronic eczema. There are many predisposing causes which ought to be avoided, such as the use of feather beds, too strong diet, especially meat suppers, and the perusal of immoral and exciting books and pictures.

Treatment.-This is a very difficult matter, requiring much caution and patience, and must consist chiefly in carefully watching the child. Moral lectures and vivid representations of the ill results likely to follow from such practices do not seem to have any good results, the best plan, according to my own experience, being to make the practice of onanism almost impossible by carefully watching the child night and day, or by making it painful by some slight operation. The bed which the child occupies should be a hard mattress, the bedclothes light and of wool. During sleep the child's hands should not be allowed to remain under the bedclothes, and if he has to go to the closet care must be taken that he is not allowed to remain there long. Children who are known to practise masturbation should not be allowed to remain in an institution where they may corrupt others. Masturbation may be made painful by some such process as circumcision in boys, and the occasional cauterization of the nympha with nitrate of silver in girls, or, according to Gross, by making small incisions into the clitoris.

The use of sewing machines, which has become of late so prevalent, seems to me to have an evil influence in this direction, especially for young girls, and I have heard several times from women expressions of opinion in this direction. 


\section{Cryptorchia}

Though as a general rule the testicles are found in the scrotum at birth, yet occasionally cases are met with where one or both have not descended, and the retained testicle may remain in the abdominal cavity till puberty, or it may remain there permanently, becoming atrophied.

Care must be taken not to confound an undescended testicle with an enlarged gland or a hernia, should any treatment be thought necessary for one or other of these conditions.

The testicle has also been found descended into the perinæum and on the inner side of the thigh, but such displacements do not require treatment.

I have met with a very unusual case, where there were three testicles in a boy five years old, one being enclosed in the right half of the scrotum and two in the left, the third being on a higher level than the other two, and somewhat smaller.

\section{Hydrocele}

This consists of an abnormal collection of serum in the tunica vaginalis, and is a somewhat common affection in childhood, for in the Prague Hospital 230 cases have come under observation in ten years.

It is rarely acute, most generally chronic in its course, may be either congenital or acquired, and it may affect either the tunica vaginalis or the spermatic cord.

\section{(a) Hydrocele of the Tunica Vaginalis}

The effusion occurs more frequently on the left side than on the right, but sometimes on both; and produces an oval peashaped tumour, varying from the size of a pigeon's to that of a hen's egg, with a distinct limitation at the inguinal opening, translucent and fluctuating on touch, and giving a dull note on percussion. The testicle is most frequently found on the posterior wall, rarely at the lower part or in the middle of the tumour. The contents of the sac consist of a thin, clear yellow serum, containing albuminates and fatty cells, and sometimes 
blood and cholestearin. If the hydrocele be congenital, the canal passing between the tunica vaginalis and the general peritoneal cavity may be more or less patent, and the contents of the sac may then be pressed back into the abdomen. This condition is often associated with hernia.

Diagnosis.-From the above-mentioned characters the diagnosis of hydrocele of the tunica vaginalis is easily established, the chief difficulty being to distinguish it from inguinal hernia, more especially if the serous canal is patent and there is hernia coincident with hydrocele. In such cases the manner in which the tumour recedes into the abdomen, whether slowly or suddenly, whether accompanied or not by gurgling, the character of the percussion note, the translucency or opacity of the tumour, and the presence or absence of the testicle will make the nature of the swelling clear.

Treatment.-In new-born children and during the first years of life hydrocele usually disappears spontaneously, and it is seldom that anything is required to be done beyond the application of some resorbent, such as an ointment of digitalis and iodide of potash.

If the parents are impatient the sac may be punctured, and if the effusion returns, the radical cure may be effected by injection with tincture of iodine. In puncturing the tumour care must be taken to avoid the testicle and any large vein which may be visible. When the hydrocele is congenital, that is, when the serous canal is patent, the radical cure is contraindicated, as the resulting inflammatory action may spread over the peritoneum, and tapping is the most which should be attempted.

\section{(b) Hydrocele of the Cord}

If the serous canal of the cord be not obliterated, but closed at each end, and the resulting cavity be distended with serum, the condition is known as hydrocele of the cord. The tumour thus formed is generally somewhat spindle-shaped, situated in the plane of the spermatic cord, and extends from the depressed testicle to the inguinal opening, is about the size of a pigeon's egg and translucent. As a rule the cysts are single, but sometimes they are multiple.

They may easily be cured by tapping, either simple or fol- 
lowed by injection of iodine. Guersant uses setons passed through the cyst.

\section{Cellular Atresia of the Vulva}

Under this name Bokai has described a condition in which the labia minora are agglutinated throughout more or less of their extent, so that when the labia majora are separated the vestibulum vaginæ is not visible. Of thirty-nine cases observed by Bokai twenty-four had the atresia complete, and in the remaining fourteen it was partial. I have seen twelve cases, and in seven it was complete. The union may be very slight between the opposing edges, or it may be more intimate, but in those which have come under my notice it has been undone very easily and without any bleeding. As a rule, it gives rise to no symptom, but in an extreme case it might cause retention of urine.

This curious condition seems to have some analogy with the cellular adhesion between the prepuce and glans already mentioned, and Bokai considers it is due to a want of hardening (Verhornung) of the epithelial surface of the labia, which become adherent, therefore, from their apposition. It is less common than the analogous union between the prepuce and glans in the male, and will become undone spontaneously if left alone, especially if the atresia be not complete. If there be any interference with the passage of the urine surgical relief must be given by rupturing the united edges by a probe, and dressing the parts with some greasy application to prevent reunion.

\section{Vulvo-vaginitis}

This constitutes the most frequent and important of the diseases of the genitals which occur in childhood, and it is met with of the following varieties:- $(a)$ catarrhal or erythematous, $(b)$ phlegmonous, (c) croupo-diphtheritic, $(d)$ gangrenous, $(e)$ syphilitic, and $(f)$ exanthematic. The last two belong exclusively to syphilis, the acute exanthemata and chronic skin diseases, so that now we shall consider only the first four. 
(a) Catarrh of the Genital Mucous Membrane; Vulvo-vaginitis Catarrhalis ; Fluor Albus

The most prominent symptoms are a reddening of and increased secretion from the genital mucous membrane, the latter consisting sometimes of a thick yellow or greenish-yellow fluid, and sometimes it is of a thin muco-purulent character. There is generally pain of an itching and burning character, especially during and after the passage of urine, a peculiar gait, excoriations of the mucous membrane, sometimes slight fever and a sympathetic enlargement of the neighbouring lymphatics.

Causes.-These may be constitutional or local, if the former the most important being the scrofulous or tubercular dyscrasy. Of the local causes the chief are,-want of cleanliness, collection of the decomposed secretion of the sebaceous and mucous glands; mechanical irritation of foreign bodies in the vagina, the presence of ascarides, and the practice of masturbation. It may occur in children of any age.

The course of the disease may be cut short in two or three weeks, but it is very apt to become chronic and last for months or even years, with alternate improvement and remissions, especially when it occurs in scrofulous and anæmic children.

Treatment.-This must be essentially causal, for the removal of the cause alone will often suffice to effect a cure. When the disease is acute, absolute rest, cold hip baths, and lotions of acetate of lead or some other astringent should be advised. Great attention to cleanliness is absolutely necessary, and we must secure the absence of any foreign body. When there is some constitutional influence at work the appropriate treatment must be employed.

\section{(b) Vulvo-vaginitis Phlegmonosa}

This variety is generally found in the acute form, and is ushered in by shivering and subsequent fever, violent local pain, which at first is of a stinging character, redness of the skin in the neighbourhood of the vulva, in which afterwards may be discovered a soft, fluctuating spot, from which pus may be evacuated. In some cases the inflammation stops short of 
suppuration, and slowly resolves itself or gives rise to chronic ulceration. The causes of this variety include blows, exposure to cold, want of cleanliness, and neglected catarrhal vaginitis.

In the treatment absolute rest must be enjoined with antiphlogistic remedies in the first stage, especially cold applications; but when suppuration has become established warm poultices must be applied and the abscess opened. When there is a tendency for the disease to become chronic, constitutional remedies, such as cod-liver oil, iodide of iron, and iodised mineral water must be employed, in conjunction with the local use of iodide of potash ointment or of the tincture of iodine.

\section{(c) Vulvo-vaginitis Diphtheritica}

This generally occurs as a part of some general disease, especially, of course, diphtheria, and also the acute exanthemata, as scarlet fever, measles, smallpox, typhoid, \&c. Less frequently it has only a local significance, occurring primarily or secondarily to some other disease of the genitals. 'The exudation appears on the mucous membrane, and resembles what has been already described as occurring in the throat. As a rule, it softens and disappears, though in severe cases it may pass into gangrene. The neighbouring glands are often swollen and tender, and the ulceration which follows the disappearance of the exudation may extend to the external genitals and perinæum. In cases of recovery, if care be not taken, the opposing surfaces may become united. The general symptoms include loss of appetite, fever, emaciation, and dry skin. It may occur at any period of childhood.

The prognosis is not always favorable, especially if it be an expression of general diphtheria. Under other circumstances the character of the attack, the constitution and circumstances of the child must be taken into account in giving an opinion; and its appearance amongst hospital patients must always be regarded as a serious matter.

Treatment.-According to circumstances this may either be simply local, or it may require to be directed against the general disease. In the first stage the application of a strong solution of chlorate of potash or lime water will be found useful; as the disease advances cauterization with nitrate of silver or muriatic 
acid may be tried; and if gangrene threatens, some disinfectant, such as spirit of camphor or a solution of chloride of lime, must be applied.

The general treatment of diphtheria has already been discussed. If recovery takes place, union of the opposing surfaces must be guarded against by the insertion of pieces of oiled lint.

\section{(d) Vulvo-vaginitis Gangrenosa}

This is generally only the last stage of some previous inflammatory affection of the genitals, occurring almost always in children much exhausted by severe disease, such as typhoid, diphtheria, \&c.

The gangrene appears as dark discoloured spots, which rapidly extend in size and depth, leading often to very extensive destruction of tissue. The febrile symptoms are generally severe, and gangrene of some other part, especially of the face (noma), is not an unfrequent concomitant. Death is the most common result, separation of the gangrenous masses and cicatrisation of the resulting sores being unfortunately rare. I have, however, seen it once, where gangrene occurred in the course of typhoid, and destroyed the whole of the left labium.

This disease affects children most frequently between the second and sixth years of age, though $I$ have seen it in a suckling three months old.

Treatment.-The local treatment must have for its object the cutting short of the gangrene, for which purpose cauterization with the mineral acids or the actual cautery may be made, or some of the many antiseptics may be tried, such as camphor, chloride of lime, permanganate of potash, \&c., and great attention must be paid to keeping the parts clean. The general treatment must be nutrient and stimulant.

\section{Swelling and Inflammation of the Mamma; Mastitis}

Occasionally we see, in either sex, a painful swelling of one or both breasts in young children, not confined to those who are weakly or badly nourished, and accompanied by the exudation of milky fluid from the nipples. This swelling may last for a week or two, and then usually disappears. In one case I 
saw it associated with vaginal hæmorrhage, though there was no distinct evidence of any connection between the two circumstances.

This swelling may be idiopathic, or it may be due to some injury, generally the fault of the nurse, and the inflammation may extend to suppuration and the formation of an abscess, or to a chronic induration of the tissues of the breast. The process of suppuration will give rise to its characteristic symptomspain increased on pressure, fever, restlessness, and even convulsions, and sometimes there may be indications of erysipelas.

As a rule, the course of this disease is free from risk, at the most atrophy of the gland following the formation of an abscess, involving its uselessness for the purposes of nursing in afterlife, in the case of a female child.

Towards puberty growing girls are often troubled by a painfulness of one or both breasts, which lasts only a short time and is significant of the general physiological change.

Treatment.-Simple swelling of the gland requires no interference beyond some greasy and warm application. If suppuration threatens, poultices must be applied; and when the abscess has formed it should be opened early, as destruction of gland-tissue may thereby be averted.

Any resulting chronic induration may be treated by iodine applications.

\section{Appendix}

\section{Diseases of the Umbilicus}

The development of the umbilicus is intimately associated with that of the abdominal walls, intestines and the structures of the cord, and it is apt to participate with them in any arrest of development. After the child is born and separated, if the pulmonary circulation becomes fairly established, the stump of the cord soon dessicates and ulcerates off, the time of its separation depending upon the condition of the cord and of the child, a thin cord falling off more readily than one thick and rich in jelly, and in a well-developed child than in one which is weakly. 'The separation generally takes place between the third and 
tenth days. The stump should be carefully wrapt in soft linen. rag, and the washing and dressing of the child so managed that it should not be dragged off prematurely.

\section{Fissura abdominalis}

When this is complete the cleft extends from the manubrium sterni, or it may vary in extent, being limited to the abdominal walls, or between the umbilicus and sternum. The cleft generally occupies the median line, and it may be complicated with eventeration, ectopia resicæ, formation of cloacæ (cloaken bildung), spina bifida, and other malformations, which are more or less incompatible with life.

\section{Hernia funiculi umbilicalis}

This depends upon a deficiency in the closure of the abdominal wall at the umbilicus, and the sac may be composed only of peritoneum and amnion, the contents, in extreme cases, including sometimes the greater part of the intestinal canal, and even part of the liver, stomach, and spleen. In such cases life is impossible, but when only a small knuckle of intestine is included a cure may be effected. This malformation must not be confounded with the ordinary umbilical hernia, which occurs after the separation of the cord and cicatrisation of the cord. ${ }^{1}$

1 I have seen only one case of this malformation, and I did not succeed in benefiting the patient; but a further consideration has induced me to believe that if 1 had been somewhat more heroic in my treatment the case would have had a more satisfactory ending. I saw the child in consultation with my frierd and colleague Dr Charles Warden, and it presented at the umbilicus a huge mass, very like a large penis affected with a very severe gonorrhœa. It evidently containcd gut, and from several cracks on the covering it was clear that the tissue of the cord, plus peritoneum perhaps, though this is doubtful, constitnted the sac. In our effurts to reduce the hernia we ruptured the sac, and the bowels protruded. We then saw that they were adherent everywhere to the sac, but that the adhesions did not bleed when torn. Neither of us had ever seen such a case, and I could find no advice for treatment under such circumstances anywhere. We, therefore, earefully covered the intestines, and left the case to nature, who ended it in ten days. I now believe that if we had boldly picked off az much of the sac as we could, returned the intestines, and stitelsed up the aperture, we should have done what was right, and might have saved the patient.-L. T. 


\section{Ectopia vesica}

has already been treated of.

\section{Amnion-navel}

This has been met with but seldom, and, according to Widerhofer, consists of an abnormally broad insertion of the sheath of the cord, and a corresponding defect in the skin of the abdomen. The children so affected do not live.

\section{Sarcomphalus, fungus umbilici, raspberry-navel}

This is a broad-based spongy tumour which forms between the fourth and tenth days from the exuberant granulations on the umbilicus after the cord has dropped off, the surrounding skin being either normal in appearance or somewhat reddened. Left to themselves these tumours generally disappear after lasting some time; but I have seen them recur, and in one case this happened, and the tumour existed until the child was four years old.

The treatment may consist in cutting the mass off with scissors, and touching the stump with caustic or perchloride of iron, but in mild cases the cutting may be dispensed with.

\section{Ompthalitis}

The granulating surface of the umbilicus may become the starting point of a very destructive inflammatory process, which may be excited by want of due attention to cleanliness, to some chemical or mechanical irritation, or to pyæmic or other septic infection.

Under some such influence the wound takes on an ulcerative action, suppurates or becomes covered with a croupo-diphtheritic exudation, secreting pus or purulent ichor; the surrounding skin partakes in the action, and becomes erythematous, erysipelatous, or even involved in a phlegmonous inflammation, and suppurative peritonitis, perforation of the abdominal walls, gangrene, hæmorrhage from the umbilicus, or some constitu- 
tional condition, such as pneumonia, pleurisy, jaundice, or thrombosis may occur to complicate the case.

This disease very seldom has a favorable issue, especially if complicated; in such a case the fatal course being usually very rapid, and accompanied by the symptoms proper to the complication.

The wound left by the separation of the cord should be carefully attended to so that it be kept clean, and in the event of any ulcerative action some such lotion as a weak solution of chlorate of potash should be used as a dressing. If any ichorous discharge makes its appearance, some disinfectant application, as a solution of chloride of lime, will be found best; if erysipelas supervenes, olive oil or Goulard's extract will, perhaps, be serviceable ; and in the event of suppuration, poultices should be applied. If the child be not taking the breast, the milk must be administered by means of a spoon; and if the case seems pyæmic, quinine in doses of one sixth to half a grain may be given.

\section{Thrombosis and inflammation of the umbilical vessels}

Thrombosis of the umbilical vessels is a process which may be simply physiological or partly of pathological importance, and in some cases it has an intra-uterine origin, as clots are to be found in the convoluted vessels of the cord, more frequently in the arteries than in the veins, Wrany having found in the investigation of 120 infants, thrombosis twenty-three times in the arteries and three times in the veins. The thrombosis may, however, extend farther than the vessels of the cord, either from the vein into the hepatic radicles of the portal vein or through the ductus venosus into the lower vena cava, or into the arteries as far as the fundus of the bladder, or even into the cavity of the pelvis. The cause of such thrombosis is not yet very clear.

The clots may undergo further change by softening and ichorisation, leading to phlebitis or atcritis, with swelling and infiltration of the surrounding tissue; and, by the scattering and impaction of the softened mass, to the many complications which have already been described as due to such causes, and amongst which may be mentioned general pyæmia. 


\section{Gangrene of the umbilicus}

Any inflammatory condition of the umbilicus, especially omphalitis, may, under unfavorable circumstances, result in gangrene; and this is especially apt to be the case in badly managed and overcrowded lying-in and foundling hospitals, and in weakly children or in those already suffering from some other disease. The tissue of and round the umbilicus becomes discoloured to a varying extent, and general peritonitis with adhesion of the intestines will probably set in, and in some cases there may be perforation of the intestines and escape of the freces into the peritoneal cavity. Death in such cases is almost invariable. 'The chief indications for treatment are the free administration of stimulants, and the local application of disinfectant lotions.

\section{Omphalorrhagia, hamorrhage from the umbilicus}

This may arise from inefficient ligature of the cord, from loosening of the ligature, or from ulceration or gangrenous destruction of the umbilicus or of the clots which close the arteries; or it may take the form of the so-called parenchy:matous or idiopathic omphalorrhagia, oozing drop by drop from the cavity of the umbilicus, a variety which is staunched with very great difficulty. Buhl has asserted, and it has been confirmed by several recent observers, that this form of umbilical hæmorrhage is, in the majority of cases, an indication of acute fatty degeneration of the liver, and post-mortem examination has shown that in such cases hæmorrhages of various sizes are to be found in the general integument, the mucous and serous surfaces, and in the parenchymatous organs. Sometimes this hæmorrhage is seen as the first indication of the so-called hæmorrhagic diathesis or hæmophilia, and also in resorption of biliary matter, especially in ariest of development of the gallducts.

Treatment.-When the hæmorrhage comes from the umbilical arteries it may be arrested by the application of a fresh ligature, if the stump of the cord have not already separated, or by the application of nitrate of silver; but if it be due to deep ulcera- 
tion or gangrene its arrest will be a more difficult matter. The parenchymatous hæmorrhage seems in general experience to have defied the most energetic treatment, such as the application of the perchloride of iron, the actual cautery, \&c. Dubois has recommended a form of acupressure in which two needles are inserted deeply into the tissues in the form of a cross, and the whole ligatured en masse. Hill has narrated a successful case where he arrested the hæmorrhage by pouring liquid plaster of Paris into the cavity, and keeping it filled with it.

\section{Umbilical hernia}

This is not to be confounded with the hernia into the substance of the cord (Nabelschnurbruch) already described, for it consists in the protrusion of intestine through the omphalic ring after the umbilicus has cicatrised. The sac may vary from the size of a hazel-nut to an apple, be roundish or conical, and its contents are generally small intestine, seldom omentum. It is due to an unusually slight power of resistance in the omphalic ring, and its rupture in some such exertion as crying. I have noticed in several families an apparent hereditary tendency to it. The bowel very rarely becomes incarcerated, and the hernia generally disappears spontaneously, but will be cured more readily with the assistance of an appropriate and wellfitting belt, the best consisting of a compress of cork covered with lint, fastened to an ordinary binder. 


\section{SEVENTH DIVISION}

\section{GENERAL DISEASES OF NUTRITION}

\section{Rachitis ; Rickets; Englische Krankheit; Osteomalaciu}

THis is a very wide-spread disease, for of 10,000 sick children in the Prague Hospital, 864 have been cases of rickets. It is a disease occurring exclusively in infancy, and expressing itself by disturbance of the growth and nutrition of the bones, by which they are exceptionally rich in water, fat, and carbonic acid, but unusually deficient in salts of lime, the proportion of these sinking from 63 per cent., their ratio in normal bone, to about 20 per cent., whilst the watery constituents may be increased from 30 to 100 per cent.

The gelatinous basis of the bones remains unaltered, but their specific gravity becomes lighter; they are softer, more elastic, and more easily bent or broken.

According to the microscopic investigations of Kölliker, Virchow, and $\mathbf{H}$. Müller, the changes consist of a remarkable extension of the spongy layer, an abundant proliferation of the cartilage cells in the primary cartilage layer, in deficiency or complete absence of calcification of the osseous and cartilaginous substance, in disproportionate extension of the medullary cavity, and delayed alteration of the cartilage tissue. A similar proliferation and thickening as in the cartilage are to be found in the periosteum, under which lies, instead of compact tissue, a loose but very vascular layer.

The lesions of the bones are usually preceded by certain disturbances of the general health, which may aptly be termed prodromata. These include dyspepsia, gastric, intestinal, or bronchial catarrh, restlessness, irritability, or actual expression of pain on being moved, softening of the cushions of fat, muscular weakness, emaciation, blanching of the skin, increased secretion of 
the phosphatic salts in the urine, and they may last for some time before the rachitic symptoms appear. In some exceptional cases the above-mentioned premonitory symptoms may not appear, and the bone affections may set in without any preliminary illness.

The rachitic alterations in bones affect their form, composition, and texture. The growth in length of the whole body is interrupted, and rickety children remain of stunted growth; and the special divisions of the skeleton are peculiarly affected as follows :

Skull.- This may have increased in actual size or only appear to have done so from the stunting of the body and the small size of the face; in form it is increased in its long diametcr (dolichocephalous), with the frontal and parietal prominences well marked; and it may be asymmetrical and scoliotic from unequal ossification and coincident hydrocephalus. The fontanelles are very slow in closing, sometimes remaining open as late as the fourth or sixth year, and the union of the sutures is very much retarded and very often marked by a saddle-like deepening along their course. In a certain class of cases the skull, especially in the occiput and parietal bones, is very easily indented, and feels parchment-like and pliable, and in the neighbourhood of the sutures may be felt interspersed insulations (Elsaesser's craniotabes); and if an opportunity be afforded of examining such a skull, these thin spots contrast markedly with the rest of the skull when it is held up to the light.

In them the diploic layer is very thin or has almost disappeared, while elsewhere in the skull, especially at the frontal bones, it will be found hypertrophied.

The teeth of the rickety child are long in appearing, due, undoubtedly, to the same process by which the bones of the skull are deprived of their necessary lime, the passage of the first tooth being delayed to the fourteenth or sixteenth month, or even later. I have had under my care a very markedly rachitic child, of four years old, in whom no tooth had appeared. Even when they do appear at the normal time they are small, deficient, and ragged at the edges from defective enamel, and they soon fall out.

Thorax.-Both the ribs and vertebre become affected to produce the characteristic appearances of the rickety chest. 
The cartilages of the ribs become enlarged, and form on both sides a row of symmetric nodules, which have been named the rachitic rosary. The thorax seeems relatively shortened, and the sides flattened in, or even indented, so as to resemble a saddle, its transverse diameter being in this way much shortened and the antero-posterior increased. The sternum may be arched forward, and the cartilages somewhat bent in behind it, constituting the "pigeon-breast" (pectus carinatum), and if one imagine a horizontal section of such a thorax, it would resemble a pear with the narrow end corresponding to the sternum.

In the vertebral column there may be numerous kyphoscoliotic curvatures, the most frequent and the earliest being the kyphosis of the last dorsal and tirst lumbar vertebræ, this being very frequently the result of the child walking prematurely, whilst by constant and improper carrying the child about on one arm scoliosis will be especially favoured. If we except caries of the vertebræ, the great majority of cases of spinal curvature during the first four years of life have their origin in rickets. The scapula is generally clumsy and misshapen, the clavicle often angularly bent and thickened, and the ribs sometimes infracted; and these collective changes give to the rachitic thorax, especially when well marked, an asymmetrical and distorted form very different from its normal contour; they also diminish the cavity of the thorax, and the respiratory movements are altered so that the lower lateral walls of the thorax sink in during respiration in a manner peculiar to the rickety thorax (Flankenschlagen rachitischer Kinder).

Pelvis.-The rachitic deformities of the bone are only of great importance when in after life they constitute an obstruction to labour. They are due partly to abnormal and asymmetrical growth of the periosteum and cartilage, especially on the edges of the pelvic bones, and partly to delayed and deficient ossification, the results of the latter reaching their height after the child has begun to walk.

Extremities.-Here the rickety condition is evident in enlargements of the epiphyses of the wrist and ankle, and, in extreme cases, of the phalangeal joints of the fingers and toes; also in curvature of the lower extremities, as seen in bandy and cross-leg, and in angular curvature of the thigh and arm bones, as the result of infraction. These latter changes may be caused 
by muscular action, or by the effort to sustain the weight of the body.

These rickety conditions of the skeleton, besides directly affecting the bones, produce indirect results in the organs to which the bones are related. Thus the rickety skull favours, by its soft, yielding structure and the late closure of the fontanelles, the occurrence of hyperæmia, hypertrophy of, and serous exudation into the brain, so that hydrocephalus, to a more or less marked degree, is a very common accompaniment of rickets. By reason of the softened occiput the contents of the skull may be exposed to pressure, which, exerted chiefly on the cerebellum and medulla oblongata, may give rise to spasm of the glottis or other nervous symptoms.

Rickety children often have a precocious mental development, and are restless, irritable, and easily excited. Sometimes the first symptom of rickets affecting the head appears in the form of profuse sweating of the scalp, and, according to my own experience, one symptom which is never absent is a rubbing and pushing the head into the pillow, by which the hair is worn off.

A rickety state of the thorax, in combination with a weak state of the respiratory muscles, produces and maintains frequent and obstinate bronchial catarrh, broncho-pneumonia, atelectasis, shortness of breathing, asthmatic attacks, hypertrophy and cheesy degeneration of the bronchial glands, and cyanosis.

The abdomen is usually distended (Froschbauch-frog-belly), and presents a marked contrast to the short, narrow chest, and the thin, weak extremities.

The spleen is almost always hard and enlarged, sometimes to as much as three or four times its normal size; and when the disease is severe, it, together with the liver and kidneys, may also be found to have undergone anyloid degeneration. Intestinal catarrh is frequently associated with rickets.

Rickety children are long in beginning to walk, their gait being uncertain and waddling, and they are easily tired. I have seen a case in which walking was impossible until the sixth year.

The peculiar pains in the bones affected afford a very persistent sign of the disease, explained by Trousseau as due to the 
hyperæmia of the periosteum, though this is far from being certain.

The head-sweats already mentioned, and the tendency to general perspiration, often produce copious crops of sudaminal eruption.

The disease may take two courses, either the acute or chronic, the former being very rare, the latter presenting the usual type of the disease and lasting over some years, or at least some months. I have seen the disease in a well-marked acute form only ten times out of several thousand rickety children. It usually begins between the fourth and ninth months in a child which seemed previously in good health, and who may be either at the breast or have been lately weaned. The initial symptoms consist of loss of appetite, loss of and restlessness during sleep, peevish crying while awake, some degree of fever, vomiting and diarrhœa followed by very rapid emaciation, disinclination to sit or walk, with a decided preference for lying, and marked indications of suffering when lifted or touched. At the same time periosteal swellings may be found in the limbs, especially on the thighs, which may give rise to a suspicion of some injury; and after these symptoms have lasted for two or three weeks they disappear as rapidly as they came; the sleep and appetite soon become normal, the pain disappears from the limbs, and the child can go about or be moved without discomfort.

I have seen acute rickets twice fatal in children affected by congenital syphilis.

Chronic rickets may begin six or eight weeks after birth, or about the time of the first dentition, and it usually ends about the fourth or fifth year, seldom later ; while its effects, especially on the skeleton, usually continue throughout life. Of 846 accurately investigated cases, as they came under treatment, 346 were not a year old, 368 above a year, 112 two years, 24 three years, and only 14 were four years old. Rachitic lesions do not always follow a definite order of occurrence, as Guerin has stated they do, in the direction from below upwards; buit they may follow various orders, beginning to be manifested sometimes in the skull first.

As. recovery sets in the growth of the bones becomes more satisfactory; the sutures and the fontanelles close, and the teeth 
appear, the periosteal enlargements disappear, the appearance of the child becomes healthy and strong, the muscular weakness disappears, the digestion improves, and finally its power of walking is restored. The child usually remains somewhat undersized, but the disproportionate relative sizes of different parts of the body disappear, and the bones assume their normal, hard, and compact character.

Causes.-Occasionally rickets may be found to be congenital, a fact well illustrated by an interesting preparation of a rickety fœtus in the Museum of our Hospital. Of the immediate cause of rickets we are yet ignorant; but that there are definite predisposing and favouring influences for its production we are quite certain. Thus its hereditary tendency is very well marked, for we often find that the parents of rickety children have been rickety in their youth, or in after life they may have evinced tendencies to some of the allied pathological conditions of tubercle or syphilis, or have suffered from marasmus or anæmia, this being especially the case on the mother's side. After the hereditary disposition the most important factors in the production of rickets are unsuitable food and residence in cold, damp and badly ventilated dwellings, and a want of attention to cleanliness. The disease, hoivever, is not exclusively found amongst the poor, and may be met with amongst the richest and best cared for, though the cases then have a less severe course. In my own experience male children scem to suffer more than female, for of 864 cases, 500 belonged to the former sex and 364 to the latter, though the observations of others seem to show contrary results.

Complete recovery, with partial or even perfect disappearance of all the local results of the disease, frequently takes place, though rickets may prove extremely dangerous to life either directly or indirectly, or it may even have a fatal issue. Any pulmonary or cerebral complication is very much to be dreaded in rickety children, as also are laryngeal spasms, general convulsions, chronic follicular enteritis, or severe anæmia, for they seem to have but a very slight capacity for resisting the effects of almost any acute disease. The yuunger the child and the more marked the symptoms, the more unfavorable must be any prognosis.

Treatment.-This may be divided into causal and symp. 
tomatic, and both must be dietetic and pharmaceutic. To the first division belongs the prophylactic treatment of the parents, especially of the mother during gestation. If the mother be anæmic, and appear to have suffered in early life from rickets or scrofula, she must be subjected to careful dietetic regimen, and to a course of quinine and iron. If the mother's health is bad after her confinement it will be safer to find a nurse for the child; or if one suitable cannot be found, it must be reared on some substitutes for its natural nourishment, such as have already been treated of. If the child be already weaned when the disease appears, then specially nourishing food of easy digestion, such as strong beef tea and pounded raw meat, must be given, combined with the administration of some stimulant, such as beer or wine. Removal to fresh air and better dwelling place, especially to forest air in summer, and minute attention to cleanliness are also essential. Rickety children should not be laid on feather beds, but should have horse-hair or sea-weed mattresses.

Of pharmaceutic remedies cod-liver oil and preparations of iron are the most serviceable, the former being given in doses of twenty or thirty drops to children, and continued for a long time, if it does not produce vomiting or diarrhœa. The lactate, pomate, or saccharated carbonate of iron, phosphate of iron and soda, and the compound syrup of the phosphates, are the best preparations. If the digestion be weak the iron may be combined with quinine or with small doses of rhubarb.

The symptomatic treatment includes, of course, the consideration of numberless possible complications which are treated of under their proper divisions. To alleviate the general restlessness of the children, I am in the habit of advising them to be bathed frequently, at first with lukewarm and afterwards with cold water; and for the relief of the pain in the bones the use of some sedative liniment or compresses soaked in olive oil in combination with chloroform, oil of hyoscyamus, \&c. Occasional baths at a temperature between $37^{\circ}$ and $40^{\circ}$ are useful, especially with the addition of extract of malt, or extract of pine wood or pine leaves, hops, aromatic herbs, the Frangenbad salts, or the ordinary sea salt; and for the muscular weakness the rubbing of the child with some aromatic spirit lotion is to be recommended. 
Great care should be taken that the child is not allowed to get upright, to stand, or to walk, for by preventing it doing so many curvatures may be avoided. When these do become established, they may be counteracted, or at least very much improved, by proper orthopædic treatment, and this should always be adopted before the bones become again consolidated, especially in the lower limbs, for after they have become set again it is impossible to remedy the deformities, except by osteotomy or by Billroth's plan of using a hammer and chisel, and the subsequent application of a plaster-of-Paris bandage.

The treatment of scoliosis and deformities of the thorax is much more difficult, for as long as the rachitic process is going on, not only are all apparatuses, whatever their construction, useless, but they are positively hurtful by interference with respiration, and I content myself with keeping the patient confined to a hair mattress until reconsolidation of the bones has begun, and after that some apparatus may be found useful.

\section{Scrofula}

This dyscrasy is almost peculiar to infancy and youth, and is characterised by hyperplasia and very obstinate recurrent inflammations of certain tissues, which are excited by very slight causes, and which in healthy people would pass off without damage. The most common seat of scrofulous indications is the lymphatic system, and perhaps this may be exclusively its seat of origin by some diversion of course or of character in the lymph-stream, local at first and becoming general in sequence. Scrofulous diseases are very common, for among 10,000 patients we have had 1192 cases of scrofula.

Of the pathological conditions of scrofula there are many varieties, such as simple cellular increase (hyperplasia), inflammation, suppurative softening, ulceration, molecular disintegration (cheesy degeneration-Verkäsung), and induration; and coincidently we frequently find indication of tubercle, the connection of which with our present dyscrasy we shall afterwards consider.

Scrofula does not evince itself essentially in the formation of cheesy centres, and the scrofulous diathesis and the disposition to cheesy degeneration are by no means identical, though it 
cannot be denied that the scrofulous diathesis shows a great tendency to tyrosis of inflammatory products.

The scrofulous habit, by which term is meant the sum of several distinctly marked external localisations of this disease, will often come under notice, though not with unfailing regularity. As the result of many observations, a distinct variety, which may be termed the torpid scrofulous habit, has been defined, marked by a pasty, puffy and somewhat plump body, with a large proportion of fat, pouting lips, thick pear-shaped nose, waxy white skin, deficient bodily strength and dull mental powers. As a very marked contrast to this there has been described an erethetic habit marked by a fine gracile figure, slight delicate features, bright eyes with blue sclerotic and long eyelashes, fair hair, fine white and transparent skin, weak muscular system, and great mental irritability ; and even if these two different forms cannot be allowed to have an absolute value, yet for clinical purposes their usefulness cannot be denied.

Scrofulous changes possess no histological elements or processes proper to them, but have only certain peculiarities, such as the multiple points of appearance and their insidiously chronic course, the frequency of relapses, and their obstinate resistance of remedies, which stamp the condition as a special diathesis.

Local scrofulous diseases show themselves most frequently in the lymphatic glands, the skin, mucous membrane, bones, and joints, and they have, if they occur in isolation, an origin apparently of an entirely local character, excited by some ordinary morbid influence, until in their further development they take on the peculiar scrofulous action.

The following statement of the frequency of various local affections of a scrofulous character is arranged from an analysis of 1192 cases.

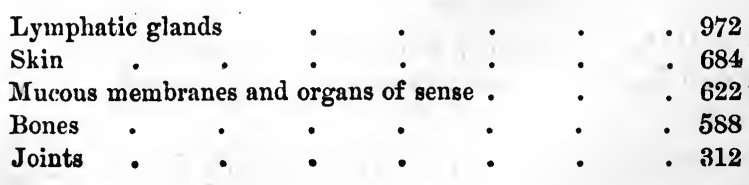




\section{Lymphatic glands}

In many cases the glandular affection is secondary, arising from the condition of the lymphatic vessels of the skin, mucous membrane, bones, and joints; though it may also be an independent condition, not arising from any centre, but due solely to the scrofulous diathesis. Its anatomical character may vary in that the enlargement may be due simply to increase of the lymph-cells or to their accumulation in the follicles (hyperplasia); or it may be of an inflammatory nature, either acute or chronic, the latter being far the more common, and ending either in resorption, partial or.complete suppuration, cheesy disintegration (tyrosis), amyloid change, fibrous hypcrtrophy (induration), or, least common of all, sarcomatous degeneration. The swollen and inflamed glands may vary in size from a pea to a hen's egg, and are often found in a chain like a string of beads, smooth, easily movable, and painless. Sometimes, however, they are painful, and the surrounding tissue infiltrated with inflammatory exudation which readily breaks down, and along with coincident action in the gland produces abscesses, sinuses and sluggish ulcers, which give out a thin purulent fluid and present undermined walls and bridges of skin stretched across the cavity. When they heal their cicatrices are much corrugated. These ulcers sometimes become gangrenous in hospitals, and under circumstances where they are not carefully attended to.

Schüppel has arrived at very different conclusions, by which he narrows the province of scrofula very materially. He regards the cheesy degeneration of lymphatic glands as belonging much less to scrofula than to tubercle. He believes the two conditions to be identical, and only admits a pure scrofula as a stage in which patients affected by the tubercular diathesis are found before they become tubercular; and scrofulous conditions of the skin, mucous membranes, and superficial lymphatics, only the natural result of a tubercular eruption. With these views my own clinical experience does not completely harmonise.

Broncho-adenitis and tabes meseraica may here be alluded to as the results of the implication of internal glands; and a general glandular affection of long duration may lead to leucocytosis. 
The most frequent seats of enlarged glands are the neck, groin, axilla, the hilum of the lung, the anterior mediastinum and the mesentery.

Care must be taken not to set all glandular enlargements met with in children down to a scrofulous origin, for they may be found in the cervical, occipital and auricular regions as the result of dentition, stomatitis, angina, and skin eruptions, without any reference to a scrofulous taint.

\section{Skin and subcutaneous cellular tissue}

The skin is a very frequent seat of scrofulous diseases, the varieties of chronic dermatitis of a scrofulous character being numerous, their favourite seats being the hairy scalp, the face, behind the ears, the nose and eyes, the flexures of the joints and the genitals. Eczema is the most common kind, and after that impetigo. The coarser papular and pustular eruptions, such as ecthyma, rupia, and pemphigus, are less common. Lichenous eruptions are seen on the extremities, and less frequently on the trunk. Ulcerative follicular dermatitis and lupus occurs chiefly in the nose, less frequently in the cheeks, lips, fingers and generally over the surface, leading, in some extreme cases, to extensive destruction of organs. Occasionally we see the acute forms of skin disease, such as fixed or fugitive erysipelas and urticaria, induced by the presence of chronic suppurating centres, and under the influence of such attacks chronic skin eruptions and ophthalmias will often rapidly disappear.

Scrofulous skin diseases usually are developed between the first and third years of life, though the ordinary scald-head and milk-thrush are often met with in sucklings.

Scrofulous inflammation of the subcutaneous cellular tissue results in abscesses varying in size from a hazel-nut to a pigeon's egrg, such as used to be described as scrofulophymata. They occur chiefly in the soft tissues of the face, the buttocks, backs of the hands, and feet; and they sometimes disappear, after lasting many months, by resorption, without the discharge of their contents. The more frequent result, however, is the discharge of a quantity of flocky, thin, unhealthy looking pus, the contents of the so-called cold abscess. Large abscesses of 
this kind are sometimes developed between the muscles of the extremities, and they are excessively difficult to heal.

We often see the cellular tissue of the lips and nose, and, in girls, of the labia, thickened by infiltration of a serous character.

\section{Mucous membranes and organs of sense}

The most common scrofulous diseases of these structures are of a catarrhal and inflammatory nature. Thus in the eye we may have chronic inflammation of the meibomian glands (blepharoadenitis), hordeola with destruction of the eyelashes, thickening of the edges of the lids (tylosis), conjunctivitis, formation of pustules on the conjunctiva and sclerotic, accompanied with the formation of bands of vessels, and subsequent formation of opacities (maculæ corneæ), parenchymatous keratitis and ulceration, synechia and staphyloma-all the result of the scrofulous taint and invariably characterised by great photophobia and lachrymation. The photophobia is sometimes very persistent, lasting for months, and compelling the poor child to keep his face buried in the pillows, and thus exciting erythema and excoriations round the eyelids.

The mucous membrane of the nostrils is also sometimes affected by chronic scrofulous inflammation, which produces an abundant sero-purulent secretion, with reddening and excoriation of the nostrils; and this disease, from its chronic course, may establish an ozæna, with its results of necrosis of the turbinated bones, perforation of the septum and palate, and even the occurrence of purulent meningitis.

Inflammation of the ears in scrofulous children is generally external and simply catarrhal, accompanied by a muco-purulent discharge and swelling, dryness and reddening of the tympanum, though in very severe cases the membrane may be perforated and the inflammation extend inwards. It may be phlegmonous and result in a boil which may reach the size of a bean.

Internal otitis may exist without any affection of the external organs, and may be either simply catarrhal or due to periostitis, with destructive relaxation or perforation of the tympanum, evacuation of the ossicles and loss of the sense of hearing of a permanent, or it may be transient, character, and sometimes 
also with paralysis of the facial nerve. Occasionally we have even graver complications, in the form of necrosis of the mastoid process and dislocation of the auricula forwards, thrombosis of the cerebral sinuses, tubercular or purulent meningitis, encephalitis, and abscess in the cerebellum.

Pharyngeal catarrh with hypertrophy of the tonsils frequently occurs in scrofulous children, and we have other mucous membranes, such as those of the air-passages, the intestinal tract, and the vagina, also suffering from chronic inflammation of a very obstinate and remitting character. In scrofulo-tubercular children we often find ulceration of the intestinal mucous membrane; but whether this belongs to the scrofulous or tubercular diathesis, or to a mixture of the two, cannot yet be determined.

\section{Bones}

Scrofulous diseases of the bones are very abundant, and may originate in periostitis, in osteitis, or in osteo-myelitis ; here also, as elsewhere, taking the various forms of hyperplasia, inflammation, suppuration, and destruction or death of the affected forms, caries or necrosis; the former affecting the articular extremities and epiphyses more particularly, and the latter the shafts. The wrist- and ankle-joints, the metacarpal and metatarsal bones, and the phalanges of the feet and hands, are very frequent sites of scrofulous inflammation. A somewhat rare form of chronic osteitis or osteomyelitis, of a very characteristically scrofulous character, is to be seen in the so-called spina ventosa. It is due to the formation of abscess in bones, most frequently the proximal phalanges of the fingers, which present round, spindle-shaped enlargement, with cloacæ here and there; the progress of the disease being accompanied by severe pain. Periosteal abscess of the sternum and ribs, followed by necrosis, is also a somewhat infrequent form of bone disease. In the skull we find the petrous bone, the zygoma, the lower jaw, and sometimes the calvarium affected. In the latter case I have seen necrosis of a large sequestrum followed by prolapse of the brain and fatal purulent meningitis.

Disease of the spine has an unfortunate notoriety in connection with the scrofulous diathesis, occurring as spondylitis and spondylarthrocacia. Its most common seat is in the dorsal 
regrion of the spine, less frequently in the cervical and lumbar, and very rarely in the sacrum. The inflammatory and suppurative processes are rarely limited to their point of origin, but lead to a perforating abscess, which points, according to the region in which it occurs, in the pharynx, back, groin, or thigh.

By destruction of the bodies of the vertebræ angular curvature is produced, and sometimes accompanying deformity of the thorax. In its initial stage this disease is not always to be easily recognised, but it is generally marked by severe local pain with nocturnal exacerbations, fever, loss of appetite, restless sleep, wasting, weariness on the slightest exertion, zonular pain in the affected region, and severe pain in moving the head or limbs. The extension of the inflammatory action to the meninges or to the marrow itself is likely sto lead to paresis, or paralysis of the limbs, bladder, and rectum. As in all scrofulous diseases, such a disturbance of the general health by hectic, \&c., is apt to supervene as to destroy life. Spinal disease usually sets in spontaneously, though it cannot be denied that it may be excited by injuries in those who suffer from the scrofulous taint.

\section{Joints}

The joints are frequently affected by scrofulous disease, which may either be simple purulent inflammation or a fungous inflammation known as tumor albus. These originate more frequently from the synovial membranes than from the articular surfaces, but sometimes from both.

The progress of such cases is marked by suppuration and caries of the articular elements, effusion of pus into the periarticular connective tissue, the formation of abscesses and their pointing outwards, the formation of fistulous tracks, and the. occurrence of subluxation and luxation of the bones of the affected joints. When a natural cure results the destruction is imperfectly repaired by a process of granulation, and the disorganised joint becomes anchylosed.

Such diseases may be either acute or chronic; their duration and symptoms are very various and their results very often unfortunate, for the subjects of them have to run the terrible gaumtlets of pyæmia, Bright's disease and tuberculosis.

In hip-joint disease (coxitis, coxalgia), the most frequent of 
these scrofulous joint diseases, the earliest and most important symptom is pain, felt at first in the knee and later in the hip, and increased on any movement, though in some cases it is entirely absent. A slight lameness or dragging of the affected limb is all that can sometimes be found as an indication of the early stage of this insidious disease. In the later stages the patient suffers from fever and hectic, becomes emaciated and pinched, and is unable to move about without excruciating pain. The position of the limb becomes very characteristic, and as the disease advances so does that of the pelvis and vertebral column; the buttock of the affected limb becomes flattened and its folds obliterated, the thigh is more frequently abducted than adducted, and rotated slightly outwards, more rarely turned inwards, and it is drawn up towards the abdomen. The pelvis is bent towards the affected side, and is so tilted in order to give relief that the diseased limb will appear the longer of the two, even when softening and absorption of the head of the bone has produced absolute shortening.

In inflammation of the knee-joint (gonitis, tumor albus genu) the symptoms are more or less severe as the disease takes the purulent form or that of fungous proliferation. The joint undergoes change in shape, severe pain is felt in it, and its function is destroyed; and as the disease progresses the joint becomes destroyed and subluxation of the bones occurs.

Similarly scrofulous disease may affect the phalangeal, iwrist, elbow- and shoulder-joints.

\section{General course and result of scrofulosis}

It may be laid down as a rule that the course of all scrofulous diseases is insidious and extremely chronic, and that the more numerous the localisations of the disease are in any particular case, the more the general condition of the patient will be affected, and the more readily mischief in old centres will be lit up, and new centres of disease excited. In the majority of cases scrofulosis ends in recovery if the child's circumstances are favorable, and the proper treatment be carried out ; and this recovery may be perfect, or it may be only partial, a certain delicacy of constitution being left behind; and recovery is always more rapid and complete if the disease has been limited 
to some external organ, such as the skin, some organ of sense, or the superficial lymphatic glands; and, on the contrary, less certain and more prolonged when the disease is internal.

What is most and always to be feared is tubercle, the relation of which with scrofula, in spite of the extremely valuable experimental investigations of Buhl, Waldenburg, Cohnheinl, and others, is still far from being definitely determined. Some of these authors place scrofula and tubercle in the relation of cause and effect; in other words, that tubercle is due to a scrofulous origin by infection, by the resorption of the cheesy and purulent matter, a statement which can have only a relative, but scarcely any general value. Whoever has had the opportunity of examining large numbers of cases of scrofula will be driven to the conclusion that scrofula and tubercle, if at least not identical, are diseases which are very closely related in their causes, in their pathology, and in their clinical history; and that though they often occur coincidently or in sequence, it is not a matter of necessity that they should do so. If the embolic process of infection were the sole connection between these two diseases, my impression is that tubercle would be much more common in scrofulous children than is the case. I may allude, for instance, to the extensive and deep ulcerations, and the sometimes numerous abscesses seen in the course of spinal caries with which tubercle is seen very rarely in association, though there exists in those cases every facility for the processes of infection; whilst, on the other hand, I have seen tubercular meningitis in cases where $I$ have been unable to discover the slightest trace of any cheesy or purulent centre in any other part of the body. 'The theory that there may have been an earlier centre of infection which has disappeared is too unsubstantial, and could only be verified by a previous examination. Schüppel even states the resorption theory as if contrary to his conviction, and very justly says that we might fairly expect that if once the matter which excites the tubercle gain admission to the blood, there should be a general miliary tuberculosis, or at least the presence of tubercle in all those organs in which it chiefly occurs. The peculiar immunity from tubercle which entire organs and systems of tissue enjoy will, no doubt, be explained by the peculiarities of their ultimate structure. It is, further, a curious fact that in cases of extensive 
tubercle, where the material which produces it must exist very extensively in the blood, we have the same organ sometimes affected and sometimes entirely free; and also that a child with cheesy degeneration of the glands of the neck will be found to have tubercular meningitis, whilst all the other organs in which tubercle is usually found may be entirely free from it. Those who accept the resorption theory must, therefore, look for subsidiary causes in the conditions of the organs affected to explain the diffusion of the tubercle. We, on the contrary, are inclined to regard the diathesis of the individual as the predisposing, and the irritative condition of the glands as the proximate or exciting cause of the tubercle.

Scrofulous children are exposed to other dangers, such as amyloid degeneration of the abdominal glands, the liver, spleen, and kidneys, the affection of the latter being especially prejudicial to life. The production of such lesions is especially due to the influence of prolonged suppuration from diseases of bone.

Pyæmia and hectic fever are also common causes of death in scrofulous children.

Causes.-Scrofula affects children of all ages except those at the breast, although even in them obstinate nasal and bronchial catarrh and eczematous eruptions sometimes seem to betray the beginning of the disease. The sexes seem to be pretty equally affected, for of 1192 cases, 584 were boys; and 608 were girls, and the largest number of cases occurred in the months of April, May, March, February, June, and September; the fewest being met with in December, November, October, and January. The largest proportion of cases, especially of those occurring under favorable hygienic circumstances, is that in which the disease, or rather a tendency to it, has been inherited from scrofulous, tubercular, or syphilitic parents, especially if they be closely related. Next in importance to the hereditary disposition may be mentioned as a cause of scrofula errors of diet, especially in children who have been artificially reared by use of a preponderance of starchy food, such as bread, rusks, potatoes, and pulse, and also the residence of such children in an impure air and in damp, newly built houses.

Scrofula is especially, through not exclusively, a disease of the poor in great towns and manufacturing centres, but it may also 
be seen, under the circumstances above alluded to, in the families of the wealthiest. There are certain occasional causes which seem to excite the latent tendency to an active existence, and amongst these may be mentioned vaccination, exposure to cold, measles, hooping-cough, and other acute diseases, after the occurrence of which scrofula seems to appear as a sequela.

Diagnosis. - When the disease is well marked by multiple localisations, the diagnosis presents no difficulty; but in the early stage, and with few local indications, it may require much care and caution.

The present condition and past history both of the child and its parents must be considered, as must also the surrounding relations and the course of the disease, the various scrofulous lesions having of themselves, as already remarked, no specific pathological or clinical characters.

Trealment.-In this our efforts must in great measure be directed to the improvement of the constitution by a careful attention to diet, and to the local treatment of the disease. The children of tubercular, scrofulous, and weakly mothers must be provided with a nurse, and may be kept at the breast till they are ten or twelve months old. Children who are somewhat more advanced than this should have a carefully selected diet of mixed animal and vegetable food, the recent plan of feeding them entirely on animal food, as is recommended by some practitioners, seeming to me unsound.

The food should consist of strong soup, milk, white flesh, eggs, fresh vegetables, and fruit. For drink young children should have milk, coffees, especially acorn coffee, and the more advanced should have beer and diluted wine. Removal to fresh country air, especially to some sheltered forest district, or to the sea-side, and the use of moderate exercise and bathing, will be found useful in expediting recovery.

Of drugs, the most trustworthy are cod-liver oil and a combination of iron and iodine, such as the syrup of the iodide of iron, the former being administered in doses of one or two teaspoonfuls to young children, unless it be found to interfere with digestion, and a mouthful of black coffec or chocolate taken immediately after will be found to relieve the disagreeable taste. For any gond to accrue from the use of the oil, or of any of the other remedies in the treatment of scrofula, they must 
be persevered in for a long time, for months or even for years, though in summer the oil may often be dispensed with, and some chalybeate water containing iodine or bromine-such as Hall, Kreuznach, Krankenheit, or Adelheidsquelle-substituted for the drugs. But in any kind of treatment a careful watch must be exercised, so that the digestion be not disturbed.

In very anæmic and weakly children I generally recommend some mild chalybeate, such as the lactate, saccharate, or pyrophosphate of iron, to be given to the child before the iodide of iron is begun. A very common popular remedy amongst poor people consists of walnut-leaves, given both as infusion and in their fresh state, also externally in baths. Mineral water and sea.baths will often be found serviceable, and also those containing extract of pine.

Local treatment.-Enlarged glands may be treated by the external application of tincture of iodine, or by its injection into the glandular tissue; and in cases where they are isolated, compression may be used for the purpose of reducing them. Their removal by operation has also been advised. When there is a tendency to suppuration some emollient plaster should be applied, or poultices should be used, and care should be taken as far as possible to obviate a scar in any place where it may be visible, especially in girls. ${ }^{1}$. Ulcers should be treated by great attention to cleanliness, the application of some mild mercurial ointment, occasional touching with caustic, and on the appearance of any tendency to necrosis the liberal use of detergent lotions, such as solutions of chloride of lime or carbolic acid.

In scrofulous inflammation of the eyes, light and air must not be too rigidly excluded from the patient, and in cases where the disease appears as pustular conjunctivitis and keratitis the best remedy will be found to be dry calomel applied by a hair pencil. When the cornea is attacked by parenchymatous and ulcerative'inflammation, the most soothing applications will be found to be laudanum, solution of atropine, and blue ointment containing some atropine or extract of belladonna applied round

1 For this end $I$ have advised the methodical tapping of glandular abscess in the neck by a hypodermic syringe, as often as necessary, and as long as they secrete pus.-L. T. 
the lids. Blisters on the temples or behind the ears are also useful.

To relieve the distressing photophobia the local application of belladonna and atropia, and some opium internally, are the best, and in some cases I have used with advantage the rather heroic remedy of bathing the child's head abundantly with ice-cold water. For chronic blepharitis the red and white precipitate oitments are invaluable, and turbidity of the cornea will often yield to the application of dry calomel.

In otitis, cleanliness must be most carefully attended to by injections of luke-warm water or infusion of marshmallow, and after their use some astringent may be applied, such as alum or sulphate of zinc, or some disinfectant if the discharge be offensive. Thickening of the tympanum and its consequent impairment of hearing may be relieved by diligent rubbing of iodine ointment behind the ear.

Scrofulous diseases of the skin are very inveterate and troublesome. In cases of eczema rubrum a lotion of neutral acetate of lead (Goulard's solution) should be applied until the skin is whitened; in eczema squamosum a weak pitch ointment will be found more serviceable; and in cases when the incrustation is very troublesome, the white precipitate ointment may be tried alone, with caution, or in combination with bismuth ointment. Eczema in the cutaneous folds, as the groin, genitals, and axilla, is best treated by some dry powder, as starch or oxide of zinc. In obstinate eczema of the extremities, Hebra's plan of enveloping them in plaster is a good one, as it prevents the increase of the disease by the child's scratching.

For lichen and dry eczema the red precipitate ointment, Dutch soap, sulphur ointment, and Vlemingkx's solution will be found the best applications. The milder forms of lupus may yield to mercurial ointment and tincture of iodine, but if they do not, the ulcers must be destroyed by some active caustic.

Cold abscesses may be treated by injection of tincture of iodine and compression.

For the affections of bones and joints some special surgical treatise had better be consulted; but generally, it may be said, especially for coxitis, that absolute rest is necessary, to be secured by the adaptation to the affected joint of some immobile apparatus. Pain may often be relieved by cold-water packing. 
Reposition in case of luxation may be done under chloroform. When suppurative destruction of a joint has set in, then some surgical proceeding, such as resection or amputation, may have to be considered, and this should not be delayed until pulmonary tubercle has become deposited.

Inflammation and caries of the vertebræ demand absolute confinement to the horizontal position, and the general treatment of symptoms as they arise.

\section{Tuberculosis}

Under this term is included any lesion of nutrition which results in the formation of numerous small grey nodules known as tubercle, and which, according to some authors, have an inflammatory origin. This tubercle consists, in its early stage, of an accumulation of transparent, delicate cells, for the most part nucleated, and according to the results of the majority of observers they take their origin from the walls of the arteriocapillary vessels. As the tubercle grows older it degenerates into a cheesy mass from the destruction of the cells by fatty degeneration, and its grey colour passes into a yellow.

Schüppel, in his valuable monograph, 'Untersuchungen über Lymphdrüsentuberculose,' 1871, has advanced opinions regarding the nature and origin of tubercle which differ very materially from those in general acceptance. He describes glandular tubercle as consisting of roundish and sharply defined nodules, not always perceptible to the naked eye, and at the most of a diameter 0.3 millimètre. They are always situated in the vascular follicles of the glands, no matter what position or form these follicles possess. The tubercle formation begins in the track of the blood-vessels, and consists not merely of a collection of small round cells, but of a sharpiy defined though small mass, destitute of blood-vessels, but consisting of variously sized cells, some reaching to a considerable size, and a reticulum, in the meshes of which the cells are imbedded. In the tubercle of lymphatic glands three different cell-forms are to be met with-giant cells, large epithelial cells, and small cells resembling lymph-corpuscles. 'The first are peculiar to tubercle, and their presence in a lymphatic follicle is always indicative of the tubercular change; an association which does not exist with 
the formation of new connective tissue, the result of a previous inflammatory attack. Sometimes in the immediate surrounding of tubercle there is a proliferation of the connective tissue, and in the centre of this there may be cheesy detritus, whilst the cortex consists of fibrous tissue, forming nodules to which the names of fibrous and hard tubercle have been applied. Schüppel classes the cheesy condition which is so often found in hyperplastic glands with tubercle, though it is usually regarded as belonging to the second stage of scrofula.

The tubercular nodules are sometimes situated apart or in groups, or they may run together in a mass and form a large yellow knot or a diffuse infiltration. During the course of its existence the tubercle may undergo various changes, as, for instance, the cell-detritus may become absorbed and the tubercle disappear with cicatricial contraction of its mother-tissue, or it may become calcified, though neither of these processes are of frequent occurrence in childhood; or finally, the destruction of the tubercle, followed by the destruction of the tissue in which it is deposited, may lead to the formation of cavities as in the lungs, or of ulcer's as on mucous membranes. The tissue surrounding the tubercle may remain unaltered or the local irritation may excite hyperæmia, or even fibrino-purulent inflammation, as tubercular meningitis or pneumonia. We often see the tubercular process extend gradually over neighbouring tissue, as, for instance, in children from the bronchial glands to the tissue of the lungs, though the reverse may also be the order of the infection. Such extensions are most probably due to the urgency of the lymphatics, as pointed out by Virchow and Buhl.

Tubercle may be of primary occurrence, and is then sometimes limited to certain organs, as the glands, the lungs, meninges, larynx, or urino-genital system, but sometimes it is met with simultaneously in several organs. It may also be secondary, this being more frequently the case, and it may then be met with in almost every organ in the body. In tubercular children other pathological conditions may be found, as hydrocephalus, acute and chronic intestinal catarrh, dysentery, and fatty and amyloid degeneration of the liver, spleen, and kidneys.

The following results have been established by Neureutter 
and myself from 302 dissections made in the Prague Hospital : - In 42 cases the tubercle was found in one organ only; in 48, in two organs; in 62 , in three; in 47 , in four; in 42 , in five; in 28 , in six ; in 20 , in seven ; in 6, in eight ; five times in nine, and twice in ten different organs.

We found it to affect the bronchial glands in 275 cases, the lungs in 175, and the stomach in 4 only.

The disease may be acute, the tubercle being then generally found in several organs, or it may be chronic and be limited to an organ or to two or three.

The symptoms of tubercular lesions vary with their seat, course, the number of the centres of the disease, and its complications. The various organs in which tubercle is more usually found have been previously referred to, the only others needful to add here being the stomach and intestinal canal. In the former I have found tubercle only eight times. Its cavity was generally found to be distended with gas, and on the mucous surface were some patches about the size of a threepenny piece, indicating loss of substance, varying in number and having the edges swollen, undermined, and irregularly indented. The bases of the ulcers were greyish-yellow or dusky red in colour, and the corresponding peritoneal surface was dark red and scattered with greyish nodules perceptible to the naked eye. The symptoms of this condition include frequently recurring pain over the stomach aggravated on pressure, frequent vomiting. with the ejected matter streaked with blood; and these symtoms will be especially trustworthy if there be found at the same time indications of tubercular lesions elsewhere.

Tabercular disease of the intestines is seldom a predominant lesion, but it occurs in nearly a third of all the children affected by tubercle, especially when the infection is general. It is met with, as in other organs, partly as greyish nodules, corresponding to the solitary glands and to Peyer's patches, and partly as tubercular infiltration affecting roundish spots of mucous membrane, which may be isolated or confluent, and which result in ulcers with hard thickened edges extending in the direction of the transverse axis of the gut. The most frequent seat is the small intestine, and least frequent the large gut. The mesenteric glands are constantly found enlarged, and having undergone cheesy or tubercular ch inge. 
The disturbance of digestion which attends this intestinal phthisis is in direct proportion to the amount of the ulceration.

There is almost always an obstinate, long-continuing and ever-recurring diarrhœa, which is especially troublesome during the early hours of the morning, and gets therefore the name of diarrhoe nocturna. It is characterised by pultaceous or watery stools of a dark brown, yellow, or greyish colour, resembling yeast, and these symptoms, with the coincidence of advancing emaciation and the presence of other tubercular deposits, may afford sufficient ground for a diagnosis. This, however, must be made with caution, as such symptoms may be due to simple. follicular ulceration.

Causes.-Tuberculosis has been found, as already mentioned to be congenital, but it is much more frequently developed after birth; its most common time of appearance being the second and third year, though I have found it in sucklings as early as eight weeks old. The sexes seem to suffer with equal frequency. The most important factor in its production seems to be the hereditary tendency, for parents who are scrofulous or tubercular, or whose health has materially suffered from exhausting diseases or excesses, are apt to beget children with a tubercular tendency. The disease may be developed independently by bad sanitary conditions, and also by the embolic impaction of purulent and cheesy matter, a fact which is now beyond all doubt.

Buhl regards miliary tubercle as the result of the existence of cheesy centres, especially in the lungs and lymphatic glands, and ranks it as a specific disease, the result of resorption and infection spreading the tubercular material through the lymph and blood, and their systems of vessels.

Waldenburg, Cohnheim, and Frankel accept the resorption theory, and go still farther in stating that tubercle originates in the admission of disintegrated and inspissated pus into the circulation. Klebs and Villemin regard tubcrcle as due to a specific infecting matter dissolved in the fluids of the body.

Niemeyer has arrived at the result that tuberculosis is generally a secondary disease, arising in some way, not yet very clear, from the influx of the products of cheesy degeneration, though he does not regard it as constantly and necessarily dependent on the pre-existence of cheesy centres.

What my own views are I have already explained when 
speaking of scrofulosis, and I share the opinion that tuberculosis is often a secondary disease, arising from cheesy deposits in some yet unexplained way; but also that this is not constant, for it may appear primarily as an expression of the tubercular diathesis in several situations simultaneously. The latter fact is especially the case in children in whom the disease is hereditary, and in my opinion sufficient importance has not been given to it.

Certain diseases, as measles, hooping-cough, pneumonia, pleuritic effusion, long-continued intestinal catarrh, seem to develope in many cases the latent tendency to tuberculosis in a more or less pronounced manner, and the influence of the first two is especially to be dreaded in this direction.

Treatment.-When there is any marked tendency to tubercular disease in the mother, she ought never to be allowed to nurse her child, but it should at once be provided with a nurse, and any appearance of a scrofulous habit in the child should be attended to early. In such cases, also, such diseases as measles, hooping-cough, and all pulmonary diseases, should be most carefully treated. If, in spite of prophylactic treatment, tubercular disease should become established, the greatest attention must be paid to the hygienic condition of the child. Its food must consist exclusively of what is nourishing and of easy digestion, such as meat, eggs, milk, \&c.; beer and small quantities of wine; its clothing must be warm, but not impermeable to air ; it should be removed to fresh air, yet carefully guarded from cold, and, when possible, it should be removed during winter to a warmer climate. Cod-liver oil, when it can be borne, and the whey treatment, are our most efficient methods of cure, but unfortunately they very seldom effect it, and in most cases all we can hope for is an arrest of the disease, an increase in weight, and an improved condition of the patient for a time. The tubercular condition of various organs may require special symptomatic treatment, which will be found in its proper chapter. The febrile syriptoms may often be mitigated by the administration of quinine and digitalis, either alone or in combination, and the irritating cough may be much relieved by opiates-hyoscyamus or belladonna; and for the diarrhœa, astringents, such as acetate of lead, tannin, \&c., must be used in combination with opium. The hectic sweating may 
be greatly diminished by inunctions and the internal administration of quinine and tannin, or small doses of atropia.

\section{Purpura}

Rilliet and Barthez have divided the cases of this disease into two groups, which are not, however, very sharply divided. These are-

a. Purpura simplex, including cases of skin ecchymosis not complicated by hrmorrhage from mucous surfaces ; and

b. Purpura hæmorrhagica, morbus maculosus Werlhofii, in which the petechiæ are accompanied by hæmorrhage from the mucous tracts.

Purpura simplex is characterised by small spots about the size of a linseed, of a dusky red colour, and which do not disappear on pressure; these extravasations may also be punctate (petechiæ), or streaky (vibices), or the hæmorrhage may be diffuse, and extending over large surfaces (ecchymosis). Any of those eruptions may be limited to some particular part, as the extremities, joints, the abdomen, or they may cover a large part of the surface; they may also appear once only, or in consecutive crops.

In purpura hæmorrhagica the ecchymotic cruption occurs with greater irregularity, usually in consecutive outcomes, and sometimes an actual hæmorrhagic oozing from the skin occurs, as I have seen twice in the cases of two girls, aged four and five years, in whom an abundant loss took place from the skin of the forehead and temples.

Mucous hæmorrhages always occur in this disease, and they have the following order of frequency:

Epistaxis, or bleeding at the nose, is sometinies the first symptom, and may be of serious extent, lasting sometimes for hours.

Stomatorrhagia, or hæmorrhage from the mouth and throat, generally arises from the gums, but it may also come from the tonsits (Ferris).

Enterorrhagia, or hæmorrhage from the intestines, is pretty frequent, consisting either of only slight admixture of blood with the evacuations, or of the passage of large masses of dark 
chocolate-coloured clot, preceded by smart colicky pain. Hiemorrhagic erosions, deep ulceration, or even gangrenous destruction of the mucous membrane, may be found on post-mortem examination.

Hæmatemesis, or vomiting of blood, is less common than the above-mentioned variety of bleeding, and here the amount of blood lost is not usually great, being generally limited to a slight intermixture with the vomited matters, though sometimes the loss is very considerable. The mucous membrane of the stomach will be found, after death, to have patches of ecchymosis scattered over it.

Hæmaturia, or passage of blood in the urine, is often very serious, and I have noticed it in about a third of all the cases I have seen.

Hæmoptysis, or hæmorrhage from the lungs, is not very common, nor is the hæmoptoic infarctus.

Ecchymosis under the conjunctiva is seen now and then, and in one case I saw hæmorrhage from the eyes and ears; and in very severe cases extravasation may be found, on post-mortem examination, in the brain and serous membranes.

Symptoms and course.-Purpura usually runs its course almost free from fever, though in some cases there is wellmarked pyrexia, and the child complains for a few days previous to the hæmorrhagic eruption of a general sense of weariness and lassitude. Sleep is generally restless and disturbed, the appetite impaired, and when the loss of blood is abundant the skin becomes pale and waxy, the pulse small and irregular, the child is giddy and faint, hæmic murmurs accompany the sounds of the heart, convulsions may occur, and passive dropsy is frequently established. The disease may run its course in a few days, though more usually it lasts some weeks.

Schönlein has indicated a special form of purpura under the term Peliosis rheumatica, occurring chiefly amongst the poor and in cold weather. It is usually ushered in by a feeling of lassitude and pain in the joints lasting from two to four days, followed by the appearance of a petechial eruption, which is more abundantly distributed in the neighbourhood of the affected joints. The joints themselves are swollen and very painful, especially on being moved, and they present all the appearance of joints affected by acute articular rheumatism. 
In one case which I examined after death, I found in the knee- and ankle-joint effusions of blood of both old and recent date; but, according to other observers (Traube), the joints have been found altered only as they are in acute articular rheumatism.

Peliosis rheumatica is generally accompanied by symptoms of fever, and Kaltenbach's temperature observations would tend to show that the fever, at the height of the disease, is intermittent; the exacerbation of the fever occurring at first during the early hours of the morning just after midnight; whilst later it takes place in the evening, and the process of defervescence is marked by an extension of the intervals. Besides the hrmorrhagic spots in the neighbourhood of the joints, I have noticed in several cases mucous hæmorrhages, hæmaturia, and the passage of blood from both stomach and bowels.

Causes.-But little is known of these, more than that purpura is sometimes a primary disease, and sometimes secondary to some other. A congenital hæmorrhagic diathesis (hæmophilia), due, apparently, to an abnormal fragility of the capillaries, has been noticed as occurring in certain families, and acute fatty degeneration of the liver may give rise to similar symptoms, as has already been men tioned. When the disease occurs later in life we must suppose it to be due to some change in the capillaries, in the blood, or in the circulation, occurring either primarily or during the course of some other disease, such as tuberculosis, scrofulosis, chronic intestinal catarrh, typhoid, measles, icterus, \&c.

Bohn states, and I believe with some truth, that the hæmorrhagic spots are the result of capillary embolisms, and there certainly is a class of cases to which this explanation is applicable, one of which has just recently come under my notice. It occurred in a boy suffering from scrofulous caries, who became affected with extensive ecchymosis and deep blue colouring of the skin of the lower part of the right thigh, following severe pain. A few days after that attack, when the colour of the skin had become somewhat restored, a crop of dark spots, of the size of linseeds, appeared, which afterwards became crater-like ulcers and were extremely difficult to heal.

Insufficient and improper food, together with residence in cold, damp, and badly ventilated dwellings, have also a considerable 
influence in the production of purpura, as well as of other diseases.

As a secondary affection purpura simplex will be met with in children of all ages, but purpura hæmorrhagia and peliosis rheumatica occur only in children between four and twelve years of age. Girls seem to be attacked somewhat more frequently than boys, and the disease is most common in spring and autumn, especially when the weather is bad.

Those who suffer from the hæmorrhagic diathesis generally have an attack of bleeding once or twice a year; and in girls I have seen the tendency disappear entirely when menstruation had become established.

Prognosis.-Purpura simplex, when primary, has usually a favorable issue, but when secondary its indications are always unfavorable. Purpura hæmorrhagica is a disease much to be dreaded under all circumstances, though sometimes recovery takes place after the gravest symptoms have appeared, even after gangrene of the intestinal mucous membrane, if the external relations of the patient be favorable.

Treatment.-The patient must be kept at absolute rest in a diry, well-ventilated room, the diet must be nutritious and of easy digestion, and must include an abundance of fresh vegetables, the juice of acid fruit, such as limes and lemons; wine and beer. Quinine and the mineral acids should be given internally; Henoch has recommended ergotin, and in case of hæmorrhage from mucous surfaces the perchloride of iron and ice may be given internally or locally applied, as the case may require. In severe epistaxis the nostrils may be plugged. Baths containing vinegar are useful.

\section{Rheumatism}

Of the large group of rhcumatic diseases almost the only one met with amongst children is acute articular rheumatism, and with them this disease differs very little from the characters it presents amongst adults, save that it seldom occurs with the same severity and obstinacy.

On post-mortem examination the joints may be found affected by simple hyperæmia, swelling of the synovial membrane and 
adjacent counective tissue, and an increase in the amount of the synovial fluid, or the cavity may be found occupied by profuse suppuration. The epiphyses of the bones constituting the affected joints are also found to be hyperæmic, and Griesinger has found purulent infiltration of the bones. In the blood there will be found a diminished amount of fibrin and an increase of the albumen, and the heart and pericardium are often affected by inflammatory action, this being especially apt to take place in the valves.

Symptoms and course.-An attack of articular rheumatism is usually, though not invariably, ushered in by shivering, febrile symptoms, restlessness, and sleeplessness, though occasionally the local affection of the joints occurs as the first indication of the disease. The joints most frequently involved include the knee, ankle, wrist, and elbow, and several of them are generally attacked simultaneously; and a very characteristic symptom is the pain which is excited by any pressure or attempt at motion in the limbs. The skin of the affected limbs may either be slightly redder than usual, or of its normal colour.

The formation of pus in the joints is not often seen in children, the inflammatory action generally stopping short of that, and disappearing after a course of varying duration, though the metastatic tendency of the inflammation is very marked in children.

'The fever is generally remitting in degree, and varies much in severity in different cases. There is a considerable tendency to perspiration, though this is not so marked as in adults, neither are sudaminal eruptions so common. The urine is diminished in quantity, darkly pigmented, has abundance of sediment, and contains an excess of uric acid and urates.

The most frequent and important complications in children are endo- and pericarditis, occurring, according to my own experience, in three fifths of all the cases, almost all the cardiac lesions of childhood being due to this cause. The pleura and the meninges of the spinal cord are also sometimes affected, the latter being followed by the establishment of chorea minor. Peri-endocarditis may set in with the earliest indications of the disease, or it may appear at a later stage. Its occurrence is usually indicated by an exacerbation of the fever without any fresh joint-affection, restlessness, anxious expression of face, 
vomiting, and even delirium, and pain over the heart. Physical examination will at once discover it, and this precaution ought to be taken every day in a case of acute rheumatism. The relation of this disease with chorea has been referred to in a previous chapter.

Cases of articular rheumatism of a slight character, and free from complications, get well in from ten to fourteen days, whilst those in which cardiac inflammation sets in seldom are convalescent under five or six weeks. There are some obstinate cases which alternate between better and worse, and death alone seems able to relieve them from their incurable disease.

Rheumatism is not a disease of early childhood, and is most usually met with after five years of age, though occasional instances of it are met with in younger children.

Causes.-These are yet but little known, though it is quite certain that barometric and thermometric variations and exposure to cold have a direct influence in its production. It seems to have a strongly hereditary tendency, as is well illustrated by a case in my own experience, where, of twelve children of a mother who had suffered from acute rheumatism and heart complication, eleven had the disease before they were twenty years of age.

There is scarcely any possibility of mistaking this disease for any other.

Treatment.-There is no specific by which this disease can be combated, so that the treatment must be symptomatic and directed to the amelioration of the fever and the pain in the joints, and to obviate cardiac complication. For the mitigation of the fever infusion of digitalis and tincture of veratrum viride and quinine seem to have given the most satisfactory results, the latter being contra-indicated when there are any cerebral symptoms, giddiness, or irregularity or retardation of the pulse.

For the relief of the pain and restlessness opiates must be given, in form and quantity suitable to the age of the child. From a combination of quinine and belladonna $I$ have often seen great relief. For the local pain cold water packing has been recommended, or the wrapping the joints in wadding or tow, and their inunction with a mixture of oil and chloroform, or an ointment of digitalis and belladonna.

[Blistering immediately above the 
affected joints is a plan now in much favour in England.L. T.]

When cardiac inflammation is threatening counter-irritation over the heart is advisable, and when the mischief is permanent great care is necessary until compensatory action of the heart has been established. 


\title{
EIGHTH DIVISION
}

\author{
ZYMOTIC DISEASES
}

\section{Scarlet Fever}

THIs is an acute contagious disease, affecting the system generally, but with an especial action on the throat and kidneys of a catarrho-inflammatory character.

The post-mortem appearances in fatal cases of scarlet fever belong in part to the disease itself, and partly to its complications and sequelæ, and most of the latter will be found treated of in other chapters. The blood is found to be fluid and of a dark bilberry' colour, with a diminished quantity of fibrin; the kidneys always in a state of catarrhal or acute inflammatory alteration; the spleen, lymphatic glands, and intestinal follicles swollen; or there may be lymphatic new growth (lymphoma) in the liver, spleen, kidneys, and especially in the intestinal canal (Wagner). Fatty degeneration of the liver, heart, muscles, and brain, are also constantly found as the result of the high temperature of the disease.

Symptoms and course.-The period of incubation is from two to ten days, during which the patient may be either perfectly well or suffering only from a feeling of lassitude.

Stadium prodromorum.-This is marked by feverish symptoms, shivering alternating with heats, a pulse of perhaps 160 , and temperature sometimes as high as $41.5^{\circ}$, general discomfort with headache, sickness and vomiting of bilious-looking stuff, occasionally epistaxis, pain in swallowing, and redness of the tonsils and fauces. A diffuse punctate reddening is also generally developed in the soft palate after the access of the angina. In some epidemics the disease is ushered in by violent vomiting and purging and symptoms of collapse; and in severe cases delirium and coma are frequent symptoms. 
The prodromal stage may last only a few hours up to two days, or may even never be manifested; and from the severity of the condition in this stage, some idea may be formed of the probable course of the disease.

Stadium eruptionis.-The eruption appears first at the root of the neck and upper part of the body, and with its development the temperature rapidly rises. The rash is red and punctate, disappears on pressure, and reappears from the periphery to the centre of the finger-mark. It spreads with increasing intensity over the lower part of the breast, back, abdomen, and extremities, and the skin, during its existence, is markedly swollen. It generally appears on the joints a short time before it is seen on the other parts of the limbs, and it is not often seen on the face. Simultaneously with the appearance of the eruption the condition of the throat becomes worse, and the tongue becomes rough, dry, and intensely red, a condition which is known as the "strawberry tongue," and is due to the swelling of the papilla.

The high temperature is retained during this stage with slight remissions, these occurring more frequently in the evening than in the morning; and albumen and shed epithelium may also then be often found in the urine. In many cases, especially in particular epidemics, considerable distress is felt from itching of the skin.

As the temperature and the pulse fall, and the distress in the throat becomes less, the eruption fades after a continuance of two or three days into a yellow or yellowish-brown colour, and finally disappears.

Stadium desquamationis.- This is marked by a loosening of the epithelium which begins at the parts on which the eruption first appeared, and the scarf-skin may come off in large lamellæ (desquamatio membranacea), or in small scaly particles (d. furfuracea), or in severe cases the hands and feet may shed the epithelium as complete investments, appearing like gloves, and including the nails. I have twice seen the deeper epithelial layer shed and the vascular cutis laid bare, as if the patient had been burnt or scalded.

During this stage sweating generally occurs, the urine becomes more abundant with a profuse shedding of epithelium, and sleep and the appetite return. The duration of the desquamative 
stage may vary from eight to twenty days, though the shedding may be in some cases so slight as scarcely to be noticed.

What has been said above refers only to the course of the simple and mild disease, but it may be very much altered by an anomalous course, complications, and sequelæ.

The eruption is not always spread over the skin regularly (S. lævigata), but may be confined to certain parts (S. variegata), or the exanthem may take the form of small nodules (S. papulosa). Sometimes the epidermis is raised in small bladders (S. miliaris), or vesicles of larger size (S. bullosa, $\mathbf{S}$. pemphigoidea), as I have noticed several times on the face. In some very exceptional cases the eruption appears only at certain times, with an evident intermittent character (S. intermittens), or the eruption may never be visible (S. sine exanthemate). Occasionally we see small extravasations of blood in the skin accompanying the rash (S. hæmorrhagia, S. petechialis), and now and then we see urticaria associated with it. I believe I have observed one phenomenon of a curious but yet doubtful character, presenting sharply marked insular portions of the skin of a milk-white colour, or at least much whiter than the rest of the integument, and which $I$ regarded as due to a temporary paralysis of the arterioles.

The angina is not always simply catarrhal but may become parenchymatous, or everi result in suppuration and be accompanied with distressing difficulty of swallowing and breathing, with snoring respiration and a dulled nasal voice. In some epidemics and under individual idiosyncrasy, the sore throat may take on a croupo-diphtheritic character with a tendency to necrosis or even gangrene. The inflammation and its consequent exudation may extend over the uvula, palate: and fauces, and thence into the nostrils, larynx, œsophagus and stomach, and also through the Eustachian tube into the internal ear, where it may lead to destruction of the mechanism of hearing and to permanent deafness.

Angina scarlatinosa is generally accompanied by swelling of the submaxillary glands and of the cellular tissue of, the neck, which often runs on to the formation of abscesses, or even to gangrene. Cases have occurred where, in opening these abscesses, the large blood-vessels have been injured, and the hæmorrhage has been fatal. 
Diphtheritic septicæmia, the result of the resorption of the necrotic detritus in the throat, is almost always fatal.

Conjunctival diphtheritis, with pan-ophthalmitis and rapid destruction of the globe, has occurred several times in my practice during the course of scarlet fever, and I have also seen diphtheritic and gangrenous vulvo-vaginitis.

The kidneys always suffer more or less in cases of scarlet fever, the usual condition being that of simple catarrh, but it may be excited into parenchymatous nephritis by external influences, such as exposure to cold, error in diet, \&c.; and the abundant epithelial shedding may produce mechanical obstruction of the renal tubules. The usual period for the onset of scarlatinal nephritis is between the thirteenth and twenty-first day of the disease, and it is indicated by the occurrence of dropsy, albuminuria, and the other uræmic symptoms which are related in their proper places.

The inflammatory condition of the kidney bears no direct proportion to the severity of the attack of scarlet fever or to the quantity of eruption, as it is often most severe in cases which have passed almost unnoticed. The percentage of kidney complications in scarlet fever varies from five to seventeen. Frerichs has described a rare form of dropsy without any disease of the kidneys, occurring after scarlet fever, which he believes to be due to paralysis of the cutaneous nerves by exposure to cold during desquamation; and I have lately seen one such case, where repeated examination of the urine revealed no change, whilst there was very acute dropsy of the skin, without any effusion into cavities, which lasted twelve days.

A very dreadful kind of scarlet fever is every now and then met with, where the child succumbs almost directly to the most violent symptoms, without the forewarning of any prodromata. These cases sometimes run their course from beginning to end in thirty-six or forty-eight hours, almost even before the eruption has appeared, the only symptoms being vomiting, loss of consciousness, coma, violent delirium, or convulsions, with a most abnormal and continuously high state of the temperature. They are generally the forcrunners of an epidemic, and are not unfrequently seen in children who are very healthy.

On post-mortem examination the brain is found to be hy- 
peræmic, as are also the meninges and kidneys, with scarcely any other perceptible change.

Amongst other complications of scarlet fever may be mentioned croupous bronchitis, pneumonia, pleurisy, pericarditis, endocarditio, meningitis, with the formation of pus in some of the serous cavities, and pulmonary gangrene.

I have seen hemiplegia occur in the case of two boys, between eight and nine years of age, the paralysis occurring in both cases after severe uræmic convulsions, and in one it was accompanied by well-marked aphasia. In both it was probably due to some cerebral hæmorrhage, and in both recovery took place.

Arthritis scarlatinosa is occasionally met with in certain epidemics of scarlet fever, occurring sometimes during the eruptive stage, but more usually during desquamation. It seems to affect the knee- and elbow-joints by preference, and more rarely the hip, ankle, and shoulder ; is excessively painful, and generally results in suppuration and pyæmia, or if the children recover, in caries and contractions of the joints. ${ }^{1}$ Periostitis, ostitis, and necrosis, also occur in some of the bones, as I have lately seen in the instance of the upper jaw.

Scarlet fever may be complicated with other exanthemata, especially varicella, more rarely with variola and measles, so that the symptoms of the two diseases may run side by side, as has been recorded by Monk, Thomas, myself, and others. Under such circumstances the cases are usually severe.

As sequelæ of scarlet fever may be mentioned hypertrophy of the tonsils with a tendency to recurrence of acute tonsillitis, chronic nasal catarrh resulting in ozæna, diseases of the ear with chronic otorrhea of one or both ears and temporary or permanent deafness, and when the deafness is complete in very young children they remain dumb. But the sequela most to be dreaded is disease of the kidneys, which may take a chronic form, subject at any time to an acute exacerbation which will probably prove fatal.

Noma of the face and genitals is more rare after scarlet fever

1 I have lately seen an instance of this affection in consultation, in the case of a young lady, and I have no doubt that the complication is really due to a pyæmic process of a less acute kind than is seen to carry the patients off with such rapidity when abscesses "orm round the throat.-L. T. 
than after measles. After severe cases of scarlatina there often remain a very persistent weakness and anæmia, which may be so serious as to prevent children, even eight or twelve years old, from getting about for weeks.

Causes.-The disease is eminently contagious, and usually occurs epidemically. Its contagium is as yet unknown, and though Bullier describes a micrococcus as existing in the blood of patients suffering from scarlet fever, yet it cannot be regarded as the cause of the disease or its infection-carrier, but is much more likely to be the result of fermentative or catalytic action in the tissues. It is perfectly certain that scarlet fever may be communicated by contact with intermediate persons, and with fomites, and the latter may retain the poison for a long time. There seems also to exist in some people a peculiar disposition to be affected by the poison.

The majority of cases occur in children over two years of age, sucklings being seldom affected, though I have seen children as young as eight weeks attacked. Second attacks of scarlet fever are rare.

Diagnosis.-The symptoms which have been detailed above will generally be decisive enough to leave no doubt in a case of scarlet fever, especially if it be known that the disease is rife in the neighbourhood. But in some cases it is not well marked, and it is possible to compare it with primary diphtheritis, the eruption of iodism, and with the congestive erythema of pneumonia. It is scarcely possible to mistake it for measles if the disease be uncomplicated and attention be paid to the differing characters of the eruptions, though it may happen when the eruption is very slight and transient, or if it be complicated in any way.

The prognosis must always be cautious, for scarlet fever in its mildest form is always a serious disease, and only after the third week, when desquamation has been completed, can any patient be considered safe. Any complication, such as diphtheritic inflammation, nephritis, or the appearance of intense bloodpoisoning, must be regarded as of a most unfavorable nature.

Treatment.-The most rigid isolation must always be provided for a case of scarlet fever, as a precaution against its spreading. For slight cases a dietetic regimen is almost all 
that is needed, as in other respects they may be left to nature. The room should be kept at a regular temperature of $15^{\circ}$ to $18^{\circ}$ Cent., with free ventilation; the bed should be.warm, but not overclad, and the body and bed-linen should be changed daily, though this latter point may be objected to both by parents and practitioners. Copious drinks of water, lemonade, juice of fruit, milk; and beef tea, should be allowed; and as long as the desquamation lasts the patient should be kept in bed.

The difficulties and complications of more severe cases must be treated symptomatically, it being always borne in mind that there is no specific for scarlet fever. For the reduction of the high temperature, cold baths or cold wet packing, when they can be used, are undoubtedly the best means; or Schneeman's plan of rubbing the patient over with lard may be tried, but though it does reduce the temperature it seems to have but little else to recommend it. Of drugs the most useful are the mineral acids, digitalis, and quinine.

When the urine becomes albuminous all remedies which act directly on the kidneys should be avoided, especially when the urine becomes dark in colour and diminished in quantity, and only simple acids should be given. When the blood has disappeared from the urine, such diuretics as digitalis, juniper, and acetate of potash, may be given, together with acid drinks or effervescing waters.

Anasarca may be treated by lukewarm baths, given twice a day, diaphoretics, and diuretics, and, when it becomes extreme, by hydragogue cathartics. If uræmic symptoms appear quinine in large doses, one to two grains every six hours, and cold packing will be found of service; and in case of symptoms of collapse setting in, stimulants must be freely administered. When parenchymatous inflammation attacks the glands and cellular tissue the best application will be found in warm poultices, and if any induration remain after recovery, some deobstruent, as tincture of iodine, should be used locally.

As has already been advised, the urine should be carefully examined from day to day until the stage of desquamation has completely passed, for till that time no case of scarlet fever is free from danger. 


\section{Measles, Morbilli}

This disease may be termed a general zymotic affection in which there is catarrh of the respiratory mucous membrane and a peculiar red eruption slightly raised above the level of the skin.

In fatal cases the skin is found, after death, to have a livid marbling, to be finely roughened, and sometimes to be scattered with hæmorrhages. The respiratory organs are marked by the appearance of catarrhal inflammation and often by croupodiphtheritic exudation, and a punctate reddening has been found on the mucous membrane of the mouth, larynx and bronchi, and sometimes also on the mucous membrane of the small intestines in children who have died during the stage of eruption, and which has been described by some authors under the term enanthem. The blood is always fluid, dark, and deficient in fibrin.

In measles we also find, as in scarlet fever, but not to the same extent, acute fatty degeneration and lymphoma in certain glandular organs.

The incubation of the disease varies from eight to ten days, and during that time the patient may feel quite well, or suffer only from shivering and malaise.

Stadium prodromorum. - At first the symptoms consist only of slight recurrent shiverings with rise of temperature, reaching its maximum within twelve or twenty-four hours, and having remissions usually towards morning.

'There is a general sense of lassitude, pains in the head, limbs, and joints, alteration in the appearance of the face, loss of appetite, disturbed sleep, together with a catarrhal affection of the mucous membranes of the nose, eyes, and air-passages, indicated by sneezing, lachrymation, cough, hoarseness, and pain in swallowing, and in some cases we see delirium and coma. This stage may last from three to fourteen days, and a day or two before the outbreak of the eruption a spotty or streaky reddening may be seen on the soft palate.

Stadium eruptionis.-The appearance of the rash is generally marked by an exaltation of temperature, to $40^{\circ}$ or even higher, 
and symptoms of cerebral congestion, occasionally even delirium and convulsions.

It appears first, in most cases, on the cheeks and temples, the face being somewhat swollen, and at first is seen as a transient punctate redness which increases in extent and colour, the points becoming livid and confluent, the resulting spots always leaving between them islands of unaffected skin. The eruption extends over the neck, trunk, and extremities, for twentyfour or forty-eight hours, the temperature rising meanwhile and the catarrhal symptoms increasing in severity. After the rash has got to its height it remains for twenty-four or thirtysix hours, and then begins to lose its colour, becoming yellowishred and then a dirty yellow, the defervescence accompanying its disappearance gradually, or with some interruption, until it has gone entirely on the third or fifth day. Coincidently, the photophobia and the catarrhal secretions from the nose and ejes disappear, and a moist cough sets in, accompanied, in older children, with a lumpy expectoration ; sleep and appetite return and an abundant diuresis sets in.

Stadium desquamationis.- Eight or ten days after the appearance of the eruption all trace of it has usually disappeared and the epidermis becomes loosened in scales, the fever has been resolved either by crisis or lysis, and the catarrhal symptoms finally exhaust themselves.

Anomalies and complications.-The eruption may vary from its general character in that the spots become more than usually confluent, and resemble the rash of scarlet fever more than that of measles (morbilii conferti v. confluentes); or they may become hæmorrhagic (morbilli hæmorrhagici); or by partial rupture of the distended capillaries the spots may take on a bluish colour, which does not disappear on pressure, and which may remain as a pigmentation for some weeks after the eruption has disappeared.

Sudden disappearance of the rash during the florescent stage, the so-called in-striking of the eruption, is often seen when there is some inflammatory complication of internal organs, such as extensive bronchitis, broncho- or croupous pneumonia; also after profuse epistaxis ; and, of course, it may be that the disappearance of the rash really stands in the relation of cause to these diseases. 
Cases of measles without eruption (morbilli sine exanthemata) are seen during every epidemic; and also other cases, though much more rarely, without any catarrhal symptoms.

The most frequent and important complications in the florescent and desquamative stage are those affecting the respiratory apparatus, such as diffuse capillary bronchitis, atelectasis of the lower and posterior margins of the lungs, brouchopneumonia, gangrene of the lungs, œdema of the glottis, croupous laryngitis, and pleurisy. Less common complications are seen in blenorrhagic and diphtheritic conjunctivitis, keratitis, diphtheritis of the nose and throat cavities, simple or croupous inflammation of the tonsils, croupo-diphtheritic osophagitis and gastritis, intestinal catarrh, dysentery, and occasionally albuminous nephritis, and noma.

I have several times seen measles and varicella, measles and scarlet fever, and even measles and smallpox existing coincidentally in the same patient; and under such circumstances the one disease seems to have exerted a somewhat mitigating influence on the other.

The sequelæ have already been partly detailed in speaking of the complications, but there may also be mentioned here hoopingcough, especially if both diseases be epidemic at the same time, hyperplasia and cheesy degeneration of the lymphatic glands, chronic pneumonia, pulmonary phthisis, bronchiectasis, tuberculosis, especially in children belonging to a family with a tubercular or scrofulous taint, chronic catarrh of the nasal mucous membrane, diseases of the ear, periostitis, and diseases of bone. Measles seems to have an aggravating effect on all scrofulous diseases.

Causes.-This disease undoubtedly originates and spreads by means of some poison, but what its nature may be is yet unknown. Hallier has described a microscopic fungus in comnection with measles, the mucor mucedo, but this is much more likely to be the result of the disease than its cause. Inoculation with the blood of a patient suffering from measles has produced measles in a healthy person (Katona and Speranza), and the mucus from the nose and air-passages, as well as the tears, seem to have the power of communicating the disease. The communicability of the disease seems quite as ready luring the prodromal as in the later stages, and at certain times and in certain 
places the contagion seems peculiarly powerful. There seems to be a certain amount of periodicity in the epidemics of measles, the less severe seeming to occur every three or five years, whilst those of a more dangerous type make their appearance every seven or eight years, whilst in large towns there seem to be always a few cases going on which burst out into an epidemic on certain alterations of the weather. It seems to attack sucklings very rarely, especially during the first six months, though I have met with it in children only four or five weeks old.

One attack does not seem to confer absolute immunity from it for the future, as I have several times seen measles twice in the same patient within a few weeks, nor do other acute or chronic diseases afford any protection from it. In children's hospitals it is not at all unusual to see a patient suffer from two or three different exanthemata immediately one after the other, the eruption of a second appearing almost before that of the first had vanished.

The varying characters of different epidemics, some being of the sthenic and others of the asthenic type, seem to depend on the conditions of the weather, the time of year, and other conditions which are not so well known. As a rule, epidemics occurring during summer are less severe than those in winter.

Diagnosis.-The disease with which measles may most readily be confounded is Rötheln (Rubeola); but the latter should readily be recognised by its slight degree of fever, by its rapid disappearance, and also by the absence of the catarrhal symptoms and of the peculiar raised character of the eruption of measles. In roseola syphilitica the course of the disease is entirely free from fever, the eruption remains longer and becomes infiltrated, and there is almost always some other indication of the specific disease. Erythema urticatum may be distinguished from measles by the frequent recurrence of the eruption in peculiar wheals accompanied by troublesome itching of the skin, and the complete absence of the catarrhal symptoms. Between variola or scarlet and measles the differences are always so well marked that it is scarcely possible to make any mistake.

Measles is generally a disease which ends favorably, though in the prognosis the character of the epidemic and the pecu- 
liarities of the patient must always be taken into account; for whilst I have known 200 cases to occur in a summer epidemic in the country without a death, I have also seen ten children die out of twelve in the Prague Hospital when the disease had a diphtheritic tendency.

Treatment.-Although it is almost certain that everybody must have measles at least once, it is always advisable to isolate children who are affected by it, and to avoid the extension of the disease in every possible way, especially during epidemics of an unfavorable type. Whenever it breaks out in a collection of children the institution should be emptied as soon as practicable.

The treatment of the early stage differs little from that required by other fevers, save that the catarrhal affections of the eyes may demand that the sick chamber be darkened. So lung as fever is present the diet must be limited to milk and broth, and care must be taken that the bowels act regularly. Some slight calmative may be given to relieve the irritation of the cough, such as a small dose of Dover's powder or syrup of poppies. When the cough is very severe a little ipecacuanha or antimony may be added, or if the bronchial secretion be very profuse they may be given in emetic doses. When the fever is very intense quinine and digitalis should be given, and in the event of any appearance of collapse stimulants may be used freely. Any typhoid symptoms should be treated by liberal stimulation, and the administration of quinine and the mineral acids. As long as the rash remains the child should be kept in bed, or at least in the sick-room, especially during cold weather.

\section{Rötheln, rubeola}

This disease cannot be identified either with measles or scarlet fever, for its course is either not marked at all, or only very slightly, by general symptoms, or by anything beyond a transient spotty redness of the skin. It is not of frequent occurrence, and almost always appears in an epidemic form. Its period of incubation is from ten to fourteen days, and its initial stage is very short, being from a few hours to two days at the longest, and is marked by shivering and heats, pain in 
the head, and, according to some authors, by catarrh of the nose, throat, and eyes. The eruption usually appears suddenly and over the greater portion of the skin, and consists of bluish-red roundish spots of varying size, which become here and there irregularly confluent, are scarcely at all raised above the level of the surrounding skin, and which disappear under pressure but reappear rapidly.

The eruption may last from twenty-four to forty-eight hours, and is followed neither by roughness nor pigmentation. Several observers notice that a few days after the disappearance of the eruption a furfuraceous desquamation has taken place. The whole course of the disease is remarkably free from fever, the increase of temperature being seldom more than one degree, and that falling as soon as the eruption has appeared. Emminghaus has recorded cases which have been complicated by pneumonia and have had a fatal issue, but they seem to me to have been cases of measles rather than of rubeola. Sex does not seem to make any difference in this disease, and $I$ have seen children from eight months to ten years old affected.

I am not yet certain that the disease is coritagious, though other observers assert that it is communicable directly and indirectly from one individual to another. It certainly, however, does not confer immunity from measles, its prognosis is always favorable, and it does not want much treatment.

There is a form of circumscribed erythema which is sometimes seen in children during the first two or three weeks of life, probably the result of the general change going on in the skin, which is not identical with rötheln, but may readily be mistaken for it. The skin is scattered with numerous nodules and spots of an intense red colour, which appear equally on the face, body, and extremities. There are never any catarrhal or feverish symptoms, and the rash entirely disappears in a few days.

\section{Variola, smallpox}

This is an acute contagious disease which runs a typical course, and is marked by an efflorescence on the skin which begins as nodules and papules and ends in pustules and crustation. 
Concerning this eruption two opinions are held, one originating from Hebra, that there is only one eruptive process, and that it appears in three forms-according to its intensity-the variola vera, variolois or variola modificata, and varicella. The other, which seems recently to have gained considerable support, is that variola and variolois are quite distinct from varicella. Thomas says that varicella is a disease sui yeneris, and bases his opinion on the following facts: that the appearance and accession of the eruption are much more rapid, that the epidemics of varicella are much more frequent than those of smallpox, that vaccination does not confer immunity from varicella, and that in the latter disease there are no prodromal symptoms, the eruption often appearing at the end of the first day. Further, the contents of the papules of varicella are not inoculable, and the period of incubation is not constant as in smallpox. From my own observation, based on very considerable experience, gained especially during the epidemic of 1872 , I am inclined to separate variola and varicella, and to regard the latter as a wholly independent disease. My chief reason for this view is that the two diseases do not seem to confer immunity from one another. I have seen them follow one another with so short an interval as two or three weeks, that vaccination does not protect the patient from varicella, and that inoculation with the matter from the pustules of varicella produces only the same disease, usually after a period of eight days' duration.

Anatomy-According to most observers, especially Auspitz and Bach, the first stage of the eruption consists of the formation of nodules about the fifth day, due to the raising up of epidermis from a swelling of the Malpighian layer, the cells of which, and their nuclei, are enlarged. The vessels of the corium, both in the papillary layer and beneath, are dilated, and their walls scattered with numerous small round cells, which are also found in the stroma of the papillæ; the latter, however, remaining themselves, along with the glands, unaltered.

Further in the course of the eruption these nodules become papules, a distinct meshwork of fibres appearing under the evidently swollen cells of the Malpighian layers, which include some pus-cells. The papillæ become broader and shorter, and 
the vessels are surrounded by cells. By the extension of this meshwork, and the increase of the contained pus-cells, the pustular stage of the eruption is arrived at, the contents of the pustules being, as it were, encapsulated between two layers of epithelial cells ; and the outer layer being gradually destroyed, the contents being extruded and becoming dry, the crust is formed beneath which the ulcerated surface heals. A mark is left, which is not of itself, however, characteristic of smallpox, for other pustules leave similar abrasions.

Symptoms and Course.-The disease follows a mild or severe course in proportion to the amount of the eruption and the accompanying complications. Its period of incubation varies from ten to fourteen days, and during that time the patient may give no evidence of suffering, or may be disturbed by a feeling of cold, slight headache, and a general sense of lassitude.

Stadium prodromorum.-The symptoms seen in this stage are those which are common to other febrile diseases, such as shivering, exalted temperature, gastric disturbance, furred tongue, squeamishness and vomiting, pain in the head, great restlessness, alternating with drowsiness, grinding of the teeth, neuralgia especially of the intercostal nerves, and sometimes grave nervous symptoms, such as delirium and convulsions. Occasionally profuse sweating occurs as a symptom, or a transient erythematous reddening of the skin may be seen. In the case of a boy aged eight years I saw well-marked mania as one of the prodromata. The characteristic pain in the back and in the pit of the stomach, which is so constantly met with in adults, is by no means so common in children, being absent quite as often as it is present; whilst children, especially those who are somewhat advanced, frequently complain of pains in the limbs, especially in the joints. Early indications of the eruption are sometimes to be seen in the mouth during this stage.

Stadium eruptionis. - The eruption makes its appearance amidst a gradual increase of the severity of the fever symptoms and a further exaltation of the temperature. It is usually seen first on the face, and in varioloid it consists of small, hardish, red nodules, sparsely scattered and isolated, though here and there they may be gathered in groups, ending after two or 
three days' duration in vesicles, and, by purulent alteration of the contents, in pustules.

In variola vera these changes occupy eight to ten days, but in the modified smallpox they may be completed in four or six. Coincidently with the appearance of the eruption a well marked remission of the temperature and pulse will be observed, provided there be no complication of an inflammatory character; but when the pustular alteration of the contents of the vesicles begins the temperature rises again, as ligh, perhaps, as $40^{\circ}$ or $41^{\circ}$, the face swells, and an intolerable burning and itching of the skin gives rise to great suffering to the patient.

Stadium decrustationis. - In variola vera the pustules continue till the fourteenth of even to the eighteenth day, and in varioloid to the seventh and twelfth. After such duration their contents dessicate and form a brownish thick crust, which gradually separates and, on falling off, leaves a cicatrix covered by recent epidermis. 'The marks left by smallpox sometimes disappear in children, but they usually remain for life.

According to the form, contents and distribution of the pustules, several varieties of smallpox have been defined, which, though not of great practical value, are worth remembering. Of these may be mentioned the following: smallpox-measles (Blattermasern), Nirlus, a form of a very mild kind in which pustules are never formed; variola milliformis, in which the eruption is papillary and without suppuration or inflammatory action-a most fatal form, and noticed by myself only in children who were atrophied, anæmic, or otherwise diseased; $V$. pemphigoidea, in which the eruption takes the form of large bullæ; $V$. confluens, in which the pustules run together and produce large suppurating excavations; Rupia variolosa, in which, during the stage of decrustation, the halfdry pustule increases round its circumference in a serpiginous manner; and Variola hamorrhagica, or black smallpox, a variety of the disease much to be dreaded from its terrible mortality. In some cases this form of the disease is so rapid in its course that the ordinary symptoms never become developed, but rapid collapse follows the abundant loss of blood from various mucous surfaces. In others, which are not quite so severe, abundant crops of petechiæ or even ecchymosis are ushered in by symptoms of 
fever, with great restlessness and prostration. In a third class of cases, after the appearance of an abundant eruption, perhaps confluent, with the vesicles full of pus, there appears suddenly, about the twelfth or fifteenth days, a livid colour which becomes bluish-grey or black, and the patient becomes rapidly comatose, and dies. In all such cases I have noticed a striking deviation from the ordinary relations of the pulse frequency to the amount of eruption and temperature, for whilst the latter has been raised from the first days of the disease two or three degrees, and the eruption in some of the children has been so abundant that hardly a spot of the skin was to be found uncovered, the pulse, from the onset of the attack till the occurrence of death, has not exceeded 84 to 88 . . In $V$. hamor. rhagica, besides the extravasation in the skin, and the hæmorrhages from mucous surfaces, there will be found on postmortem examination cerebral hæmorrhage and extravasation into the pleura, pericardium, meninges, \&c.

Occasionally a few isolated pustules with their contents mingled with blood may be noticed, but these need not afford ground for a diagnosis of hæmorrhagic smallpox.

As regards the number and size of the pustules great differences will be found in different cases; for in some they may not number more than a dozen or twenty in all, whilst in others it may be difficult to find any part of the skin which is not almost covered by them. The eruption is also very often found on the mucous surfaces, especially of the mouth and nose, œsophagus, larynx, trachea, bronchi, and conjunctiva, and in these positions it goes through the same changes, except crustation, as it does on the skin, and the implication of the mucous surfaces seems to be in proportion to the amount of the rash on the skin. In the mouth it gives rise to increased flow of saliva and to difficulty of swallowing, and in sucklings it prevents them taking the breast. When it attacks the air-passages it excites cough, hoarseness, and difficulty of breathing. The external ear is very often the seat of profuse eruption.

Suppuration of the cornea and perforation, with subsequent phthisis of the globe, is an occasional complication of smallpox, and seems to be the result of some metastatic process. Occlusion of the nostrils following the cicatrisation of the ulcers left by an abundant eruption is also sometimes seen. Croupo- 
diphtheritic inflammation of some or other of the mucous membranes is not an uncommon complication of smallpox, and scarlet fever and measles may be found, as already mentioncd, running a coincident course with variola in the same patient. We may also find our patients attacked by local inflammation during the course of smallpox, such as pleurisy, pneumonia, encephalitis, meningitis, arthritis, pericarditis, nephritis, \&c.

As sequelæ, I may mention furunculosis and phlegmonous inflammation from the absorption of the pustular contents, noma, necrosis of bone, obstinate anæmia, and the whole host of scrofulous diseases.

Causes.-Smallpox is an eminently contagious disease, the virus seeming to exist in the pustular matter and blood of the patient, being most powerful during the stages of eruption and decrustation, and it is probable that it obtains access by the mucous membranes. Whether the contagion can be carried by an intermediate person is not yet very clear, and any facts bearing on this point should be carefully sifted.

Different individuals seem to be susceptible to the virus of smallpox in very various degrees, and a curious fact about this disease is that it is communicated from the mother to the fœtus, sometines destroying the life of the latter.

Prognosis. - This must be influenced in great measure by the size of the child, the severity of the disease, its complications, and the surrounding conditions, and also the time which has elapsed since the child was vaccinated. My own experience, and the statistics of cases observed in a very severe and protracted epidemic, place the value of vaccination beyond a doubt. Of $4 \% 0$ children suffering from smallpox who were under my care, 315 had not been vaccinated and 208 died, or 66.03 per cent.; whilst of the 105 who had been vaccinated only 14 died, or 13.3 per cent. Amongst the vaccinated children the course of the disease was usually mild, favorable, and short, whilst in the unvaccinated it was generally scvere, and often presented the confluent and hæmorrhagic varieties. Again, in the former class the greatest mortality occurred in the first and second years, the children under one year dying almost without exception; whilst amongst the vaccinated the result was entirely otherwise, for of those who died two were in the second year, two in the third, one in the fourth, two in the 
fifth, one in the seventh, three in the ninth, and one each in the tenth, eleventh, and twelfth years. The prognosis must always be more unfavorable the younger the child, and especially so if the child is at the breast; and a child already suffering from some other disease readily succumbs to an attack of smallpox. All inflammatory complications, especially if of a diphtheritic or pyæmic nature, increase the dangers of smallpox; and when the disease is of the hæmorrhagic type the termination is almost inevitably fatal. Sudden recession of the pustules, accompanied by a yellowish or greyish-blue discoloration of the skin, is also to be looked upon as an indication for an unfavorable prognosis.

In very severe cases it is at first somewhat difficult to recognise the disease in the prodromal stage, and it is quite possible to mistake it for typhoid or measles, especially for morbilli papulosi ; but the enlargement of the spleen and other symptoms of typhoid, and the catarrhal affections peculiar to measles, will soon make the diagnosis clear. Slight cases of smallpox sometimes resemble such skin diseases as acute eczema, scabies, or syphilitic papulæ, but a little caution will readily eliminate such difficulties.

Treatment.-We have no specific for the treatment of smallpox, but the efficacy of vaccination as a prophylactic is beyond dispute. As a further precaution, all cases of the disease ought to be rigidly isolated. Numerous remedies have been recommended as having some influence in shortening the disease or in mitigating the eruption, but I know of none which possesses any such influence. In addition to rational dietetic precautions our treatment can be only symptomatic, and directed against the many risks and complications which may befall the patient. Quinine, digitalis, and the mineral acids may be found serviceable in reducing the temperature; cold baths or packing, or the inunction with lard, will be found grateful by relieving the feeling of burning in the skin; and when the mouth is affected a lotion of chlorate of potash will be found useful. The hæmorrhagic disease may be treated with quinine and stimulants. When the eyes are much affected little can be done beyond keeping the eyelids clean, on account of the great swelling of the eyelids.

When the disease is mild therapeutics are not required. 


\section{Varicella, chicken-pox}

This is an acute, epidemic, and contagious disease, marked by an eruption very much resembling that of smallpox, though the diseases are essentially different.

The period of incubation seems to vary from eight to fourteen days, the majority of the cases having no perceptible prodromal stage, and where this is observed it is very short and very mild, marked only by slight variations of temperature, signs of general discomfort, and scme pains in the limbs.

The eruption always appears quickly, so that its complete development does not occupy more than a few hours. It consists of a number of vesičles. more or less abundantly scattered over the skin, varying from the size of a millet to a hemp seed tightly distended, and pearly in appearance, surrounded by a reddened margin, and containing clear or slightly turbid serum. In exceptional cases the vesicles are larger in size, present an umbilicoid depression, and have purulent contents.

The duration of the eruption of chicken-pox is very short, seldom exceeding three days, the vesicles drying up into small yellowish or brownish-yellow scabs, which fall off in a few days and leave small scars behind, which generally disappear after a time, but which sometimes leave permancnt marks. The eruption usually appears first on the trunk, especially on the back, and rapidly extends over the extremities, face, and even the hairy scalp; and it does not always come all out at once, but may appear in successive crops, so that whilst some of the vesicles may be seen in the stage of crustation, at the same time others may be seen quite fresh.

The increase of temperature throughout this discase is seldom of any importance, rarely exceeding $38.5^{\circ}$, and it differs from the temperature of smallpox in that the rise begins with the appearance of the rash or a short time before, and the maximum is reached when the largest number of fresh vesicles is out. With a fresh crop of eruption the temperature may rise again, and the defervescence is always rapid. Complications and sequela are unknown in this disease, and the result is always favorable. The period of its most frequent occur: rence is from the second to the seventh ycar, but it may be 
seen in children of all ages and all classes of life. It is undoubtedly a contagious and transmissible disease, and I have repeatedly communicated it by inoculation, the eruption usually in such cases appearing over the whole surface about the eighth day. It confers no immunity from attacks of smallpox, for they are often seen following one another; nor does vaccination seem to afford protection from varicella, and the same patient may suffer several times from this disease.

There is little treatment required beyond keeping the patient in bed and giving some slight laxative.

\section{Vaccina and Vaccination}

Vaccination, since its establishment by Jenner in 1796, has been adopted in most civilised countries, and though it cannot be said to confer absolute immunity from smallpox, yet it is established beyond doubt that it has diminished the intensity of the variolous poison to an enormous extent, is able to modify the course of the disease, and has very largely diminished the mortality.

Vaccination may be done with lymph direct from the udder of the cow (primary lymph), with lymph which has passed through a human patient (humanised lymph), or with lymph which is the result of vaccination of the heifer with lymph from the human sulject (retro-vaccination lymph). The best method of vaccination is undoubtedly from arm to arm, as lymph preserved in any kind of way seems to lose its activity after awhile, and from three to six points of vaccination should be made on the outer side of the upper arm. Two or three days after a slight swelling should be visible, and by the fifth or sixth day the vesicles should be apparent, and reach their greatest size by the eighth or ninth, when they are surrounded by a very well-marked ring of inflammation. The contents of the vesicles subsequently become purulent and dry into a scab, which drops off about the eighteenth or twentieth day, leaving behind an irregular cicatrix, which gradually becomes" of a milk-white colour, and remains as a permanent mark through life.

As a rule there is very little general disturbance during the course of vaccination, though the temperature may rise slightly 
between the eighth and twelfth days, and there may be some evidence of feverishness and discomfort. 'There is often a good deal of burning and itching of the pustules, which may be much relieved by anointing with oil. Care should be taken that the heads are not rubbed off.

The best age for vaccinating children is between the fourth and twelfth months, and it should be done only when they are in good health. Children suffering from bronchitis or pulmonary catarrh should not be vaccinated, as it seems to excite slight pneumonia under such circumstances. It is betterdone during the summer months, as at that time it seems to take more readily and the pustules develop better.

Sometimes we have erysipelas or erythema resulting from vaccination, the former spreading from the seat of vaccination, and often proving very troublesome and sometimes even fatal. The erythema is generally fugitive, and not of much consequence. An eruption, vaccinola, resembling varicella, is sometimes seen to follow soon after vaccination; and in scrofulous children vaccination occasionally seems to excite eczematous and impetiginous eruptions of an extremely troublesome kind. In some cases the pustulation seems to occur doubly, so that from the six vaccine insertions twelve vesicles arise, in others repeated vaccinations have no effect whatever, and in a few their appearance seems curiously delayed.

Much has been said about the communication of other diseases by the agency of the vaccine virus; but it is not known to take place in the case of any but syphilis, and then only when the lyınph is mixed with the blood of the affected child, and this can be avoided by a little careful inquiry into the history and appearance of the child from whom the matter is taken, and by always acting on the rule of never vaccinating from a child under three months old. Of 12,000 vaccinations we have performed in the Prague Hospital no such case of infection is known. If the vaccine pustule should take on a chancrous appearance, then it may be that the child is syphilitic and the vaccination has only induced a local indication of it, or it may be that the disease has really been communicated by the virusa possibility which should be borne in mind.

A number of opponents of vaccination have arisen who assert that since its introduction the human constitution 
has been weakened, and that scrofula, tubercle, and even mental disease have become more common; but they do this either from want of experience, intentional perversion, or from simply repeating what they have heard from others, without being able to adduce any facts in support of it.

\section{Typhoid Fever, Typhus abdominalis ileotyphus}

This is a common disease in children, for in the Prague Hospital out of 80,245 patients under treatment during ten years, 1180 were cases of typhoid. The exanthematic typhus fever is a much less common disease. It may be stated generally of typhoid that it is a much milder and less fatal disease amongst children than amongst adults.

Anatomy.-The most important changes occur in the intestinal canal and its follicular apparatus, the earliest appearances consisting of a more or less extensive swelling and reddening of the mucous membrane, with shedding of the epithelium and an especially characteristic enlargement of the solitary follicles and of Peyer's patches, the former appearing as greyish nodules of pin-point size, surrounded by a reddish margin, and the latter as broadish elevations, about an inch to an inch and a quarter in length. The cellular proliferation is found to affect the follicles only, the intervening tissue being left unimplicated, and by the necrotic destruction of the follicles there results the characteristic typhoid ulceration. This lesion is not so universal as is generally supposed, for of twenty post-mortem examinations of patients who had died of typhoid I found it absent in four. It is usually situated in the neighbourhood of the ileocæcal valve, the ulcers being superficial, shallow, and occasionally found to be already in process of cicatrisation, whilst the occurrence of perforation is not common, as 1 have met with it only twice. 'The mesenteric glands are swollen, the increase in size being most marked in the neighbourhood of the follicles which have undergone the greatest changes; and this enlargement may result in cheesy degeneration. Coincident with these changes in the intestines there is always an enlargement of the spleen, the pulp being very rich in blood, and very easily lacerated. The blood is found to be thin and fluid, and there 
is generally some hyperæmia of some of the organs, such as the brain, the lower lobes of the lungs and the kidneys.

Symptoms and Course.-The onset of the disease is seldon sharply marked, but is generally indicated by an insidious sense of lassitude, accompanied by headache, loss of appetite, disturbed sleep, occasionally epistaxis, repeated vomiting, diarrhœa, shivering, and exaltation of temperature.

The temperature curve is very characteristic, and is of great importance both for diagnosis and prognosis. After the initial rigors it usually takes the form of a "rising zig-zag" (Wunderlich); that is, it rises about one degree from morning to evening, and falls about half a degree between evening and morning, until at the end of the first week or the beginning of the second it reaches from $39.5^{\circ}$ to $40.5^{?}$. About the tenth or twelfth day the morning and evening remissions become more marked, and towards the end of the second or beginning of the third week the fall of both morning and evening becomes relatively greater, and the temperature gradually regains its normal condition. The maximum temperature, $40^{\circ}$ to $41^{\circ}$, occurs from the seventh to the twelfth day, according to the severity of the case, but the occurrence of complications may alter or completely obliteratc the above-mentioned characters of the temperature curve. According to Gerhardt the temperature and pulse-curves have an average relation which is influenced by age, so that above ten years of age the pulse-curve is under the temperature curve, so that a temperature of $38^{\circ}$ and $39^{\circ}$ will correspond respectively to pulses of $90-100$ and $110-120$; whilst under six years of age similar temperatures will give pulse frequencies of $120-$ 140. 'The pulse has a feebler beat in typhoid than in inflammatory diseases, and the dicrotic pulse is not seen in young children.

The nervous lesions seen in this disease seldom reach the intensity which they often present in adults, and are chiefly evinced by muscular weakness, restless sleep, delirium, and difficulty of hearing.

The most important local symptoms occur in the digestive organs. The abdomen is distended, and is tender on pressure, especially over the spleen and cæcum, pressure over the latter region being always accompanied by a gurgling perceptible to the fingers, and due to the presence of flatus in the neighbour- 
hood of the ulceration. This sign is not so well marked in children as in adults. Diarrhœa does not usually set in early in the disease, but begins in the second week, the stools being fiequent, thin, of a bright brown-yellowish colour, very much resembling pea-soup, of a disagreeable odour, having an alkaline reaction, and containing salts, fat, the detritus of food, and a quantity of epithelium.

In some cases, and, indeed, in every instance in one particular epidemic noticed by myself, there was no diarrhœa, but, on the contrary, marked constipation, which actually required the use of laxatives to overcome.

Hæmorrhage from the intestines, and perforation of their coats, are exceptional occurrences in children.

The tongue is more or less coated, and of a bright red colour at the point and edges, with the papillæ increased in size. In very severe cases it is dry, fissured, and covered with sordes, which is also to be noticed on the teeth and lips.

The appetite, as a rule, disappears entirely, whilst the thirst becomes very troublesome, and vomiting is a frequent symptom in the early stage of the disease.

The spleen is always swollen, being incrcased to two or three times its normal size, though in children this is a condition which cannot always be ascertained from the smallness of the organs, but there is always tenderness over the spleen, and pressure on it will always rouse the patient from the deepest sleep.

'The roseoloid rash, which is so constantly seen in adults, is by no means so frequent in children. It appears generally about the beginning of the second week, and consists of isolated spots, varying from pin-point size to that of a linseed, situated chiefly on the skin of the abdomen, rarely on the breast and extremities, and of a peculiar rose-red colour. The spots last from three to six days, fading gradually away, and-are succeeded by a fresh lot; any fresh typhoid localisation in the intestine being accompanied by a fresh outcrop of the roseoloid spots.

Miliaria crystallina is an eruption of small pearly vesicles which appear in great numbers over the abdomen and thorax, usually during the third week of the disease. It has a favorable indication, and after the vesicles burst there is usually 
some desquamation. Sometimes we see petechiæ, either alone or in association with the roseoloid rash, but they do not seem to have the unfavorable indications which might be expected, though they generally occur in children previously diseased, or existing under unfavorable sanitary conditions. Herpetic eruptions on the lip, chin, and forehead are sometimes to be noticed. - Bedsores are apt to form in children as in adults, unless care be taken to prevent them, their usual seat being over the sacrum. Noma also sometimes attacks the face, and genitals in girls; and in a few cases $I$ have seen as a sequela furunculosis of a severe and even dangerous character, in one case there having been as many as thirty large furunculi.

Catarrh of the respiratory mucous membrane is of frequent occurrence in typhoid, and pneumonia of hypostatic origin, œdema of the lungs, and even pulmonary gangrene may have to be contended with. In several cases I have seen acute pulmonary tuberculosis occur immediately after convalescence from typhoid, in children who have come of scrofulous and tubercular families; and in such cases I have always found, on post-mortem examination, old cheesy or tubercular centres. Extensive thrombosis of the pulmonary arteries has also been found as another complication of typhoid.

Ulcers in the larynx have been met with in some cases, their symptoms appearing about the third week of the disease, and consisting of hoarseness, loss of voice, and pain in the region of the larynx. In one case I saw perforation of the larynx by this ulceration, and the occurrence of emphysema over the chest and back, resulting in death. These ulcers may lead to perichondritis and necrosis of the cartilages.

Croupous laryngitis may also occur during the course of typhoid, and in this disease no laryngeal symptoms should be carelessly regarded.

Towards the crisis of the disease the urine becomes diminished in quantity, of a dark brownish-red colour, of acid reaction, with a diminution of the chlorides, and increase of the uric acid, and there may be a trace or even an abundance of albumen. In the case of a boy who had suffered from croupous cystitis during the course of typhoid I found shreds of croupous membrane passed in the urine. Croupous diseases of the mucous membranes may be seen in typhoid, and also purulent 
pleuritis, nephritis parenchymatosa, parotitis, thrombosis of arteries and veins, and hydrocephalus. Cases of the lastnamed complication have been noticed by Löschner and myself as occurring in children between two and four years of age suffering from well-marked symptoms of both diseases.

Of the sequelæ of typhoid may be mentioned aphasia, mental weakness or even absolute idiocy, these conditions usually disappearing in a few weeks, having originated apparently in anæmia or some other lesion to the circulatory apparatus of the brain. Rilliet and Barthez have also observed chorea with softening of the spinal cord.

The so-called relapses of typhoid fever do not often occur in children, but sometimes we see something resembling them after the feverish symptoms have completely disappeared, the subjective symptoms have ameliorated, an apparent crisis occurred, and for three or four days convalescence seemed to have been advancing, when there may suddenly. occur all the old symptoms-fever, delirium, eruption, and diarrhœa-and for four or six days the case looks as bad as ever. This remission may occur two or three times, and protract the duration of the illness very considerably.

Causes.-This is not a disease commonly seen in very early life, though some observers (Bednar, Bühl, Löschner) have recorded its occurrence in children a few weeks or even a few days old. It attacks children most commonly between the ages of five and twelve, and boys seem to suffer more frequently than girls. The time of year of its greatest intensity is late in summer and during autumn. It is now almost certain that it is not contagious, no case in the least supporting the contrary view having come under my own notice during my fifteen years' experience in hospital and private practice, the cases where its direct source could be traced all indicating origins of another kind. The most frequent of these is undoubtedly impure drinking water, a careful investigation having, in innumerable instances, referred an endemic of typhoid fever to this source. In one instance I found that the use of spring water, which had become contaminated with sewage, produced in the short space of three days severe typhoid in ten members of one family; and in another house, when the same water was used, about thirty people were attacked in a few weeks. The obser- 
vations of Buhl have abundantly shown that there is a close connection between typhoid fever and the use of surface water. Other excitants, or, at least, predisposing causes, are to be found in unhealthy, damp, and easily flooded dwellings, and irregular ways of living. 'There is every reason to believe that the origin of typhoid poison is to be found in decomposing animal matter, and it has been stated also to be due to vegetable parasites - the rhicopus nigricans and the penicillium crustaceum.

Diagnosis. - The chief objective symptoms, as already mentioned, are the peculiar temperature curve, the enlargement of the spleen, meteorism, diarrhœa, and the rash. The disease most likely to be mistaken for typhoid is miliary tuberculosis, and in order to discriminate between them it is necessary carefully to consider the history of the patient and the course of the temperature, and the facts that in acute tuberculosis there is a peculiar increase of the sensibility of the skin and an absence of cruption. Meningitis and acute hydrocephalus also present symptoms which may lead to their being mistaken for typhoid, and when the latter occurs coincidently with hydrocephalus, the head symptoms are likely, by their prominence, to conceal the existence of the typhoid fever.

Gastric and intestinal catarrh, with feverish symptoms, can scarcely be mistaken for typhoid in their early stages, and later on they may be readily discriminated by an absence of the peculiarities of temperature, of the swelling of the spleen, and of the eruption. The same may be said of the so-called cerebral pueumonia already spoken of, though there may be some difficulty in a case of typhoid where there is also pneumonia.

Prognosis.-This is always much more favorable in children than in adults, and in giving an opinion as to the probable result of every case attention must be given chiefly to the indication of fever, to the abdominal symptoms, to the presence of any complications, and to the previous history of the patient. The average mortality of the disease in children seems to be one in ten or twelve, and of the cases in one hospital it has been one in thirteen.

Treatment. - To prevent this disease great care should always be taken to procure a pure and untainted water supply. Its actual treatment can be only expectant and 
symptomatic, for we have no specific for it, despite the abortive treatment by calomel recommended by Franke, Wunderlich, and others. The patient should, if possible, be placed in a cool and well-ventilated chamber, and all slops and evacuations should be at once remored and disinfected. The diet should consist of strong soup, milk, eggs, and fresh cold water may be given freely as a drink, either alone or acidulated with muriatic acid.

The cold-water treatment is much in use now for typhoid fever, though for children its use may not be altogether free from risk. According to Brand's method the patient is wrapped in cold-packing as often as the temperature in the axilla exceeds $39.5^{\circ}$, or placed in a bath of $23^{\circ}$ to $26^{\circ}$, and whilst there douched with water at $10^{\circ}$ to $16^{\circ}$. Cold water is given to drink every quarter of an hour, and at intervals of three hours beef tea or milk is given. For slight cases of fever this treatment involves really too much trouble to the attendants, and less elaborate measures suffice; but in severe cases it is especially suitable, though I cannot but think that it must have a tendency to excite bronchitis and pneumonia, and a depressing influence on weakly and anæmic children. In severe cases of typhoid, especially in weakly children,. stimulants must be liberally administered. Quinine should also be given and Dover's powder for the diarrhœa, starch and opium clysters being also used for the relief of the meteorism. If there should be hæmorrhage frcm the bowels, the pernitrate of iron may be given with advantage. Any complications, such as bronchitis or pneumonia, must be treated on the principles already enunciated in connection with these diseases. Retention of urine must be relieved by regular and careful use of the catheter. When the fever has disappeared and the tongue has become moist and clean, tonics may be given, especially the preparations of cinchona, and the diet should be gradually and cautiously altered to more solid food as is used in health. For the prevention of bedsores there is nothing like a water-bed.

\section{Relapsing fever, Febris recurrens}

This disease, which was first made known in Germany by Griesinger, is an epidemic and contagious disease occurring 
chiefly amongst the poor, or people suffering from starvation and living in bad hygienic conditions.

Anatomy.-The muscles are found to be firm and of a dark colour, the spleen much enlarged, sometimes to as much as three or four times its normal size, the liver being also similarly affected, and both organs are often scattered with numerous miliary abscesses. The muscular tissue of the heart and the renal epithelium are found to have undergone fatty degeneration, and ecchymoses are often seen in various organs. The mesenteric glands are usually enlarged, with indications of intestinal catarrh, and occasionally such other lesions as bronchitis and pneumonia.

Symptoms and course.-The period of incubation of this disease seems to average about six or eight days, and the initial symptoms consist of headache, lassitude, muscular weakness, and pains in the limbs. The disease is usually ushered in suddenly by rigors, an exacerbation of the initial symptoms, the pains in the limbs somewhat resembling those of rheumatism, and occasionally there is vomiting.

In addition to these the temperature rises to $39.5^{\circ}$ or $40^{\circ}$ and the pulse to 140 or 160 , being strong, and often dicrotic. During the fever the skin is usually moist or even perspiring. After these symptoms have lasted five or six days, the temperature rising the while sometimes as high as $42^{\circ}$, a sudden remission takes place, and in a single day the temperature may fall to $35^{\circ}$ or $36^{\circ}$, and the pulse to $60^{\circ}$ or $70^{\circ}$, and the disease for six or eight days may seem to have disappeared completely, and this indeed is sometimes the case, though much more frequently fresh rigors usher in the old symptoms again and these disappear a second time after a few days' duration. Sometimes there is a third attack, though this is not the rule. During the fever the urine is scant and the chlorides diminished in quantity, and albumen and tube-casts are frequently to be found. Herpetic eruptions on the face and miliary eruptions on the trunk are often seen in this disease, and sometimes the patients suffer from jaundice and biliary symptoms (from fatty degeneration of the liver), producing the "bilious typhoid" of Griesinger.

Convalescence is usually prolonged, and we occasionally see such sequelæ as anæmia, enlargement of the spleen, and abscesses 
of the skin. The prognosis is generally favorable, as the mortality does not seem to be more than three per cent.

Treatment.-It is a disease which very urgently demands prophylactic isolation, and its general treatment can be only expectant and symptomatic. The sick-room should be airy and well ventilated, absolute rest should be maintained, and the diet should be mild, nutritious and regular. The most useful drugs will be found to be quinine and stimulants, and the coldwater packing may be of use in reducing the temperature.

\section{Intermittent Fever; Ague}

This is a non-contagious disease, of endemic occurrence, having its origin in malarial poison, and it pursues in children the same courses which mark its existence in adults.

We are yet unacquainted with the nature of this malarial poison, and know little more about it than that its development is favoured by the existence of marshes or other conditions by which surface water is pent up, and that there are certain conditions of the individual by which its influence is favoured. It has been supposed to be a vegetable parasite.

It has been established by Stokes that intermittent fever may be communicated from the mother to the foetus, and in support of this I may mention a case which came under my own notice, in which a woman who had suffered from intermittent fever during pregnancy noticed during the attack a peculiarly strong movement of the fotus. She had twins, and they were found at birth both to present enlargement of the spleen. Boudin and others have stated that intermittent fevers can be communicated to the child by the mother's milk, but I have noticed no facts in support of this.

The anatomical changes produced in children by the malarial poison are not usually so well marked as in adults, but they are essentially of the same nature. The most important is the simple or acute enlargement of the spleen, which leads in the chronic stage to fibroid degeneration, amyloid degeneration, or pigmentation of the organ. Pigment also becomes deposited in the liver, kidneys, lungs, intestinal canal and lungs; and we may also say pigmentary embolism and apoplexies. Amyloid 
degeneration of the kidneys, parenchymatous nephritis, dropsy, sudamina, and anæmia, may also be ranked amongst the results of this disease.

Symptoms and course.-The younger the child the less completely and regularly do the peculiar paroxysms occur. The quotidian type of the disease, or that in which the attacks occur daily, is the most common amongst little children, whilst in. those more advanced we see the tertian (attack every fortyeight hours), the quartan, and the duplex intermittent fever. In children the attacks are very seldom complete, and do not recur with absolute regularity, the first stage, or that of rigor, being often completely absent, and in others only very slightly marked by a sense of cold and blanching of the skin, lasting from a few minutes to abbut an hour. The hot stage is always well marked, even at the beginning of the disease, lasting from two to four hours, and it is often the only indication of the disease. The face becomes markedly reddened, and even slightly swollen; the patient becomes thirsty and restless, and the temperature rises to $40^{\circ}$ or $41.5^{\circ}$.

The sweating-stage is, on the whole, shorter and not so pronounced as in adults, being in some cases almost entirely absent, and in others indicated only by a transient moistening of the skin.

In some children the enlargement of the spleen is the only indication of the disease; at least, it is a sign which is never absent, and it is most marked during the attacks. I have examined fifty children in a village where ague was endemic, and in every one $I$ found the spleen enlarged, although about two thirds of the children were regarded as healthy, and had never been known to suffer from any aguish attack. In districts where intermittent fevers are rife, cases often occur when the disease is marked, but these are rclatively much less frequent in children than in adults.

The diagnosis of this discase is very easy when its features are well pronounced, but it is sometimes rendered obscure by reason of irregularities in the attacks and gencral symptoms. Almost any disease may occasionally take on intermittent characters, and this must always be remembered; and, on the other hand, I have repeatedly found physicians diagnosing all sorts of inflammatory diseases when they have happened to see 
the patients in the hot stage, when, after waiting a day or two, a recurrence of the attack showed it to be malarious.

Especial care must be taken in the cases of teething children, who are so apt to suffer from recurrent feverish attacks, not due to ague.

Treatment.-Children suffering from symptoms of malaria ought, if possible, to be removed at once from the infecting district; or if this cannot be done, they should be placed in as good hygienic condition as the circumstances will allow, and be especially protected from the influence of cold and the night air. The best remedy is, of course, quinine given in large doses, one to three grains for a dose, according to the age of the child, and it ought to be given during the intermission. If it cannot be borne by the mouth, it may be given by enema in larger doses, by subcutaneous injection, or by inunction. If quinine have no effect, arsenic may be tried, in the form of Fowler's solution, in doses of one or two drops daily and increased gradually. I have often seen advantage, especially at the beginning of the treatment, from a combination of quinine and opium with arsenic. The treatment must always be continued until all variations of temperature have disappeared, and till the spleen is diminished in size. For the cachexia which sometimes remains, change of air and diet, and quinine are the: best remedies.

\section{Cholera Asiatica}

This is a disease of an acute miasmatic and contagious character, excited and spread by some poison not yet discovered.

The post-mortem appearances of this mysterious disease consist of extreme post-mortem rigidity, a bluish lead colour and collapsed condition of the skin, thickening of the blood with a diminution of the salts, especially of the chloride of sodium, whilst the phosphates and potash salts are increased, distension of the stomach and coating of its mucous surface with thick mucus, congestion of the mucous coat of the intestines, and destruction of the epithelium and swelling of Peyer's patches.

Klob has discovered confervoid growth in the intestine and in the evacuations. Other conditions are also found with more or less constancy, such as hyperæmia of the kidneys, unusual 
dryness and blanching of the muscles, liver, and lungs, and a peculiar soapy feeling of the serous membranes, especially of the arachnoid and pericardium, which has been described by Löschner.

Symptoms and course.-In some cases the disease is preceded by certain prodromal symptoms, much derangement of digestion, dyspepsia, diarrhoa, and a general sense of malaise. The milder forms are termed cholerine, and in them it scems modified either by the reception of a smaller amount of the poison, or by a greater power of resistance on the part of the patient. The onset of the disease is always marked by diarrhoea and vomiting, restlessness and pain over the stomach; the vomiting consisting at first of the food and drink which have been taken, either unaltered or half digested; but afterwards, as the disease progresses, it has the characteristic rice-water appearance and is mixed with flocculent mucus.

The diarrhœa, which is the most constant and usually the first symptom, is accompanied by severe abdominal pain and considerable meteorism.

The stools are passed in a quick violent stream, and very frequently; they are watery, and deficient in biliary colouring matter, or at first of a pale yellow colour, and afterwards resembling rice water. Occasionally they are mixed with blood and resemble plum juice, and they may be either free from smell or possess a very offensive odour. Their reaction is markedly alkaline, and only become acid during the stage of reaction, when they become pultaceous. During the algide stage they consist of 96 or 98 per cent. of water and chloride of sodium, with some albumen occasionally and carbonate of ammonia. Monti states that in the choleraic evacuations from infants at the breast he has obtained distinct evidence of bilifein, and in more advanced children the addition of nitric acid gives a red colour.

At the beginning of the disease the abdomen is somewhat distended, but as the evacuations become profuse it becomes retracted, and sometimes the convolutions of the intestines can be traced through the parietes. During the algide stage the tongue may be moist and of a greyish-yellow colour, or dry and red, the edges sometimes being of a bluish tinge; but in the asphyctic stage it is dry and viscous. Hiccup is stated by 
Löschner to be a frequent symptom in children, whilst Monti regards its occurrence as rare. During the last epidemic I noticed pretty frequently thirst is always a troublesome symptom, and when the evacuations are profuse it is often very aistressing.

The course of the disease may be conveniently divided into the algide, asphyctic, and reactive stages, and during each of these the conditions of the circulation and respiration undergo alteration. During the first stage, the heart's action is tumultuous and becomes gradually weaker and out of rhythm; and its strength is not found to be in proportion to the strength of the pulse (Löschner, Monti), the latter being at first frequent, and gradually becoming small and thread-like, and finally' not to be felt. During the stage of reaction the pulse becomes again frequent and strong, and in the subsequent typhoid stage it is sometimes retarded. The veins are fillerl with dark bilberry coloured blood, giving rise to cyanosis which may be either general, producing a deep blue speckling of the skin which must always be regarded as an unfavorable sign, or it may be partial and affect only the lips, cheeks, eyelids, ears, fingers, or toes. In children previously diseased or anæmic the cyanosis is represented by a bluish-grey earthy corpse-like colour of the skin. After the establishment of the cyanosis further signs of collapse set in, the temperature sinking to $31^{\circ}, 30^{\circ}$, or even $29^{\circ}$, the skin losing its sensibility and elasticity, and being covered with a clammy sweat. The eyelids are half drawn over the deeply sunk eyes, the cheeks and lips shrunken, the nose pinced, and the unclosed fontanelles retracted. The cyanosis and the indications of collapse seem to have a close relation to the severity of the diarrhœa.

The respiration is generally accelerated, sometimes to as much as 60 or 70 in the minute, and may be irregular and interrupted by deep sighing. 'The respired air is generally cold, as may readily be ascertained by holding the hand over the patient's mouth. Whether these changes are the result of lesions in the respiratory organs or in the nervous system is not yet quite clear.

The vox cholerica, so well marked in adults, is not often observed in children, though in them the voice may be found weakened and husky. 
The nervous symptoms which are met with in children suffering from cholera include sopor, delirium, convulsions, and the peculiar muscular cramps and rigidity seen also in adults, but more frequently in children.

The quantity of urine is generally diminished during an attack of cholera, and sometimes there is complete suppression, in which case the last which is passed generally contains albumen and some fibrinous casts. If recovery takes place the secretion becomes re-established, and this may take place either suddenly or gradually, the access of the convalescence being commensurate with the rapidity of the return of the renal function. During the stage of reaction the urine is always acid and of low specific gravity, and it generally contains albumen with a diminished amouht of chlorides. A diminution of the amounts of other secretions, as of the conjunctiva, lachrymal and salivary glands, may also be noticed in cholera.

Sometimes the duration of the disease is very short, not more than twelve to eighteen hours, though in the majority of the cases it lasts several days. Löschner has divided cases according to the character of their courses into the three classes of rapid, continuous, and remitting.

The disease may end in death, recovery, or in the choleratyphoid. The greatest mortality arnongst children, according to Monti, is between one and five years of age, and death occurs most commonly during the asphyctic stage, and not often during the algide condition. The younger the patient the greater the risk of an unfavorable issue, and the greatest mortality always occurs in the early part of an epidemic. According to Löschner, 121 children died out of 235 ; to Hervieux, 70 out of 117 ; to Monti, 38 out 54 ; and on an average we may say that the mortality is about fifty per cent.

Cholera-typhoid is a condition which seems to be the result of uræmia, strong reactive fever, anæmia of the brain, \&c.; and its symptoms include sopor, somnolence, delirium, convulsions, dryness of the mucous membranes, vomiting, diarrhœa, a more or less exalted temperature with nocturnal exacerbations, strong and often retarded pulse, injection of the conjunctiva, muddiness and superficial ulceration of the cornea, dropsy, and œdema, and occasionally an eruption resembling urticaria.

Causes.-The specific cause of cholera is yet unknown, but 
the method of its conveyance is in all probability the contamination of drinking water by the evacuations. Children of all ages, even sucklings, are attacked by cholera, and it spares the healthy no more than the weakly and ill-nourished, though there can be no doubt that bad food, exposure to cold, and living in wretched dwellings, act as strong predisposing causes.

Treatment.-In this, as in all other contagious and infectious diseases, isolation as stringent as possible should be put in force, and all the evacuations and fomites should be disinfected. Sucklings should be weaned, if it be possible, to avoid it whilst cholera is raging, and, especially, care should be taken of children who are teething.

Out of the large number of drugs and methods of treatment which have been recommended for cholera not one has yet proved of specific value, and all our efforts must therefore be directed against the various symptoms as they appear.

During the presence of an epidemic of cholera the diet of children should be carefully regulated, so that any kind of food should be avoided, such as some kinds of fruit, which may have a tendency to produce diarrhœa, and any looseness of the bowels should be at once attended to by keeping the patient in bed and administering mild opiate clysters.

In case of an attack of cholera some strong black coffee or tea with rum in it should be given, the patient should be placed in a hot bath for five or six minutes and wrapped in hot blankets, to induce perspiration. I have often seen profuse diaphoresis follow the prolonged use of wet packing. For the diarrhœa, opiates, the mineral acids, and aromatics will be found best, though it must be confessed that opiates, like everything else in this disease often fail us. An enema of starch and opium will often give relief and check the diarrhœa when the opiate is rejected by the mouth. When cyanosis and indications of collapse set in, stimulants ought to be freely given, the patient should be placed in a bath at $39^{\circ}$ to $42^{\circ}$, the skin rubbed with some stimulant embrocation, and stimulants given internally, such as camphor, musk, and spirits.

For the relief of the vomiting ice or iced soda-water may be given ; and Monti had strongly recommended creasote dissolved in a little ether and mixed with honey and peppermint-water: Hypodermic injection of morphia has also been recomnended, 
but I have not used it much, for morphia is always a dangerous drug for children.

The muscular cramps may be very much relieved by rubbing the affected limbs with a liniment containing chloroform or some other sedative. When reaction sets in the stimulant should be gradually withdrawn, and the natural efforts at recovery should not be hastily interfered with.

In cholera-typhoid, also, the treatment must be symptomatic. Sponging the skin with vinegar and water will be found to restore the temperature, cold applications to the head may mitigate delirium, and quinine with acetate of potash may be of assistance in relieving the symptoms of uræmia. During convalescence great care in the diet must be taken.

\section{Syphilis}

In children this disease is of course met with in its acquired forms very exceptionally, but in its heriditary varieties it is only sadly too common. It is very often communicated from the mother to the fotus, and in this way is frequently the cause of premature death of the child, followed by miscarriage ; or it may live till the full time and be born with marks of the disease, only to die an early and miserable death. In a third class of cases the child may appear quite healthy at birth, and the indications of disease may not show themselves until it is some weeks or months old.

Symptoms and course.-The phenomena of hereditary syphilis resemble very much the forms of secondary syphilis in the adult with reference to their seats; anatomical appearance, and combinations. The most frequent seat is the skin, the affected parts presenting a dirty greyish-white, faded and wrinkled appearance, very different from that of normal healthy skin. The palms of the hands and the soles of the feet are covered with thin, dry and glistening cuticle, easily cracked, and the skin of the forehead has a peculiar waxy and glistening appearance. As in adults, syphilitic skin diseases in children seem to have certain favourite seats, though they may occur on any part of the cutaneous surface, and they also have the same chronic course, the same tendency to relapse, and the same 
freedom from pain or itching; but the peculiar copper colour is not so well marked. The following varieties are met with:

Syphilis cutanea maculosa vel erythematosa, or roseola, consists of brownish-red spots varying in size from that of a linseed to a threepenny piece, spread in varying numbers over the trunk and extremities, and not commonly seen on the neck and face. These spots disappear after a brief existence and leave behind them light brown discolorations.

S. cutanea papulosa papula, or lichen, is marked by hard nodules of pin-point size, either scattered or gathered in groups over the trunk and extremities, especially on the palms of the hands and soles of the feet. After a while the nodules become marked by a slight desquamation, and sometimes form small ulcers.

S. cutanea squamosa, psoriasis syphilitica, may be developed out of the preceding eruptions or may be primary. It consists of brownish or dirty red spots, which desquamate in the centre, and healing there, spread towards their circumference in a serpiginous manner.

S. cutanea vegetans, condylomata lata, placques muqueuses, are nodular infiltrations of a dirty red colour, with superficial ulceration of the epidermis. They may be isolated or situated in groups, becoming confluent, and their commonest seats are the skin and mucous membrane of the genitals, anus, lips and nostrils, and in the folds of the skin, the condylomata spreading then by contact of the opposing surfaces. When the affected children are neglected and the condylomata not kept clean, they often give rise to very deep and extensive ulcerations and phlegmonous inflammation.

S. cutanea bullosa et pustulosa is a variety which generally occurs in the pemphigoid form in children, which is rare in adults, in whom it is seen as acne, varicelloid, and impetiginous eruptions; these latter, on the contrary, being rare in children. The pemphigoid bullæ may vary very considerably in number and size, and be isolated or confluent. They soon burst, and. leave ulcerating surfaces. They may occur on any part of the skin, but most frequently on the flexor surfaces of the hands and feet, and are almost always congenital, the affected child being generally atrophic, and seldom living longer than a few days or a week or two. Congenital pemphigus is not, however, always 
syphilitic, and it should not be accounted as such unless there be some other indication of the cachexia, for it may be of pyæmic origin.

The skin of syphilitic children is also liable to be attacked by cracks, ulcers, and abscesses, the latter being most frequently seen in the occipital scalp, in the neck and nates, and they are very slow to heal, their cicatrices being always pigmented.

The mucous membrane of the nose is an especially frequent seat of infantile syphilis, the peculiar "snuffles" (coryza syphilitica) being often the first indication of the constitutional disease. The child breathes with difficulty, and chiefly through the mouth, the nostrils being occupied by a muco-purulent discharge. In the more severe cases ozæna results, followed by necrosis of the bones and a falling in of the bridge of the nose.

Syphilitic children are frequently the subjects of obstinate nasal catarrh.

The mucous membrane of the throat is also the seat of syphilitic action in children, though not to the same destructive extent which is seen in adults, the affection being generally limited to reddening and swelling of the tissue, with a muco-purulent secretion, though in some cases it runs on to ulceration and even to destruction of the soft palate.

The lips of syphilitic children often look stretched, dry, and as if they were too short, and fissured at the angles, the cracks bleeding when the child cries. The mucous membranes of the mouth and tongue, of the intestinal tract, and of the genitals of girls, are the seats of mucous tubercles and ulceration.

The larynx is very rarely affected by syphilis in children, though I have seen specific ulcers in the larynx of a child eight days old, and I have also seen very well-marked stenosis of the trachea in a child, the result of syphilitic ulceration. Stenosis of the larynx is rare.

Förster and Schott have seen nodular infiltrations of Peyer's patches, and necrotic destruction of them; and otorrhœa and leucorrhœa are of frequent occurrence in syphilitic children.

Gummatous tumours are found in children, often at an early age, in such organs as the thymus gland, the liver, spleen, kidneys, and supra-renal bodies, lungs, tongue, and occasionally in the brain (Schott). Enlargement of the lymphatic glands is 
also very common. Syphilitic diseases of bone and periosteum and of the eye are not common in children, and they are rare in proportion to the youth of the patients. Paralysis due to syphilis will be met with occasionally in children.

I am not in a position to confirm Hutchinson's observations as to the peculiar notched chisel-like teeth, which he regards as characteristic of syphilis, for I have seen them in children quite free from any taint.

The result of hereditary syphilis in the majority of cases is fatal, especially in children born with some of its indications. In those in whom the symptoms become developed some time after birth it is not so fatal, but the prognosis is more unfavorable in proportion to the number of the products of the infection to the age of the child.

Rickets, scrofula, and fatty degeneration of various internal organs seem in many instances to be the indirect result of syphilis.

Etiology.-Numerous observations seem to establish the following interesting facts about hereditary syphilis. The great majority of the cases, probably nine tenths, are derived from the father. The male parent may procreate some children affected by hereditary syphilis, others affected by scrofula, and others who are entirely healthy; and the procreation of syphilitic children may take place without the mother suffering. When the mother is affected by secondary syphilis she rarely carries her child to the full time, and she may even abort seven or eight times in succession before she gives birth to a living child, and it will almost certainly bear some mark of the disease. If the mother become affected before a pregnancy, at the time of being impregnated, or during the early months of gestation, the child will almost certainly suffer; but if the infection does not occur till late in the period of gestation, the child will probably escape.

Children affected by syphilis may be nursed by the healthy mother without risk of her being infected, but wet nurses seem apt to catch the disease by the infection of their nipples from the sores on the child's lips. Nurses suffering from secondary symptoms do not seem to be able to infect by their milk children whom they are suckling, but they may do so if there is any ulceration about the nipple. Infection of the child during its 
birth by primary sores on the passages of the mother has not yet been proved.

Primary syphilis may be communicated to children by various accidents, among which is vaccination, and by attempted coitus. I have had under my care a girl eight years old infected with a primary sore by a boy aged ten.

Treatment.-When a mother is found to be subject to frequent abortions, the result of syphilis, or that children are born with the disease, the parents should be advised to undergo a course of treatment, especially the father.

'The method of nutrition of syphilitic children is always a point to which considerable attention should be directed. If the mother be able to suckle the child herself she should do so ; but if she cannot, some suitable substitute must be found; but it should not be placed at the breast of a healthy wet nurse until she has been made fully aware of the risk she may run. Günzburg denies entirely that hereditary syphilis can be communicated to nurses from children; but as $I$ have seen it so transmitted several times, I must take leave to dispute his assertion.

There is no drug which has so certain and so rapid an effect in the treatment of hereditary syphilis as mercury, though even that does not always conquer the tendency of the disease to return; and of all the mercurial preparations calomel seems to me to be the most serviceable, given in doses from a third to two thirds of a grain two or three times a day. In anæmic children it may be usefully combined with iron, and if there be any tendency to diarrhœa the addition of some Dover's powder will be of service. A very rapid and safe method of treatment is the mercurial inunction. The subcutaneous injection of the bichloride of mercury is a plan which has fallen into disuse from the tendency there is for the formation of obstinate ulcers at the seats of the injection. Mercurial vapour baths have been strongly recommended.

The preparations of iodine take a much longer time than mercury to affect syphilitic diseases, though sometimes in obstinate affections of the mucous membranes, bones, and glands, I have found it more useful than mercury given in the form of iodide of potash or iron. Sometimes great benefit is derived from a combination of the iodine and iron treatment. 


\section{NINTH DIVISION}

\section{DISEASFS OF THE SKIN}

Mestion need hardly be made here of the extreme importance to children of a careful attention to the condition and cleanliness of their skins, attention which must be the greater. the younger they are.

To keep children in good health they must be regularly bathed, not merely to clean their skins, but to keep them in proper action; and baths have an influence not merely on the skin, but on the subjacent muscles and blood-vessels, also on the nervous system and on the general tissue changes. In fact, the use of the bath should form as ordinary a part of the toilet as the use of the comb or the brush.

Warm baths are the best for cleansing, and cold baths are useful as a refreshing tonic, and as a means of hardening children against the evil influence of changes of temperature. The bath is also often a useful therapeutic agent, but certain precautions must always be regarded in its use, either in this direction or for more ordinary purposes, as to the temperature, the frequency of its use, and the time the child is allowed to remain in it; for error in any of these points may entail serious results.

The skin of the new-born child will be found covered with the vernix caseosus, and for the removal of this the best plan is first to rub on a little clean oil, butter, or lard, and then to wash the child with water of a temperature at about $38^{\circ}$, using some bran instead of soup. During the first nine months the infant should have a daily bath, a little above blood heat, and should not be kept in the bath more than six or eight minutes. After the seventh month the temperature of the bath may be 
slightly lower, but the child should not be kept so long in it. After the child is a year old a daily bath is not necessary, three or four times a week will be enough, with a temperature of $29^{\circ}$ or $30^{\circ}$; and for children over three years of age a bath once a week will answer every purpose. During a catarrhal attack affecting the chest the baths should be suspended, though a mere slight cough should not be sufficient to interfere with them. Care should always be taken not to allow children to play about unclothed before going into their bath; still more, after they. have come out. As a rule, children with skin eruptions should not be bathed often, and in water at as low a temperature as possible, for warm baths seem to increase the hyperæmia of the skin.

It is also a good plan to accustom children early to being washed, not bathed, in cold water, that is about $25^{\circ}$, especially about the anus and genitals, and when this plan is once begun it should be continued, even during feverish illnesses. When the child is so advanced that daily warm baths are no longer necessary, it is a very good plan to accustom them to have the whole hody washed daily with fresh cold water, as it has a powerful influence in hardening them. It ought to be done quickly, and may take place either in the morning or in the evening just before bedtime.

When the children have reached five or six years of age they ought to be accustomed to river- or sea-bathing, and be taught to swim; but care must always be taken not to allow them to remain too long in the water.

In certain diseased conditions medicated baths are advisable, and these may be divided into tonic, stimulant, and nutrient baths.

Of the first we may mention the malt bath, which consists of a decoction of malt, one to one and a half pints of malt being boiled and added to enough water to form a bath; the tan or oak-bark bath; and the Franzenbad bog iron salt bath, a pound of the salt being enough for a bath, which ought to be taken in the forenoon, at a temperature of $28^{\circ}$ or $29^{\circ}$, and for a period of six or eight minutes at first, and afterwards fifteen or twenty. The stimulant baths are constituted by the addition of aromatic herbs to the water, such as mint, hops, lavender, the needles of the pine, mustard, \&c.; and we include sea baths in this class. 
The nutrient baths consist of such mixtures as milk and water, equal parts, and beef-tea; and they were formerly much in vogue for weakly and atrophic children, though now they are not much used. The tonic and stimulant baths will often be found useful in the treatment of weakly, anæmic, rickety, and scrofulous children, and in those who are convalescent from acute asthenic diseases.

Cold douches may also here be mentioned, their use being either local or general. The cold douche is often applied to the head in affections of the brain; the water, at a temperature of $12^{\circ}$ or $14^{\circ}$, being allowed to run from a perforated vessel, held a short distance above the head for a few minutes.

\section{Erythema}

This affection occurs as a more or less diffuse hyperæmia of the skin, with inflammatory swelling of the papillary bodies, and is marked by redness, burning pain, and itching. It generally gets well rapidly, is of very common occurrence in infancy, and presents the following distinct forms :

Erythema neonatorum is a physiological reddening of the skin, which is seen after birth, especially strongly marked in some children, and which passes off into a yellow shade, and disappears in a few days.

Ery thema papulatum is a form also seen in new-born children, consisting of dark red prominent nodules of pin-point size, scattered over the skin, and surrounded by small rings of a fainter red colour. It is found on the face, trunk, and extremities, and especially on the backs of the hands and feet, giving rise to a sense of burning and itching, and it usually disappears in a few days, followed by slight desquamation. It is liable to be confounded with measles, but it may be distinguished by the absence of fever and the catarrhal symptoms which always accompany measles. It rarely requires treatment.

Erythema intertrigo is a variety due to some mechanical or chemical irritation, and is most frequently developed at those places where two folds of skin are in contact, especially at the genitals and the folds of the joints, being due to a superfluity of fat, tenderness of the skin, the access of urine, and a general 
inattention to the cleanliness of the child. If neglected it may result in abrasions or even serious ulcerations, which may take on a diphtheritic action or even become gangrenous, but it readily yields to a little care and the liberal use of puff poivder.

Erythema nodosum is most commonly found on the fronts of the legs, and sometimes on the arms and face, in the form of red or brownish-red spots, varying in size, hard and tender to the touch, and raised above the level of the surrounding skin. It is generally accompanied by some general symptoms of constitutional disturbance, with slight fever. It may continue for two or three weeks, coming out sometimes in successive crops, and always disappearing by resorption. It seems to have some association with the rheumatic diathesis, and is best treated locally by the application of some sedative lotion, such as one of acetate of lead, and the internal administration of a slightly laxative saline mixture.

Besides the erythemata above mentioned, there are others not of so much importance, such as e. solare, arising from exposure to great heat; e. traumaticum, from injury ; passive erythema, occurring in the course of some acute disease, as pneumonia; e. venenatum, due to the action of certain drugs and poisons, as iodine and belladonna; and the erythema seen in connection with pyæmia and diphtheria, which may be mistaken for scarlet fever. The administration of belladonna seems to have an especial aptitude for producing an erythematous rash very closely resembling the eruption of scarlet fever, but it is always accompanied by the peculiar indications of the physiological action of belladonna, such as wide dilatation of the pupil and great excitability, and therefore may be easily identified. This drug should always be given cautiously to children, and great care should be taken by the druggist in preparing prescriptions containing it.

\section{Erysipelas}

This disease is an inflammation of the skin, with cellular infiltration of the corium, and also of the subcutaneous tissue in severe cases. It is as often seen in new-born infants (erysipelas neonatorum) as in grown children. 
Symptoms and course.-We need only consider here the erysipelas neonatorum, for the disease in older children presents no difference to what is seen in adults. It generally makes its appearance first at the umbilicus, though sometimes elsewhere as on the penis after ritual circumcision, and is ushered in by febrile symptoms and redness of the skin, swelling and tenderness.

If the inflammation spreads the general symptoms increase in severity, the temperature rising, and the child suffering from great restlessness, nervous derangement, and even convulsions. Sometimes the disease spreads outwards whilst it leaves the skin first affected (e. migrans), or it may break out in other distant parts (e. ambulans).

Occasionally the inflammation takes on a phlegmonous character and ends in suppuration, this occurring most frequently on the sacrum and back of the hands and feet; and in the scrotum the inflammatory action may even become gangrenous, as has occurred twice in my practice.

Under all circumstances it is a disease of a dangerous character, some authors declaring that it is almost always fatal. It is very apt to be complicated by, or to lead to, such serious lesions as purulent peritonitis, meningitis, \&c., or general pyæmia. It seldom lasts more than a few days or a week, but sometimes it may be protracted for two or three weeks.

Causes.-Erysipelas generally has its origin in some local suppuration or injury, and it is specially apt to originate in this way in scrofulous children; but in some cases it seems to be the expression of a blood infection, perhaps of a pyæmic nature, and in others to be the result of some epidemic influence, especially in spring or autumn. The erysipelas of newly-born children almost always originates at the umbilicus or on the genitals.

Treatment.- No form of local treatment yet devised seems to have much effect in checking the spread of the disease, and nearly all we can do is to treat symptoms as they appear. Whilst the inflammatory symptoms predominate the best local applications are ice bladders or cold packing; or when this is objected to, carron oil which is equal parts of olive oil and lime water, may be applied on linen rags; and after the acute stage has passed, resorbents, such as iodine or mild mercurial ointment, should be used. When the febrile symptoms are severe, 
especially if they have a pyæmic character, quinine should be given.

\section{Dermatitis folliculosa (Acne cachecticorum)}

This is a disease almost confined to hospitals, and rarely scen in children attended in their own homes. It consists of an eruption of sinall nodules about the size of a hemp-seed, corresponding to the follicles of the skin and surrounded by a small inflammatory ring, of a very indolent character and which results in the formation of crater-like ulcers, which are always circular and have sharply-marked edges, and leave behind them excavated cicatrices. These ulcers may become confluent, and thereby reach a considerable size, or they may even form the point of origin of gangrenous destruction.

The more common seats of this eruption are the abdomen, buttocks, and back, and more rarely the inner surfaces of the thighs and legs. It never attacks healthy children, but only those who are cachectic or in a reduced state of health, especially those suffering from chronic intestinal catarrh, tuberculosis, and scrofulous diseases. It very often appears in successive crops, so that the ulcers of the first eruption may be only half healed before another crop comes out; and it is a very curious circumstance that the ulcers are almost always all healed before the patient dies, a result which is almost invariable in cases where they are formed.

This disease, besides being favoured by general cachexial conditions, may also be the result of continuous lying on the back and the absence of proper attention to cleanliness. Its pathology may perhaps be explained by thrombotic obstruction of the cutaneous capillaries, and the subsequent ulcerative destruction of the follicles.

For the treatment the most important consideration is a close attention to cleanliness, and the local application of some mild ointment, such as calamine cerate.

\section{Urticaria (nettle-rash)}

The eruption in this disease consists of wheals raised slightly 26 
above the level of the neighbouring skin, whitish in the centre and red at their periphery, due probably to an acute serous effusion into the superficial layer of the corium. They orcur all over the body, and are accompanied by intense itching and a burning pain.

In many cases urticaria is unaccompanied by any constitutional disturbance, whilst in others there is a good deal of fever and gastric derangement either before the appearance of the eruption or accompanying it. Sometimes the affection is limited to one attack, but in other cases it may recur at irregular intervals, and occasionally it may even become habitual. It is very often caused by irregularities of diet, or by the use of certain vegetables, fruit, or shell-fish, or by exposure to cold. It is also of very common occurrence in teething children, and sometimes occurs during the course of other diseases, such as smallpox, scarlet fever, measles, and cholera. The cases in which it becomes habitual are chiefly girls at the period of puberty. It may be regarded as a disease of the vaso-motor nervous system.

In cases where it is due to an error of diet it will generally yield to an emetic and a slight saline purgative, and in the habitual cases iron is the best remedy.

\section{Herpes}

This is an acute, non-contagious, vesicular eruption, the vesicles being generally of a uniform size, associated in groups, and having a reddish base. They are generally situated over the area supplied by a nerve twig (herpes genuinus), and are almost always accompanied by pain in that nerve, their appearance being sometimes preceded by shivering and fever. Very soon after the eruption has appeared the vesicies dry up, and the scabs drop off, usually without leaving any cicatrix ; but sometimes they ulcerate, especially in herpes zoster, and then they leave marks. The contents of the vesicles are alkaline, and sometimes are slightly mixed with blood.

The following varieties of herpes are met with in children :

Herpes facialis (hydroa febrilis), most common on the lips (h. labialis), but also seen on the cheeks, nose, forehead and ears, is a very frequent accompaniment of febrile diseases, 
especially pneumonia, intermittent fever, and the acute exanthemata, though it may also occur as an independent affection.

Bertholle has described a herpetic eruption of mucous membranes, especially of the gums, but I have not yet seen it in children.

Herpes zoster (shingles) is a disease which is almost always unilateral, though Hebra and others have seen it double-sided. I have scen one case where it was seated on both sides of the chest. The eruption is ushered in and accompanied by intense pain, which often, but by no means always, disappears with decrustation. In adults this pain sometimes continues months or years after the disappearance of the eruption, but this is not the case in children. According to the seat of the eruption the disease may be classified into $h$. zoster pectoralis, which is the most common variety, and begins usually over the spinous process of a vertebra, coursing along the track of an intercostal nerve until it reaches the middle of the sternum; h. z. abdominalis, corresponding to the course of the lumbar nerves; h. z. femoralis, which begins generally over the buttock, extends down the course of the sciatic nerve as far as the knee, or even to the calf of the leg, and has in some instances been followed by slight symptoms of paralysis; h. z. brachialis, a rare form, which begins in the neck over the last ccrvical vertebra, and extends down the arm; h. z. facialis, which follows the course of the first, or more rarely of the third, branch of the trigeminal nerve; h. z. capiliitii, which scems to affect the frontal and supra orbital nerves, and sometimes involves the conjunctiva. Herpes zoster may last from four to twelve days, and always gets well.

Causes.-Bohn has stated that herpes zoster is undoubtedly more common among children than among adults; and an opinion that it occurs only once in each patient is very general, but it has not been confirmed.

Boys seem to be attacked in a somewhat larger proportion than girls, and no agc scems exempt from it; though children between four and twelve seem most liable to it. Of all the patients admitted to our hospital, about one in 213 is a case of herpes zoster. Various pathological explanations have been offered of the discase, Thomas suggesting that it originates in an irritation of the corresponding spinal ganglia; other causes have also been 
mentioned, as peripheral inflammation, blows, unusual exertion, and blood poisoning.

The indications for treatment are, first of all, to prevent fractious children from scratching or rubbing the heads off the vesicles, and to mitigate the pain, for which latter purposes the local application of cold, or the administration of opiates either internally or by subcutaneous injection in the neighbourhood of the eruption will be found the best.

Herpes iris is a form in which a central vesicle is encircled by a group of others, so that the most recent are always farthest from the centre of the group, the name iris being due to the various shades of colour thus produced br the various stages of efflorescence in the group. It occurs most frequently on the backs of the hands and feet, more rarely on the other parts of the limbs, and very exceptionally on the trunk and face. It usually lasts about eight or twelve days, and goes through the same involution as the other varieties of herpetic eruption. It rarely requires any treatment, but if persistent a mild ointment of the red oxide of mercury is the best application.

\section{Pemphigus and pompholyx}

This eruption consists of round or oral bullæ of various sizes, filled at first with clear serum, which afterwards becomes turbid from the presence of pus, these bullæ occurring over any part of the whole surface, and the rest of the skin remaining in its normal condition. After a time they burst, and their sites become covered with thin crusts, which, when they fall off, display slightly reddened surfaces which slowly resume the normal appearance of the skin. In one case, however, I observed that they left white scars. Sometimes the contents of the bullæ are mixed with blood, and in some rare cases they contain nothing but blood-pemphigus hæmorrhagicus. When serous. the fluid is generally neutral, becoming alkaline in its progress ; but in a few cases I found it acid; and Bamberger has found in $t$ fibrinous threads, leucine, tyrosin, ammonia, but no uric acid.

Very often the disease affects the whole skin, including the hairy scalp. but sometimes it is localised to some particular. 
region, the most common being the lower extremities, trunk. genitals, face and neck, and more rarely the scalp, palms of the hands, and soles of the feet, the latter being; on the contrary, almost always the seat of syphilitic pemphigus. Occasionally we see the bullæ of pemphigus on the mucous membrane of the mouth and nose.

The disease may be met with in either the acute or chronic form, the former, or idiopathic pemphigus, being almost confined to children, and in them is not unfrequertly accompanied by severe constitutional disturbance of a febrile character, and may last from three to six weeks.

The chronic pemphigus, which occurs in children as p. vulgaris, differs from the acute pemphigus in that the eruption comes out anew at intervals of a few days, weeks, or months, or as soon as the first bullæ have disappeared others appear close to them, and spread in a serpiginous manner, the p. serpiginosus of Hebra, the appearance of the bullæe being preceded by an itching and burning of the skin, and the whole course of the disease being free from fever.

The acute disease is not often complicated, but the chronic form is sometimes associated with intestinal catarrh and bronchitis, and often ends in marasmus. I have also seen hæmaturia as a rare complication, and in one fatal case there was albuminous néphritis.

Pemphigoid eruptions are much more common in children than in adults, and girls seem more frequently subject to it than boys. In my own experience the great majority of the cases occurred in children under one month old, and the month of July seems specially favorable for its production. In my hospital cases, one in 700 has been a case of pemphigus. Its production seems to be favoured by a weakly and reduced condition of the child, and an alnost special form may be described - p. cachecticus 'v. pyamicus - as occurring in children with suppurative diseases and pyæmia.

Acute pemphigus also occurs in healthy well-nourished children, and the syphilitic variety is met with in the unfortunates suffering from the hereditary disease. Pemphigus does not seem to be either contagious or innoculable, but cases occur in batches every now and then.

The acute disease nearly always gets well, but the chronic 
form is very obstinate, and with its complications is sometimes fatal, and often apparently incurable. Of seventy cases sixteen have bcen fatal, ten of them suffering from syphilitic pemphigus, four from the cachectic disease, and two from the pyrmic form.

Treatment.-Acute pemphigus requires nothing but some slight febrifuge and the local application of some puff powder or zinc ointment. In the chronic disease, if there be the appearance of any cachexia it must be treated constitutionally, as indicated elsewhere; but little results will be obtained from any local applications.

\section{Eczema}

Eczema is an inflammation of the skin, sometimes acute but more commonly chronic, in which small papules, vesicles, or pustules are formed, followed by the formation of crusts, and accompanied by intense itching. It may bc divided into several varieties, according to the anatomical relations of the inflammatory product, such as e. simplex v. vesiculosum, when the epidermis is raised in the form of small vesicles; e. papulatum, if only simple papules are to be seen; e. impetiginodes, when the vesicles are of large size and their contents turbid or purulent, and when they form yellow or brownish crusts after bursting; e. squamosum, when the inflamed spots are red and scurfy; e. rubrum, when the skin becomes intensely red, and exudes moisture. If the exudation affects also the deeper tissues of the skin, the eczema becomes complicated with œedematous swelling of the skin; and in chronic eczema this is often the case, the skin becoming thickened, rough, and fissured, with papillary hypertrophy and cellular infiltration of the deepest layers of the corium.

Eczema may affect the whole surface of the skin (e. universale), or it may be limited to some particular part (e. partiale). Its favourite sites in children are the scalp (e. capillitii, tinea capitis achorosa), the face (e. faciei, crusta lactea), the lines of union of mucous membrane and skin, the extremities, and especially at the folds of the larger joints and the neighbourhood of the arms and genitals.

In acute eczema the eruption is generally accompanied by 
febrile symptoms, and in every case where the eruption is very extensive the lymphatic glands are apt to enlarge and suppurate, and such complications as furuncular inflammation of the skin, erysipelas, urticaria, general anæmia and wasting, or even parenchymatous nephritis may be met with.

Eczema is frequently seen as a complication of other papulous and pustulous diseases of the skin, as prurigo, ecthyma, lichen, \&c., especially in scrofulous children.

This skin disease may be due to some external cause or to some circumstances which arise in the system of the child. Of the former class may be mentioned exposure to extreme degrees of temperature, mechanical or chemical irritation, such as rubbing or pressure on the skin by some badly adjusted article of dress, the presence of animal parasites. Of the latter class the most important is the scrofulous taint, for out of 1192 children with scrofulous diseases, 684 had affections of the skin, and, for the most part, they were eczematous. Dyspepsia, rickets, and teething, are also frequent causes of this disease; and acute diseases, such as measles, hooping-cough, scarlet ferer, typhoid, \&c., often bring back an eczematous eruption which has disappeared, or intensify one already present.

Eczema is not contagious, but it spreads often from one piece of skin to another by prolonged contact. In very many cases it is a mild and easily curable affection, but in others it is very obstinate or even incurable, and it is very subject to relapses, especially when it has a scrofulous origin.

It seems more difficult to remove from some parts than from others, as, for instance, from the scalp, hands, and eyelids.

Treatment.-The question whether chronic eczema in children, especially of the head and face, should be locally treated at all, is one on which considerable difference of opinion exists, some authors asserting that it should, whilst others, such as Waldenberg, recommend as strongly that.it should be left entirely alone as far as any local measures are concerned, lest the curing of the rash induce mischief of a more serious kind, as meningitis, hydrocephalus, \&c. More than a thousand cases of eczema have come under my own observation, and alınost all have been subjected to local treatment without respect to their duration or extent, and I do not know that any fatal case has occurred during or after such treatment. On the contrary, I have had 
repeated experience that the cure of the eczema has been followed by relief of serious diseases which have been caused by the distress and restlessness due to the eruption. I am therefore in favour of local treatment, combined with the constitutional treatment necessary for any dyscrasia which may be detected.

In the local treatment of eczema I have found cold packing or frequently repeated cold douches very useful, or the use of fomentations with a lotion of acetate of lead, as long as there is any evidence of hyperæmia of the skin. During the vesicular stage ointments of oxide or acetate of zinc, alum, white bismuth, or white precipitate, will be found very efficacious; though, perhaps, the best of all applications is the unguentum dianchyli albi of Hebra, which consists of equal parts of linseed oil and diachylon plaster, to be applied on rags and changed every twelve hours. In squamous eczema the use of pitch or oil of cade ointments, or a weak carbolic-acid lotion, may be tried; and in the variety which is found in flexures of skin, some kind of puff powder, as simple starch, oxide of zinc, \&c., will almost always be sufficient.

\section{Lichen}

Lichenous eruptions are characterised by small pin-point nodules, somewhat redder in colour than the normal skin, or of a dirty brown colour, not accompanied by much itching, and followed by slight furfuraceous desquamation. Hebra has described two varieties: the lichen scrofulosorum, and the lichen exudativus ruber. Some authors still regard strophulus as a separate disease, but it cannot be regarded truly as other than a lichen, for it differs from this class of eruptions in nothing except that it occurs in sucklings and teething children, and it seems to me a pity to keep up the separation.

L. scrofulosorum is the variety which most affects children, its usual seat being on the trunk, though it may also be met with on the extremities, and even on the face. As the name implies, it is seen only in scrofulous or tuberculous children, is often associated with other scrofulous diseases of the skin, mucous membranes, and bones. Like all such diseases, it is extremely obstinate and apt to return, and is seen mostly in children somewhat grown, but sometimes in those under two years of age. The pathological change in the skin seems to be the 
removal of exudation cells from the epidermic and adipose follicles.

The treatment should be both local and constitutional. Hebra recommended inunction with cod-liver oil, and that the child should be clothed in woollen materials. I have used Vlemingkx' solution, white or red precipitate, or tar ointment, or Hebra's diachylon ointment with benefit.

Lichen exudativus ruber is much less common in children, and it differs in no way from the disease when seen in adults.

\section{Prurigo}

The eruption in this disease consists of small papules of a light red colour, always accompanied by an intense itching, so that they get scratched and are generally seen with their heads torn off and covered by brownish-red crusts. If the eruption has become chronic the skin is thickened and pigmented, rough, hard, and fissured, and the superficial lymphatic glands, especially in the groin, become enlarged. It is generally limited to the flexor aspects of the limbs, more commonly the lower, and is met with on the trunk only exceptionally, and the violent scratchings, which the patients always indulge in, give rise to many sores, pustules, \&c.

Children who suffer from this skin disease are almost always those living in poor circumstances, and generally about five years of age, though sometimes they are younger, and from the loss of sleep which it always involves they are generally much reduced in health.

It seems to be due to an inflammation of the papillary bodies, but the further cause is yet unknown. It does not seem to have much connection with scrofula. It is always chronic, obstinate, and very apt to return, and if neglected, may even become quite incurable.

With children who have delicate skins it is advisable to begin the treatment with very mild remedies, such as inunction with lard, weak tar ointment, or cod-liver oil. For older children and more pronounced forms of the disease I have found Vlemingkx' solution the best remedy, followed after ten or

1 This is made by boiling equal parts of sulphur and quicklime in six times the bulk of water until half the water is evaporated, then filter when coll. 
twelve applications by the use of tar ointment, or an ointment of white precipitate and Peruvian balsam. Cold packing will be found the most efficacious means of relieving the intense itching, and warm baths should not be used except for purposes of cleanliness. The internal administration of cod-liver oil, iron, arsenic, and the preparations of iodide of potassium, may all be tried in obstinate cases. Care should be taken to prevent the child scratching as much as possible by frequently cutting the finger-nails, muffling the hands, and protecting the affected parts.

\section{Seborrhoea}

Under this term is included any condition by which the secretion of the sebaceous glands is abnormally increased, a condition which is chicfly found in children on the scalp (seborrhœa capillitii). It is of most frequent occurrence in children under a year old, especially in those whose cleanliness is not very well looked after, and sometimes the secretion, mixed with epithelial detritus, dirt and dust, may be seen agglutinating the hair into a hood-like covering, its favourite seat being the front of the scalp, especially at the great fontanelle. The growing hair gradually lifts this up, and there is seen underneath it the normal young epidermis, somewhat reddened. This condition of the scalp is seen quite as often in well-nourished and healthy children as in those which are weakly and diseased. It is sometimes met with as a complication of eczema.

For the removal of the crust it should be softened with some oil or greasy application, and then washed off, and some mild astringent ointment applied to the skin.

\section{Alopecia areata, Area Celsi, Porrigo decalvans (Willan)}

In this disease the hair falls off in circular or irregularlyshaped patches, leaving the skin perfectly bald, shining, and destitute of pigment. The bald patches grow at their circumference, and the nearest hair comes out with very slight pulling. The disease may limit itself to certain patches, or it may extend till the whole body is entirely destitute of hair.

It is regarded by some authors (Gruby and others) as a para. sitic disease, and by others as a trophoneurosis, whilst Rind- 
fleisch regards it as due to a defect in the nutrition of the hair, whereby the root undergoes fatty degeneration between the middle and lower third, and by that process the hair becomes amputated. From my own observations I cannot hold it to be parasitic, and I have failed to notice its innoculability men. tioned by Ziemssen.

The majority of cases occur in delicate children between four and ten years of age, though I have also seen it in very healthy and well-nourished children. A cure can always be obtained, though the disease is liable to return.

The treatment should consist in the internal administration of quinine and iron, and the local application of stimulant lotions and ointments, such as white precipitate ointment, or Rindfleisch's mixture of glycerine and tincture of capsicum.

\section{Sclerema}

This peculiar condition of the skin is found almost solely in newly-born children. It is marked by induration and oedematous swelling of the skin and subcutaneous cellular tissue, and a very remarkable lowering of the temperature.

The disease is generally seen first in the skin of the calf within three or four days after birth, in the form of circumscribed, insular spots of hardness, or it may be more diffused and extend gradually up the thighs to the trunk, upper extremities and face; the latter region, or indeed any other part of the skin, may occasionally afford its point of origin.

The affected skin becomes blanched, waxy, and glistening, or it may put on a yellowish tinge, and feels hard and cold to the touch. The limbs become thick, misshapen, stiff and immovable from the odema, so that altogether the body looks as if it had been frozen. By this time, or perhaps somewhat earlier, the child becomes weak and somnolent, refuses the breast, the breathing becomes rapid and superficial, the voice weak and whimpering, the pulse small and retarded, and the temperature falls eight or ten degrees below its nornal ratio. Towards the end of the case a discharge of bloody serum often cccurs from the mouth and nose. Very few cases recover, and on post-mortem examination we find a collection of yellow or 
reddish coloured serum in the skin and subcutaneous cellular tissue, very often pulmonary atelectasis, catarrhal or croupous pneumonia, hæmorrhagic infarctus of the lungs, capillary extravasations in various organs, serous effusions into the pleura and peritoneum, and sometimes permanency of the peculiarities of the foetal circulation, this being probably a mere coincidenee.

Hervieux and Löschner have described, under the term Algidité progressive, a condition which is somewhat similar, but neither so well marked nor so extensive. It consists of œdematous swelling, coldness and blueness of the feet, legs, and sometimes the thighs, which is met with in children suffering from chronic intestinal and pulmonary diseases a few days before death. Hæmorrhagic effusions into the skin are also occasionally associated with this condition.

The sclerema of children cannot in any way be regarded as a local disease, but is in all probability the result of central lesions of the respiratory and circulatory systems, as evidenced by the insufficient breathing and the diminished activity of the circulation, the whole depending probably on an insufficient innervation. It occurs more frequently amongst the children of the poor than anongst those of the well-to-do, more frequently in winter than in summer, in hospitals, maternities, \&c., than outside, and chiefly in delicate marastic children.

The treatment must be directed to the improvement of the general condition of the child, the administration of strong nutritious diet, and to keeping up the temperature and the force of the circulation, by wrapping the child in cotton wool, and the administration of stimulants.

\section{Parasitic diseases of the skin}

There are two classes of parasites which infest the skin: the vegetable and the animal; the former producing such diseases as herpes tonsurans, and favus, and the latter giving rise to scabies.

Herpes tonsurans, Herpes circinatus (Bateman), Trichomykosis, ringworm, Trichophyton tonsurans

This disease is due to the presence of a parasite, the tricho- 
phyton Malmstenii, and occurs chiefly in the hairy scalp, though it is also met with on parts of the skin destitute of hair. It begins as round spots, somewhat raised, of several lines in diameter, with small whitish scales in the centre, and the peripheral zone somewhat red (herpes tonsurans maculosus) : or it may occur in the form of minute, though quite visible vesicles, circularly arranged, which soon dry up and leave small thin scales behind them. If the disease attack the hairy scalp the hairs pull out or break off a little above the surface, so that circular patches of baldness are produced, and in this position the disease is very chronic, but when it does get better the hair comes back gradually.

This skin affection is much more frequent in children than in adults, and it is one which is very contagious and apt to spread by common use of combs, brushes, \&c. It is also communicated from the lower animals to children, especially from dogs, cats, horses, and cows. It may also be communicated from favus crust, for the investigations of Hebra, Neumann, Pick, and others have established that both favus and herpes tonsurans may be produced by one parasite (penicillium).

It sometimes gets well spontaneously; but careful washing with alkaline soap will very much expedite its disappearance; and if it resist such simple treatment, a solution of borax or an ointment of red precipitate will seldom fail to cure it.

\section{Favus, tinea vera, porrigo favosa}

This disease may be known by its peculiar white or sulphuryellow crusts, either isolated or collected in groups, cup-shaped, and very much resembling honeycomb, with a peculiar mousey smell. Its favourite seat is the hairy scalp, though it may also be found in other parts of the skin. At its commencement the skin becones marked with slight hyperæmic spots, round or diffused, which itch very much, and which gradually rise into little yellow eminences, become pustular, and form the peculiar crust. According to Köbner it generally begins in the form of herpetic vesicles. If the scab be removed there will be found beneath it a small cup-shaped indentation with a delicate reddencd epithelial covering (partial atrophy of the skin), or if the disease has been of long duration there may be actual ulceration, 
and the affected hairs are dull and colourless, and covered with a fine powder, and very brittle. Microscopic examination of the crust, epidermic cells or hairs and hair bulbs, reveals the presence of a parasite, the achorion Schönleinii, composed of numerous colourless mycelium fibres and spores, arranged in meshes.

Whether favus and herpes tonsurans are due to one and the same parasite in different stages, as stated by Robin, Hebra, Hutchinson, Pick, and others, or whether the parasites are different altogether, as believed by others, is a question yet open, though the balance of evidence is in favour of the former view; but further investigations are needed.

Favus attacks children as well as adults, and is communicable from one patient to another, and from the lower arimals to man. It is a very chronic and obstinate disease, and sometimes seems absolutely incurable.

In treating this disease the crust should first be softened by oil or some greasy application, and then carefully washed off; then the diseased hairs should be pulled out, and some parasitical remedy applied. For this latter purpose Hebra advises the skin to be rubbed twice daily with Dutch soap, and covered with compresses soaked in a solution of 1.5 per cent. of carbolic acid. Lotions of corrosive sublimate, benzin, and turpentine have also been recommended, and I have found considerable benefit from the use of styrax in several cases.

\section{Scabies (itch)}

This disease is due to the presence of an animal parasite-the acarus scabiei or sarcoptes-which is from a fourth to a fifth of a line in length, and about a sixth or seventh in breadth, and shaped not unlike a turtle, the male being considerably less than the female. The latter penetrate by means of their jaws through the epidermis as far as the Malpighian layer, and there deposit their eggs, their track through the skin forming a little straight or zigzag line, which is very characteristic of their presence, and by following which carefully with a needle they may often be caught.

The favourite positions for their ravages are between the fingers, at the elbows, axilla, penis, buttocks, soles of the feet; 
but sometimes they attack the skin elsewhere, even on the face and scalp. They give rise to intense itching, and the scratching which results produces sores, pustules, ecthymatous and even pemphigoid eruptions, so that sometimes when the skin is very tender and the disease has been a long time in existence; the poor child presents a most pitiable appearance. The itching always gets worst in bed after the patient has got warm. When the disease is recent its diagnosis is an easy matter, but when to it are added the complications mentioned above, it is no easy matter sometimes to identify it. Finding the acarus is the sign absolute.

In the early stage styrax and Peruvian balsam may be applied mixed with clive oil or glycerine. Sulphur ointment is a very good application, but the best is Vlemingkx' solution, either undiluted or diluted, if there be great irritation of the skin, and the eruption be very extensive. Monti recommends balsam of copaiva or a lotion of carbolic acid as the best local applications. 



\section{A P PENDIX}

\section{RULES FOR THE MANAGEMENT OF INFANTS}

\section{Warmth, Cleanliness, Fresh Air}

Ieep them warm: Net the clothing be warm, but not tight. Wash them all over with warm water daily, wiping them thoroughly dry afterwards. Never let a wet napkin remain on for a minute. Give them plenty of fresh air: send them out, at least for a short time, every day that the weather is fine; and, while they are out, air the room by freely opening the window.

\section{Nourishment while the Child is under Seven Months old}

The mother's milk is the proper food for infants. Therefore, if the mother has plenty of milk, let her suckle her child, and give it nothing else, till it is seven months old. If the mother has too little milk, still let the child have what there is; and, in addition, cow's milk and water, as directed in Rule 3. Till the child is seven months old, milk of some sort must be its only food. It is better that a mother should not work from home, for an infant requires its meals regularly, and needs many attentions which only a mother can give; hard work also makes the milk unwholesome.

\section{How to Bring Up "by Hand."}

If the child must be brought up by hand, it should be fed with warm sweetened milk and water out of a bottle. If the milk be genuine, add to it, at first, nearly half water; at a month old, add only one quarter part water. In most town milk, two table-spoonfuls of boiling water to the pint will be 
enough ; add also one table-spoonful of lime water (unless otlerwise advised), and sweetcn with white sugar. A child a month old should have about two pints a day of milk thus prepared, and not more than half a teacupful at a time; gradually put less water, and at four or five months give the milk plain. Give the child no other nourishment whatever, except under special advice. A very large number of the children that are brought up by hand die in childhood; and this mortality is for the most part due to the practice of beginning too soon with gruel, corn-flour, bread, arrowroot, \&c. These are not proper nourishment for children under seven months old, and should never be given to them. The bottle should draw easily. It should be very carefully washed out after every time it is used. Then bottle, cork, and tube should be kept separately in a bowl of clean water (containing a pinch of.soda) till the next time they are needed. If the bottle is not quite clean, the milk will sour, and will thus make the child ill. The "condensed milk" is good, and may be used if other milk cannot be got fresh.

To make Lime Water.-Put a piece of quick lime, weighing about half a pound, into a stone jar, or any earthen vessel, and pour gently and gradually upon it about a gallon of cold water, then well shake or stir it, and let it stand through the night, when the lime will sink down to the bottom. Next morning take off the top scum and pour the clear liquor into clean bottles, and well cork them.

\section{Importance of Regular Feeding}

The child should be put to the breast regularly-for the first six weeks, during the day, in general not oftener than every two hours; afterwards, about every three hours. During the night it does not need to be fed so often. A child soon learns regular habits as to feeding. It is a very great mistake to give the breast to the child whenever it cries, or to let it be always sucking, particularly at night: this is bad for both mother and child. If the child is brought up by hand, it should be fed with the same regularity; never give it the bottle merely to keep it quiet. If the child is weakly, the time between the feedings must be rather less, both during the day and during 


\section{Nourishment when the Child is over Seven Months old}

If at seven months the child is strong and healthy, and has cut a few teeth, it may now have one or two meals a day $\mathrm{cf}$ milk slightly thickened with good well-baked bread, Liebig's infant food, or Dr. Ridge's patent cooked food, or Chapman's entire wheat flour, or rusks, or well-boiled oatmeal, \&c. It should still have, besides this, plenty of plain breast or cow's milk. At ten months it may, once a day, have a little meatbroth made with barley or rice, without vegetables. At from ten to twelve months it should be taken altogether from the breast. Till the child is nearly two years old, no solid animal food should be given. 'Even at two years, milk should still be the chief food. Any meat should be well pounded.

\section{Avoidance of Stimulants, \&c.}

Tea, beer, brandy, and other'stimulants, cheese, new bread, fruit, and pastry, as also "soothing-medicines," "sleepingdraughts," " coordials," " teething-powders," \&c., should never be given; and even ordinary medicines should, if possible, be given only after proper medical examination and advice.

Children's Hospital (OUt-patient Departanent), Steelhodse LaNe, BirminghaM. 



\section{INDEX}

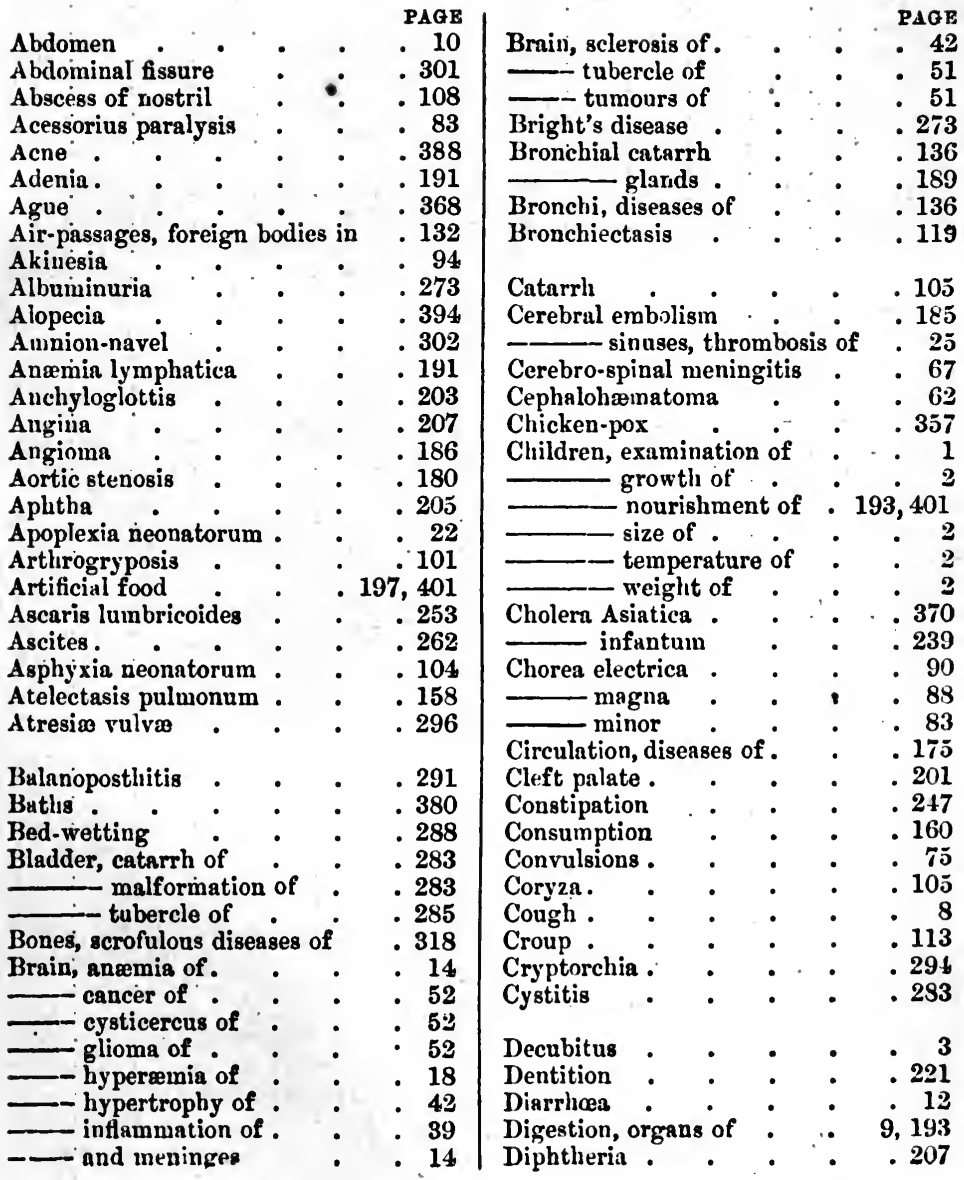




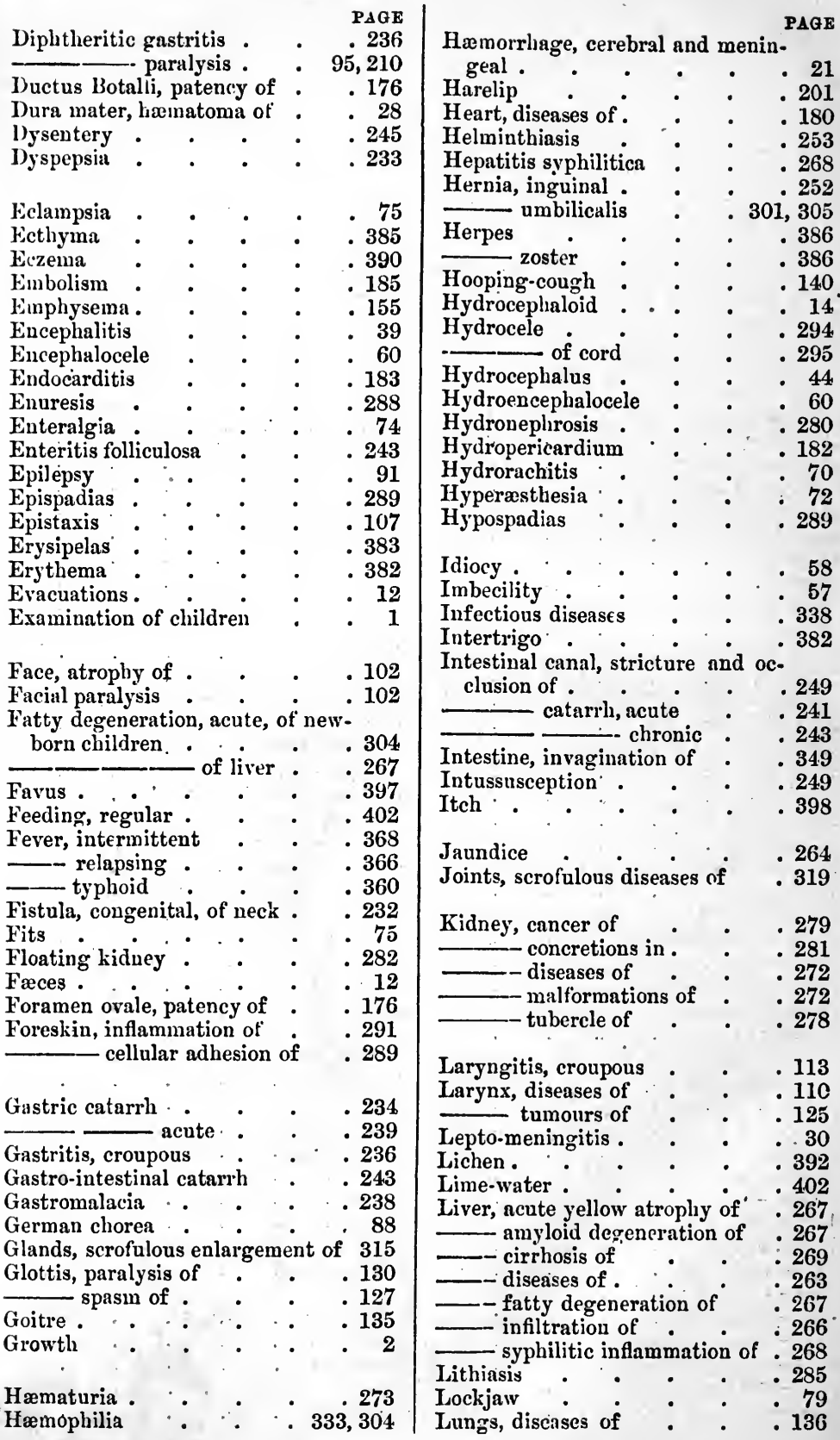




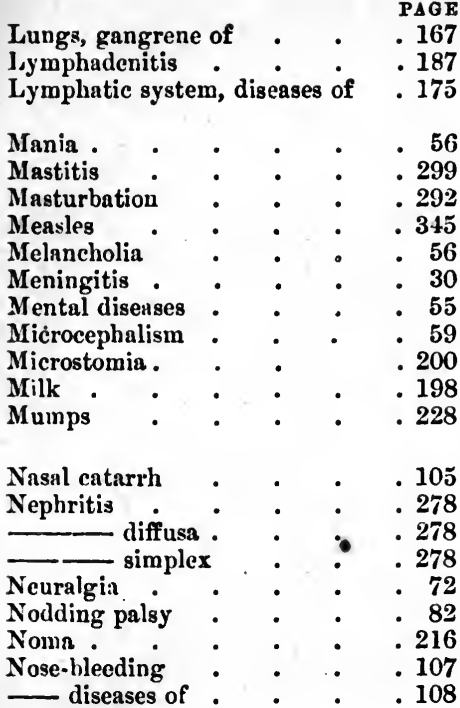

Esophagus, diseases of $\quad . \quad \therefore 230$

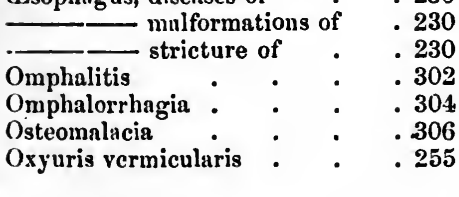

Pachymeningitis - • . $\quad 28$

Paralysis . . . . . 97

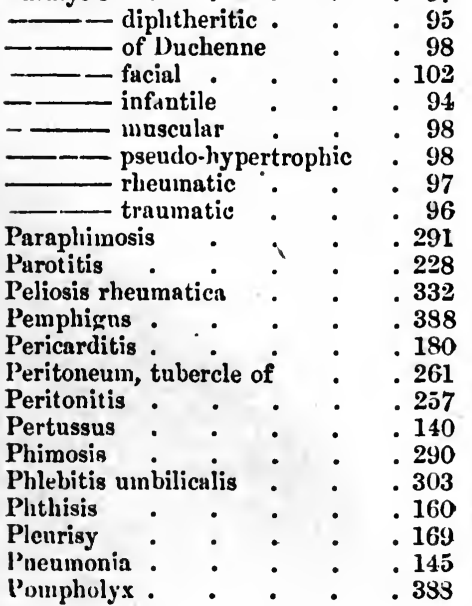

PAGE

Prepuce, adhesion of . $\quad . \quad 289$

Prolapsus ani . . . . 250

Prurigo $\quad$. $\quad$ • $\quad$ • $\quad$. 393

Pseudo-croup . . . $\quad .110$

- hypertrophic paralysis . 98

- leucocythæmia . . 191

Pulınonary artery, stenosis of .178

Pulse . . . . . . . 191

Purpura . . . . . 331

Rachitis . . . . . 306

Ranula . . . . . 203

Rectum, polypus of . . . 251

Retro- csophageal abscess $\quad . \quad 232$

- pharyngeal abscess $\quad . \quad .227$

Rickets . . . . . 306

Ringworm . . . . $\quad 396$

Rheumatism . $\quad . \quad .334$

Rötheln . . . . . 349

St. Vitus's dance . $\quad$. $\quad$. $\quad$. 83

Salaam convulsion $\quad \cdot \quad \cdot \quad 82$

Sarcoinphalus . . . $\quad .302$

Scarlet fever $\quad$. . . 338

Scabies . . . . . 398

Sclerema neonatorum : . $\quad .395$

Scrofula $\quad . \quad . \quad . \quad . \quad .313$

Seborrhœa . $\quad . \quad$. $\quad .394$

Septicæmia diphtheritica $\quad . \quad 210$

Skin, diseases of . $\quad . \quad .380$

- scrofulous diseases of . $\quad .316$

Simallpox . . . . . 350

Spasulus nutans . $\quad$ • $\quad$ • $\quad$ - 82

Spina bifidn . . . . 70

Spinal cord, anæuia of $\quad . \quad 64$

— diseases of $\quad . \quad 64$ hyperæmin of . . 64 tumours of $\quad . \quad 69$

- meningitis . : . 65

- palsy $\quad \cdot \quad \cdot \quad \cdot \quad \cdot 94$

Spleen, diseases of $\quad . \quad . \quad 270$

Splenitis . . . . . . 271

Stomach, diseases of . . . $\quad .233$

- follicular ulceration of . 233 perforating ulcer of $\quad .237$ self-digestion of . . 238

- tnbercular ulceration of .238

Stomatitis . . . . . 204

—_ crouposa $\quad . \quad .207$

— ulcerosa $\quad . \quad .215$

Stomatomykosis $\quad$. $\quad$. $\quad 219$

Stone in the bladder . . . 285

Syphilis . . . . . . 375

- $\quad$ of spleen $\quad . \quad 27$ !

Syphiloma of brain . . $\quad .52$

Tables mescraica . $\quad$ • . 243

Truia . • • • . . 256

lapeworm : : : : $: 256$ 


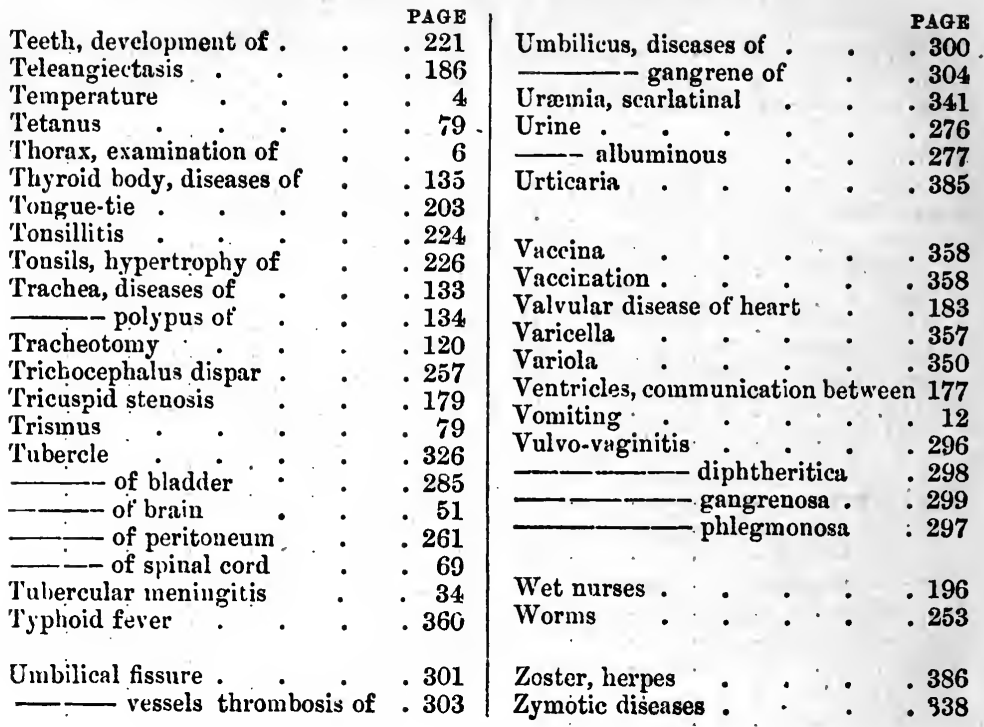




\section{AMERICAN CYCLOPEDIA.}

\section{NEW REVISED EDITION.}

Entirely rewritten by the ablest writers on every subject. Printed from new type, and illustrated with Several Thousand Engravings and Maps.

THE work originally published under the title of THE NEw AMERICAN CrCLopediA was completed in 1863 , since which time the wide circulation which it has attained in all parts of the United States, and the signal developments which have taken place in every branch of science, literature, and art, have induced the editors and publishers to submit it to an exact and thorough revision, and to issue a new edition entitled THE AMERICAN CYCLOPADIA.

Within the last ten years the progress of discovery in every department of knowledge has made a new work of reference an imperative want.

The movement of political affairs has kept pace with the discoveries of science, and their fruitful application to the industrial and useful arts and the convenience and refinement of social life. Great wars and consequent revolutions have occurred, involving national changes of peculiar moment. The civil war of own country, which was at its height when the last volume of the old work appeared, has happily been ended, and a new course of commercial and industrial activity has been commenced.

Large accessions to our geographical knowledge have been made by the indefatigable explorers of Africa.

The great political revolutions of the last decade, with the natural result of the lapse of time, have brought into public view a multitude of new men, whose names are in every one's mouth, and of whose lives every one is curious to know the particulars. Great battles have been fought and important sieges maintained, of which the details are as yet preserved only in the newspapers or in the transient publications of the day, but which ought now to take their place in permanent and authentic history.

In preparing the present edition for the press, it has accordingly been the aim of the editors to bring down the information to the latest possible dates, and to furnish an accurate account of the most recent discoveries in science, of every fresh production in literature, and of the newest inventions in the practical arts, as well as to give a succinct and original record of the progress of political and historical events.

The work has been begun after long and careful preliminary labor, and with the most ample resources for carrying it on to a successful termination.

None of the original stereotype plates have been used, but every page has been printed on new type, forming in fact a new Cyclopredia, with the same plan and compass as its predecessor, but with a far greater pecuniary expenditure, and with such improvements in its composition as have been suggested by longer experience and enlarged knowledge.

The illustrations, which are introduced for the first time in the present edition, have been added not for the sake of pictorial effect, but to give greater lucidity and force to the explanations in the text. They embrace all branches of science and of natural history, and depict the most famous and remarkable features of scenery, architecture, and art, as well as the various processes of mechanics and manufactures. Although intended for instruction rather than embellishment, no pains have been spared to insure their artistic excellence; the cost of their execution is enormous, and it is believed they will find a welcome reception as an admirable feature of the Cyclopxdia, and worthy of its high character.

This work is sold to subscribers only, payable on delivery of each volume. It will be completed in sixteen large octavo volumes, each containing about 800 pages, fully illustrated with several thousand Wood Engravings, and with numerous colored Lithographic Maps.

\section{Price and style of Binding.}

In extra Cloth, per vol. . . . \$5.00|In half russia, extra gilt, per rol. 8.00 In library leather, per vol. . . G.0O In full mor. ant.gt. edges, per rol. 10.00 In half turkey morocco, per vol. . 7.00 In full russia per rol. . . . . 10.00

Five volumes now ready. Succeeding volumes, until completion, will be issued once in two months.

* * Specimen pages of the AMerican Cycloredia, showing type, illustrations, etc., will be sent gratis, on application.

FIRST-CLASS CANVASSING AGENTS WANTED. 


\section{THE POPULAR SCIENCE MONTHLY,}

(Established Mray, 1872.)

\section{Conducted. by Prof. E. L. TOUJAANS.}

The Porular Science Montrily was started to promote the diffusion of valuable sci. entific knowledge, in a readable and attractive form, among all classes of the community, and has thus far met a want supplied by no other periodical in the United States.

The great feature of the magazine is, that its contents are not what science was ten or more years since, but what it is to-day, fresh from the study, the laboratory, and the experiment: clothed in the language of the authors, inventors, and scientists themselves, which comprise the leading minds of England, France, Germany, and the United States. Among popular articles, covering the whole range of Natural Science, we have the latest thoughts and words of IIerbert Spencer, and Professurs Iluxley, Tyndall, and R. A. Proctor. 'Since the start, it has proved a gratifying success to every friend of scientific progress and universal education; and those who believed that science could not be inide any thin: but dry study, are disappointed.

The press all over the land is warmly commending it. We subjoin a few encomiums from those recently given :

"That there is a place for Tire Popelar Science Moviule, no one can doubt wlio has watched the steady increase of interest in scientific inves tigation manifested in this country, not only by a select class, but by the entire community." - New York Times.

"A journal which promises to be of eminent value to the cause of popular education in this country."-New York Tribune.

It is, beyond comparison, the best attempt at journalism of the kind ever made in this country." -Home Journal.

"The initial number is admirably constituted." -Evening Mail.

"We think it is not too much to say that this is the best first number of any inagazine ever published in America."-New York World.

"It is just what is wanted by the curious and progressive mind of this country, and ought to bo widcly circulated."-New York Evening Post.

"It is the first successful attempt in this country to popularize science in the pages of a monthly." $-N$. Y. School Journal.

"Not the less entertaining because it is instructive."-Philadelphia Age.

"The Mosthly has more than fulflled all the promises which the publishers made in the prospectus of publication."-Niagara Fralls Gazette.

"It places before American readers what the ablest men of science throughont the world write about their meditations, speculations, and discoverlos."-Providence Journal.

"This is a highly-auspleions beginning of a uso. ful and much-needed enterprise in the way of pub. lication, for which the public owe a special debt of obligation to Messrs. D. Appleton \& Co."-Boston Gazette.

"This new enterprise appeals to all who are interested in the laudable effort of diffusing that in formation which is best calculated to expand the mind and improve the conditions, and enhance the worth of life."-Golden Age.

"Just the publication needed at the present day." Montreal Gazette.

"This new inagazine, in onr estimation, has more merit than the whole brood which have preculed it."-Oswego Press.

In our opinion, the right idea has been happily hit in the plan of this new monthly."-Buffalo Courier.

"This is one of the very best periodicals of its kind pubiished in the world. Its corps of contributors comprise many of the ablest minds known to science and literature. It is doing a great and noble work in popularizing science, promoting the growth of reason, and leveling the battlements of old superstitions reared in the childhood of our race before it was capable of reasoning." - The American Melical Journal, St. Louis, Mo.

"This magazine is worth its weight in gold, for its service in educating the people."-The American Journal of Education, St. Lous, Mo.

"This monthly enables us to utilize at least several years more of life than it would be possible were we obliged to wait its publlcation in book-form at the hands of some compiler." - The Writing Teacher and Business Advertiser, New York.

Tine Popclar Science Movthly is published in a large octaro, handsomely printed on clear type, and, when the subjects admit, fully illustrated. Each number contains 128 pages.

\section{TERMS: $\$ 5$ per Anmum, or Fifty Cents per Number.}

Any person remitting $\$ 20.00$ for four yearly subscriptions, will receive an extra copy gratis, or five yearly subscriptions for $\$ 20.00$.

Now Realy, Vols. I., II., III., and IV.. of The Popular Science Mronthly, embracing the. Numbers froin 1 to 24 (Mas, 1s72, to April, 15i4). 4 vols., Svo. Cloth, $\$ 3.50$ per vol. Half Morocco, $\$ 6.50$ per vol.

For Sale, Binding Cases for Popular Science Monthly. Vols. I., II.. III.. and IT. These covers are prepared expressly for binding the volumes of Tile Popdlar Sciexce MoNTilly ss they appear, and will be sent to Subscribers on receipt of price. Any binder can attach the corers at trifling expense. Price, 50 cents each.

Agentz Wanted.

D. APPLETON \& CO., Publishers, $549 \& 551$ Broadway, New York. 


\section{APPLETONS' JOURNAL,}

\section{FOR 1875 .}

Appletoxs' Jocrsal will sustain, during the ensuing year, its reputation for general excellence. The publishers will endeavor, more strenuously than ever, to furnish a periodical of a high class, one which shall embrace a wide scope of topics, and afford the reader, in addition to an abundance of entertaining popular literature, a thorough survey of the progress of thouglit, the advance of the arts, and the doings in all branches of intellectual effort. As the design is to make a superior literary journal, engravings will be employed only when they serve to illustrate the text, and never merely as pictures.

Without adhering too rigidly to any set plan, the contents will be grouped approximately as follows:

I. Litreature of Romasce, consisting of popular serial novels, from both American and Finglish writers, and the best short stories obtainable, whether from native or foreign writers.

II. Travkz, Adventere, and Discovery, embracing papers descriptive of places, tales of adrenture and discovery, with notes of all that is doing in the way of exploration, or that is brought to light of new and unfamiliar countries.

III. Natere axd Natural IIsforv, under which will be given entertaining papers on the characteristics of the eartl's surface, the habits of animals, and all that pertains to the physical world around us.

IV. Sochal Themes, including papers expressing tho ideas of capable observers on social progress, the arts and felicities of the household, and such matters as pertain to our daily lives.

V. TnF ARTs, embracing criticisms of new paintings, new architecture, etc.; observations on decorative and household art, and a general survey of the progress of the arts in all their branehes.

VI. Mrscellivr will cover selections from new books, brief translations from Continental journals, and extracts giving the core of the more noteworthy papers in the English magazines.

VII. ScIExce AxD INvextion will consist of popular papers on subjects covered by these terms, and wlll record the progress made therein.

VIII. New Books will be carefully and impartially reviewed, in the sole inteiest of the reader; and notes will be subjoined, affordlng intelligence in regard to literary matters here and abroad.

IX. Ccrrent Topics will consist of the editor's glances at themes occupying the public mind; at various utterances by leading spirits in literature, philosophy, and critieism; and at the gayeties and amusements of society. It is designed to make this department notatle for its entertaining rivacity.

The broad purpose of the editors will be to make a magazine of weekly issue, that shall rival in interest and variety the regular monthly publications; and for this purpose the space at their command enables them to give much more material for the same yearly subscription than that contained in the largest of the monthly magazines.

\section{Published Weekly. Price 10 Cents per Number; or $\$ 4$ per Annum, in advance.}

By the recent Post-Office Lxw, the postage on all periodicals, after January 1, 1875, must be prepaid by the pub!ishers. Subscribers, therefore, will hereafter reccive their numbers without charges for postage.

Any person procuring Five Yearly Subscriptions, for weekly numbers, and remitting $\$ 20.00$, will be entitled to a copy for one year gratis.

In remitting hy mail, a post-office order or draft, payable to the order of D. ApPI.ETox \& Co., is preferable to bank-notes, as, if lost, the order or draft ean be recovered without loss to the sender.

Volumes begin with January and July of each year.

Appletons' Jocrxal and either Harper's Weekly, Harper's Bazar. Harper's Magazine, Lippincott's Magazine, the Atlantic Monthly, Scribner's Monthly, or the Galaxy, for one year, on receipt of 8.50, which includes prepayment of postage; Arpletoss' Jovrsal and Littell's Liring Age. for $\$ 10.50$, including postage; tho Jockxal and Poperar Screxce Mostnly, for \$s, including postage prepaid by the publishers.

For those who prefer it, the Jovrsar, is put np in Moxtmex PARTs, and in this form its scope and varioty, as compared with other magazines, beeme conspicuously apparent. Snbscription price, $\$ 4.50$ per annum, including postage prepaid by the publishers.

\section{APPIETOM \& CO., Publishers, New York.}




\section{Heat as a Mode of Motion.}

One vol., 12mo. Cioth, \$2.00.

"My arm nas been to rise to the leves of these ouestions rom a oasis so eiementary, that a person possessing any imaginative facuty and power of concentration might accompany me." - From Autnor's Freface.

\section{On Sound.}

A Course of Eignt Lectures delivered at the Royal Institution of Great Britain. One vol. With Illustrations. I2mo. Cloth, \$2.00.

"In the following pages I have tried to render the science of Acoustics interesting to all intelligent persons, including those who do not possess any special scientific culture."-From Author's Prefaee.

\section{Fragments of Science for Unscientific People.}

A Series of Detached Essays, Lectures, and Reviews. One vol., 12mo. Cloth, \$2.00.

" My motive in writing these papers was a desire to extend sympathy for science beyond the limits of the scientific public. - . From America the impulse came which induced me to gather these "Fragments,' and to my friends in the United.States I dedicate them."-From Author's Preface.

\section{Light and Electricity.}

Notes of Two Courses of Lectures before the Royal Institution of Great Britain. One vol., I2mo. Cloth, $\$ 1.25$.

"In thus clearly and sharply stating the fundamental principles of Electrical and Optical Science, Prof. Tyndall has earned the cordial thanks of all interested in education." -From American Editor's Preface.

\section{Hours of Exercise in the Alps.}

Orse vol., I2mo. With Illustrations. Cloth, \$2.00.

"The present volume is for the most part a record of bodily action, written partly to preserve to myself the memory of strong and joyous hours, and partly for the pleasure of those who find exhilaration in descriptions associated with mountain-life."-From Author's Preface.

\section{Faraday as a Discoverer.}

One vol., I2mo. Cloth, \$r.oo.

"It has been thought desirable to give you and the world some image of Michael Faraday as a scientific investigator and discoverer. . . . I have returned from my task with such results as I could gather, and also with the wish that these results were more worthy than they are of the greatness of my theme." The Author.

\section{Forms of Water, in Clouds, Rain, Rivers, Ice, and Glaciers.}

This is the first volume of the International Scientific Series, and is a valuable and interesting work. One vol., 12mo. Cloth, \$r.50.

Contributions to Molecular Physics in the Domain of Radiant Heat.

A Series of Memoirs published in the "Philosophical Transactions" and "Philosophical Magazine." With Additions. I vol., 8vo. Cloth, \$5.00.

Lectures on Light delivered in America. With numerous Illustrations. Small I2mo. Illustrated. Paper, 75c.; cloth, \$I. D. APPLETON \& C0., Publishers, 549 \& 551 Broadway, N. Y. 


\section{International Scientific Series.}

\section{NOW IEADY.}

No. 1. FORMS OF WATER, in Clouds, Rain, Rivers, Ice, and Glaclers. By Prof. Jons Trx DALl. LL. D., F.R.S. 1 vol. Clotb. Price, $\$ 1.50$.

No. 2. PHYSICS AND FOLITICS; or, Thoughts on the Application of the Principles of "Natural Selection" and "Inheritance" to Political Society. By Walter Bagenot, Esq. author of "The English Constitutlon." 1 vol. Cloth. Price, \$1.50.

No. 3. Foods. By Enward Smitu, M. D., LL. B., F. R. S. 1 rol. Cloth. Price, \$1.75.

No. 4. MIND AND BODY. The Theories of their Relation. By Alex. Bats, LL. D., Professor of Logic in the University of Aberdeen. 1 vol., $12 \mathrm{mo}$. Cloth. Price, $\$ 1.50$.

No. 5. THE STUDY OF SOCIOLOGY. By Herbert Spescer. 1 vol., 12mo. Cloth, \$1.50.

No. 6. THE LOCOMOTION OF ANIMALS, as exemplified in Walking, swimming, and Flying. By G. Bell Pettignew, M. D. 1 vol., 12mo. Cloth. Price, $\$ 1$. ī.

No. 7. THE NEW CHEMISTRY. By Prof. Josiau P. Cooke, Jr., of Harvard Unirersity. 1 vol., 12mo. Cloth. Price, $\$ 2.00$.

No. 8. ThE CONSERVATION OF ENERGY. By Prof. Balfour Stewart, La. D.. F. R. S. 1 vol., 12mo. Cloth. Prico, $\$ 1.50$.

No. 9. RESPONSIBILITY IN MENTAL DISEASE. By Dr. Hexry MatdsLer. 81.50.

No. 10. THE SCIENCE OF LAW. By Prof. Sneldon Avos. Price, \$1.75.

No. 11. THE ANIMAL MÅHINE. By Prof. E. J. Marex, of the College of France; meu ber of the Academy of Medicine. Price, $\$ 1.75$.

\section{PROSPECTUS.}

D. Appletox \& Co. have the pleasure of announcing that they have made arrangements for publishing, and have recently commenced the isstuo of a SERIEs of Popular Moxorisapis, or small works, under the above title, which will embody the results of recent incuiry in the most interesting departments of advancing science.

The eharacter and scope of this series wll be best indicated by a referenec to the names and subjects included in the subjoined list, from whieh it will be seen that the coijreration of the most distinguished professors in England, Germany, France, and the Tnited States, has been secured, and negotiations are pending for coutributions from other eminent sclen tific writers.

The works will bo issued simultaneously in New York, London, Paris, and Lcipsic.

The International. Scientifio Senies is entirely an Ameriean project, and was originated and organized by Dr. E. L. Youmans, who spent the greater part of a year in Europe, arranging vith authors and publishers.

The forthcoming volumes are as follows:

Prof. T. H. HuxLex, LL. D., F. R. S., Bodily Motion and Consciousuess.

Sir Joнr Luввоск, Bart., F. R. S., The Antiquity of $M a n$.

Prof. Rubolipn Vircnow (of the University of Berlin), Morbid Plhysiological Action.

Dr. H. Cilartion Bastian, M. D., F. R. S., The Brain as an Organ of Mind.

Prof. W. Tulstleton Dyer, B. A., B. Sc., Form and Irabit of Flowering Plunts.

Prof. W. Kingdos Clifrurd, II. $\Lambda_{\text {, }}$ The First Principles of the Exact Sciences explained to the Non-Mathematical.

Mr. J. N. Lockyer, F. R.S., Spectrum Analyxis.

W. Lauder Lindsay, M. D., F. R. S. E., Mind in the Lower Animals.

Prof. Jayes D. Daxa, M. A., LL. D., On Cephalization; or. IIead Domination in its Relation to Struclure, Grade, and Derelopment.

Prof. S. W. Jomssos, M. A., On the Nutrition of Plants.

Prof. Atstin Fuivt. Jr., M. D., The Nerrous System, and its Relation to the Bodily Functions.

Prof. W. D. Wminex, Modern Linguistic Science.

Prof. A. C. Ransar, LL. D., F. R. S., Eurth Sculpture.

Prof. Lacaze-Dutmers, Zoology since Cuvier.
Dr. IIenry Matdsley, Merponsibility in Disease.

Pruf. Michafr Foster, M. D., Protoplasm and the Cell Theory.

liev. M. J. Berkelex. M. A., F. I. S., Fungi; their Nature. Influences, and Cres.

Prof. (lacde Bersarn (of the College of France), Plysical and Metiphysical Phenomena of Life.

Prof. A. Quetelet (of the Brussels A calemy of Seiences). Social Physics.

Prof. A. De Quatrefages, The Negro Races.

I'rof. C. A. Yousg, Ph. D). (of Jartmonth College), The sun.

Prof. Braxsteix (Tnirersity of Ilalle), The Physiolog!y of the senres.

Prof. IIERiax (University of Zurich), On Respiration.

Prof. Lecchard (Tniversity of I.elpsic), Outlines of Chemical Organizution.

Prof. Rees (Ćniversity of Erlangen), On Parasitic Plants.

Prof. Voite (Polvtechnlc Academy, Berlin), The Chemical Effects of Light.

Prof. WUNuT (University of Strasbourg), On sound.

Prof. Scuvint (University of Strasbourg), The Theory of Descent-Duranism.

Prof. Rosextiual (University of Erlangen), Physiology of $\mathbf{H}$ uscles and Nerces.

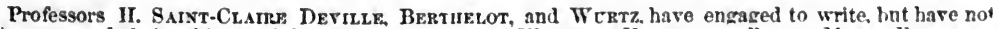
get announced their subjects. Other eminent althors, as W ALACE, HeLvHOLTz, PARKs, MILXE-EDWARDS

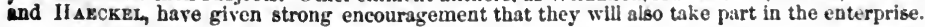

D. APPLETON \& C0, Publishers, 549 \& 551 Broadway, N. Y 


\section{Recents Scientifíc Pnblications by D. Appleton \& Co.}

IYELL.-PRINCIPLES OF GEOLOGY (Vol. I.); or, the Modern Changes of the Earth and its Inhabitants considered, as illustrative of Geology. By Sir CHanLes LyeiL,

Bart., M. A., F. R. S. In two volumes. 8 vo.

This is a new edition (the eleventh) of this great work. It has been entirely and carefully revised, and is illustrated with uumerous Maps, Plates, and Woodcuts. Vol.II. will be issued during
the present year.

SCHELLEN.-SPECTRUM ANALYSIS, in its Application to Terrestrial Substances, and the Physical Constitution of the Heavenly Bodies. Familiarly explained by Dr. H. Schellen, Director der Realschule I. O. Cologne, Ritter des Rothen Adlerordens IV. KL., Associate of several Learned Societies. Translated from the second enlarged and revised German edition, by Jane and Caroline Lassell. Edited, with Notes, by Wm. Huggins, LL. D., D. C. L., F. R. S. With numerous Woodcuts, Colored Plates, and Portraits ; also, Angström's and Kirchhoff's Maps. 455 pp. 8vo. Cloth. Price, \$6.

The colored plates illustrating this edition of the work, requiring great care in printing, were executed in London.

"This admirable work docs credit to, or shonld we say is worthy of, the author, the translators, and the editor. The first part treats on the artificial sources of high degrecs of heat and light; the second on Spectrum Analysis in its application to the heavenly bodies. We must approve the method followed in the translation, and by the editor. In many translations the view's of the author are suppressed, in order that the views of the translator or editor may be expounded; but here Dr. Huggins, however leniently such a fault night have been lcokcd upon with him, has permitted the author's views to remain intact, clearly stating his own, and wherein lies the difference."-Chemical News,

LUBBOCK.-PRE-HISTORIC TIMES, as illustrated by Ancient Remains, and the Manners and Customs of Modern Savages. By Sir John LubBock, Bart., M. P., VicePresident of the Royal Society; of the British Association, etc. 1 vol., 8ro, $640 \mathrm{pp}$.

Plates and Illustrations. Cloth. Price, $\$ 5.00$.

"Fully satisfied that religion and science cannot in reality be at variance, I have striven, in the present publication, to follow ont. the rule laid down by the Bishop of London in his excel. lent lecture, delivered last year at Edinburgh. 'The man of Ecience' eays Dr. Tait. 'ought to go on honestly, patiently, diffidently, observing and stirring up his observations, ard carrying his reasonings unflinchingly to their legitimate conclusions, convinced that it would be treason to the majesty at once of science and of religion, if he sought to help either by swerving ever so little from the straight rule of truth." "-Extract from Freface.

HUXLEY.-ANATOMY OF VERTEBRATED ANIMALS. By Thomas Henry HexLEY, LL. D., F. R. S., author of "Man's Place in Nature," "On the Origin of Species," "Lay Sermons and Addresses," etc. 1 vol., 12mo. Cloth. Price, \$2.50.

The former works of Professor Huxley leave no room for doubt as to the importance and value of his new volume. It is one which will be very acceptable to all who are interested in the subject of which it treats.

"This long-expected work will be cordially welcomed by all students and teachere of Comparative Anatomy as a compendious, reliable, and, notwithstanding its small dimensions, most comprehensive guide on the subject of which it treats. To praise or to criticise the work of so accomplished a master of his favorite science would be equally out of place. It is enough to say that it realizes, in a remarkable degree, the anticipations which have been formed of it; and that it presents an extraordinary combination of wide, general views, with the clear, accurate, and succinct statement of a prodigious number of individual facts."-Nature.

NICHOLSON.--A MANUAL OF ZOOLOGY, for the Use of Students. With a General Introduction on the Principles of Zoology. By Hexry Alleyne Nicholson, M. D. D. Sc., M. A., Ph. D. (Gött.), F. R. S. E., F. G. S., Professor of Natural History and Botany in University College, Toronto; formerly Lecturer on Natural History in the Medical School of Edinburgh; Vice-President of the Geological Society of Edinburgh. Second edition, revised and considerably enlarged. 1 vol., $8 \mathrm{vo}, 673$ pages.

DESCHAFEL-ELEMENTARY TREATISE ON NATURAL PHILOSOPHY. By A. Privat Deschanel, formerly Professor of Plyssics in the Lycée Louis-le-Grand, Inspector of the Academy of Paris. Translated and edited, with extensive additions, by J. D. Everetr, Professor of Natural Philosophy in the Queen's College, Belfast. To be completed in Four Parts. Part I. Mechanics, Hydrostatics, and Pneumatics; Part II. II eat, and Part III. Physics, are now ready in separate volumes. Copiously illustrated. 8vo. Flexible cloth. Price, $\$ 2.00$ each.

HINTON.-MAN AND HIS DWELLING-PLACE. By JANES HINTON, author of the "Mystery of Pain." 1 vol., 12mo. Cloth. Price, \$1.75.

The author of this work holds a unique position among the thinkers of the age. He brings to the discussion of man and Nature, and the bigher problems of human life, the latest and most thornugh scientific preparation, and constantly employs the later dynamic philosophy in dealing with them. But he is broader than the scientific school which he recognizes, but with him the moral and reiigions elements of man are supreme. He conjoins strict science with high spiritu. ality of view. "Man and his Dwelling-Place" is here rewrittcn and compressed, and presents, in a pointed and attractive style, original aspects of the most engaging questions of the time. 


\section{S'cientific Pablications of D. Appleton \& C'.}

DARWIN.-THE ORIGIN OF SPECIES

by Means of Natural Selection, or the Preservation of Favored Races in the Struggle for Life. New and revised edition, with Additions. With Copious Index. 1 rol., $12 \mathrm{mo}$. Cloth. Price, $\$ 2.00$.

THE DESCENT OF MAN, and Selection in Relation to Sex. With many Illustrations. 2 vols., $12 \mathrm{mo.}$ Cloth. Price, $\$ 4.00$.

This work, in which the author expresses his pecnliar views boldly and forcibly, has been widely read and criticised, and, whatever may be the opinion arrived at in regara to his theories, the reader cannot fail to obtain pleasure and profit in its perusal.

JOURNAL OF RESCARCHES into the Natural History and Geology of the Countries visited cuning the Voyage of II. M. S. Beagle rounc the World. A new edition, uniform with "Origin If Species" and "Descent of Man. 1 vol., 12mo. Cloth. Price, $\$ 2.00$.

An interesting account of a voyage of scientific research and discovery.

HUXLEY.-MAN'S PLACE IN NATURE. 1 vol., $12 \mathrm{mo}$. Cloth. Price, $\$ 1.25$.

ON THE ORIGIN OF SPECIES 1 vol., $12 \mathrm{mo}$. Cloth. Price, $\$ 1.00$.

LAY SERMONS, Addresses, and Reviews. 1 vol., 12mo. Cloth. Price, $\$ 1.75$.

ELEMENTS OF PHYSIOLOGY, and Hygiene. By T. H. Huxley and W. J. Youmans. 1 vol, $12 \mathrm{mo}$. $\$ 1.75$.

MORE CRITICISMS ON DARIVIN, and Administrative Nihilism. 1 vol., $12 \mathrm{mo}$. Price, 50 cents.

Valuable to all who are interested in scientific study or reading.

PROCTOR (R. A.)-OTHER WORLDS THAN OURS : the Plurality of Worlds, Studied under the Light of Recent Scientific Researches. By Richard $A$. Proctor, B. A., F. R. A. S., author of "Saturn and its System," "Sun Views of the Earth," "Half Hours with the Telescope," etc. With Illustraiions, some colored. 12mo. Cloth. Price, $\$ 2.50$.
PROCTOR (R. A.) - LIGHT SCIENCE FOR LEISURE HOURS. A Scries of Familiar Essays on Seientific Subjects, Natural Phenomena, ctc. 1 vol., 12 mo. Cloth. Price, $\$ 1.75$.

An instructive and entertaining rolume to the student of science, and of astrouomy.

SPENCER (HERBERT). - E D U C A . TION, Intellectual, Moral, and Physi. cal. 12mo. Cloth. Price, $\$ 1.25$.

FIRST PRINCIPLES. 12mo. Cloth. I.: 2 c, $\$ 2.50$.

CLASSIFICATION OF THE SCIENCES. 8vo. Paper. Price, 25 cts.

EsSAYS : Moral, Political, and Æs. thetic. I vol., 12mo. Cloth. Price, $\$ 2.50$.

ILLUSTRATIONS OF UNIVERSAL PROGRESS. A Selection of his best Papers. 1 vol., 12 mo. C'oth. Price, \$2.50.

SOCIAL STATICS; or, the Conditions Essential to Human Happiness Specified, and the First of them Devel. oped. 1 vol., 12mo. Cloth. Price, $\$ 2.50$.

THE PRINCIPLES OF BIOLOGY,

2 vols., 12mo. Cloth. Priec, $\$ 5.00$.

THE PRINCIPLES OF PSYCHOL.

OGY: Part I. Data of Psychology. 1 vol. Paper. Price, 75 cents; Part II. The Inductions of Psychology. 1 vol., 8vo. Paper. Price, 75 cents; Part III. General Synthesis ; Part IV. Special Synthesis. Puper. Price, $\$ 1.00$; Part V. Physical Synthesis. Paper. Price, 75 cents.

THE PRINCIPLES OF PSYCHOL.

OGY. Yol. I., 8vo. Cloth. Price, \$2.50.

WORKS. 7 rols., 8ro. Half Calf.

Price, $\$ 28.00$.

The works of Herbert Spencer have attained a prominence and a reputation in America even greater than in England, and he stands tho acknowledged leader of a sehool of philosophy which connts many students and thinkers among his followers. 


\section{Scientific Pnblications of D. Appleton \& Co.}

TYNDALL-HEAT AS A MODE OF MOTION. 1 vol, $12 \mathrm{mo}$. Cloth. Price, $\$ 2.00$.

ON SOUND : A Course of Eight Lectures delivered at the Royal Institution of Great Britain. By John Tyndall, LL. D., F. R. S. Illustrated. 1 vol., 12 mo. Cloth. Price, $\$ 2.00$.

FRAGMENTS OF SCIENCE FOR UNSCIENTIFIC PEOPLE. A Series of Detached Essays, Lectures, and Reviews. $12 \mathrm{mo}$. Cloth. Price, $\$ 2.00$.

- LIGHT AND ELECTRICITY : Notes of Two Courses of Lectures before the Royal Institution of Great Britain. 12mo. Cloth. Price, \$1.25.

HOURS OF EXERCISE IN THE ALPS. With Illustrations. $12 \mathrm{mo}$. Cloth. Price, $\$ 2.00$.

ON RADIATION. The "Rede" Lecture delivered in the Senate House before the University of Cambridge, England, on Tuesday, May 16, 1865. Author of "Heat considered as a Mode of Motion." 1 vol., 12mo. Cloth. Price, 50 cents.

Important works by one of England's greatest scientists.

YOUMANS (E. L.)-CLASSS-BOOK OF CHEMISTRY. New edition. $12 \mathrm{mo}$. Price; \$1.75.

ATLAS OF CHEMISTRY. 1 vol., 4to. Cloth. Price, $\$ 3.00$.

- CHART OF CHEMISTRY, on Roll.

er. Price, $\$ 8.00$.

HAND-BOOK OF HOUSEHOLD SCIENCE. $12 \mathrm{mo}$. Price, $\$ 1.75$. THE CULTURE DEMANDED BY MODERN LIFE : A Series of Address. es and Arguments on the Claims of Scientific Education. Edited, with an Introduction on Mental Discipline in Education, by Edward L. Youmans, M. D. 1 vol., $12 \mathrm{mo}$. Cloth. Price, $\$ 2.00$.

CORRELATION AND CONSER. VATION OF FORCES : A Series of Expositions by Prof. Grove, Prof. Helmholtz, Dr. Mayer, Dr. Faraday, Prof. Liebig, and Dr. Carpenter. Edited, with an Introduction and Brief Biographical Notices of the Chief Promoters of the New Views, by Edward $\mathbf{L}$. Youmans, M. D. 1 vol., 12mo. Cloth. Price, $\$ 2.00$.

The Atiantic Monthly says of Professor Youmuns: "His time seems to be wholly devoted to the disinterested service of science and of his fellow-men."

\section{Appleton \& Co. have just published: HOW THE WORLD WAS PEOPLED,}

A TREATISE ON ETHNOLUGY.

By the Rev. EDWATE FONTAINE,

PROFTSSOR OF THEOLOGY AND NATURAL SCIENCE; A MEMBER OF THE NEW YORK HistoricAL SOCIETY, AND OF THE ACADEMIES OF SCIENCES OF NEW ORLEANS, BALTMORE, ETC.

This learned, but simple and intelligible, book is the result of more than thirty years' careful study of ancient and modern history, and the archæology of all nations. By his independent researches in unexplored fields, aided by a thorough knowledge of geology, physical geography, and natural history, as far as those sciences have been matured by others, he has succeeded, he thinks, in so arranging incontrovertible facts as to settle satisfactorily the disputed question of the origin and antiquity of mankind. His researches have brought him to the conclusion of Alexander von Iumboldt, and his brother William, that we are all the descendants of one originally created pair, as the Bible teaches. This places him in opposition to Sir Roderick Murchison, Professor Agassiz, Mr. Darwin, and many other eminent naturalists. Mr. Fontaine's book will commend itself especially to students of theology, clergymen of all churches, and the professors of colleges. Every argument heretofore advanced against the biblical account of the origin of man is fairly stated, and even more strongly propounded than by its advocates. It is then replied to candidly and clearly, and, as the anthor thinks, conclusively. He seems to believe that no comprehensive and honest reasoner can read the book carcfully without coming to the conclnsion that the Mosaic cosmogony is correct, and that the account given in Genesis of the origin of manlind is true. 





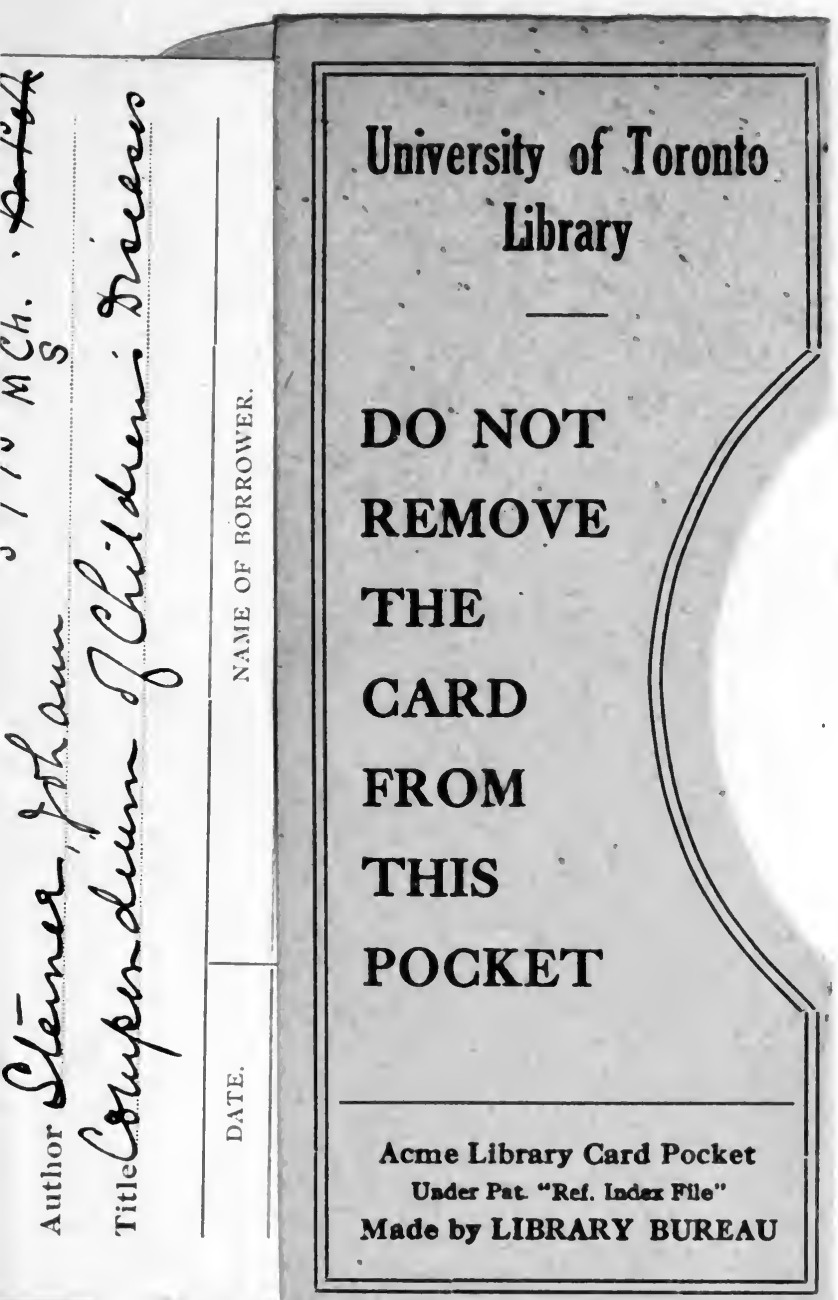


$\therefore$

$\therefore$
$\therefore$
$\therefore \quad \therefore \quad$
$\therefore$
4

$4=$

$\therefore \quad 0$ 